\title{
Asymptotic Behaviour of Variation of Pure Polarized TERP Structure
}

\author{
by
}

Takuro Mochizuki

\begin{abstract}
The purpose of this paper is twofold. One is to give a survey of our study on reductions of harmonic bundles, and the other is to explain a simple application in the study of TERP structures. In particular, we investigate the asymptotic behaviour of the "new supersymmetric index" for variation of pure polarized TERP structure.
\end{abstract}

2010 Mathematics Subject Classification: 32L05, 14D07.

Keywords: harmonic bundle, TERP structure, new supersymmetric index.

\section{$\S 1$. Introduction}

In our previous papers [21, 22] and 23], we studied asymptotic behaviour of tame and wild harmonic bundles. Briefly, one of the main results is the following sequence of reductions of harmonic bundles:

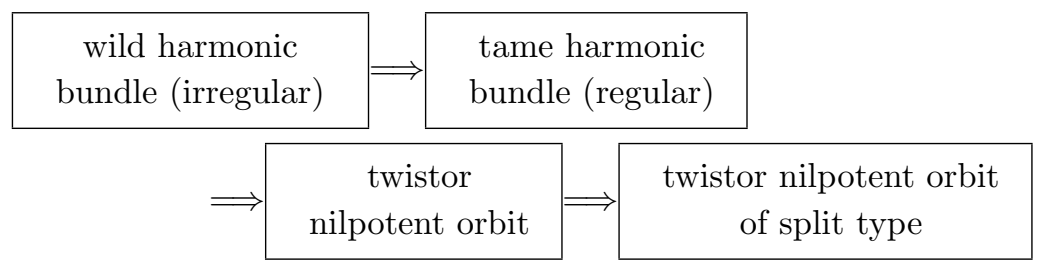

Although a reduced object is simpler than the original one, it still gives a good approximation. Moreover, a twistor nilpotent orbit of split type comes from a variation of polarized pure Hodge structure, whose asymptotic behaviour was deeply studied by E. Cattani, A. Kaplan, M. Kashiwara, T. Kawai and W. Schmid. Thus,

This is a contribution to the special issue "The golden jubilee of algebraic analysis".

Communicated by M. Kashiwara. Received December 2, 2009. Revised April 20, 2010.

T. Mochizuki: RIMS, Kyoto University, Kyoto 606-8502, Japan;

e-mail: takuro@kurims.kyoto-u.ac.jp

(C) 2011 Research Institute for Mathematical Sciences, Kyoto University. All rights reserved. 
we can say that the asymptotic behaviour of wild harmonic bundles is pretty well understood.

The main purpose of this paper is twofold. One is to give a survey of these reductions, and the other is to explain a simple application in the study of TERP structures.

C. Hertling 10 initiated the study of TERP structures inspired by mathematical physics and singularity theory. The study was further developed by Hertling and C. Sevenheck. For example, they investigated the "nilpotent orbit" [12], asymptotic behaviour of tame variation of TERP structure and classifying spaces [13]. We refer to the above papers and a survey [14] for more details and specifications.

Remark 1. The "nilpotent orbit" of [12] is called "HS-orbit" (Hertling-Sevenheck orbit) in this paper. A nilpotent orbit is a very special kind of variation of polarized Hodge structure, which plays an important role in Hodge theory. We can consider several kinds of generalization in the theory of TERP structures and twistor structures. One is the HS-orbit. Another one is the twistor nilpotent orbit studied in 22], which we will mainly use in this paper.

Remark 2. We prefer to regard a TERP structure as an integrable twistor structure with a real structure and a pairing studied by C. Sabbah. It is called a twistor-TERP structure in this paper.

We will give an enrichment of the sequence 1.1 with TERP structures or integrable twistor structures. As an application, we will study the behaviour of the "new supersymmetric index" of variation of pure polarized TERP structure. Let $\nabla$ be a meromorphic connection on $V=\mathcal{O}_{\mathbb{P}^{1}}^{\oplus r}$ admitting a pole at $\{0, \infty\}$ of order at most two. Let $d$ be the natural connection on $V$. Then we have the expression $\nabla=d+\left(\lambda^{-1} \mathcal{U}_{1}-\mathcal{Q}-\lambda \mathcal{U}_{2}\right) d \lambda / \lambda$, where $\mathcal{U}_{i}, \mathcal{Q} \in \operatorname{End}(V)$. If $(V, \nabla)$ is equipped with a real structure and a polarization (see Subsection 2.1.5), there is some more restriction on them. Anyway, $\mathcal{Q}$ is called the new supersymmetric index of $(V, \nabla)$. We set $X:=\left\{\left(z_{1}, \ldots, z_{n}\right)|| z_{i} \mid<1\right\}$ and $D:=\bigcup_{i=1}^{n}\left\{z_{i}=0\right\}$. Let $\left(\mathcal{V}, \widetilde{\mathbb{D}}^{\Delta}, \mathcal{S}, \kappa\right)$ be a variation of pure polarized twistor-TERP structure of weight 0 on $\mathbb{P}^{1} \times(X-D)$. (See Subsection 2.1.) It is called unramifiedly good wild (resp. tame) if so is the underlying harmonic bundle $\left(E, \bar{\partial}_{E}, \theta, h\right)$. (See Subsection 6.1.) For each point $P \in$ $X-D$, we have the new supersymmetric index $\mathcal{Q}_{P} \in \operatorname{End}\left(\mathcal{V}_{\mid \mathbb{P}^{1} \times P}\right) \simeq \operatorname{End}\left(E_{\mid P}\right)$ of $\left(\mathcal{V}^{\triangle}, \widetilde{\mathbb{D}}^{\triangle}\right)_{\mid \mathbb{P}^{1} \times P}$, and thus we obtain a $C^{\infty}$-section $\mathcal{Q}$ of $\operatorname{End}(E)$. We are interested in the behaviour of $\mathcal{Q}$ around $(0, \ldots, 0)$. We will show the following:

- In the case of a twistor-TERP nilpotent orbit of split type, the new supersymmetric index can be easily computed from the data of the corresponding polar- 
ized mixed twistor-TERP structure. In particular, the eigenvalues are constant. (See Section 3)

- From a twistor-TERP nilpotent orbit $\left(\mathcal{V}, \widetilde{\mathbb{D}}^{\triangle}, \mathcal{S}, \kappa\right)$, we obtain a twistor-TERP nilpotent orbit of split type $\left(\mathcal{V}_{0}, \widetilde{\mathbb{D}}_{0}^{\triangle}, \mathcal{S}_{0}, \kappa_{0}\right)$, by taking Gr (graduation) with respect to the weight filtration. (Precisely, Gr is taken for the corresponding polarized mixed twistor-TERP structure.) The new supersymmetric index $\mathcal{Q}$ of $\left(\mathcal{V}, \widetilde{\mathbb{D}}^{\Delta}\right)$ can be approximated by the new supersymmetric index $\mathcal{Q}_{0}$ of $\left(\mathcal{V}_{0}, \widetilde{\mathbb{D}}_{0}^{\Delta}\right)$ up to $O\left(\sum\left(-\log \left|z_{i}\right|\right)^{-1 / 2}\right)$. In particular, the eigenvalues of $\mathcal{Q}$ are constant up to $O\left(\sum\left(-\log \left|z_{i}\right|\right)^{-\delta}\right)$ for some $\delta>0$. (See Section 4 $)$

- A tame variation of polarized pure twistor-TERP-structure $\left(\mathcal{V}, \widetilde{\mathbb{D}}^{\triangle}, \mathcal{S}, \kappa\right)$ is reduced to a twistor-TERP nilpotent orbit $\left(\mathcal{V}_{0}, \widetilde{\mathbb{D}}_{0}^{\triangle}, \mathcal{S}_{0}, \kappa_{0}\right)$. It is associated to the limit mixed twistor-TERP structure which was essentially considered in [13] as an enrichment of the limit mixed twistor structure of 22. We can approximate the new supersymmetric index $\mathcal{Q}$ of $\left(\mathcal{V}, \widetilde{\mathbb{D}}^{\triangle}\right)$ by the new supersymmetric index $\mathcal{Q}_{0}$ of $\left(\mathcal{V}_{0}, \widetilde{\mathbb{D}}_{0}^{\Delta}\right)$ up to $O\left(\sum\left|z_{i}\right|^{\epsilon}\right)$ for some $\epsilon>0$. In particular, the eigenvalues of $\mathcal{Q}_{0}$ approximate those of $\mathcal{Q}$ up to $O\left(\sum\left|z_{i}\right|^{\epsilon^{\prime}}\right.$ ) for some $\epsilon^{\prime}>0$. (See Subsection 7.4 for more precise statements.)

- A wild variation of polarized pure twistor-TERP structure $\left(\mathcal{V}, \widetilde{\mathbb{D}}^{\Delta}, \mathcal{S}, \kappa\right)$ is reduced to a tame one $\left(\mathcal{V}_{0}, \widetilde{\mathbb{D}}_{0}^{\Delta}, \mathcal{S}_{0}, \kappa_{0}\right)$, which is Gr with respect to Stokes filtrations. We can approximate the new supersymmetric index $\mathcal{Q}$ of $\left(\mathcal{V}, \widetilde{\mathbb{D}}^{\triangle}\right)$ by the new supersymmetric index $\mathcal{Q}_{0}$ of $\left(\mathcal{V}_{0}, \widetilde{\mathbb{D}}_{0}^{\triangle}\right)$ up to a term with exponential decay. In particular, the eigenvalues of $\mathcal{Q}_{0}$ approximate those of $\mathcal{Q}$ up to exponential decay. (See Subsection 7.3 for more precise statements.)

In each case, we will construct a $C^{\infty}$-map $\mathcal{V}_{0} \rightarrow \mathcal{V}$, which does not preserve but approximates the additional structures. (More precisely, $\mathcal{V}_{0}$ should be twisted.) It would be interesting to clarify the precise relation between these results and the celebrated nilpotent orbit theorem for Hodge structures due to W. Schmid [28]. (See also [13].)

As a corollary, we obtain the convergence of the eigenvalues of new supersymmetric indices of wild harmonic bundles on a punctured disc. In his recent work (Section 3 of 26]), Sabbah studied the eigenvalues of new supersymmetric indices for polarized wild pure integrable twistor $D$-modules on curves. Since wild harmonic bundles are prolonged to polarized wild pure twistor $D$-modules [23, we can also deduce the above convergence in the curve case from his results.

We also show that if a TERP-structure induces an HS-orbit, then it is a mixed-TERP structure in the sense of [12] by using the reduction from wild to tame, which was conjectured by Hertling and Sevenheck. 
Outline of this paper. This paper is roughly divided into three parts. In Part I (Sections 2 4), we give some preliminaries and establish the equivalence between twistor nilpotent orbit and polarized mixed twistor structure. Part II (Sections 5 6] is a review of some results in [22] and [23, including the sequence [1.1]. In Part III (Sections 7 8), we study the enrichment of (1.1), the approximations, and their applications.

In Subsection 2.1 we recall integrable pure twistor structures and TERP structures and their variations in a convenient way; these were originally studied by Hertling, Sabbah and Sevenheck. We look at some basic examples in Subsection 2.2. In particular, we introduce the notions of integrable twistor nilpotent orbit and twistor-TERP nilpotent orbit. In Subsection 2.3, we deduce a convergence of integrable pure twistor structures and new supersymmetric indices. The result will be used several times. In Subsection 2.4 we consider a variation of polarized mixed twistor structure. In Subsection 2.4.2, we explain the reduction from a polarized mixed twistor structure to a polarized mixed twistor structure of split type. In Subsection 2.4.3, we give a $C^{\infty}$-splitting of weight filtrations compatible with nilpotent maps, which is a preparation for Section 4

In Section 3, we study polarized mixed twistor structures of split type with some additional structures. It is quite easy to handle. In Section 4 , we show the correspondence between twistor nilpotent orbits and polarized mixed twistor structures. In [22, a polarized mixed twistor structure was associated to a twistor nilpotent orbit. The converse was also established in the curve case. The higher dimensional case is new. The correspondence is easily enriched with integrability and real structures. We also show that a twistor nilpotent orbit is approximated by a twistor nilpotent orbit of split type.

In Section 5 , we give a review of Stokes structure and reductions for a family of meromorphic $\lambda$-flat bundles, studied in Sections 2 and 3 in 23 . We give some minor complementary results on connections along the $\lambda$-direction and pseudogood lattices.

In Section 6, we explain the reduction from unramifiedly good wild harmonic bundles to polarized mixed twistor structures, studied in [22] and [23]. We give a review on the prolongation of harmonic bundles in Subsection 6.3. Then, in Subsection 6.4. we review the reduction from unramifiedly good wild harmonic bundles to tame harmonic bundles as the Gr with respect to Stokes filtrations, which is one of the main results in 23 . In Subsection 6.5, we review the reduction from tame harmonic bundles to polarized mixed twistor structures as the Gr with respect to KMS-structure, which is one of the main results in [22]. Together with the result in Section 4 , we can regard it as the reduction to nilpotent orbits. 
In Section 7, we establish an enrichment of the reductions with integrability and real structure. One of the main issues is to obtain a meromorphic extension of the connection along the $\lambda$-direction. For that purpose, we prepare some estimate in Subsection 7.1. Then, it is easy to obtain the meromorphic prolongment of variations of integrable twistor structure and the enrichment of the sequence of reductions as in 1.1. We also show that the reduced one gives a good approximation of the original one. In particular, we obtain results on approximation of the new supersymmetric indices of wild or tame variation of integrable twistor structure.

In Section 8, we study the reduction of HS-orbit.

\section{$\S 2$. Preliminaries}

\section{$\S 2.1$. Integrable twistor structure}

We recall the notion of integrable twistor structures and TERP structures in a way convenient for us. See [10], [12] and [25] for the original definitions and for more details. We also recall twistor structures introduced in [32]. See also [21] and [22].

2.1.1. Some sheaves and differential operators on $\mathbb{P}^{1} \times X$. Let $\mathbb{P}^{1}$ denote the one-dimensional complex projective space. We regard it as the gluing of two complex lines $\mathbb{C}_{\lambda}$ and $\mathbb{C}_{\mu}$ by $\lambda=\mu^{-1}$. We set $\mathbb{C}_{\lambda}^{*}:=\mathbb{C}_{\lambda}-\{0\}$.

Let $X$ be a complex manifold. We set $\mathcal{X}:=\mathbb{C}_{\lambda} \times X$ and $\mathcal{X}^{0}:=\{0\} \times X$. Let $\widetilde{\Omega}_{\mathcal{X}}^{1,0}$ be the $C^{\infty}$-bundle associated to $\Omega_{\mathcal{X}}^{1,0}\left(\log \mathcal{X}^{0}\right) \otimes \mathcal{O}_{\mathcal{X}}\left(\mathcal{X}^{0}\right)$. We put $\widetilde{\Omega}_{\mathcal{X}}^{0,1}:=\Omega_{\mathcal{X}}^{0,1}$, and we define

$$
\widetilde{\Omega}_{\mathcal{X}}^{1}:=\widetilde{\Omega}_{\mathcal{X}}^{1,0} \oplus \widetilde{\Omega}_{\mathcal{X}}^{0,1}, \quad \widetilde{\Omega}_{\mathcal{X}}^{\bullet}:=\Lambda^{\bullet} \widetilde{\Omega}_{\mathcal{X}}^{1} .
$$

The associated sheaves of $C^{\infty}$-sections are denoted by the same symbols. Let $\widetilde{\mathbb{D}}_{X}^{f}$ : $\widetilde{\Omega}_{\mathcal{X}}^{\bullet} \rightarrow \widetilde{\Omega}_{\mathcal{X}}^{\bullet+1}$ denote the differential operator induced by the exterior differential $d$.

Let $X^{\dagger}$ denote the conjugate of $X$. We set $\mathcal{X}^{\dagger}:=\mathbb{C}_{\mu} \times X^{\dagger}$. By the same procedure, we obtain the $C^{\infty}$-bundles $\widetilde{\Omega}_{\mathcal{X}^{\dagger}}^{\bullet}$ with the differential operator $\widetilde{\mathbb{D}}_{X}^{\dagger f}$.

Their restrictions to $\mathbb{C}_{\lambda}^{*} \times X=\mathbb{C}_{\mu}^{*} \times X^{\dagger}$ are naturally isomorphic:

$$
\left(\widetilde{\Omega}_{\mathcal{X}}^{\bullet}, \widetilde{\mathbb{D}}_{X}^{f}\right)_{\mid \mathbb{C}_{\lambda}^{*} \times X}=\left(\Omega_{\mathbb{C}_{\lambda}^{*} \times X}, d\right)=\left(\widetilde{\Omega}_{\mathcal{X}^{\dagger}}^{\bullet}, \widetilde{\mathbb{D}}_{X}^{\dagger f}\right)_{\mid \mathbb{C}_{\mu}^{*} \times X^{\dagger}} \cdot
$$

By gluing them, we obtain the $C^{\infty}$-bundles $\widetilde{\Omega}_{\mathbb{P}^{1} \times X}^{\bullet}$ with a differential operator $\widetilde{\mathbb{D}}_{X}^{\triangle}$. Remark $3 . \widetilde{\mathbb{D}}_{X}^{f}$ and $\widetilde{\mathbb{D}}_{X}^{\dagger f}$ are also denoted by $d$, if there is no risk of confusion.

We have the decomposition $\widetilde{\Omega}_{\mathbb{P}^{1} \times X}^{1}=\xi \Omega_{X}^{1} \oplus \widetilde{\Omega}_{\mathbb{P}^{1}}^{1}$ into the $X$-direction and the $\mathbb{P}^{1}$-direction. The restriction of $\widetilde{\mathbb{D}}_{X}^{\triangle}$ to the $X$-direction is denoted by $\mathbb{D}_{X}^{\triangle}$. The restriction to the $\mathbb{P}^{1}$-direction is denoted by $d_{\mathbb{P}^{1}}$. By definition, the $(1,0)$-part of 
a section of $\widetilde{\Omega}_{\mathbb{P} 1}^{1}$ is logarithmic along $\{0, \infty\}$ with a pole of order 1 along $\{0, \infty\}$, at most, and the $(0,1)$-part is just a $C^{\infty}$-section of $\pi^{*} \Omega_{\mathbb{P 1}}^{0,1}$. Hence, we have the decomposition

$$
\widetilde{\Omega}_{\mathbb{P}^{1}}^{1}=\pi^{*} \Omega_{\mathbb{P}^{1}}^{1,0}(2\{0, \infty\}) \oplus \pi^{*} \Omega_{\mathbb{P}^{1}}^{0,1},
$$

into the (1,0)-part and the (0,1)-part, where $\pi$ denotes the projection $\mathbb{P}^{1} \times X \rightarrow \mathbb{P}^{1}$. We have the corresponding decomposition $d_{\mathbb{P}^{1}}=\partial_{\mathbb{P}^{1}}+\bar{\partial}_{\mathbb{P}^{1}}$.

Let $\nu: \mathbb{P}^{1} \rightarrow \mathbb{P}^{1}$ be a diffeomorphism. Assume $\nu$ satisfies one of the following: (A1) $\nu$ is holomorphic with $\nu(0)=0$ and $\nu(\infty)=\infty$.

(A2) $\nu$ is anti-holomorphic with $\nu(0)=\infty$ and $\nu(\infty)=0$.

In particular, we will often use the maps $\sigma, \gamma$ and $j$ :

$$
\sigma\left(\left[z_{0}: z_{1}\right]\right)=\left[-\bar{z}_{1}: \bar{z}_{0}\right], \quad \gamma\left(\left[z_{0}: z_{1}\right]\right)=\left[\bar{z}_{1}: \bar{z}_{0}\right], \quad j\left(\left[z_{0}: z_{1}\right]\right)=\left[-z_{0}: z_{1}\right] .
$$

The induced diffeomorphism $\mathbb{P}^{1} \times X \rightarrow \mathbb{P}^{1} \times X$ is also denoted by $\nu$. In the case (A1), we have the natural isomorphism $\Phi_{\nu}: \nu^{*} \widetilde{\Omega}_{\mathbb{P}^{1} \times X}^{\bullet} \simeq \widetilde{\Omega}_{\mathbb{P}^{1} \times X}^{\bullet}$ of $C^{\infty}$-vector bundles given by the ordinary pull back. In the case (A2), multiplication of $C^{\infty}$ functions on $\nu^{*} \widetilde{\Omega}_{\mathbb{P}^{1} \times X}^{\bullet}$ is twisted as $g \cdot \nu^{*}(\omega)=\nu^{*}\left(\overline{\nu^{*}(g)} \cdot \omega\right)$ for any function $g$ and any section $\omega$ of $\widetilde{\Omega}_{\mathbb{P}^{1} \times X}^{\bullet}$. Then we have the $C^{\infty}$-isomorphism $\Phi_{\nu}: \nu^{*} \widetilde{\Omega}_{\mathbb{P}^{1} \times X}^{\bullet} \simeq$ $\widetilde{\Omega}_{\mathbb{P}^{1} \times X}^{\bullet}$ given by the complex conjugate and the ordinary pull back

$$
\Phi_{\nu}\left(\nu^{*} \omega\right)=\overline{\nu^{*}(\omega)} .
$$

It is easy to check that $\Phi_{\nu} \circ \nu^{*}\left(\widetilde{\mathbb{D}}_{X}^{\Delta}\right)=\widetilde{\mathbb{D}}_{X}^{\Delta} \circ \Phi_{\nu}$. Similar relations hold for $\mathbb{D}_{X}^{\Delta}$ and $d_{\mathbb{P} 1}$. If we are given an additional bundle $\mathcal{F}$, the induced isomorphism $\mathcal{F} \otimes$ $\nu^{*}\left(\widetilde{\Omega}_{\mathbb{P}^{1} \times X}^{\bullet}\right) \simeq \mathcal{F} \otimes \widetilde{\Omega}_{\mathbb{P}^{1} \times X}^{\bullet}$ is also denoted by $\Phi_{\nu}$.

\subsubsection{Definitions and some remarks}

Variation of twistor structure. Let $V$ be a $C^{\infty}$-vector bundle on $\mathbb{P}^{1} \times X$. We use the same symbol to denote the associated sheaf of $C^{\infty}$-sections. A $\mathbb{P}^{1}$ holomorphic structure of $V$ is defined to be a differential operator

$$
d_{\mathbb{P}^{1}, V}^{\prime \prime}: V \rightarrow V \otimes \pi^{*} \Omega_{\mathbb{P}^{1}}^{0,1}
$$

satisfying (i) $d_{\mathbb{P}^{1}, V}^{\prime \prime}(f \cdot s)=f \cdot d_{\mathbb{P}^{1}, V}^{\prime \prime}(s)+\bar{\partial}_{\mathbb{P}^{1}}(f) \cdot s$ for any $C^{\infty}$-function $f$ and any section $s$ of $V$, (ii) $d_{\mathbb{P}^{1}, V}^{\prime \prime} \circ d_{\mathbb{P}^{1}, V}^{\prime \prime}=0$. The tuple $\left(V, d_{\mathbb{P}^{1}, V}^{\prime \prime}\right)$ is then called a $\mathbb{P}^{1}$ holomorphic vector bundle. A $T \widetilde{T}$-structure on $\left(V, d_{\mathbb{P}^{1}, V}^{\prime \prime}\right)$ is a differential operator

$$
\mathbb{D}_{V}^{\triangle}: V \rightarrow V \otimes \xi \Omega_{X}^{1}
$$

such that (i) $\mathbb{D}_{V}^{\Delta}(f \cdot s)=f \cdot \mathbb{D}_{V}^{\Delta}(s)+\mathbb{D}_{X}^{\Delta}(f) \cdot s$ for any $C^{\infty}$-function $f$ and any section $s$ of $V$, (ii) $\left(d_{\mathbb{P}^{1}, V}^{\prime \prime}+\mathbb{D}_{V}^{\Delta}\right)^{2}=0$. The tuple $\left(V, d_{\mathbb{P}^{1}, V}^{\prime \prime}, \mathbb{D}_{V}^{\Delta}\right)$ is then called a 
$T \widetilde{T}$-structure in [10, or a variation of $\mathbb{P}^{1}$-holomorphic vector bundle in 22 . In this section, we prefer to call it a variation of twistor structure. If $X$ is a point, it is just a holomorphic vector bundle on $\mathbb{P}^{1}$, and is called a twistor structure.

Remark 4. We will often omit to specify $d_{\mathbb{P}^{1}, V}^{\prime \prime}$ when we consider $\mathbb{P}^{1}$-holomorphic bundles or variations of $\mathbb{P}^{1}$-holomorphic bundle (variations of twistor structure).

Variation of integrable twistor structure. A $T \widetilde{T} E$-structure on $V$ is a differential operator

$$
\widetilde{\mathbb{D}}_{V}^{\triangle}: V \rightarrow V \otimes \widetilde{\Omega}_{\mathbb{P}^{1} \times X}^{1}
$$

satisfying (i) $\widetilde{\mathbb{D}}_{V}^{\triangle}(f \cdot s)=\widetilde{\mathbb{D}}_{X}^{\triangle}(f) \cdot s+f \cdot \widetilde{\mathbb{D}}_{V}^{\triangle}(s)$ for a $C^{\infty}$-function $f$ and a section $s$ of $V$, (ii) $\widetilde{\mathbb{D}}_{V}^{\triangle} \circ \widetilde{\mathbb{D}}_{V}^{\triangle}=0$. The tuple $\left(V, \widetilde{\mathbb{D}}_{V}^{\triangle}\right)$ is then called a variation of integrable twistor structure. Its restriction to $\left(\mathbb{P}^{1} \backslash\{0, \infty\}\right) \times X$ gives a flat bundle. If $X$ is a point, it is equivalent to a holomorphic vector bundle $V$ on $\mathbb{P}^{1}$ with a meromorphic connection $\nabla$ which admits a pole at $\{0, \infty\}$ with order at most 2 , i.e.,

$$
\nabla(V) \subset V \otimes \Omega^{1}(2 \cdot\{0, \infty\}) .
$$

In this case, it is simply called an integrable twistor structure.

Morphisms. A morphism of variations of (integrable) twistor structure is defined to be a morphism of the associated sheaves of $C^{\infty}$-sections, compatible with the associated differential operators. If $X$ is a point, a morphism of twistor structures is an $\mathcal{O}_{\mathbb{P}^{1}}$-morphism, and a morphism of integrable twistor structures is an $\mathcal{O}_{\mathbb{P}^{1}}$ morphism compatible with the meromorphic connections.

Some functoriality. Let $\left(V, \widetilde{\mathbb{D}}_{V}^{\triangle}\right)$ be a variation of integrable twistor structure. Let $f: Y \rightarrow X$ be a holomorphic map of complex manifolds. Then we have the naturally induced variation of integrable twistor structure $f^{*}\left(V, \widetilde{\mathbb{D}}_{V}^{\triangle}\right)$ as in the case of ordinary connections.

Let $\nu: \mathbb{P}^{1} \rightarrow \mathbb{P}^{1}$ be a diffeomorphism satisfying one of (A1) or (A2) above. Then $\nu^{*} V$ is naturally equipped with a $T \widetilde{T} E$-structure $\widetilde{\mathbb{D}}_{\nu^{*} V}^{\triangle}$ given as follows:

$$
\widetilde{\mathbb{D}}_{\nu^{*} V}^{\triangle}\left(\Phi_{\nu}\left(\nu^{*} s\right)\right)=\Phi_{\nu}\left(\nu^{*}\left(\widetilde{\mathbb{D}}_{V}^{\triangle}(s)\right)\right) .
$$

Here, $s$ denotes a section of $V \otimes \widetilde{\Omega}_{X}^{\bullet}$, and $\Phi_{\nu}: \nu^{*} V \otimes \nu^{*} \widetilde{\Omega}^{\bullet} \simeq \nu^{*} V \otimes \widetilde{\Omega}^{\bullet}$ is as in Subsection 2.1.1.

Similarly, we also have the pull back of a variation of twistor structure via $f$ and $\nu$ as above.

Pure and mixed. Let $\left(V, d_{\mathbb{P}^{1}, V}^{\prime \prime}\right)$ be a $\mathbb{P}^{1}$-holomorphic vector bundle on $\mathbb{P}^{1} \times X$. It is called pure of weight $w$ if the restrictions $V_{P}:=\left(V, d_{\mathbb{P}^{1}, V}^{\prime \prime}\right)_{\mid \mathbb{P}^{1} \times\{P\}}$ are pure twistor structures of weight $w$ for any $P \in X$, i.e., $V_{P}$ are isomorphic to direct sums 
of $\mathcal{O}_{\mathbb{P}^{1}}(w)$. A variation of (integrable) twistor structure is called pure of weight $w$ if the underlying $\mathbb{P}^{1}$-holomorphic vector bundle is pure of weight $w$.

Let $W$ be an increasing filtration of $V$ by vector subbundles indexed by integers. We say that $W$ is $\mathbb{P}^{1}$-holomorphic if each $W_{n}$ is preserved by $d_{\mathbb{P}^{1}, V}^{\prime \prime}$. We have the induced $\mathbb{P}^{1}$-holomorphic vector bundles $\operatorname{Gr}_{n}^{W}\left(V, d_{\mathbb{P}^{1}, V}^{\prime \prime}\right)$. Then $\left(V, d_{\mathbb{P}^{1}}^{\prime \prime}, W\right)$ is called mixed if each $\operatorname{Gr}_{n}^{W}\left(V, d_{\mathbb{P}^{1}, V}^{\prime \prime}\right)$ is pure of weight $n$. When $\left(V, d_{\mathbb{P}^{1}}^{\prime \prime}\right)$ is equipped with $T \widetilde{T}$-structure $\mathbb{D}_{V}^{\triangle}$ (resp. $T \widetilde{T} E$-structure $\widetilde{\mathbb{D}}_{V}^{\triangle}$ ), we say that $W$ is $\mathbb{D}_{V}^{\triangle}$-flat (resp. $\widetilde{\mathbb{D}}_{V}^{\triangle}$-flat), or more simply flat, if each $W_{n}$ is preserved by the operator. In that case, $\left(V, d_{\mathbb{P}^{1}, V}^{\prime \prime}, \mathbb{D}_{V}^{\triangle}, W\right)$ (resp. $\left.\left(V, \widetilde{\mathbb{D}}_{V}^{\triangle}, W\right)\right)$ is called mixed if $\left(V, d_{\mathbb{P}^{1}}^{\prime \prime}, W\right)$ is mixed.

New supersymmetric index. Let $(V, \nabla)$ be a pure integrable twistor structure of weight 0 . We have a global trivialization $V \simeq \mathcal{O}_{\mathbb{P}^{1}}^{\oplus r}$, which is uniquely determined up to obvious ambiguity. Let $d$ denote the natural connection of $\mathcal{O}_{\mathbb{P 1}}^{\oplus r}$. Then we have the decomposition

$$
\nabla=d+\left(\lambda^{-1} \mathcal{U}_{1}-\mathcal{Q}-\lambda \mathcal{U}_{2}\right) \frac{d \lambda}{\lambda}
$$

where $\mathcal{U}_{1}, \mathcal{U}_{2}, \mathcal{Q} \in H^{0}\left(\mathbb{P}^{1}, \operatorname{End}(V)\right)$. The operator $\mathcal{Q}$ is called the new supersymmetric index. If $(V, \nabla)$ is equipped with a polarization (Subsection 2.1.4), then $\mathcal{U}_{2}$ and $\mathcal{U}_{1}$ are adjoint with respect to the induced hermitian metric, as observed by Hertling and Sabbah.

If we are given a variation of polarized pure integrable twistor structure, we obtain the family of such operators.

2.1.3. Simple examples. We recall some simplest examples of integrable pure twistor structures.

Example (Tate object). Let $\mathbb{T}(w)$ be a Tate object in the theory of twistor structures. (See [32] and Subsection 3.3.1 of [22].) It is isomorphic to $\mathcal{O}_{\mathbb{P}^{1}}(-2 w)$, and it is equipped with the distinguished frames

$$
\mathbb{T}(w)_{\mid \mathbb{C}_{\lambda}}=\mathcal{O}_{\mathbb{C}_{\lambda}} \cdot t_{0}^{(w)}, \quad \mathbb{T}(w)_{\mid \mathbb{C}_{\mu}}=\mathcal{O}_{\mathbb{C}_{\mu}} \cdot t_{\infty}^{(w)}, \quad \mathbb{T}(w)_{\mid \mathbb{C}_{\lambda}^{*}}=\mathcal{O}_{\mathbb{C}_{\lambda}^{*}} \cdot t_{1}^{(w)}
$$

The transformation is given by

$$
t_{0}^{(w)}=(\sqrt{-1} \lambda)^{w} \cdot t_{1}^{(w)}, \quad t_{\infty}^{(w)}=(-\sqrt{-1} \mu)^{w} \cdot t_{1}^{(w)} .
$$

In particular, $(\sqrt{-1} \lambda)^{-2 w} t_{0}^{(w)}=t_{\infty}^{(w)}$. We have the meromorphic connection $\nabla_{\mathbb{T}(w)}$ on $\mathbb{T}(w)$ determined by $\nabla_{\mathbb{T}(w)} t_{1}^{(w)}=0$, which implies

$$
\nabla_{\mathbb{T}(w)} t_{0}^{(w)}=t_{0}^{(w)} \cdot\left(w \frac{d \lambda}{\lambda}\right), \quad \nabla_{\mathbb{T}(w)} t_{\infty}^{(w)}=t_{\infty}^{(w)} \cdot\left(w \frac{d \mu}{\mu}\right)
$$


In the following, the connection on $\mathbb{T}(w)$ is always given as above, and hence we often omit to specify it explicitly.

We may identify $\mathbb{T}(w)$ with $\mathcal{O}_{\mathbb{P}^{1}}(-w \cdot 0-w \cdot \infty)$ by the correspondence $t_{1}^{(w)} \leftrightarrow 1$, up to constant multiplication. In particular, we implicitly use the identification of $\mathbb{T}(0)$ with $\mathcal{O}_{\mathbb{P}^{1}}$ by $t_{1}^{(0)} \leftrightarrow 1$. We will also implicitly use the identification $\mathbb{T}(m) \otimes$ $\mathbb{T}(n) \simeq \mathbb{T}(m+n)$ given by $t_{a}^{(m)} \otimes t_{a}^{(n)} \leftrightarrow t_{a}^{(m+n)}$.

Example. In Subsection 3.3.2 of [22], we considered a line bundle $\mathcal{O}(p, q)$ on $\mathbb{P}^{1}$, which is isomorphic to $\mathcal{O}_{\mathbb{P}^{1}}(p+q)$ and equipped with the distinguished frames

$$
\mathcal{O}(p, q)_{\mid \mathbb{C}_{\lambda}}=\mathcal{O}_{\mathbb{C}_{\lambda}} \cdot f_{0}^{(p, q)}, \quad \mathcal{O}(p, q)_{\mid \mathbb{C}_{\mu}}=\mathcal{O}_{\mathbb{C}_{\mu}} \cdot f_{\infty}^{(p, q)}, \quad \mathcal{O}(p, q)_{\mid \mathbb{C}_{\lambda}^{*}}=\mathcal{O}_{\mathbb{C}_{\lambda}^{*}} \cdot f_{1}^{(p, q)}
$$

The transformation is given by

$$
f_{0}^{(p, q)}=(\sqrt{-1} \lambda)^{-p} \cdot f_{1}^{(p, q)}, \quad f_{\infty}^{(p, q)}=(-\sqrt{-1} \mu)^{-q} \cdot f_{1}^{(p, q)} .
$$

In particular, $(\sqrt{-1} \lambda)^{p+q} f_{0}^{(p, q)}=f_{\infty}^{(p, q)}$. We have the meromorphic connection $\nabla_{\mathcal{O}(p, q)}$ on $\mathcal{O}(p, q)$ determined by $\nabla_{\mathcal{O}(p, q)} f_{1}^{(p, q)}=0$, which implies

$$
\nabla_{\mathcal{O}(p, q)} f_{0}^{(p, q)}=f_{0}^{(p, q)} \cdot\left(-p \frac{d \lambda}{\lambda}\right), \quad \nabla_{\mathcal{O}(p, q)} f_{\infty}^{(p, q)}=f_{\infty}^{(p, q)} \cdot\left(-q \frac{d \mu}{\mu}\right) .
$$

In the following, the connection on $\mathcal{O}(p, q)$ is always given as above, and hence we will often omit to specify it explicitly.

We may naturally identify $\mathcal{O}(p, q)$ with $\mathcal{O}_{\mathbb{P}^{1}}(p \cdot 0+q \cdot \infty)$ by the correspondence $f_{1}^{(p, q)} \leftrightarrow 1$, up to constant multiplication. We will implicitly use the identification $\mathcal{O}(p, q) \otimes \mathcal{O}\left(p^{\prime}, q^{\prime}\right) \simeq \mathcal{O}\left(p+p^{\prime}, q+q^{\prime}\right)$ given by $f_{a}^{(p, q)} \otimes f_{a}^{\left(p^{\prime}, q^{\prime}\right)} \leftrightarrow f_{a}^{\left(p+p^{\prime}, q+q^{\prime}\right)}$. We will also implicitly identify $\mathbb{T}(w)$ with $\mathcal{O}(-w,-w)$ by $t_{a}^{(w)}=f_{a}^{(-w,-w)}$ for $a=0,1, \infty$.

Let $X$ be a complex manifold. We have the pull backs of $\mathbb{T}(w)$ and $\mathcal{O}(p, q)$ via the map from $X$ to a point. They are denoted by $\mathbb{T}(w)_{X}$ and $\mathcal{O}(p, q)_{X}$, respectively. We will often omit to denote $X$, if there is no risk of confusion.

2.1.4. Polarization. Recall that we have the isomorphism ([22])

$$
\iota_{\mathbb{T}(w)}: \sigma^{*} \mathbb{T}(w) \simeq \mathbb{T}(w),
$$

given by the natural identification $\sigma^{*} \mathcal{O}(-w \cdot 0-w \cdot \infty) \simeq \mathcal{O}(-w \cdot 0-w \cdot \infty)$ via $\sigma^{*}(1) \leftrightarrow 1$, or equivalently,

$$
\sigma^{*} t_{1}^{(w)} \leftrightarrow t_{1}^{(w)}, \quad \sigma^{*} t_{\infty}^{(w)} \leftrightarrow(-1)^{w} \cdot t_{0}^{(w)}, \quad \sigma^{*} t_{0}^{(w)} \leftrightarrow(-1)^{w} \cdot t_{\infty}^{(w)} .
$$

It preserves the flat connections, i.e., $\iota_{\mathbb{T}(w)}: \sigma^{*}\left(\mathbb{T}(w), \nabla_{\mathbb{T}(w)}\right) \simeq\left(\mathbb{T}(w), \nabla_{\mathbb{T}(w)}\right)$.

For a variation of integrable twistor structure $\left(V, \widetilde{\mathbb{D}}_{V}^{\triangle}\right)$ on $\mathbb{P}^{1} \times X$, a morphism

$$
\mathcal{S}:\left(V, \widetilde{\mathbb{D}}_{V}^{\triangle}\right) \otimes \sigma^{*}\left(V, \widetilde{\mathbb{D}}_{V}^{\triangle}\right) \rightarrow \mathbb{T}(-w)_{X}
$$


is called a pairing of weight $w$ if it is $(-1)^{w}$-symmetric in the following sense:

$$
\iota_{\mathbb{T}(-w)} \circ \sigma^{*} \mathcal{S}=(-1)^{w} \mathcal{S} \circ \text { exchange }: \sigma^{*} V \otimes V \rightarrow \mathbb{T}(-w)_{X} .
$$

Here, exchange denotes the natural morphism $\sigma^{*} V \otimes V \rightarrow V \otimes \sigma^{*} V$ induced by the exchange of components. Similarly, we have the notion of pairing of weight $w$ for variations of twistor structure.

Definition 2.1. Let $\left(V, \widetilde{\mathbb{D}}_{V}^{\triangle}\right)$ be a variation of integrable pure twistor structure of weight $w$ on $\mathbb{P}^{1} \times X$. Let $\mathcal{S}:\left(V, \widetilde{\mathbb{D}}_{V}^{\triangle}\right) \otimes \sigma^{*}\left(V, \widetilde{\mathbb{D}}_{V}^{\triangle}\right) \rightarrow \mathbb{T}(-w)_{X}$ be a pairing of weight $w$. We say that $\mathcal{S}$ is a polarization of $\left(V, \widetilde{\mathbb{D}}_{V}^{\triangle}\right)$ if $\mathcal{S}_{P}:=\mathcal{S}_{\mid \mathbb{P}^{1} \times\{P\}}$ is a polarization of $V_{P}:=\left(V, d_{\mathbb{P}^{1}}^{\prime \prime}\right)_{\mid \mathbb{P}^{1} \times\{P\}}$ for each $P \in X$, that is, the following holds:

- If $w=0$, the induced Hermitian pairing $H^{0}\left(\mathcal{S}_{P}\right)$ on $H^{0}\left(\mathbb{P}^{1}, V_{P}\right)$ is positive definite.

- In the general case, the induced pairing $\mathcal{S}_{P} \otimes \mathcal{S}_{0,-w}$ on $V_{P} \otimes \mathcal{O}(0,-w)$ is a polarization of the pure twistor structure. (See Example 2 below for $\mathcal{S}_{0,-w}$.)

The notion of polarization for variations of pure twistor structure is defined in a similar way.

Example 1. The identification $\iota_{\mathbb{T}(w)}$ induces a flat morphism $\mathcal{S}_{\mathbb{T}(w)}: \mathbb{T}(w) \otimes$ $\sigma^{*} \mathbb{T}(w) \rightarrow \mathbb{T}(2 w)$, which is a polarization of $\mathbb{T}(w)$ of weight $-2 w$. (See [22.)

Example 2. More generally, a flat isomorphism $\iota_{(p, q)}: \sigma^{*} \mathcal{O}(p, q) \simeq \mathcal{O}(q, p)$ in [22] is given by the correspondence $\sigma^{*} f_{1}^{(p, q)} \mapsto(\sqrt{-1})^{q-p} f_{1}^{(q, p)}$, which implies

$$
\sigma^{*} f_{0}^{(p, q)} \mapsto(\sqrt{-1})^{p+q} f_{\infty}^{(q, p)}, \quad \sigma^{*} f_{\infty}^{(p, q)} \mapsto(-\sqrt{-1})^{p+q} f_{0}^{(q, p)} .
$$

Hence, we obtain the morphism $\mathcal{S}_{p, q}: \mathcal{O}(p, q) \otimes \sigma^{*} \mathcal{O}(p, q) \rightarrow \mathbb{T}(-p-q)$, which is a polarization of weight $p+q$. (See [22].)

\subsubsection{Real structure and twistor-TERP structure}

Definition 2.2. A real structure on a variation of integrable twistor structure $\left(V, \widetilde{\mathbb{D}}_{V}^{\triangle}\right)$ is defined to be an isomorphism

$$
\kappa: \gamma^{*}\left(V, \widetilde{\mathbb{D}}_{V}^{\triangle}\right) \simeq\left(V, \widetilde{\mathbb{D}}_{V}^{\triangle}\right)
$$

such that $\gamma^{*}(\kappa) \circ \kappa=$ id.

We fix the real structure $\kappa_{\mathbb{T}(w)}$ on $\mathbb{T}(w)$ given by the correspondence

$$
\gamma^{*} t_{1}^{(w)} \leftrightarrow t_{1}^{(w)}, \quad \gamma^{*} t_{0}^{(w)} \leftrightarrow t_{\infty}^{(w)}, \quad \gamma^{*} t_{\infty}^{(w)} \leftrightarrow t_{0}^{(w)}
$$


Definition 2.3. Let $\left(V, \widetilde{\mathbb{D}}_{V}^{\triangle}\right)$ be a variation of integrable twistor structure with a pairing $\mathcal{S}$ of weight $w$ and a real structure $\kappa$. We say that $\kappa$ and $\mathcal{S}$ are compatible if the following diagram is commutative:

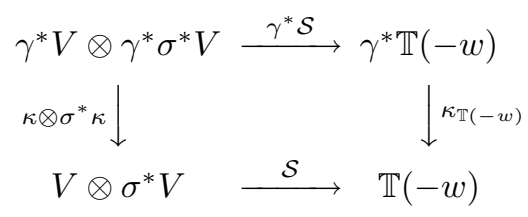

That is, $\kappa_{\mathbb{T}(-w)} \circ \gamma^{*} \mathcal{S}=\mathcal{S} \circ\left(\kappa \otimes \sigma^{*} \kappa\right)$. In that case, we also say that $\kappa$ is a real structure on $\left(V, \widetilde{\mathbb{D}}_{V}^{\Delta}, \mathcal{S}\right)$, or that $\mathcal{S}$ is a pairing on $\left(V, \widetilde{\mathbb{D}}_{V}^{\Delta}, \kappa\right)$ with weight $w$.

Definition 2.4. Let $\left(V, \widetilde{\mathbb{D}}_{V}^{\triangle}\right)$ be a variation of integrable twistor structure with a pairing $\mathcal{S}$ of weight $w$ and a real structure $\kappa$. The tuple $\left(V, \widetilde{\mathbb{D}}_{V}^{\Delta}, \mathcal{S}, \kappa,-w\right)$ is called a variation of twistor-TERP structure if (i) $\mathcal{S}$ is perfect, (ii) $\mathcal{S}$ and $\kappa$ are compatible.

If $X$ is a point, it is called a twistor-TERP structure.

It is easy to observe that twistor-TERP structure is just an expression of TERP structure [10 in terms of twistor structures, which we will explain in Subsection 2.1.8.

Definition 2.5. A variation of twistor-TERP structure $\left(V, \widetilde{\mathbb{D}}_{V}^{\triangle}, \mathcal{S}, \kappa, w\right)$ is called pure if $\left(V, \widetilde{\mathbb{D}}_{V}^{\Delta}\right)$ is pure of weight $-w$. It is called polarized if $\left(V, \widetilde{\mathbb{D}}_{V}^{\Delta}, \mathcal{S}\right)$ is polarized.

Remark 5. If a variation of twistor-TERP structure $\left(V, \widetilde{\mathbb{D}}_{V}^{\Delta}, \mathcal{S}, \kappa,-w\right)$ is pure, we also say that $\left(V, \widetilde{\mathbb{D}}_{V}^{\Delta}, \mathcal{S}, \kappa\right)$ is a variation of pure twistor-TERP structure of weight $w$.

Example. A Tate object $\left(\mathbb{T}(w), \nabla_{\mathbb{T}(w)}, \mathcal{S}_{\mathbb{T}(w)}, \kappa_{\mathbb{T}(w)}, 2 w\right)$ is a pure polarized twistor-TERP structure.

\subsubsection{Gluing construction}

Variation of integrable twistor structure. We can describe a variation of integrable twistor structure as gluing. We set $\mathcal{X}:=\mathbb{C}_{\lambda} \times X, \mathcal{X}^{0}:=\{0\} \times X$, $\mathcal{X}^{\dagger}:=\mathbb{C}_{\mu} \times X^{\dagger}$ and $\mathcal{X}^{\dagger 0}:=\{0\} \times X^{\dagger}$.

Let $V_{0}$ be a holomorphic vector bundle on $\mathcal{X}$ with a meromorphic flat connection (TE-structure [10])

$$
\nabla_{V_{0}}: V_{0} \rightarrow V_{0} \otimes \Omega_{\mathcal{X}}^{1,0}\left(\log \mathcal{X}^{0}\right) \otimes \mathcal{O}_{\mathcal{X}}\left(\mathcal{X}^{0}\right) .
$$

We use the same symbol to denote the associated differential operator $V_{0} \rightarrow$ $V_{0} \otimes \widetilde{\Omega}_{\mathcal{X}}^{1}$ in the $C^{\infty}$-category. (The holomorphic structure $d_{V_{0}}^{\prime \prime}$ is also included.) 
Let $V_{\infty}$ be a holomorphic vector bundle on $\mathcal{X}^{\dagger}$ with a meromorphic flat connection $(\widetilde{T} E$-structure [10])

$$
\nabla_{V_{\infty}}: V_{\infty} \rightarrow V_{\infty} \otimes \Omega_{\mathcal{X}^{\dagger}}^{1,0}\left(\log \mathcal{X}^{\dagger 0}\right) \otimes \mathcal{O}_{\mathcal{X}^{\dagger}}\left(\mathcal{X}^{\dagger 0}\right) .
$$

We use the same symbol to denote the associated differential operator $V_{\infty} \rightarrow$ $V_{\infty} \otimes \widetilde{\Omega}_{\mathcal{X}^{\dagger}}^{1}$ in the $C^{\infty}$-category. Assume that we are given an isomorphism $\Phi$ of $C^{\infty}$-flat bundles:

$$
\Phi:\left(V_{0}, \nabla_{V_{0}}\right)_{\mid \mathbb{C}_{\lambda}^{*} \times X} \simeq\left(V_{\infty}, \nabla_{V_{\infty}}\right)_{\mathbb{C}_{\mu}^{*} \times X^{\dagger}}
$$

We obtain the $C^{\infty}$-vector bundle $V$ on $\mathbb{P}^{1} \times X$ by gluing $V_{0}$ and $V_{\infty}$ via $\Phi$. Since $\Phi$ is flat, $\nabla_{V_{0}}$ and $\nabla_{V_{\infty}}$ induce the $T \widetilde{T} E$-structure $\widetilde{\mathbb{D}}_{V}^{\Delta}: V \rightarrow V \otimes \widetilde{\Omega}_{\mathbb{P}^{1} \times X}^{1}$. Thus, we obtain a variation of integrable twistor structure $\left(V, \widetilde{\mathbb{D}}_{V}^{\Delta}\right)$.

Conversely, we naturally obtain a tuple of $\left(V_{0}, \nabla_{V_{0}}\right),\left(V_{\infty}, \nabla_{V_{\infty}}\right)$ and $\Phi$ as above from a variation of integrable twistor structure $\left(V, \widetilde{\mathbb{D}}_{V}^{\Delta}\right)$ as the restriction to $\mathcal{X}$ and $\mathcal{X}^{\dagger}$, respectively. In this situation, we set

$$
\text { Glue }\left(\left(V_{0}, \nabla_{V_{0}}\right),\left(V_{\infty}, \nabla_{V_{\infty}}\right), \Phi\right):=\left(V, \widetilde{\mathbb{D}}_{V}^{\Delta}\right) .
$$

Pairing and real structure. Note that we have the natural isomorphisms $\nu^{*} \widetilde{\Omega}_{\mathcal{X}^{\dagger}}^{1}$ $\simeq \widetilde{\Omega}_{\mathcal{X}}^{1}$ and $\nu^{*} \widetilde{\Omega}_{\mathcal{X}}^{1} \simeq \widetilde{\Omega}_{\mathcal{X}^{\dagger}}^{1}$ for an anti-holomorphic diffeomorphism $\nu: \mathbb{C}_{\lambda} \rightarrow \mathbb{C}_{\mu}$ or $\mathbb{C}_{\mu} \rightarrow \mathbb{C}_{\lambda}$, as in the case of $\widetilde{\Omega}_{\mathbb{P}^{1} \times X}^{1}$. Let $V_{0}$ be a holomorphic vector bundle on $\mathcal{X}$ with a $T E$-structure $\nabla_{V_{0}}$. By the above isomorphisms, $\gamma^{*} V_{0}$ and $\sigma^{*} V_{0}$ are naturally equipped with $\widetilde{T} E$-structures $\nabla_{\gamma^{*} V_{0}}$ and $\nabla_{\sigma^{*} V_{0}}$. Similarly, if we are given a holomorphic vector bundle $V_{\infty}$ on $\mathcal{X}^{\dagger}$ with $\widetilde{T} E$-structure, $\sigma^{*} V_{\infty}$ and $\gamma^{*} V_{\infty}$ are naturally equipped with $T E$-structures. We remark that there exist natural isomorphisms

$$
\begin{aligned}
& \operatorname{Glue}\left(\gamma^{*}\left(V_{\infty}, \nabla_{V_{\infty}}\right), \gamma^{*}\left(V_{0}, \nabla_{V_{0}}\right), \gamma^{*} \Phi^{-1}\right) \simeq \gamma^{*} \operatorname{Glue}\left(\left(V_{0}, \nabla_{V_{0}}\right),\left(V_{\infty}, \nabla_{V_{\infty}}\right), \Phi\right), \\
& \operatorname{Glue}\left(\sigma^{*}\left(V_{\infty}, \nabla_{V_{\infty}}\right), \sigma^{*}\left(V_{0}, \nabla_{V_{0}}\right), \sigma^{*} \Phi^{-1}\right) \simeq \sigma^{*} \operatorname{Glue}\left(\left(V_{0}, \nabla_{V_{0}}\right),\left(V_{\infty}, \nabla_{V_{\infty}}\right), \Phi\right) .
\end{aligned}
$$

A real structure on a variation of integrable twistor structure corresponds to a pair of isomorphisms

$$
\kappa_{0}: \gamma^{*}\left(V_{\infty}, \nabla_{V_{\infty}}\right) \simeq\left(V_{0}, \nabla_{V_{0}}\right), \quad \kappa_{\infty}: \gamma^{*}\left(V_{0}, \nabla_{V_{0}}\right) \simeq\left(V_{\infty}, \nabla_{V_{\infty}}\right)
$$

such that (i) $\gamma^{*} \kappa_{0}=\kappa_{\infty}^{-1}$, (ii) the following commutativity holds on $\mathbb{C}_{\lambda}^{*} \times X$ :

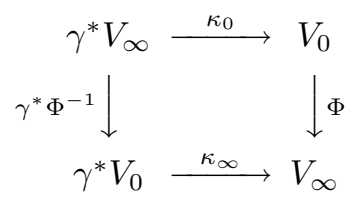


A pairing of weight $w$ corresponds to morphisms

$$
\begin{array}{r}
\mathcal{S}_{0}:\left(V_{0}, \nabla_{V_{0}}\right) \otimes \sigma^{*}\left(V_{\infty}, \nabla_{V_{\infty}}\right) \rightarrow \mathbb{T}(-w)_{\mid \mathcal{X}}, \\
\mathcal{S}_{\infty}:\left(V_{\infty}, \nabla_{V_{\infty}}\right) \otimes \sigma^{*}\left(V_{0}, \nabla_{V_{0}}\right) \rightarrow \mathbb{T}(-w)_{\mid \mathcal{X}^{\dagger}}
\end{array}
$$

such that (i) $\iota_{\mathbb{T}(-w)} \circ \sigma^{*} \mathcal{S}_{\infty}=(-1)^{w} \mathcal{S}_{0} \circ$ exchange, (ii) it is compatible with the gluing. Compatibility of $\mathcal{S}$ and $\kappa$ means $\kappa_{\mathbb{T}(-w)} \circ \gamma^{*} \mathcal{S}_{\infty}=\mathcal{S}_{0} \circ\left(\kappa_{0} \otimes \sigma^{*} \kappa_{\infty}\right)$.

Variation of twistor structure. The above gluing description is essentially the same as that for a variation of twistor structure in 32 , which we recall in the following. See also $\left[22\right.$. We have the decomposition $\widetilde{\Omega}_{\mathcal{X}}^{1}=\xi \widetilde{\Omega}_{X \mid \mathcal{X}}^{1} \oplus \widetilde{\Omega}_{\mathbb{C}_{\lambda}}^{1}$ into the $X$ direction and the $\mathbb{C}_{\lambda}$-direction. Let $d_{X}$ denote the restriction of the exterior derivative to the $X$-direction. Similarly, we have the decomposition $\widetilde{\Omega}_{\mathcal{X}^{\dagger}}^{1}=\xi \widetilde{\Omega}_{X \mid \mathcal{X}^{\dagger}}^{1} \oplus \widetilde{\Omega}_{\mathbb{C}_{\mu}}^{1}$, and the restriction of $\widetilde{\mathbb{D}}_{X}^{\dagger f}$ to the $X$-direction is denoted by $d_{X^{\dagger}}$. The notions of $\mathbb{C}_{\lambda}$-holomorphic bundles or $\mathbb{C}_{\mu}$-holomorphic bundles are defined as in the case of $\mathbb{P}^{1}$-holomorphic bundles.

Let $\left(V_{0}, d_{\mathbb{C}_{\lambda}, V_{0}}^{\prime \prime}\right)$ be a $\mathbb{C}_{\lambda}$-holomorphic bundle on $\mathcal{X}$. A $T$-structure [10] on $V_{0}$ is a differential operator

$$
\mathbb{D}_{V_{0}}^{f}: V_{0} \rightarrow V_{0} \otimes \xi \Omega_{X \mid \mathcal{X}}^{1}
$$

satisfying (i) $\mathbb{D}_{V_{0}}^{f}(f \cdot s)=d_{X} f \cdot s+f \cdot \mathbb{D}_{V_{0}}^{f}(s)$ for any function $f$ and any section $s$ of $V$, (ii) $\left(d_{\mathbb{C}_{\lambda}, V_{0}}^{\prime \prime}+\mathbb{D}_{V_{0}}^{f}\right)^{2}=0$. Let $\left(V_{\infty}, d_{\mathbb{C}_{\mu}, V_{\infty}}^{\prime \prime}\right)$ be a $\mathbb{C}_{\mu}$-holomorphic vector bundle on $\mathcal{X}^{\dagger}$. A $\widetilde{T}$-structure [10] is defined to be a differential operator

$$
\mathbb{D}_{V_{\infty}}^{\dagger f}: V_{\infty} \rightarrow V_{\infty} \otimes \xi \Omega_{X \mid \mathcal{X}^{\dagger}}^{1}
$$

satisfying conditions similar to (i) and (ii) above. Assume that we are given an isomorphism

$$
\Phi:\left(V_{0}, d_{\mathbb{C}_{\lambda}, V_{0}}^{\prime \prime}, \mathbb{D}_{V_{0}}^{f}\right)_{\mid \mathbb{C}_{\lambda}^{*} \times X} \simeq\left(V_{\infty}, d_{\mathbb{C}_{\mu}, V_{\infty}}^{\prime \prime}, \mathbb{D}_{V_{\infty}}^{\dagger f}\right)_{\mid \mathbb{C}_{\mu}^{*} \times X^{\dagger}}
$$

We obtain the $C^{\infty}$-vector bundle $V$ on $\mathbb{P}^{1} \times X$ by gluing $V_{0}$ and $V_{\infty}$ via $\Phi$. By the condition 2.2$), d_{\mathbb{C}_{\lambda}, V_{0}}^{\prime \prime}$ and $d_{\mathbb{C}_{\mu}, V_{\infty}}^{\prime \prime}$ give a $\mathbb{P}^{1}$-holomorphic structure $d_{\mathbb{P}^{1}, V}^{\prime \prime}$, and $\mathbb{D}_{V_{0}}^{f}$ and $\mathbb{D}_{V_{\infty}}^{\dagger f}$ induce the $T \widetilde{T}$-structure $\mathbb{D}_{V}^{\triangle}$. Thus, we obtain a variation of twistor structure $\left(V, d_{\mathbb{P}^{1}, V}^{\prime \prime}, \mathbb{D}_{V}^{\triangle}\right)$.

Conversely, we obtain such a tuple of $\left(V_{0}, d_{\mathbb{C}_{\lambda}, V_{0}}^{\prime \prime}, \mathbb{D}_{V_{0}}^{f}\right),\left(V_{\infty}, d_{\mathbb{C}_{\mu}, V_{\infty}}^{\prime \prime}, \mathbb{D}_{V_{\infty}}^{\dagger}\right)$ and $\Phi$ from a variation of twistor structure $\left(V, d_{\mathbb{P}^{1}, V}^{\prime \prime}, \mathbb{D}_{V}^{\triangle}\right)$ as the restriction to $\mathcal{X}$ and $\mathcal{X}^{\dagger}$, respectively. In this situation, we set

$$
\text { Glue }\left(\left(V_{0}, d_{\mathbb{C}_{\lambda, V_{0}}}^{\prime \prime} \mathbb{D}_{V_{0}}^{f}\right),\left(V_{\infty}, d_{\mathbb{C}_{\mu}, V_{\infty}}^{\prime \prime}, \mathbb{D}_{V_{\infty}}^{\dagger f}\right), \Phi\right):=\left(V, \widetilde{\mathbb{D}}_{V}^{\triangle}\right)
$$


Remark 6 . Let $p_{\lambda}$ be the projection $\mathcal{X} \rightarrow X$. Under the natural isomorphism

$$
\xi \Omega_{X \mid \mathcal{X}}^{1}=\lambda^{-1} \cdot p_{\lambda}^{-1} \Omega_{X}^{1,0} \oplus p_{\lambda}^{-1} \Omega_{X}^{0,1} \simeq p_{\lambda}^{-1} \Omega_{X}^{1,0} \oplus p_{\lambda}^{-1} \Omega_{X}^{0,1}=p_{\lambda}^{-1} \Omega_{X}^{1},
$$

a $T$-structure $\mathbb{D}_{V_{0}}^{f}$ induces a holomorphic family of flat $\lambda$-connections $\mathbb{D}_{V_{0}}$. Similarly, a $\widetilde{T}$-structure on $\mathbb{D}_{V_{\infty}}^{\dagger f}$ naturally induces a holomorphic family of flat $\mu$ connections $\mathbb{D}_{V_{\infty}}^{\dagger}$. Hence, a variation of twistor structure is regarded as the gluing of families of $\lambda$-flat bundles and $\mu$-flat bundles.

2.1.7. Relation to harmonic bundles. We recall a fundamental equivalence due to Hertling and Sabbah. Let $X$ be a complex manifold. Let $\left(\mathcal{E}^{\triangle}, \widetilde{\mathbb{D}}^{\triangle}, \mathcal{S}\right)$ be a variation of pure polarized integrable twistor structure of weight 0 on $\mathbb{P}^{1} \times X$. By the equivalence between harmonic bundles and variations of pure polarized twistor structure due to Simpson [32, we have the underlying harmonic bundle $\left(E, \bar{\partial}_{E}, \theta, h\right)$ on $X$. Note that $\operatorname{End}\left(E_{\mid Q}\right)(Q \in X)$ is naturally identified with $H^{0}\left(\mathbb{P}^{1}, \operatorname{End}\left(\mathcal{E}_{\mid \mathbb{P}^{1} \times Q}^{\triangle}\right)\right)$, and hence it is equipped with the endomorphisms $\mathcal{U}_{Q}:=$ $\mathcal{U}_{1 Q}$ and $\mathcal{Q}_{Q}$ obtained as in 2.1. They give $C^{\infty}$-sections $\mathcal{U}$ and $\mathcal{Q}$ of $\operatorname{End}(E)$ satisfying the following equations:

$$
\begin{gathered}
\bar{\partial}_{E} \mathcal{U}=0, \quad[\mathcal{U}, \theta]=0, \quad \mathcal{Q}=\mathcal{Q}^{\dagger}, \\
\partial_{E} \mathcal{U}-[\theta, \mathcal{Q}]+\theta=0, \quad \partial_{E} \mathcal{Q}+\left[\theta, \mathcal{U}^{\dagger}\right]=0 .
\end{gathered}
$$

Here, $\mathcal{U}^{\dagger}$ and $\mathcal{Q}^{\dagger}$ denote the adjoint of $\mathcal{U}$ and $\mathcal{Q}$ with respect to $h$, respectively. Conversely, we obtain a variation of polarized pure integrable twistor structure $\left(\mathcal{E}^{\triangle}, \widetilde{\mathbb{D}}^{\triangle}, \mathcal{S}\right)$ from a harmonic bundle $\left(E, \bar{\partial}_{E}, \theta, h\right)$ with $\mathcal{U}$ and $\mathcal{Q}$ satisfying 2.3 and 2.4. Let $p: \mathbb{P}^{1} \times X \rightarrow X$ be the projection. We set $\mathcal{E}^{\triangle}:=p^{-1} E$ on which we have the natural connection $d_{\mathbb{P}^{1}}$ along the $\mathbb{P}^{1}$-direction. We set

$$
\nabla_{\lambda}:=d_{\mathbb{P}^{1}}+\left(\lambda^{-1} \mathcal{U}-\mathcal{Q}-\lambda \mathcal{U}^{\dagger}\right) \frac{d \lambda}{\lambda}
$$

This gives a flat connection on $\mathcal{E}^{\triangle}$ along the $\mathbb{P}^{1}$-direction. Then we obtain a $T \widetilde{T} E$ structure

$$
\widetilde{\mathbb{D}}^{\triangle}:=\left(\bar{\partial}_{E}+\lambda \theta^{\dagger}\right)+\left(\partial_{E}+\lambda^{-1} \theta\right)+\nabla_{\lambda}: \mathcal{E}^{\triangle} \rightarrow \mathcal{E}^{\triangle} \otimes \widetilde{\Omega}_{\mathbb{P}^{1} \times X}^{1}
$$

The pairing $\mathcal{S}$ is induced by $\mathcal{S}\left(u \otimes \sigma^{*} v\right)=h\left(u, \sigma^{*} v\right)$.

Let us also look at the gluing construction of the above $\left(\mathcal{E}^{\triangle}, \widetilde{\mathbb{D}}^{\triangle}, \mathcal{S}\right)$. Let $\left(E, \bar{\partial}_{E}, \theta, h, \mathcal{U}, \mathcal{Q}\right)$ be as above. Let $p_{\lambda}$ be the projection $\mathcal{X} \rightarrow X$. Let $\mathcal{E}$ be the holomorphic vector bundle $\left(p_{\lambda}^{-1} E, \bar{\partial}_{E}+\lambda \theta^{\dagger}+\bar{\partial}_{\lambda}\right)$, where $\bar{\partial}_{\lambda}$ denotes the natural $\lambda$-holomorphic structure on $\mathcal{E}$. We have the family of flat $\lambda$-connections $\mathbb{D}=\bar{\partial}_{E}+$ $\lambda \theta^{\dagger}+\lambda \partial_{E}+\theta$ on $\mathcal{E}$. The associated family of flat connections is given by $\mathbb{D}^{f}=$ $\bar{\partial}_{E}+\lambda \theta^{\dagger}+\partial_{E}+\lambda^{-1} \theta$. Then $\widetilde{\mathbb{D}}^{f}:=\mathbb{D}^{f}+\nabla_{\lambda}$ gives a meromorphic flat connection 
on $\mathcal{E}$. Let $p_{\mu}$ be the projection $\mathcal{X}^{\dagger} \rightarrow X^{\dagger}$. Let $\mathcal{E}^{\dagger}$ be the holomorphic vector bundle $\left(p_{\mu}^{-1} E, \partial_{E}+\mu \theta+\bar{\partial}_{\mu}\right)$, where $\bar{\partial}_{\mu}$ denotes the natural $\mu$-holomorphic structure on $\mathcal{E}^{\dagger}$. We have the family of flat $\mu$-connections $\mathbb{D}^{\dagger}=\partial_{E}+\mu \theta+\mu \bar{\partial}_{E}+\theta^{\dagger}$ on $\mathcal{E}^{\dagger}$. The associated family of flat connections is given by $\mathbb{D}^{\dagger} f=\partial_{E}+\mu \theta+\bar{\partial}_{E}+\mu^{-1} \theta^{\dagger}$. Then $\widetilde{\mathbb{D}}^{\dagger f}:=\mathbb{D}^{\dagger f}+\nabla_{\lambda}$ gives a meromorphic flat connection on $\mathcal{E}^{\dagger}$.

We have the induced pairings $\mathcal{S}_{0}: \mathcal{E} \otimes \sigma^{*} \mathcal{E}^{\dagger} \rightarrow \mathcal{O}_{\mathcal{X}}$ and $\mathcal{S}_{\infty}: \mathcal{E}^{\dagger} \otimes \sigma^{*} \mathcal{E} \rightarrow \mathcal{O}_{\mathcal{X}^{\dagger}}$ induced by $h$. Then $\left(\mathcal{E}^{\triangle}, \widetilde{\mathbb{D}}^{\wedge}, \mathcal{S}\right)$ is obtained as the gluing of $\left(\mathcal{E}, \widetilde{\mathbb{D}}^{f}\right),\left(\mathcal{E}^{\dagger}, \widetilde{\mathbb{D}}^{\dagger} f\right)$ and $\left(\mathcal{S}_{0}, \mathcal{S}_{\infty}\right)$ by the procedure in Subsection 2.1.6.

2.1.8. TERP and twistor-TERP. Let us check that the notions of TERP structure and twistor-TERP structure are equivalent. First, let us introduce a pairing $P$ induced by $\kappa$ and $\mathcal{S}$. Then we deduce the equivalence in the case that $X$ is a point, for simplicity. We give a remark for the family case in the end.

The induced pairing $P$. We set $j:=\gamma \circ \sigma=\sigma \circ \gamma$, which is a holomorphic involution of $\mathbb{P}^{1}$. We have the induced isomorphisms

$$
\sigma^{*} \kappa: j^{*} \mathbb{T}(w) \simeq \sigma^{*} \mathbb{T}(w), \quad j^{*} \kappa: \sigma^{*} \mathbb{T}(w) \simeq j^{*} \mathbb{T}(w)
$$

We have the following equality:

$$
\sigma^{*} \kappa \circ j^{*} \kappa=j^{*}\left(\gamma^{*} \kappa \circ \kappa\right)=j^{*}(\mathrm{id})=\mathrm{id} .
$$

We will use similar relations implicitly. We also remark the commutativity of the following diagram, which can be checked by a direct calculation:

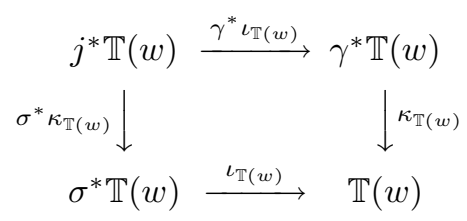

The composite $j^{*} \mathbb{T}(w) \rightarrow \mathbb{T}(w)$ is denoted by $\rho_{\mathbb{T}(w)}$.

Let $\left(V, \widetilde{\mathbb{D}}_{V}^{\triangle}, \mathcal{S}, \kappa,-w\right)$ be a variation of twistor-TERP structure. We define a pairing $P: V \otimes j^{*} V \rightarrow \mathbb{T}(-w)$ by

$$
P:=(\sqrt{-1})^{w} \cdot \mathcal{S} \circ\left(1 \otimes \sigma^{*} \kappa\right) .
$$

Lemma 2.1. $P$ is $(-1)^{w}$-symmetric in the sense that the diagram

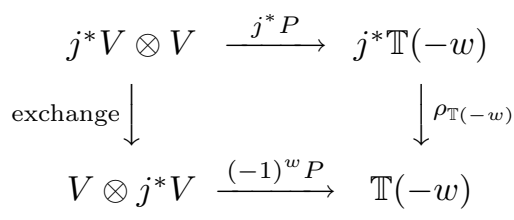


is commutative, that is, $\rho_{\mathbb{T}(-w)} \circ j^{*} P=(-1)^{w} P \circ$ exchange. Here, exchange denotes the natural morphism exchanging the components.

Proof. We have the following equalities:

$$
\begin{aligned}
\rho_{\mathbb{T}(-w)} \circ j^{*} P & =(\sqrt{-1})^{w} \kappa_{\mathbb{T}(-w)} \circ \gamma^{*} \iota_{\mathbb{T}(-w)} \circ j^{*} \mathcal{S} \circ\left(1 \otimes j^{*} \sigma^{*} \kappa\right) \\
& =(\sqrt{-1})^{w} \kappa_{\mathbb{T}(-w)} \circ \gamma^{*} \iota_{\mathbb{T}(-w)} \circ\left(\gamma^{*} \sigma^{*} \mathcal{S}\right) \circ\left(1 \otimes \gamma^{*} \kappa\right) \\
& =(\sqrt{-1})^{w} \kappa_{\mathbb{T}(-w)} \circ \gamma^{*}\left(\iota_{\mathbb{T}(-w)} \circ \sigma^{*} \mathcal{S}\right) \circ\left(1 \otimes \gamma^{*} \kappa\right) .
\end{aligned}
$$

By using the compatibility of $\mathcal{S}$ and $\kappa$, we obtain

$$
\begin{aligned}
& (-1)^{w} P \circ \text { exchange }=(\sqrt{-1})^{w}(-1)^{w} \mathcal{S} \circ\left(1 \otimes \sigma^{*} \kappa\right) \circ \text { exchange } \\
& =(\sqrt{-1})^{w}(-1)^{w} \mathcal{S} \circ\left(\kappa \otimes \sigma^{*} \kappa\right) \circ\left(\gamma^{*} \kappa \otimes 1\right) \circ \text { exchange } \\
& =(\sqrt{-1})^{w} \kappa_{\mathbb{T}(-w)} \circ \gamma^{*}\left((-1)^{w} \mathcal{S} \circ \text { exchange }\right) \circ\left(1 \otimes \gamma^{*} \kappa\right) .
\end{aligned}
$$

Thus, we are done.

Lemma 2.2. The following diagram is commutative:

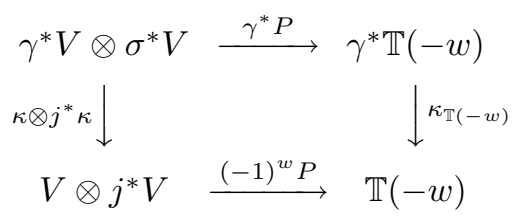

That is, $(-1)^{w} P \circ\left(\kappa \otimes j^{*} \kappa\right)=\kappa_{\mathbb{T}(-w)} \circ \gamma^{*} P$.

Proof. We have the following equalities:

$$
\begin{aligned}
(\sqrt{-1})^{-w} P \circ\left(\kappa \otimes j^{*} \kappa\right) & =\mathcal{S} \circ\left(1 \otimes \sigma^{*} \kappa\right) \circ\left(\kappa \otimes j^{*} \kappa\right) \\
& =\mathcal{S} \circ\left(\kappa \otimes \sigma^{*} \kappa\right) \circ\left(1 \otimes j^{*} \kappa\right), \\
\kappa_{\mathbb{T}(-w)} \circ \gamma^{*}\left((\sqrt{-1})^{-w} P\right) & =\kappa_{\mathbb{T}(-w)} \circ \gamma^{*}\left(\mathcal{S} \circ\left(1 \otimes \sigma^{*} \kappa\right)\right) \\
& =\kappa_{\mathbb{T}(-w)} \circ\left(\gamma^{*} \mathcal{S}\right) \circ\left(1 \otimes j^{*} \kappa\right) .
\end{aligned}
$$

Thus, the claim follows from the compatibility of $\mathcal{S}$ and $\kappa$.

From twistor-TERP to TERP. Let $(V, \nabla, \mathcal{S}, \kappa,-w)$ be a twistor-TERP structure. Let us explain how to associate a TERP structure $\left(H, H_{\mathbb{R}}^{\prime}, \nabla, P^{\prime},-w\right)$ in the sense of Hertling (Definition 3.1 in [12]), where $(H, \nabla)$ is a $T E$-structure, $H_{\mathbb{R}}^{\prime}$ is a real structure on the flat bundle $H_{\mid \mathbb{C}_{\lambda}^{*}}$, and $P^{\prime}$ is a pairing (see below). We set $H:=V_{\mid \mathbb{C}_{\lambda}}$ and $H^{\prime}:=V_{\mid \mathbb{C}_{\lambda}^{*}}$. In general, for a $\mathbb{C}$-vector bundle $U$, let $\bar{U}$ denote the conjugate of $U$, i.e., $\bar{U}=U$ as an $\mathbb{R}$-vector bundle, and multiplication of $\sqrt{-1}$ 
on $\bar{U}$ is given by multiplication of $-\sqrt{-1}$ on $U$. Note that $\gamma^{*}(H)_{\mid \lambda}$ for $\lambda \neq 0$ is naturally identified with $\bar{H}_{\mid \bar{\lambda}^{-1}}$.

The following diagram for $\lambda \neq 0$ is commutative by the flatness of $\kappa$ :

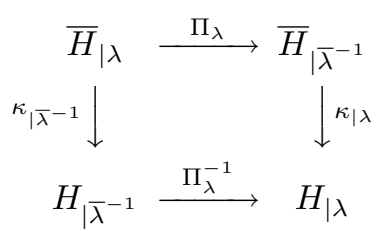

Here, $\Pi_{\lambda}$ denotes the parallel transport along the segment connecting $\lambda$ and $\bar{\lambda}^{-1}$, which is frequently used in [10]. A flat isomorphism $\kappa^{\prime}: \bar{H}_{\mid \mathbb{C}_{\lambda}^{*}} \simeq H_{\mid \mathbb{C}_{\lambda}^{*}}$ is given by the composite $\kappa_{\mid \lambda}^{\prime}:=\kappa_{\mid \lambda} \circ \Pi_{\lambda}$. Because $\gamma^{*} \kappa \circ \kappa=\mathrm{id}$, the composite

$$
\bar{H}_{\mid \lambda} \stackrel{\kappa_{\mid \bar{\lambda}^{-1}}}{\longrightarrow} H_{\mid \bar{\lambda}^{-1}} \stackrel{\kappa_{\lambda}}{\longrightarrow} \bar{H}_{\mid \lambda}
$$

is the identity. Then we can check $\kappa^{\prime} \circ \kappa^{\prime}=$ id by using the commutativity 2.10, as follows:

$$
\kappa_{\mid \lambda}^{\prime} \circ \kappa_{\mid \lambda}^{\prime}=\left(\kappa_{\mid \lambda} \circ \Pi_{\lambda}\right) \circ\left(\Pi_{\lambda}^{-1} \circ \kappa_{\mid \bar{\lambda}^{-1}}\right)=\kappa_{\mid \lambda} \circ \kappa_{\mid \bar{\lambda}^{-1}}=\mathrm{id}
$$

Hence, $\kappa^{\prime}$ gives a flat real structure on $H^{\prime}$. Thus, we obtain a real flat subbundle $H_{\mathbb{R}}^{\prime}$ of $H_{\mid \mathbb{C}_{\lambda}^{*}}$. By restricting $P$, we obtain a pairing

$$
P_{\mid \mathbb{C}_{\lambda}}: H \otimes j^{*} H \rightarrow \mathbb{T}(-w)_{\mid \mathbb{C}_{\lambda}}=\mathcal{O}_{\mathbb{C}_{\lambda}} \cdot(\sqrt{-1} \lambda)^{-w} t_{1}^{(-w)}
$$

By taking the coefficients of $t_{1}^{(-w)}$, we obtain a flat morphism

$$
P^{\prime}: H^{\prime} \otimes j^{*} H^{\prime} \rightarrow \mathcal{O}_{\mathbb{C}_{\lambda}^{*}}
$$

such that $\lambda^{w} \cdot P^{\prime}$ induces a perfect pairing $H \otimes j^{*} H \rightarrow \mathcal{O}_{\mathbb{C}_{\lambda}}$. By Lemma 2.1. $P^{\prime}$ is $(-1)^{w}$-symmetric.

Lemma 2.3. $P^{\prime}\left(H_{\mathbb{R}}^{\prime} \otimes_{\mathbb{R}} j^{*} H_{\mathbb{R}}^{\prime}\right) \subset(\sqrt{-1})^{w} \mathbb{R}$.

Proof. Note that $\kappa$ gives real structures $\kappa_{\mid a}: \overline{H_{\mid a}} \simeq H_{\mid a}$ for $a=1,-1$. By Lemma 2.2 , we have

$$
(\sqrt{-1})^{w} P_{\mid 1} \circ\left(\kappa_{\mid 1} \otimes \kappa_{\mid-1}\right)=\left(\kappa_{\mathbb{T}(-w)}\right)_{\mid 1} \circ\left((\sqrt{-1})^{w} P_{\mid 1}\right) .
$$

We obtain $P_{\mid 1}^{\prime}\left(H_{\mathbb{R} \mid 1} \otimes H_{\mathbb{R} \mid-1}\right) \subset(\sqrt{-1})^{w} \mathbb{R}$. Then the claim follows from the flatness of $P^{\prime}$.

Thus, we obtain a TERP structure $\left(H, H_{\mathbb{R}}^{\prime}, \nabla, P^{\prime},-w\right)$. 
From TERP to twistor-TERP. Conversely, we obtain a twistor-TERP structure $(V, \nabla, \kappa, \mathcal{S},-w)$ from a TERP structure $\left(H, H_{\mathbb{R}}^{\prime}, \nabla, P^{\prime},-w\right)$. The following construction has already essentially appeared in [10. We set $V_{0}:=H$ and $V_{\infty}:=$ $\gamma^{*} H$. We have the flat isomorphism

$$
\tau_{\text {real }}: H_{\mid \mathbb{C}_{\lambda}^{*}} \simeq \gamma^{*}\left(H_{\mid \mathbb{C}_{\lambda}^{*}}\right),
$$

obtained as the composite of the conjugate with respect to the real structure and the parallel transport along the segment connecting $\lambda$ and $\bar{\lambda}^{-1}$. By gluing $(H, \nabla)$ and $\gamma^{*}(H, \nabla)$ via $\tau_{\text {real }}$, we obtain an integrable twistor structure $(V, \nabla)$.

By construction, we have $\gamma^{*}\left(\tau_{\text {real }}\right)=\tau_{\text {real }}^{-1}$, and the following diagram is commutative:

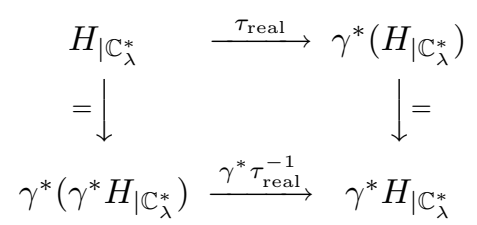

Hence, a morphism $\kappa: \gamma^{*}(V, \nabla) \simeq(V, \nabla)$ is given by the gluing of $\gamma^{*} V_{\infty} \simeq V_{0}$ and $\gamma^{*} V_{0} \simeq V_{\infty}$ induced by the identity. Clearly it satisfies $\gamma^{*} \kappa \circ \kappa=\mathrm{id}$. The restriction $\kappa_{\mid \mathbb{C}_{\lambda}^{*}}: \gamma^{*}(V)_{\mid \mathbb{C}_{\lambda}^{*}} \rightarrow V_{\mid \mathbb{C}_{\lambda}^{*}}$ is identified with $\tau_{\text {real }}^{-1}: \gamma^{*} H_{\mid \mathbb{C}_{\lambda}^{*}} \simeq H_{\mid \mathbb{C}_{\lambda}^{*}}$.

Let $P_{0}: V_{0} \otimes j^{*} V_{0} \rightarrow \mathcal{O}_{\mathbb{C}_{\lambda}} \cdot t_{0}^{(-w)}$ be given by

$$
P_{0}=P^{\prime} \cdot t_{1}^{(-w)}=P^{\prime} \cdot(\sqrt{-1} \lambda)^{w} \cdot t_{0}^{(-w)} .
$$

We have the induced morphism

$$
\kappa_{\mathbb{T}(-w)} \circ \gamma^{*} P_{0}: V_{\infty} \otimes j^{*} V_{\infty} \rightarrow \mathcal{O}_{\mathbb{C}_{\mu}} \cdot t_{\infty}^{(-w)} .
$$

Because $P^{\prime}\left(H_{\mathbb{R}}^{\prime} \otimes_{\mathbb{R}} j^{*} H_{\mathbb{R}}^{\prime}\right) \subset(\sqrt{-1})^{w} \mathbb{R}$, we obtain the following equalities for linear maps $\overline{H_{\mid 1}} \otimes \overline{H_{\mid-1}} \rightarrow \mathbb{T}(-w)_{\mid 1}$ :

$$
\begin{aligned}
(\sqrt{-1})^{w} P_{0 \mid 1} \circ\left(\kappa_{\mid 1} \otimes \kappa_{\mid-1}\right) & =\left(\kappa_{\mathbb{T}(-w)}\right)_{\mid 1} \circ\left((\sqrt{-1})^{w} P_{0 \mid 1}\right) \\
& =(-\sqrt{-1})^{w}\left(\kappa_{\mathbb{T}(-w)}\right)_{\mid 1} \circ\left(\gamma^{*} P_{0 \mid 1}\right) .
\end{aligned}
$$

Here, we have used the natural identification $P_{0 \mid 1}=\left(\gamma^{*} P_{0}\right)_{\mid 1}$. The first and third terms in 2.12) are obtained as the restrictions of morphisms $\left(V_{\infty} \otimes j^{*} V_{\infty}\right)_{\mid \mathbb{C}_{\lambda}^{*}} \rightarrow$ $\mathcal{O}_{\mathbb{C}_{\lambda}^{*}} \cdot t_{1}^{(-w)}$ to the fiber over 1 . By flatness, we obtain the following equality on $\mathbb{C}_{\lambda}^{*}$ :

$$
(-1)^{w} P_{0} \circ\left(\kappa \otimes j^{*} \kappa\right)=\kappa_{\mathbb{T}(-w)} \circ \gamma^{*} P_{0} .
$$

Hence, the pairings $P_{0}$ and $(-1)^{w} \kappa_{\mathbb{T}(-w)} \circ \gamma^{*} P_{0}$ induce $P: V \otimes j^{*} V \rightarrow \mathbb{T}(-w)$. Since $P^{\prime}$ is $(-1)^{w}$-symmetric, $P$ is also $(-1)^{w}$-symmetric in the sense of Lemma 2.1 . 
From 2.13, we obtain

$$
(-1)^{w} P \circ\left(\kappa \otimes j^{*} \kappa\right)=\kappa_{\mathbb{T}(-w)} \circ \gamma^{*} P .
$$

The pairing $\mathcal{S}$ is constructed from $P$ and $\kappa$ by the relation 2.5). The compatibility of $\kappa$ and $\mathcal{S}$ follows from $(2.8), 2.9$ and 2.14 . The pairing $\mathcal{S}$ is $(-1)^{w}$-symmetric, which follows from $2.6,2.7$ and the compatibility with $\kappa$. Thus, we obtain a twistor-TERP structure $(V, \nabla, \mathcal{S}, \kappa,-w)$.

Hertling's vector bundle. Let $\left(H, H_{\mathbb{R}}^{\prime}, \nabla, P,-w\right)$ be a TERP structure corresponding to a twistor-TERP structure $(V, \nabla, \mathcal{S}, \kappa,-w)$. Recall that Hertling constructed an integrable twistor structure $(\widehat{H}, \nabla)$ from a TERP structure $\left(H, H_{\mathbb{R}}^{\prime}, \nabla, P,-w\right)$ by gluing $H$ and $\gamma^{*} H$ via a map $\tau$. (See [10].) We do not recall $\tau$ and his construction here, but $\widehat{H}$ is naturally isomorphic to $V \otimes \mathcal{O}(0,-w)$ by the following correspondence:

$$
\begin{gathered}
H=V_{0} \leftrightarrow V_{0} \otimes \mathcal{O}(0,-w)_{0}, \quad a \leftrightarrow a \otimes f_{0}^{(0,-w)}, \\
\gamma^{*} H \leftrightarrow \gamma^{*} V_{0} \otimes \mathcal{O}(0,-w)_{\infty}, \quad \gamma^{*} b \leftrightarrow \gamma^{*} b \otimes(\sqrt{-1})^{w} f_{\infty}^{(0,-w)} .
\end{gathered}
$$

According to 10 and $12,\left(H, H_{\mathbb{R}}^{\prime}, \nabla, P,-w\right)$ is defined to be pure if $(\widehat{H}, \nabla)$ is pure of weight 0 . They consider the hermitian pairing $h$ on $H^{0}\left(\mathbb{P}^{1}, \widehat{H}\right)$ given by $\lambda^{w} \cdot P^{\prime} \circ(1 \otimes \tau)$, and $\left(H, H_{\mathbb{R}}^{\prime}, \nabla, P,-w\right)$ is defined to be polarized if $h$ is positive definite.

Lemma 2.4. $\left(H, H_{\mathbb{R}}^{\prime}, \nabla, P,-w\right)$ is pure (resp. polarized) if and only if the corresponding $(V, \nabla, \mathcal{S}, \kappa,-w)$ is pure (resp. polarized).

Proof. The purity claim is obvious. Let us consider polarizability. We have only to show that $h$ is the hermitian pairing induced by $\widetilde{\mathcal{S}}:=\mathcal{S} \otimes \mathcal{S}_{0,-w}$, under the identification of $\widehat{H}$ and $V \otimes \mathcal{O}(0,-w)$.

Let $\widehat{a}, \widehat{b} \in H^{0}\left(\mathbb{P}^{1}, \widehat{H}\right)$. Under the identification $\widehat{H}_{\mid \mathbb{C}_{\lambda}}=H$ and $\widehat{H}_{\mid \mathbb{C}_{\mu}}=\gamma^{*} H$, the sections $a$ and $b$ of $H$ are determined by $a:=\widehat{a}_{\mid \mathbb{C}_{\lambda}}$ and $\gamma^{*} b:=\widehat{b}_{\mid \mathbb{C}_{\mu}}$. By definition,

$$
h(\widehat{a}, \widehat{b})=\lambda^{w} P^{\prime}\left(a, j^{*} b\right) .
$$

Let us look at $\widetilde{\mathcal{S}}_{\mid \mathbb{C}_{\lambda}}$. Under the above identification, the pairing of $\widehat{a}$ and $\widehat{b}$ is given by

$$
\begin{aligned}
\widetilde{\mathcal{S}}\left(a \otimes f_{0}^{(0,-w)}, \sigma^{*}\left(\gamma^{*} b \otimes(\sqrt{-1})^{w} f_{\infty}^{(0,-w)}\right)\right) & =\mathcal{S}\left(a, \sigma^{*}\left(\gamma^{*} b\right)\right) \cdot t_{0}^{(w)} \\
& =\mathcal{S}\left(a, j^{*} b\right) \cdot t_{0}^{(w)}=: \mathcal{S}_{0}\left(a, j^{*} b\right) .
\end{aligned}
$$


Let us compare $\lambda^{w} P^{\prime}\left(a, j^{*} b\right)$ and $\mathcal{S}_{0}\left(a, j^{*} b\right)$. Since $\kappa_{\mid \mathbb{C}_{\mu}}$ is equal to the identity $V_{\infty}=\gamma^{*} H \rightarrow \gamma^{*} V_{0}=\gamma^{*} H$, we have

$$
P_{\mid \mathbb{C}_{\lambda}}=(\sqrt{-1})^{w} \mathcal{S} \circ\left(1 \otimes \sigma^{*} \kappa\right)_{\mid \mathbb{C}_{\lambda}}=(\sqrt{-1})^{w} \mathcal{S}_{\mid \mathbb{C}_{\lambda}}
$$

Hence,

$$
\begin{aligned}
P^{\prime}\left(a, j^{*} b\right) \cdot t_{1}^{(-w)} & =P\left(a, j^{*} b\right)=\mathcal{S}\left(a, j^{*} b\right) \cdot(\sqrt{-1})^{w} \\
& =(\sqrt{-1})^{w} \cdot \mathcal{S}_{0}\left(a, j^{*} b\right) \cdot t_{0}^{(-w)}=\lambda^{-w} \mathcal{S}_{0}\left(a, j^{*} b\right) \cdot t_{1}^{(-w)}
\end{aligned}
$$

Thus, we obtain $\lambda^{w} \cdot P^{\prime}\left(a, j^{*} b\right)=\mathcal{S}_{0}\left(a, j^{*} b\right)$. Therefore, $\widetilde{\mathcal{S}}$ induces $h$.

Family version. The correspondence is generalized in the family case. Let $\left(V, \widetilde{\mathbb{D}}_{V}^{\triangle}, \mathcal{S}, \kappa,-w\right)$ be a variation of twistor-TERP structure. We set $H:=V_{\mid \mathbb{C}_{\lambda} \times X}$. It is equipped with $T E$-structure $\nabla$ obtained as the restriction of $\widetilde{\mathbb{D}}_{V}^{\Delta}$. As in the previous case, we obtain a flat $\mathbb{C}$-anti-linear isomorphism $\kappa^{\prime}: H_{\mid \mathbb{C}_{\lambda}^{*} \times X} \simeq H_{\mid \mathbb{C}_{\lambda}^{*} \times X}$ and a flat pairing $P: H^{\prime} \otimes j^{*} H^{\prime} \rightarrow \mathcal{O}_{\mathbb{C}_{\lambda}^{*} \times X}$. It is easy to check that $\left(H, H_{\mathbb{R}}^{\prime}, \nabla, P,-w\right)$ is a variation of TERP structure. The converse can be constructed similarly. The correspondence preserves "pure" and "polarized", for which we have only to check the case in which $X$ is a point (Lemma 2.4).

\section{§2.2. Basic examples}

2.2.1. Example associated to a holomorphic function. Let $\mathfrak{a}$ be a holomorphic function on a complex manifold $X$. We set

$$
\begin{aligned}
V_{0} & :=\mathcal{O}_{\mathbb{C}_{\lambda} \times X} \cdot e, & \nabla_{V_{0}}(e) & =e \cdot d\left(\lambda^{-1} \mathfrak{a}\right), \\
V_{\infty} & :=\mathcal{O}_{\mathbb{C}_{\mu} \times X^{\dagger}} \cdot e^{\dagger}, & \nabla_{V_{\infty}}\left(e^{\dagger}\right) & =e^{\dagger} \cdot d\left(\mu^{-1} \overline{\mathfrak{a}}\right) .
\end{aligned}
$$

We put $s:=\exp \left(-\lambda^{-1} \mathfrak{a}\right) \cdot e$ and $s^{\dagger}:=\exp \left(-\mu^{-1} \overline{\mathfrak{a}}\right) \cdot e^{\dagger}$, which are flat sections of $V_{0 \mid \mathbb{C}_{\lambda}^{*} \times X}$ and $V_{\infty \mid \mathbb{C}_{\mu}^{*} \times X^{\dagger}}$, respectively. A gluing $\Phi: V_{0 \mid \mathbb{C}_{\lambda}^{*} \times X} \simeq V_{\infty \mid \mathbb{C}_{\mu}^{*} \times X^{\dagger}}$ is given by $\Phi(s)=s^{\dagger}$, in other words,

$$
\Phi(e)=\exp \left(\lambda^{-1} \mathfrak{a}-\mu^{-1} \overline{\mathfrak{a}}\right) \cdot e^{\dagger} .
$$

Let $V$ be the $C^{\infty}$-bundle obtained as the gluing of $V_{0}$ and $V_{\infty}$ via $\Phi$, which is equipped with a $T \widetilde{T} E$-structure. For each point $P \in X$, the restriction $V_{\mid \mathbb{P}^{1} \times\{P\}}$ is isomorphic to $\mathcal{O}_{\mathbb{P}^{1}}$, and hence $\left(V, \widetilde{\mathbb{D}}_{V}^{\triangle}\right)$ is pure of weight 0 . A real structure $\kappa$ is given by $\kappa\left(\gamma^{*} e^{\dagger}\right)=e$ and $\kappa\left(\gamma^{*} e\right)=e^{\dagger}$. We can check that $\kappa$ actually gives a flat isomorphism $\gamma^{*} V \simeq V$. A pairing $\mathcal{S}$ of $V$ with weight 0 is given by $e \otimes \sigma^{*} e^{\dagger} \mapsto t_{0}^{(0)}$ and $e^{\dagger} \otimes \sigma^{*} e \mapsto t_{\infty}^{(0)}$. It is easy to check that $\mathcal{S}$ actually gives a symmetric flat pairing $V \otimes \sigma^{*} V \rightarrow \mathbb{T}(0)_{X}$. The compatibility of $\mathcal{S}$ and $\kappa$ can be checked by a 
direct calculation:

$$
\begin{gathered}
\kappa_{\mathbb{T}(0)} \circ \gamma^{*} \mathcal{S}\left(\gamma^{*} e^{\dagger} \otimes \gamma^{*} \sigma^{*} e\right)=\kappa_{\mathbb{T}(0)}\left(\gamma^{*}\left(\mathcal{S}\left(e^{\dagger} \otimes \sigma^{*} e\right)\right)\right)=\kappa_{\mathbb{T}(0)} \gamma^{*} t_{\infty}^{(0)}=t_{0}^{(0)}, \\
\mathcal{S} \circ\left(\kappa \otimes \sigma^{*} \kappa\right)\left(\gamma^{*} e^{\dagger} \otimes \sigma^{*} \gamma^{*} e\right)=\mathcal{S}\left(e \otimes \sigma^{*} e^{\dagger}\right)=t_{0}^{(0)} .
\end{gathered}
$$

Hence, we obtain a variation of twistor-TERP structure denoted by $L(\mathfrak{a})$. It is polarized. The underlying harmonic bundle is given by the line bundle $\mathcal{O}_{X} \cdot v$ with the Higgs field $\theta \cdot v=v \cdot d \mathfrak{a}$ and the hermitian metric $h(v, v)=1$, where $v:=e_{\mid\{0\} \times X}$. The operators $\mathcal{U}$ and $\mathcal{Q}$ are given by $\mathcal{U}=-\mathfrak{a}$ and $\mathcal{Q}=0$.

2.2.2. Example associated to unitary flat bundles of rank one. In general, a variation of pure polarized Hodge structure provides us with an example of a variation of pure polarized integrable twistor structure. Any unitary flat bundle naturally gives a variation of pure polarized Hodge structure, and hence a variation of pure polarized integrable twistor structure.

In particular, we will use the following example. Let $X:=\mathbb{C}^{n}$ and $D:=$ $\bigcup_{i=1}^{\ell}\left\{z_{i}=0\right\}$. For any $\boldsymbol{a} \in \mathbb{R}^{\ell}$, we have the following unitary flat bundle:

$$
\mathcal{O}_{X-D} \cdot e, \quad \nabla e=e \cdot\left(-\sum_{i=1}^{\ell} a_{i} \frac{d z_{i}}{z_{i}}\right) .
$$

The associated variation of integrable polarized pure integrable structure is denoted by $L(\boldsymbol{a})$.

More concretely, it is obtained as the gluing of the following meromorphic flat bundles:

$$
\begin{array}{rrr}
V_{0}=\mathcal{O}_{\mathbb{C}_{\lambda} \times(X-D)} \cdot e, & \nabla_{V_{0}} e=e \cdot\left(-\sum_{i=1}^{\ell} a_{i} \frac{d z_{i}}{z_{i}}\right), \\
V_{\infty}=\mathcal{O}_{\mathbb{C}_{\mu} \times\left(X^{\dagger}-D^{\dagger}\right)} \cdot e^{\dagger}, & \nabla_{V_{\infty}} e^{\dagger}=e^{\dagger} \cdot\left(\sum_{i=1}^{\ell} a_{i} \frac{d \bar{z}_{i}}{\bar{z}_{i}}\right) .
\end{array}
$$

The gluing is given by $\Phi(e)=\prod_{i=1}^{\ell}\left|z_{i}\right|^{-2 a_{i}} \cdot e^{\dagger}$. The pairing is given by $\mathcal{S}\left(e, \sigma^{*} e^{\dagger}\right)$ $=1$. The underlying harmonic bundle is the line bundle $\mathcal{O}_{X-D} \cdot v$ with the Higgs field $\theta \cdot v=0$ and the metric $h(v, v)=\prod_{i=1}^{\ell}\left|z_{i}\right|^{-2 a_{i}}$, where $v:=e_{\mid\{0\} \times(X-D)}$. The operators $\mathcal{U}$ and $\mathcal{Q}$ are 0 .

2.2.3. Example induced by nilpotent maps. Let $Y$ be a complex manifold. We set $X:=\mathbb{C}^{\ell} \times Y, D=\bigcup_{i=1}^{\ell}\left\{z_{i}=0\right\} \times Y$. We put $\mathcal{X}:=\mathbb{C}_{\lambda} \times X$ and $\mathcal{X}^{\dagger}:=\mathbb{C}_{\mu} \times X^{\dagger}$. We use the symbols $\mathcal{D}, \mathcal{Y}, \mathcal{D}^{\dagger}$ and $\mathcal{Y}^{\dagger}$ in similar meanings. Let $q_{0}: \mathcal{X} \rightarrow \mathcal{Y}$ and $q_{\infty}: \mathcal{X}^{\dagger} \rightarrow \mathcal{Y}^{\dagger}$ denote the naturally defined projections. 
Let $\left(V, \mathbb{D}^{\triangle}\right)$ be a variation of $\mathbb{P}^{1}$-holomorphic vector bundle on $\mathbb{P}^{1} \times Y$ with a tuple $\boldsymbol{f}$ of nilpotent morphisms

$$
f_{i}: V \rightarrow V \otimes \mathbb{T}(-1), \quad i=1, \ldots, \ell .
$$

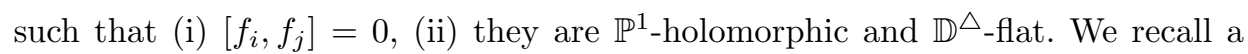
construction of the variation of $\mathbb{P}^{1}$-holomorphic vector bundle on $\mathbb{P}^{1} \times(X-D)$ associated to $(V, \boldsymbol{f})$ given in Subsection 3.5.3 of [22] with a minor generalization. (In 22, we considered the case that $Y$ is a point.)

We regard $\left(V, \mathbb{D}_{V}^{\triangle}\right)$ as the gluing of a family of $\lambda$-flat bundles $\left(V_{0}, \mathbb{D}_{V_{0}}\right)$ on $\mathcal{Y}$, and a family of $\mu$-flat bundles $\left(V_{\infty}, \mathbb{D}_{V_{\infty}}^{\dagger}\right)$ on $\mathcal{Y}^{\dagger}$. We obtain a holomorphic vector bundle $\mathcal{V}_{0}:=q_{0}^{*} V_{0}$ on $\mathcal{X}-\mathcal{D}$ with a family of flat $\lambda$-connections $q_{0}^{*} \mathbb{D}_{V_{0}}$. We naturally identify $\mathbb{T}(0)_{\mid \mathcal{X}-\mathcal{D}} \simeq \mathcal{O}_{\mathcal{X}-\mathcal{D}}$ by the trivialization $t_{0}^{(0)}$. We also use the natural identification $\mathbb{T}(-1) \otimes \mathbb{T}(1) \simeq \mathbb{T}(0)$. We have the $q_{0}^{*} \mathbb{D}_{V_{0}}$-flat endomorphisms $q_{0}^{*} f_{i} \otimes$ $t_{0}^{(1)} \in \operatorname{End}\left(\mathcal{V}_{0}\right)$. We obtain the family of flat $\lambda$-connections on $\mathcal{V}_{0}$ given as follows:

$$
\mathbb{D}_{\mathcal{V}_{0}}:=q_{0}^{*} \mathbb{D}_{V_{0}}+\sum_{i=1}^{\ell} q_{0}^{*} f_{i} \otimes t_{0}^{(1)} \frac{d z_{i}}{z_{i}} .
$$

Similarly, we obtain a holomorphic vector bundle $\mathcal{V}_{\infty}:=q_{\infty}^{*} V_{\infty}$ on $\mathcal{X}^{\dagger}-\mathcal{D}^{\dagger}$ with a family of flat $\mu$-connections $q_{\infty}^{*} \mathbb{D}_{V_{\infty}}^{\dagger}$. We have the $q_{\infty}^{*} \mathbb{D}_{V_{\infty}}^{\dagger}$-flat endomorphisms $q_{\infty}^{*} f_{i} \otimes t_{\infty}^{(1)} \in \operatorname{End}\left(\mathcal{V}_{\infty}\right)$. Hence, we obtain the following family of flat $\mu$-connections:

$$
\mathbb{D}_{\mathcal{V}_{\infty}}^{\dagger}:=q_{\infty}^{*} \mathbb{D}_{V_{\infty}}^{\dagger}+\sum_{i=1}^{\ell} q_{\infty}^{*} f_{i} \otimes t_{\infty}^{(1)} \frac{d \bar{z}_{i}}{\bar{z}_{i}} .
$$

Let $\Psi_{V}: V_{0 \mid \mathbb{C}_{\lambda}^{*} \times Y} \simeq V_{\infty \mid \mathbb{C}_{\mu}^{*} \times Y}$ denote the gluing. Then an isomorphism $\Psi$ : $\mathcal{V}_{0 \mid \mathbb{C}_{\lambda}^{*} \times(X-D)} \rightarrow \mathcal{V}_{\infty \mid \mathbb{C}_{\mu}^{*} \times\left(X^{\dagger}-D^{\dagger}\right)}$ is given as follows:

$$
\Psi:=\Psi_{V} \circ \exp \left(\sum_{i=1}^{\ell} \log \left|z_{i}\right|^{2} \cdot q_{0}^{*} f_{i} \otimes \sqrt{-1} t_{1}^{(1)}\right)
$$

By construction, $\Psi$ is holomorphic with respect to $\lambda$.

Lemma 2.5. $\Psi \circ \mathbb{D}_{\mathcal{V}_{0}}^{f}=\mathbb{D}_{\mathcal{V}_{\infty}^{\dagger}}^{\dagger f} \circ \Psi$.

Proof. We have the following expressions:

$$
\begin{aligned}
\mathbb{D}_{\mathcal{V}_{0}}^{f} & =q_{0}^{*} \mathbb{D}_{V_{0}}^{f}+\sum_{i=1}^{\ell} q_{0}^{*} f_{i} \otimes\left(\sqrt{-1} t_{1}^{(1)}\right) \frac{d z_{i}}{z_{i}} \\
\mathbb{D}_{\mathcal{V}_{\infty}}^{\dagger f} & =q_{\infty}^{*} \mathbb{D}_{V_{\infty}}^{\dagger f}+\sum_{i=1}^{\ell} q_{\infty}^{*} f_{i} \otimes\left(-\sqrt{-1} t_{1}^{(1)}\right) \frac{d \bar{z}_{i}}{\bar{z}_{i}} .
\end{aligned}
$$


Because $\Psi_{V} \circ \mathbb{D}_{V_{0}}^{f}=\mathbb{D}_{V_{\infty}}^{\dagger f} \circ \Psi_{V}$, we have

$$
\begin{aligned}
q_{\infty}^{*} \mathbb{D}_{V_{\infty}}^{\dagger f} \circ \Psi-\Psi \circ q_{0}^{*} \mathbb{D}_{V_{0}}^{f} & =\Psi_{V} \circ q_{0}^{*} \mathbb{D}_{V_{0}}^{f}\left(\exp \left(\sum_{i=1}^{\ell} \log \left|z_{i}\right|^{2} \cdot q_{0}^{*} f_{i} \otimes \sqrt{-1} t_{1}^{(1)}\right)\right) \\
& =\Psi \circ\left(\sum_{i=1}^{\ell}\left(\frac{d z_{i}}{z_{i}}+\frac{d \bar{z}_{i}}{\bar{z}_{i}}\right) \cdot q_{0}^{*} f_{i} \otimes \sqrt{-1} t_{1}^{(1)}\right) .
\end{aligned}
$$

Thus, the claim of the lemma follows.

Let $\operatorname{TNIL}\left(V, \mathbb{D}_{V}^{\Delta}, \boldsymbol{f}\right)$ denote the variation of $\mathbb{P}^{1}$-holomorphic bundle on $\mathbb{P}^{1} \times$ $(X-D)$ obtained as the gluing of $\left(\mathcal{V}_{0}, \mathbb{D}_{\mathcal{V}_{0}}\right)$ and $\left(\mathcal{V}_{\infty}, \mathbb{D}_{\mathcal{V}_{\infty}}^{\dagger}\right)$ via $\Psi$.

Assume $\left(V, \mathbb{D}_{V}^{\triangle}\right)$ is equipped with a pairing $\mathcal{S}:\left(V, \mathbb{D}_{V}^{\triangle}\right) \otimes \sigma^{*}\left(V, \mathbb{D}_{V}^{\triangle}\right) \rightarrow \mathbb{T}(-w)$ of weight $w$ such that $\mathcal{S}\left(f_{i} \otimes \mathrm{id}\right)+\mathcal{S}\left(\mathrm{id} \otimes \sigma^{*} f_{i}\right)=0$ for any $i$. Then we have the induced pairing of weight $w$ :

$$
\operatorname{TNIL}(\mathcal{S}): \operatorname{TNIL}\left(V, \mathbb{D}_{V}^{\triangle}, \boldsymbol{f}\right) \otimes \sigma^{*} \operatorname{TNIL}\left(V, \mathbb{D}_{V}^{\triangle}, \boldsymbol{f}\right) \rightarrow \mathbb{T}(-w) .
$$

It is obtained as the gluing of the pairings

$$
\mathcal{S}_{0}: \mathcal{V}_{0} \otimes \sigma^{*} \mathcal{V}_{\infty} \rightarrow \mathbb{T}(-w)_{\mid \mathcal{X}-\mathcal{D}}, \quad \mathcal{S}_{\infty}: \mathcal{V}_{\infty} \otimes \sigma^{*} \mathcal{V}_{0} \rightarrow \mathbb{T}(-w)_{\mid \mathcal{X}^{\dagger}-\mathcal{D}^{\dagger}},
$$

which are the pull backs of $V_{0} \otimes \sigma^{*} V_{\infty} \rightarrow \mathbb{T}(-w)_{\mid \mathbb{C}_{\lambda}}$ and $V_{\infty} \otimes \sigma^{*} V_{0} \rightarrow \mathbb{T}(-w)_{\mid \mathbb{C}_{\mu}}$. (See Subsection 3.6.1 of [22.)

Enrichment. Assume that $\left(V, \mathbb{D}_{V}^{\triangle}\right)$ is enriched to a variation of integrable twistor structure $\left(V, \widetilde{\mathbb{D}}_{V}^{\triangle}\right)$ such that $f_{j}$ are $\widetilde{\mathbb{D}}_{V}^{\Delta}$-flat, which is obtained as the gluing of $\left(V_{0}, \nabla_{V_{0}}\right)$ and $\left(V_{\infty}, \nabla_{V_{\infty}}\right)$ via $\Psi_{V}$. Then $\operatorname{TNIL}\left(V, \mathbb{D}_{V}^{\Delta}, \boldsymbol{f}\right)$ is also enriched to integrable $\operatorname{TNIL}\left(V, \widetilde{\mathbb{D}}_{V}^{\triangle}, \boldsymbol{f}\right)$, which can be checked by an obvious enhancement of the argument in the proof of Lemma 2.5. The $T E$-structure $\nabla_{\mathcal{V}_{0}}$ and the $\widetilde{T} E$ structure $\nabla_{\mathcal{V}_{\infty}}$ are given by essentially the same formulas as 2.17):

$$
\begin{aligned}
& \nabla_{\mathcal{V}_{0}}:=q_{0}^{*} \nabla_{V_{0}}+\sum_{i=1}^{\ell} q_{0}^{*} f_{i} \otimes\left(\sqrt{-1} t_{1}^{(1)}\right) \frac{d z_{i}}{z_{i}} \\
& \nabla_{\mathcal{V}_{\infty}}:=q_{\infty}^{*} \nabla_{V_{\infty}}+\sum_{i=1}^{\ell} q_{\infty}^{*} f_{i} \otimes\left(-\sqrt{-1} t_{1}^{(1)}\right) \frac{d \bar{z}_{i}}{\bar{z}_{i}} .
\end{aligned}
$$

If we are given a pairing $\mathcal{S}$ of $\left(V, \widetilde{\mathbb{D}}_{V}^{\Delta}\right)$ with weight $w$ satisfying $\mathcal{S} \circ\left(f_{j} \otimes\right.$ id $)+\mathcal{S} \circ$ $\left(\right.$ id $\left.\otimes \sigma^{*} f_{j}\right)=0$, we have a naturally induced pairing $\operatorname{TNIL}(\mathcal{S})$ on $\operatorname{TNIL}\left(V, \widetilde{\mathbb{D}}_{V}^{\triangle}, \boldsymbol{f}\right)$ with weight $w$. Assume that we are given a real structure $\kappa$ on $\left(V, \widetilde{\mathbb{D}}_{V}^{\triangle}, \mathcal{S}\right)$ such that $\kappa \circ \gamma^{*} f_{i}=f_{i} \circ \kappa$. Because $\kappa_{0} \circ \gamma^{*}\left(f_{i} \otimes t_{1}^{(1)}\right)=\left(f_{i} \otimes t_{1}^{(1)}\right) \circ \kappa_{0}$, we obtain the isomorphisms

$$
\kappa_{0}: \gamma^{*}\left(\mathcal{V}_{\infty}, \nabla \mathcal{V}_{\infty}\right) \simeq\left(\mathcal{V}_{0}, \nabla_{\mathcal{V}_{0}}\right), \quad \kappa_{\infty}: \gamma^{*}\left(\mathcal{V}_{0}, \nabla_{\mathcal{V}_{0}}\right) \simeq\left(\mathcal{V}_{\infty}, \nabla_{\mathcal{V}_{0}}\right)
$$


We can observe that the following diagram on $\mathbb{C}_{\lambda}^{*} \times(X-D)$ is commutative:

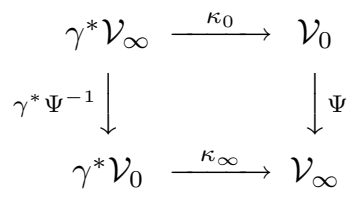

To check this, we have only to note that

$$
\begin{aligned}
& \Psi \circ \kappa=\Psi_{V} \circ \exp \left(\sum_{i=1}^{n} \log \left|z_{i}(P)\right|^{2} \cdot f_{i} \otimes \sqrt{-1} t_{1}^{(1)}\right) \circ \kappa \\
& =\kappa \circ \gamma^{*} \Psi_{V}^{-1} \circ \exp \left(-\sum_{i=1}^{n} \log \left|z_{i}(P)\right|^{2} \cdot \gamma^{*}\left(f_{i} \otimes \sqrt{-1} t_{1}^{(1)}\right)\right)=\kappa \circ \gamma^{*} \Psi^{-1} .
\end{aligned}
$$

Hence, we obtain an isomorphism

$$
\operatorname{TNIL}(\kappa): \gamma^{*} \operatorname{TNIL}\left(V, \widetilde{\mathbb{D}}_{V}^{\Delta}, \boldsymbol{f}\right) \simeq \operatorname{TNIL}\left(V, \widetilde{\mathbb{D}}_{V}^{\Delta}, \boldsymbol{f}\right) .
$$

By construction, it is easy to check $\gamma^{*} \operatorname{TNIL}(\kappa) \circ \operatorname{TNIL}(\kappa)=$ id. It is also easy to check the compatibility condition if the given $\mathcal{S}$ and $\kappa$ are compatible. Therefore, we obtain a variation of twistor-TERP structure $\operatorname{TNIL}\left(V, \widetilde{\mathbb{D}}_{V}^{\triangle}, \boldsymbol{f}, \mathcal{S}, \kappa,-w\right)$ on $X-D$ from a variation of twistor-TERP structure $\left(V, \widetilde{\mathbb{D}}_{V}^{\triangle}, \mathcal{S}, \kappa,-w\right)$ with $\boldsymbol{f}=\left(f_{i}\right)$ as above.

Definition 2.6. Let $\left(V, \mathbb{D}_{V}^{\triangle}, \boldsymbol{f}, \mathcal{S}\right)$ be as above. We set

$$
X^{*}(R):=Y \times\left\{\left(z_{1}, \ldots, z_{n}\right)|0<| z_{i} \mid<R\right\}
$$

for $R>0$.

- If there exists $R>0$ such that $\operatorname{TNIL}\left(V, \mathbb{D}_{V}^{\triangle}, \boldsymbol{f}, \mathcal{S}\right)_{\mid \mathbb{P}^{1} \times X^{*}(R)}$ is pure and polarized, it is called a twistor nilpotent orbit of weight $w$.

- If moreover $\left(V, \mathbb{D}_{V}^{\triangle}\right)$ is enriched to a variation of integrable twistor structure $\left(V, \widetilde{\mathbb{D}}_{V}^{\triangle}\right)$ such that $f_{j}$ and $\mathcal{S}$ are $\widetilde{\mathbb{D}}_{V}^{\triangle}$-flat, then $\operatorname{TNIL}\left(V, \widetilde{\mathbb{D}}_{V}^{\triangle}, \boldsymbol{f}, \mathcal{S}\right)_{\mid \mathbb{P}^{1} \times X^{*}(R)}$ is called an integrable twistor nilpotent orbit of weight $w$. (We often omit "integrable" if there is no risk of confusion.)

- If moreover $\left(V, \widetilde{\mathbb{D}}_{V}^{\triangle}, \mathcal{S}\right)$ is equipped with a real structure $\kappa$ such that $\kappa \circ \gamma^{*} f_{i}=$ $f_{i} \circ \kappa$, the variation $\operatorname{TNIL}\left(V, \widetilde{\mathbb{D}}_{V}^{\triangle}, \mathcal{S}, \kappa,-w\right)_{\mid \mathbb{P}^{1} \times X^{*}(R)}$ is called a twistor-TERP nilpotent orbit.

Remark 7. The notion of a twistor-TERP nilpotent orbit is different from "nilpotent orbit" defined by Hertling and Sevenheck. Their "nilpotent orbit" is called HS-orbit in this paper. 


\section{$\S 2.3$. Convergence}

2.3.1. Complement on convergence of pure polarized twistor structures. Let $\left(V^{(i)}, \mathcal{S}^{(i)}\right)(i=0,1)$ be polarized pure twistor structures with weight 0 of rank $r$. Let $h^{(i)}$ be the hermitian metrics on $V^{(i)}$ corresponding to $\mathcal{S}^{(i)}$, and let $d^{(i)}$ denote the associated flat unitary connections of $V^{(i)}$, which are equal to the natural connection given by holomorphic trivializations $V^{(i)} \simeq \mathcal{O}_{\mathbb{P 1}}^{\oplus r}$. Let $\bar{\partial}^{(i)}$ denote the $(0,1)$-part of $d^{(i)}$, which is equal to the holomorphic structures of $V^{(i)}$. We fix a hermitian metric $g$ of $\Omega_{\mathbb{P}^{1}}^{1,0} \oplus \Omega_{\mathbb{P}^{1}}^{0,1}$. Let $|\cdot|_{h^{(i)}, g}$ denote the sup-norms with respect to the metrics induced by $h^{(i)}$ and $g$. The symbols $|\cdot|_{h^{(i)}}$ and $|\cdot|_{g}$ are used in similar meanings. Let $\Phi: V^{(0)} \rightarrow V^{(1)}$ be a $C^{\infty}$-isomorphism such that the following conditions are satisfied for some $\epsilon>0$ :

(A1) $\left|\Phi^{*} \bar{\partial}^{(1)}-\bar{\partial}^{(0)}\right|_{h^{(0)}, g} \leq \epsilon$ as a $C^{\infty}$-section of $\operatorname{End}\left(V^{(0)}\right) \otimes \Omega^{0,1}$.

(A2) $\left|\Phi^{*} \mathcal{S}^{(1)}-\mathcal{S}^{(0)}\right|_{h^{(0)}} \leq \epsilon$ as a $C^{\infty}$-section of $\operatorname{Hom}\left(V^{(0)} \otimes \sigma^{*} V^{(0)}, \mathbb{T}(0)\right)$.

(A3) $\left|\bar{\partial}^{(0)}\left(\Phi^{*} \mathcal{S}^{(1)}-\mathcal{S}^{(0)}\right)\right|_{h^{(0)}, g}=\left|\bar{\partial}^{(0)} \Phi^{*} \mathcal{S}^{(1)}\right|_{h^{(0)}, g} \leq \epsilon$ as a $C^{\infty}$-section of $\operatorname{Hom}\left(V^{(0)} \otimes \sigma^{*} V^{(0)}, \mathbb{T}(0)\right) \otimes \Omega_{\mathbb{P}^{1}}^{0,1}$, where $\bar{\partial}^{(0)}$ denotes the induced holomorphic structure on $\operatorname{Hom}\left(V^{(0)} \otimes \sigma^{*} V^{(0)}, \mathbb{T}(0)\right)$.

Lemma 2.6. There exists a constant $C_{0}>0$, independent of $\epsilon$, with the following property:

- If $B^{-1} \cdot \Phi^{*} h^{(1)} \leq h^{(0)} \leq B \cdot \Phi^{*} h^{(1)}$ for some $B>1$, then

$$
\left|\Phi^{*} d^{(1)}-d^{(0)}\right|_{h^{(0)}, g} \leq C_{0} B^{4} \epsilon .
$$

Proof. In the following argument, $C_{i}$ denote positive constants independent of $\epsilon$. Let $\partial_{h(i)}^{(i)}$ denote the $(1,0)$-part of $d^{(i)}$, which is determined by $h^{(i)}$ and $\bar{\partial}^{(i)}$. To show 2.19, we have only to estimate $\left|\partial_{h^{(0)}}^{(0)}-\Phi^{*} \partial_{h^{(1)}}^{(1)}\right|_{h^{(0)}, g}$.

Let $e_{1}, \ldots, e_{r}$ be an orthogonal frame of $V^{(0)}$ with respect to $h^{(0)}$. Because $\Phi^{*} h^{(1)}\left(e_{i}, e_{j}\right)=\Phi^{*} \mathcal{S}^{(1)}\left(e_{i} \otimes \sigma^{*} e_{j}\right)$, we have the following estimate for any $i, j$ :

$$
\left|\bar{\partial}\left(\Phi^{*} h^{(1)}\left(e_{i}, e_{j}\right)\right)\right|_{g}=\left|\bar{\partial}^{(0)}\left(\Phi^{*} \mathcal{S}^{(1)}\right)\left(e_{i} \otimes \sigma^{*} e_{j}\right)\right|_{g} \leq C_{1} \epsilon .
$$

Hence, we obtain

$$
\left|\partial\left(\Phi^{*} h^{(1)}\left(e_{i}, e_{j}\right)\right)\right|_{g} \leq C_{1} \epsilon .
$$

Let $\partial_{h^{(1)}}^{(0)}$ denote the $(1,0)$-operator determined by $\Phi^{*} h^{(1)}$ and $\bar{\partial}^{(0)}$. As $B^{-1} \cdot \Phi^{*} h^{(1)}$ $\leq h^{(0)} \leq B \cdot \Phi^{*} h^{(1)}$, and by 2.20 , we have

$$
\left|\partial_{h^{(1)}}^{(0)}-\partial_{h^{(0)}}^{(0)}\right|_{h^{(0)}, g} \leq C_{2} B \epsilon .
$$

Because $\left|\Phi^{*} \bar{\partial}^{(1)}-\bar{\partial}^{(0)}\right|_{h^{(0)}} \leq C_{3} \epsilon$ and $B^{-1} \cdot \Phi^{*} h^{(1)} \leq h^{(0)} \leq B \cdot \Phi^{*} h^{(1)}$, we have

$$
\left|\Phi^{*} \bar{\partial}^{(1)}-\bar{\partial}^{(0)}\right|_{\Phi^{*} h^{(1)}} \leq C_{4} B^{2} \epsilon \text {. }
$$


Hence, we obtain $\left|\Phi^{*} \partial_{h^{(1)}}^{(1)}-\partial_{h^{(1)}}^{(0)}\right|_{\Phi^{*} h^{(1)}} \leq C_{4} B^{2} \epsilon$, which implies

$$
\left|\Phi^{*} \partial_{h^{(1)}}^{(1)}-\partial_{h^{(1)}}^{(0)}\right|_{h^{(0)}} \leq C_{5} B^{4} \epsilon .
$$

Thus, we obtain 2.19).

Lemma 2.7. There exist positive constants $\epsilon_{0}, C_{10}$ and $C_{11}$ such that, for $\epsilon \leq \epsilon_{0}$,

$$
\begin{aligned}
&\left|\Phi^{*} h^{(1)}-h^{(0)}\right|_{h^{(0)}} \leq C_{10} \epsilon, \\
&\left|\Phi^{*} d^{(1)}-d^{(0)}\right|_{h^{(0)}} \leq C_{11} \epsilon .
\end{aligned}
$$

Proof. According to the result in Subsection 11.3 of [23], if $\epsilon_{0}$ is sufficiently small, 2.21 holds for some $C_{10}$. Then we obtain 2.22 from Lemma 2.6 .

2.3.2. Approximation of pure polarized integrable twistor structures. Let $\left(V^{(i)}, \nabla^{(i)}, \mathcal{S}^{(i)}\right)(i=1,2)$ be integrable polarized pure twistor structures. Let $h^{(i)}$ be the hermitian metrics on $V^{(i)}$ corresponding to $\mathcal{S}^{(i)}$. We fix a hermitian metric $\widetilde{g}$ on $\Omega_{\mathbb{P}^{1}}^{1,0}(2 \cdot\{0, \infty\}) \oplus \Omega_{\mathbb{P}^{1}}^{0,1}$. Let $\Phi: V^{(0)} \rightarrow V^{(1)}$ be a $C^{\infty}$-isomorphism such that for some $\epsilon>0$ :

(B1) $\left|\Phi^{*} \nabla^{(1)}-\nabla^{(0)}\right|_{h^{(0)}, \widetilde{g}} \leq \epsilon$ as a $C^{\infty}$-section of

$$
\operatorname{End}\left(V^{(0)}\right) \otimes\left(\Omega_{\mathbb{P}^{1}}^{1,0}(2 \cdot\{0, \infty\}) \oplus \Omega_{\mathbb{P}^{1}}^{0,1}\right) .
$$

Note that this implies (A1) in Subsection 2.3.1

(B2) Conditions (A2) and (A3) are satisfied.

Lemma 2.8. There exists a constant $C_{20}>0$, independent of $\epsilon$, such that if $B^{-1} \cdot \Phi^{*} h^{(1)} \leq h^{(0)} \leq B \cdot \Phi^{*} h^{(1)}$ for some $B>1$, then

$$
\left|\Phi^{*} \mathcal{U}^{(1)}-\mathcal{U}^{(0)}\right|_{h^{(0)}} \leq C_{20} B^{4} \epsilon, \quad\left|\Phi^{*} \mathcal{Q}^{(1)}-\mathcal{Q}^{(0)}\right|_{h^{(0)}} \leq C_{20} B^{4} \epsilon
$$

Proof. In the following argument, $C_{i}$ denote positive constants independent of $\epsilon$. By Lemma 2.7, we have $\left|\Phi^{*} d^{(1)}-d^{(0)}\right|_{h^{(0)}, \widetilde{g}} \leq C_{21} B^{4} \epsilon$. We obtain

$$
\left|\left(\lambda^{-1}\left(\Phi^{*} \mathcal{U}^{(1)}-\mathcal{U}^{(0)}\right)-\left(\Phi^{*} \mathcal{Q}^{(1)}-\mathcal{Q}^{(0)}\right)-\lambda\left(\Phi^{*} \mathcal{U}^{(1) \dagger}-\mathcal{U}^{(0) \dagger}\right)\right) d \lambda / \lambda\right|_{h^{(0)}, \widetilde{g}} \leq C_{22} B^{4} \epsilon .
$$

Then, it is easy to deduce the claim of the lemma.

Lemma 2.9. There exist positive constants $\epsilon_{0}$ and $C_{30}$ such that, for $\epsilon \leq \epsilon_{0}$,

$$
\begin{gathered}
\left|\Phi^{*} h^{(1)}-h^{(0)}\right|_{h^{(0)}} \leq C_{30} \epsilon, \quad\left|\Phi^{*} \mathcal{U}^{(1)}-\mathcal{U}^{(0)}\right|_{h^{(0)}} \leq C_{30} \epsilon, \\
\left|\Phi^{*} \mathcal{Q}^{(1)}-\mathcal{Q}^{(0)}\right|_{h^{(0)}} \leq C_{30} \epsilon .
\end{gathered}
$$

Proof. This can be shown by the argument in the proof of Lemma 2.7. 


\section{$\S 2.4$. Variation of polarized mixed twistor structure and its enrichment}

\subsubsection{Definitions}

Variation of polarized mixed twistor structure. Let $X$ be a complex manifold. Let $\left(V, \mathbb{D}^{\triangle}\right)$ be a variation of $\mathbb{P}^{1}$-holomorphic vector bundle on $\mathbb{P}^{1} \times X$ equipped with an increasing filtration $W$ indexed by $\mathbb{Z}$ in the category of vector bundles, which is $\mathbb{P}^{1}$-holomorphic and $\mathbb{D}^{\triangle}$-flat. If each $\operatorname{Gr}_{n}^{W}(V)$ is a variation of pure twistor structure of weight $n,\left(V, W, \mathbb{D}^{\triangle}\right)$ is called a variation of mixed twistor structure. Assume we are given the following data on $\left(V, W, \mathbb{D}^{\triangle}\right)$, which are $\mathbb{P}^{1}$-holomorphic and $\mathbb{D}^{\triangle}$-flat:

- A tuple $\boldsymbol{f}$ of nilpotent morphisms $f_{j}: V \rightarrow V \otimes \mathbb{T}(-1)(j=1, \ldots, n)$ which are mutually commutative.

- A $(-1)^{w}$-symmetric pairing $\mathcal{S}: V \otimes \sigma^{*} V \rightarrow \mathbb{T}(-w)$.

- For each $P \in X$, the restriction $(V, W, \boldsymbol{f}, \mathcal{S})_{\mid \mathbb{P}^{1} \times\{P\}}$ is a polarized mixed twistor structure of weight $w$ in $n$ variables. (See Subsection 3.48 of [22].)

Then the tuple $\left(V, \mathbb{D}^{\triangle}, W, \boldsymbol{f}, \mathcal{S}\right)$ is called a variation of polarized mixed twistor structure. Since $W$ is determined by $\boldsymbol{f}$ as the weight filtration of $f(\underline{n}):=\sum_{j=1}^{n} f_{j}$ up to shift of indices, we sometimes omit $W$ in notation.

Enrichment. If $\mathbb{D}^{\triangle}$ and the $\mathbb{P}^{1}$-holomorphic structure are extended to a $T \widetilde{T} E$ structure $\widetilde{\mathbb{D}}^{\triangle}$ for which $\boldsymbol{f}$ and $\mathcal{S}$ are flat, $(V, \widetilde{\mathbb{D}} \triangle, W, \boldsymbol{f}, \mathcal{S})$ is called a variation of polarized mixed integrable twistor structure of weight $w$ in $n$ variables. Note that $W$ is automatically $\widetilde{\mathbb{D}}^{\triangle}$-flat.

If moreover $\left(V, \widetilde{\mathbb{D}}^{\triangle}, \mathcal{S}\right)$ is equipped with a real structure $\kappa$ such that $\kappa \circ \gamma^{*} f_{j}=$ $f_{j} \circ \kappa$, then tuple $\left(V, \widetilde{\mathbb{D}}^{\triangle}, W, \boldsymbol{f}, \mathcal{S}, \kappa,-w\right)$ is called a variation of polarized mixed twistor-TERP structure in $n$ variables.

Remark 8. The notion of polarized mixed twistor-TERP structure is different from "mixed TERP structure" defined by Hertling and Sevenheck (Section 9 of [12]).

Split type. Let $\left(V, W, \mathbb{D}^{\triangle}\right)$ be a variation of mixed twistor structure. It is called of split type if it is equipped with a grading $V=\bigoplus V_{m}$ such that (i) it is $\mathbb{P}^{1}$ holomorphic and $\mathbb{D}^{\triangle}$-flat, (ii) $W_{m}=\bigoplus_{p \leq m} V_{p}$. In that case, each $\left(V_{m}, \mathbb{D}^{\triangle}\right)$ is a variation of pure twistor structure of weight $m$. Note that such a grading is uniquely determined because $H^{0}\left(\mathbb{P}^{1}, \mathcal{O}_{\mathbb{P}^{1}}(m)\right)=0$ for any $m<0$.

A variation of polarized mixed twistor structure $\left(V, W, \mathbb{D}^{\triangle}, \boldsymbol{f}, \mathcal{S}\right)$ is called of split type if the underlying variation of mixed twistor structure $\left(V, W, \mathbb{D}^{\triangle}\right)$ is of 
split type with a grading $V=\bigoplus V_{m}$. We can show that:

- $f_{j}\left(V_{p}\right) \subset V_{p-2} \otimes \mathbb{T}(-1)$.

- The restriction of $\mathcal{S}$ to $V_{p} \otimes \sigma^{*} V_{q}$ is 0 unless $p+q=2 w$.

Similarly, a variation of polarized mixed integrable twistor structure is called of split type if the underlying variation of polarized mixed twistor structure is of split type. The grading is flat.

A polarized mixed twistor-TERP structure $(V, W, \nabla, \boldsymbol{f}, \mathcal{S}, \kappa,-w)$ is called of split type if the underlying variation of mixed integrable twistor structure is of split type. For the grading $V=\bigoplus V_{m}$, we have $\kappa\left(\gamma^{*} V_{m}\right)=V_{m}$.

2.4.2. Reduction. Let $\left(V, W, \mathbb{D}^{\triangle}, \boldsymbol{f}, \mathcal{S}\right)$ be a variation of polarized mixed twistor structure of weight $w$ in $n$ variables. We obtain a variation of $\mathbb{P}^{1}$-holomorphic vector bundle $\left(V^{(0)}, \mathbb{D}^{(0) \triangle}\right):=\operatorname{Gr}^{W}\left(V, \mathbb{D}^{\triangle}\right)$, naturally equipped with a grading $V^{(0)}=\bigoplus \mathrm{Gr}_{m}^{W}(V)$ and a filtration $W_{m}^{(0)}=\bigoplus_{p \leq m} \operatorname{Gr}_{p}^{W}(V)$. We have induced morphisms $f_{j}^{(0)}: \mathrm{Gr}_{m}^{W}(V) \rightarrow \mathrm{Gr}_{m-2}^{W}(V) \otimes \mathbb{T}(-1)$, and hence $f_{j}^{(0)}$ : $V^{(0)} \rightarrow V^{(0)} \otimes \mathbb{T}(-1)$. We also obtain induced morphisms $\mathcal{S}^{(0)}: \operatorname{Gr}_{w-m}^{W}(V) \otimes$ $\sigma^{*} \operatorname{Gr}_{w+m}^{W}(V) \rightarrow \mathbb{T}(-w)$, and hence $\mathcal{S}^{(0)}: V^{(0)} \otimes \sigma^{*}\left(V^{(0)}\right) \rightarrow \mathbb{T}(-w)$. It is known that $\left(V^{(0)}, W^{(0)}, \boldsymbol{f}^{(0)}, \mathcal{S}^{(0)}\right)_{\mid \mathbb{P}^{1} \times\{P\}}$ are polarized mixed twistor structures of split type with weight $w$ in $n$ variables. (See 22]. This can be shown directly and easily.) Hence, $\left(V^{(0)}, W^{(0)}, \mathbb{D}^{(0)} \triangle, \boldsymbol{f}^{(0)}, \mathcal{S}^{(0)}\right)$ is a variation of polarized mixed twistor structure of split type with weight $w$ in $n$ variables. It is denoted by $\operatorname{Gr}^{W}\left(V, W, \mathbb{D}^{\triangle}, \boldsymbol{f}, \mathcal{S}\right)$.

If $\left(V, W, \mathbb{D}^{\triangle}, \boldsymbol{f}, \mathcal{S}\right)$ is enriched to an integrable one $\left(V, W, \widetilde{\mathbb{D}}{ }^{\triangle}, \boldsymbol{f}, \mathcal{S}\right)$, the associated $\mathrm{Gr}$ is also integrable. If moreover $\left(V, W, \widetilde{\mathbb{D}}{ }^{\triangle}, \boldsymbol{f}, \mathcal{S}\right)$ is enriched to a variation of polarized mixed twistor-TERP structure, the associated Gr is also enriched to a variation of polarized mixed twistor-TERP structure of split type.

\subsubsection{Splittings}

Preliminaries. Let $\left(V_{i}, W, \mathbb{D}_{i}^{\triangle}\right)(i=1,2)$ be variations of mixed twistor structure on $\mathbb{P}^{1} \times X$ with a morphism $F:\left(V_{1}, W, \mathbb{D}_{1}^{\triangle}\right) \rightarrow\left(V_{2}, W, \mathbb{D}_{2}^{\triangle}\right)$. We $\operatorname{set}\left(V_{i}^{(0)}, \mathbb{D}_{i}^{(0) \triangle}\right)$ $:=\operatorname{Gr}^{W}\left(V_{i}, \mathbb{D}_{i}^{\triangle}\right)$ on which we have the naturally induced filtrations $W^{(0)}$. We also obtain the induced morphism $F^{(0)}:\left(V_{1}^{(0)}, W^{(0)}, \mathbb{D}_{1}^{(0) \triangle}\right) \rightarrow\left(V_{2}^{(0)}, W^{(0)}, \mathbb{D}_{2}^{(0) \triangle}\right)$. The following lemma is standard.

Lemma 2.10. The rank of $F_{\mid(\lambda, P)}$ is independent of $(\lambda, P) \in \mathbb{P}^{1} \times X$. The morphism $F$ is strict with respect to the weight filtration. Hence, Ker $F$ with the induced filtration $W(\operatorname{Ker} F)$ is a mixed twistor structure, and we have the isomorphism $\operatorname{Ker} F^{(0)} \simeq \operatorname{Gr}^{W}(\operatorname{Ker} F)$. 
Proof. If $X$ is a point, the claims are well known and easy to show. Namely, it is shown in Lemma 2.20 of [21] that $(\mathrm{i}) \operatorname{Ker}(F)$ is a subbundle of $V_{1}$, (ii) $F$ is strict with respect to the weight filtrations, i.e., $F\left(W_{l}\left(V_{1}\right)\right)=F\left(V_{1}\right) \cap W_{l}\left(V_{2}\right)$, (iii) $\operatorname{Ker}(F)$ with the induced weight filtration is a mixed twistor structure. We obtain the isomorphism $\operatorname{Ker} F^{(0)} \simeq \operatorname{Gr}^{W}(\operatorname{Ker} F)$ by strictness.

Let us consider the general case. By using flatness, it is easy to show that $\operatorname{rank} F_{\mid(1, P)}$ and $\operatorname{rank} F_{\mid(1, P)}^{(0)}$ are independent of the choice of a point $P \in X$. Hence, the claim of the lemma follows.

Corollary 2.1. Let $\left(V_{i}, W, \mathbb{D}_{i}^{\triangle}\right)(i=0,1, \ldots, m)$ be variations of mixed twistor structure with morphisms $F_{i}:\left(V_{0}, W, \mathbb{D}_{0}^{\triangle}\right) \rightarrow\left(V_{i}, W, \mathbb{D}_{i}^{\triangle}\right)$ for $i=1, \ldots, m$. Then we have the following natural isomorphism of variations of mixed twistor structure:

$$
\operatorname{Gr}^{W}\left(\bigcap_{i=1}^{m} \operatorname{Ker} F_{i}\right) \simeq \bigcap_{i=1}^{m} \operatorname{Ker} F_{i}^{(0)}
$$

Here, $F_{i}^{(0)}$ denote the induced morphisms $V_{0}^{(0)} \rightarrow V_{i}^{(0)}$.

Local splitting. Let $\left(V, W, \mathbb{D}^{\triangle}\right)$ be a variation of mixed twistor structure. Let $\boldsymbol{N}=\left(N_{j} \mid j=1, \ldots, \ell\right)$ be a tuple of morphisms $N_{j}:\left(V, W, \mathbb{D}^{\triangle}\right) \rightarrow\left(V, W, \mathbb{D}^{\triangle}\right) \otimes$ $\mathbb{T}(-1)$ which are mutually commutative. Let $\left(V^{(0)}, W^{(0)}, \mathbb{D}^{(0) \triangle}\right)$ be as above. Let $\boldsymbol{N}^{(0)}=\left(N_{j}^{(0)} \mid j=1, \ldots, \ell\right)$ be the induced commuting tuple of morphisms $N_{j}^{(0)}$ : $\left(V^{(0)}, W^{(0)}, \mathbb{D}^{(0) \triangle}\right) \rightarrow\left(V^{(0)}, W^{(0)}, \mathbb{D}^{(0) \triangle}\right) \otimes \mathbb{T}(-1)$.

We set $\bar{V}:=\operatorname{Hom}\left(V^{(0)}, V\right)$, which is naturally equipped with the operator $\overline{\mathbb{D}}^{\triangle}$ and an induced filtration $\bar{W}$. Let $\bar{N}_{j}:(\bar{V}, \bar{W}, \overline{\mathbb{D}}) \rightarrow(\bar{V}, \bar{W}, \overline{\mathbb{D}}) \otimes \mathbb{T}(-1)(j=$ $1, \ldots, \ell)$ be the morphisms of mixed twistor structures given by $\bar{N}_{j}(f)=N_{j}$ 。 $f-f \circ N_{j}^{(0)}$. Similarly, we set $\bar{V}^{(0)}:=\operatorname{Hom}\left(V^{(0)}, V^{(0)}\right)$, on which we have the naturally induced operator $\overline{\mathbb{D}}^{(0) \triangle}$, filtration $\bar{W}^{(0)}$ and morphisms of mixed twistor structures $\bar{N}_{j}^{(0)}:\left(\bar{V}^{(0)}, \bar{W}^{(0)}, \overline{\mathbb{D}}^{(0)}\right) \rightarrow\left(\bar{V}^{(0)}, \bar{W}^{(0)}, \overline{\mathbb{D}}^{(0)}\right) \otimes \mathbb{T}(-1)$.

We have the natural isomorphism $\operatorname{Gr}^{\bar{W}}(\bar{V}) \simeq \bar{V}^{(0)}$. The induced filtrations and morphisms coincide. According to Corollary 2.1, we have the following isomorphism of variations of mixed twistor structure:

$$
\operatorname{Gr}^{\bar{W}}\left(\bigcap \operatorname{Ker} \bar{N}_{j}\right) \simeq \bigcap \operatorname{Ker} \bar{N}_{j}^{(0)}
$$

Thus, we obtain the following corollary.

Corollary 2.2. Let $(\lambda, P)$ be any point of $\mathbb{C}_{\lambda} \times X$, and let $U$ be a small neighbourhood of $(\lambda, P)$. There exists a $C^{\infty}$-morphism $F: V_{\mid U}^{(0)} \rightarrow V_{\mid U}$ with the following properties: 
- It preserves the weight filtration, and the induced morphism $\mathrm{Gr}^{W}\left(V_{\mid U}^{(0)}\right) \rightarrow$ $\operatorname{Gr}^{W}\left(V_{\mid U}\right)$ is the identity.

- $F \circ N_{j}^{(0)}=N_{j} \circ F$ for $j=1, \ldots, \ell$.

$C^{\infty}$-splitting. Let $\left(V, W, \mathbb{D}^{\triangle}, \boldsymbol{N}\right)$ and $\left(V^{(0)}, W^{(0)}, \mathbb{D}^{(0)} \triangle, \boldsymbol{N}^{(0)}\right)$ be as above.

Lemma 2.11. There exists a $C^{\infty}$-isomorphism $\Phi: V^{(0)} \rightarrow V$ with the following properties:

- $\Phi$ preserves the weight filtration $W$, and $\mathrm{Gr}^{W} \Phi$ is the identity $\mathrm{Gr}^{W}\left(V^{(0)}\right)=$ $\mathrm{Gr}^{W}(V)$.

- $\Phi \circ N_{j}^{(0)}=N_{j} \circ \Phi$ for $j=1, \ldots, \ell$.

Proof. Let $U \subset \mathbb{C}_{\lambda}$ be a compact region with $U \cup \sigma(U)=\mathbb{P}^{1}$. We take a locally finite open covering $U \times X \subset \bigcup_{p \in I} \mathcal{U}_{p}$ such that we have $C^{\infty}$-isomorphisms $\Phi_{\mathcal{U}_{p}}$ : $V_{\mid \mathcal{U}_{p}}^{(0)} \rightarrow V_{\mid \mathcal{U}_{p}}$ as in Corollary 2.2 i.e., $\Phi_{\mathcal{U}_{p}} \circ N_{j}^{(0)}=N_{j} \circ \Phi_{\mathcal{U}_{p}}$ for any $j$. Similarly, we take a locally finite open covering $\sigma(U) \times X^{\dagger} \subset \bigcup_{q \in J} \mathcal{U}_{q}^{\dagger}$ such that we have $C^{\infty}$ isomorphisms $\Phi_{\mathcal{U}_{q}^{\dagger}}: V_{\mid \mathcal{U}_{q}^{\dagger}}^{(0)} \simeq V_{\mid \mathcal{U}_{q}^{\dagger}}$ as in Corollary 2.2. We take a partition of unity $\left\{\chi_{\mathcal{U}_{p}}, \chi_{\mathcal{U}_{q}^{\dagger}} \mid p \in I, q \in J\right\}$ subordinated to the covering $\left\{\mathcal{U}_{p}, \mathcal{U}_{q}^{\dagger} \mid p \in I, q \in J\right\}$ of $\mathbb{P}^{1} \times X$. We obtain the $C^{\infty}$-isomorphism

$$
\Phi:=\sum_{p \in I} \chi_{\mathcal{U}_{p}} \cdot \Phi_{\mathcal{U}_{p}}+\sum_{q \in J} \chi_{\mathcal{U}_{q}^{\dagger}} \cdot \Phi_{\mathcal{U}_{q}^{\dagger}}: V^{(0)} \rightarrow V
$$

By construction, it has the desired property.

\section{$\S 3$. Polarized mixed integrable twistor structure of split type}

\section{§3.1. Basic examples in one variable}

3.1.1. Rank two. Let us recall a basic example studied in Subsection 3.7 .2 of 22 with a minor enhancement. We set $V^{[2]}:=\mathcal{O}(0,-1) \oplus \mathcal{O}(1,0)$. (See Subsection 2.1.3 for $\mathcal{O}(p, q)$.) It is naturally equipped with a meromorphic connection $\nabla^{[2]}$, and $\left(V^{[2]}, \nabla^{[2]}\right)$ is an integrable twistor structure. We put

$$
W_{-2}\left(V^{[2]}\right):=0, \quad W_{-1}\left(V^{[2]}\right)=W_{0}\left(V^{[2]}\right):=\mathcal{O}(0,-1), \quad W_{1}\left(V^{[2]}\right):=V^{[2]}
$$

Let $F^{[2]}: V^{[2]} \rightarrow V^{[2]} \otimes \mathbb{T}(-1)$ be given by

$$
f_{a}^{(1,0)} \mapsto f_{a}^{(0,-1)} \otimes t_{a}^{(-1)}, \quad f_{a}^{(0,-1)} \mapsto 0 \quad(a=0,1, \infty)
$$


A flat morphism $S^{[2]}: V^{[2]} \otimes \sigma^{*} V^{[2]} \rightarrow \mathbb{T}(0)$ is given by the following correspondence:

$$
\begin{array}{cl}
S^{[2]}\left(f_{1}^{(1,0)} \otimes \sigma^{*} f_{1}^{(0,-1)}\right)=\sqrt{-1} t_{1}^{(0)}, & S^{[2]}\left(f_{1}^{(0,-1)} \otimes \sigma^{*} f_{1}^{(1,0)}\right)=-\sqrt{-1} t_{1}^{(0)}, \\
S^{[2]}\left(f_{1}^{(1,0)} \otimes \sigma^{*} f_{1}^{(1,0)}\right)=0, & S^{[2]}\left(f_{1}^{(0,-1)} \otimes \sigma^{*} f_{1}^{(0,-1)}\right)=0 .
\end{array}
$$

Recall that $\left(V^{[2]}, W, F^{[2]}, S^{[2]}\right)$ is a polarized mixed twistor structure of split type in one variable with weight 0 (Lemma 3.90 of 22]). It follows that the tuple $\left(V^{[2]}, W, \nabla^{[2]}, F^{[2]}, S^{[2]}\right)$ is a polarized mixed integrable twistor structure of split type.

3.1.2. Twist. The bundle $V^{[2]}$ is obtained as the gluing of $V_{0}^{[2]}:=V_{\mid \mathbb{C}_{\lambda}}^{[2]}$ and $V_{\infty}^{[2]}:=V_{\mid \mathbb{C}_{\mu}}^{[2]}$. We would like to explain a twist of the gluing given in Subsection 3.7 .2 of 222, related to the construction in Subsection 2.2.3. Let $N:=F^{[2]} \otimes t_{1}^{(1)}$. Let $v \in V_{\mid \lambda}^{[2]}$ for $\lambda \neq 0, \infty$. The induced elements of $V_{0 \mid \lambda}^{[2]}$ and $V_{\infty \mid \mu}^{[2]}$ are denoted by $v$ and $v^{\dagger}$, respectively. The gluing for $V^{[2]}$ is given by $v=v^{\dagger}$. For $y \in \mathbb{C}$, a vector bundle $\widetilde{V}_{y}^{[2]}$ is given by the following twisted gluing:

$$
\exp (\sqrt{-1} y \cdot N) \cdot v=v^{\dagger} .
$$

Since $N$ is flat, we have the naturally induced flat connection $\nabla_{y}^{[2]}$ on $\widetilde{V}_{y}^{[2]}$. We also have the induced pairing $\widetilde{S}_{y}^{[2]}$ on $\left(\widetilde{V}_{y}^{[2]}, \nabla_{y}^{[2]}\right)$ of weight 0 .

For $y \neq 0$, we have a frame of $\widetilde{V}_{y}^{[2]}$ given as follows:

$$
\begin{gathered}
\widetilde{s}_{1}:=\sqrt{-1} \lambda \cdot f_{0}^{(1,0)}+\sqrt{-1} y \cdot f_{0}^{(0,-1)}=f_{\infty}^{(1,0)}, \\
\widetilde{s}_{2}:=f_{0}^{(1,0)}=-\sqrt{-1} \mu \cdot f_{\infty}^{(1,0)}-\sqrt{-1} y \cdot f_{\infty}^{(0,-1)} .
\end{gathered}
$$

In particular, $\left(\widetilde{V}_{y}^{[2]}, \nabla_{y}^{[2]}\right)$ is a pure integrable twistor structure of weight 0 for any $y \neq 0$. If $y$ is a positive real number, $\widetilde{S}_{y}^{[2]}$ gives a polarization of $\left(\widetilde{V}_{y}^{[2]}, \nabla_{y}^{[2]}\right)$ (Lemma 3.91 of [22]). Actually, $\widetilde{s}_{i}(i=1,2)$ give an orthogonal frame:

$$
\widetilde{S}_{y}^{[2]}\left(\widetilde{s}_{i}, \sigma^{*} \widetilde{s}_{i}\right)=y \quad(i=1,2), \quad \widetilde{S}_{y}^{[2]}\left(\widetilde{s}_{1}, \sigma^{*} \widetilde{s}_{2}\right)=0 .
$$

Note that $\nabla_{y}^{[2]}$ is logarithmic with respect to the lattice $\widetilde{V}_{y}^{[2]}$. For any $y \neq 0$, we have the decomposition

$$
\nabla_{y}^{[2]}=d_{y}^{[2]}-\mathcal{Q}_{y}^{[2]} \frac{d \lambda}{\lambda}
$$

Here, $d_{y}^{[2]}$ is a natural flat connection on $V_{y}^{[2]} \simeq \mathcal{O}_{\mathbb{P}^{1}}(0)^{\oplus 2}$. Let us calculate $\mathcal{Q}^{[2]}$. By easy calculations, we can check

$$
\nabla_{y}^{[2]} \widetilde{s}_{1}=0, \quad \nabla_{y}^{[2]} \widetilde{s}_{2}=\widetilde{s}_{2} \cdot\left(-\frac{d \lambda}{\lambda}\right) .
$$


Hence, $\mathcal{Q}^{[2]}$ is expressed by the following matrix with respect to the frame $\widetilde{s}_{1}, \widetilde{s}_{2}$ :

$$
\left(\begin{array}{ll}
0 & 0 \\
0 & 1
\end{array}\right)
$$

In particular, the eigenvalues are independent of $y$.

Remark 9. For our application, we essentially need only the case in which $y$ is a positive real number. Recall that we have considered a twisted isomorphism (2.16). We will use the above considerations by setting $y=-\sum_{i=1}^{\ell} \log \left|z_{i}\right|^{2}$.

3.1.3. Rank $\ell$. For any positive integer $\ell$, we set

$$
\left(V^{[\ell]}, \nabla^{[\ell]}\right):=\operatorname{Sym}^{\ell-1}\left(V^{[2]}, \nabla^{[2]}\right)
$$

which is equipped with a morphism $F^{[\ell]}: V^{[\ell]} \rightarrow V^{[\ell]} \otimes \mathbb{T}(-1)$ and a pairing $S^{[\ell]}: V^{[\ell]} \otimes \sigma^{*} V^{[\ell]} \rightarrow \mathbb{T}(0)$. For any $y \in \mathbb{C}$, we obtain an integrable twistor structure $\left(\widetilde{V}_{y}^{[\ell]}, \nabla_{y}^{[\ell]}, \widetilde{S}_{y}^{[\ell]}\right)$ with a pairing of weight 0 , by the procedure of Subsection 3.1.2. It is also obtained as the $(\ell-1)$-th symmetric product of $\left(\widetilde{V}_{y}^{[2]}, \nabla_{y}^{[2]}, \widetilde{S}_{y}^{[2]}\right)$. Hence, $\left(\widetilde{V}_{y}^{[\ell]}, \nabla_{y}^{[\ell]}\right)$ is pure with weight 0 for each $y \neq 0$, and $\widetilde{S}_{y}^{[\ell]}$ gives a polarization for each $y>0$. We have the decomposition

$$
\nabla_{y}^{[\ell]}=d_{y}^{[\ell]}-\mathcal{Q}^{[\ell]} \frac{d \lambda}{\lambda}
$$

Let $y \neq 0$. A frame of $\widetilde{V}_{y}^{[\ell]}$ is given by symmetric products $\widetilde{s}_{p}^{[\ell]}:=\widetilde{s}_{1}^{\ell-1-p} \cdot \widetilde{s}_{2}^{p}$ $(p=0,1, \ldots, \ell-1)$, for which $\mathcal{Q}^{[\ell]}$ is expressed by the diagonal matrix whose $p$-th entry is $p(p=0,1, \ldots, \ell-1)$. In particular, the eigenvalues are independent of $y$.

\section{§3.2. Twistor nilpotent orbits of split type and their new supersymmetric indices}

3.2.1. One variable case. Let $\left(V, W, \mathbb{D}^{\triangle}, N, S\right)$ be a variation of polarized mixed twistor structure of split type with weight 0 in one variable on $\mathbb{P}^{1} \times Y$, where $Y$ is a complex manifold. The following lemma is essentially the same as Corollary 3.97 of 22 .

Proposition 3.1. There exist variations of polarized pure twistor structure $\left(U_{\ell}, \mathbb{D}_{\ell}^{\triangle}, S_{\ell}\right)$ of weight 0 on $\mathbb{P}^{1} \times Y$ for $\ell \geq 1$ such that $(\mathrm{i})\left(V, \mathbb{D}^{\triangle}\right) \simeq$ $\bigoplus_{\ell \geq 1}\left(U_{\ell}, \mathbb{D}_{\ell}^{\triangle}\right) \otimes V^{[\ell]}$, (ii) $N=\bigoplus \operatorname{id}_{U_{\ell}} \otimes F^{[\ell]}$ and $S=\bigoplus S_{\ell} \otimes S^{[\ell]}$ under the isomorphism. If $\left(V, W, \mathbb{D}^{\triangle}, N, S\right)$ is enriched to be integrable, $\left(U_{\ell}, \mathbb{D}^{\triangle}, S_{\ell}\right)$ are also enriched to be integrable. 
Proof. We have the grading $V=\bigoplus_{j \in \mathbb{Z}} V_{j}$. For each $j \geq 0$, we set $P V_{j}:=$ $\operatorname{Ker}\left(N^{j+1}: V_{j} \rightarrow V_{-j-2} \otimes \mathbb{T}(j+1)\right)$. It is a variation of pure twistor structure of weight $j$, and equipped with the induced polarization $S_{j}$. For $\ell \geq 1$, we set $U_{\ell}:=P V_{\ell-1} \otimes \mathcal{O}(0,-\ell+1)$, which are naturally variations of polarized pure twistor structure. Now, it is easy to observe that $V$ has the desired decomposition. The integrable case is also easy.

Let $q: Y \times \mathbb{C}^{*} \rightarrow Y$ denote the projection. We have the variations of polarized pure twistor structure on $\mathbb{P}^{1} \times\left(Y \times \mathbb{C}^{*}\right)$ obtained as the pull back of $\left(U_{\ell}, \mathbb{D}_{\ell}, S_{\ell}\right)$, denoted by $q^{*}\left(U_{\ell}, \mathbb{D}_{\ell}^{\Delta}, S_{\ell}\right)$. Recall the construction of Subsection 2.2.3. We obtain the following naturally defined isomorphism:

$$
\operatorname{TNIL}\left(V, \mathbb{D}^{\triangle}, N, S\right) \simeq \bigoplus_{\ell} q^{*}\left(U_{\ell}, \mathbb{D}_{\ell}^{\triangle}, S_{\ell}\right) \otimes \operatorname{TNIL}\left(V^{[\ell]}, \nabla^{[\ell]}, F^{[\ell]}, S^{[\ell]}\right) .
$$

By using the result in Subsection 3.1, we deduce the following:

Proposition 3.2. Set

$$
X_{+}:=Y \times\{z \in \mathbb{C}|0<| z \mid<1\}, \quad X_{-}:=Y \times\{z \in \mathbb{C}|| z \mid>1\} .
$$

Then $\operatorname{TNIL}\left(V, \mathbb{D}^{\Delta}, N\right)$ is a variation of pure integrable twistor structure on $\mathbb{P}^{1} \times$ $\left(X_{+} \cup X_{-}\right)$, and the restriction $\operatorname{TNIL}\left(V, \mathbb{D}^{\triangle}, N, S\right)_{\mid \mathbb{P}^{1} \times X_{+}}$is a twistor nilpotent orbit.

Assume that $\left(V, \mathbb{D}^{\Delta}\right)$ is enriched to integrable $\left(V, \widetilde{\mathbb{D}}^{\triangle}\right)$ such that $S$ and $N$ are $\widetilde{\mathbb{D}}^{\triangle}$-flat. Let $\mathcal{Q}$ and $\mathcal{Q}^{[\ell]}$ be the new supersymmetric indices of $\operatorname{TNIL}\left(V, \widetilde{\mathbb{D}}^{\Delta}, N\right)$ and $\operatorname{TNIL}\left(V^{[\ell]}, \nabla^{[\ell]}, F^{[\ell]}\right)$, respectively. We also have the new supersymmetric index $\mathcal{Q}_{\ell}$ of $\left(U_{\ell}, \widetilde{\mathbb{D}}_{\ell}^{\Delta}\right)$. By construction, we have the following equality, under the isomorphism 3.1):

$$
\mathcal{Q}=\bigoplus_{\ell \geq 1}\left(\mathcal{Q}_{\ell} \otimes \mathrm{id}+\mathrm{id} \otimes \mathcal{Q}^{[\ell]}\right)
$$

The eigenvalues of $\mathcal{Q}$ are easily calculable, once we know those of $\mathcal{Q}_{\ell}$. In particular, we obtain the following.

Corollary 3.1. The eigenvalues of $\mathcal{Q}_{\mid q^{-1}(y)}$ are constant for any $y \in Y$, where $q: X_{+} \cup X_{-} \rightarrow Y$ denotes the projection.

3.2.2. Several variables case. Let $\left(V, W, \mathbb{D}_{V}^{\triangle}, \boldsymbol{N}, S\right)$ be a variation of polarized mixed twistor structure of split type with weight 0 in $n$ variables on $\mathbb{P}^{1} \times Y$. We have the associated variation of twistor structure $\operatorname{TNIL}\left(V, \mathbb{D}_{V}^{\Delta}, \boldsymbol{N}, S\right)$ with a 
pairing of weight 0 on $\mathbb{P}^{1} \times\left(\mathbb{C}^{*}\right)^{n} \times Y$. We set

$$
X^{*}:=\left\{\left(z_{1}, \ldots, z_{n}\right) \in \mathbb{C}^{n}|0<| z_{i} \mid<1\right\} \times Y .
$$

Proposition 3.3. $\operatorname{TNIL}\left(V, \mathbb{D}_{V}^{\triangle}, \boldsymbol{N}, S\right)_{\mid \mathbb{P}^{1} \times X^{*}}$ is a twistor nilpotent orbit.

Proof. For any $\boldsymbol{a} \in \mathbb{R}_{>0}^{n}$, we set $N(\boldsymbol{a}):=\sum_{i=1}^{n} a_{i} N_{i}$. We obtain a variation of mixed polarized twistor structure $\left(V, W, \mathbb{D}^{\triangle}, N(\boldsymbol{a}), S\right)$ of split type with weight 0 in one variable on $\mathbb{P}^{1} \times Y$. Applying the result of Subsection 3.1 .3 to $\left(V, W, \mathbb{D}_{V}^{\triangle}, N(\boldsymbol{a}), S\right)$, we obtain the desired property of $\left(V, W, \mathbb{D}_{V}^{\triangle}, \boldsymbol{N}, S\right)$.

Definition 3.1. An (integrable) twistor nilpotent orbit is called of split type if it is associated to (integrable) polarized mixed twistor structures of split type.

If $\left(V, W, \mathbb{D}_{V}^{\triangle}, \boldsymbol{N}, S\right)$ is enriched to integrable $\left(V, W, \widetilde{\mathbb{D}}_{V}^{\triangle}, \boldsymbol{N}, S\right)$, the associated twistor nilpotent orbit is also enriched to integrable $\operatorname{TNIL}\left(V, \widetilde{\mathbb{D}}_{V}^{\triangle}, \boldsymbol{N}, S\right)$. Let us consider its new supersymmetric index $\mathcal{Q}$. For any $\boldsymbol{a} \in \mathbb{R}_{>0}^{n}$, we set $N(\boldsymbol{a}):=$ $\sum_{i=1}^{n} a_{i} N_{i}$. According to Proposition 3.1. there exist variations of polarized pure integrable twistor structure $\left(U_{\boldsymbol{a}, \ell}, \widetilde{\mathbb{D}}_{\boldsymbol{a}, \ell}^{\triangle}\right)$ for $\ell \geq 1$ such that

$$
\left(V, \widetilde{\mathbb{D}}_{V}^{\triangle}, N(\boldsymbol{a})\right) \simeq \bigoplus_{\ell \geq 1}\left(U_{\boldsymbol{a}, \ell}, \widetilde{\mathbb{D}}_{\boldsymbol{a}, \ell}^{\triangle}\right) \otimes\left(V^{[\ell]}, \nabla^{[\ell]}, F^{[\ell]}\right) .
$$

Lemma 3.1. For any $\boldsymbol{a}, \boldsymbol{b} \in \mathbb{R}_{>0}^{n}$, we have an isomorphism

$$
\left(U_{\boldsymbol{a}, \ell}, \widetilde{\mathbb{D}}_{\boldsymbol{a}, \ell}^{\triangle}\right) \simeq\left(U_{\boldsymbol{b}, \ell}, \widetilde{\mathbb{D}}_{\boldsymbol{b}, \ell}^{\triangle}\right) .
$$

Proof. Let $V=\bigoplus V_{j}$ be the splitting. For any $\boldsymbol{a} \in \mathbb{R}_{>0}^{n}$ and $j \geq 0$, we set

$$
\left(P V_{j, \boldsymbol{a}}, \widetilde{\mathbb{D}}^{\triangle}\right):=\operatorname{Ker}\left(N(\boldsymbol{a})^{j+1}:\left(V_{j}, \widetilde{\mathbb{D}}^{\triangle}\right) \rightarrow\left(V_{-j-2}, \widetilde{\mathbb{D}}^{\triangle}\right) \otimes \mathbb{T}(-j-1)\right) .
$$

We have only to show that $\left(P V_{j, \boldsymbol{a}}, \widetilde{\mathbb{D}}^{\triangle}\right)$ and $\left(P V_{j, \boldsymbol{b}}, \widetilde{\mathbb{D}}^{\triangle}\right)$ are isomorphic if $\boldsymbol{b}$ is sufficiently close to $\boldsymbol{a}$.

We set $\left(Y_{j, \boldsymbol{a}}, \widetilde{\mathbb{D}}^{\triangle}\right)=\operatorname{Im}\left(N(\boldsymbol{a}):\left(V_{j+2}, \widetilde{\mathbb{D}}^{\triangle}\right) \otimes \mathbb{T}(1) \rightarrow\left(V_{j}, \widetilde{\mathbb{D}}^{\triangle}\right)\right)$. Then we obtain the flat splittings $\left(V_{j}, \widetilde{\mathbb{D}}^{\triangle}\right)=\left(P V_{j, \boldsymbol{a}}, \widetilde{\mathbb{D}}^{\triangle}\right) \oplus\left(Y_{j, \boldsymbol{a}}, \widetilde{\mathbb{D}}^{\triangle}\right)$. If $\boldsymbol{b}$ is sufficiently close to $\boldsymbol{a}$, flat isomorphisms $P V_{j, \boldsymbol{a}} \rightarrow P V_{j, \boldsymbol{b}}$ are induced by inclusions and projections. Thus, we are done.

By Lemma 3.1 and the result in Subsection 3.2.1 the eigenvalues of $\mathcal{Q}$ are easily calculable once we know the new supersymmetric indices of $\left(U_{\boldsymbol{a}, \ell}, \widetilde{\mathbb{D}}_{\boldsymbol{a}, \ell}^{\triangle}\right)$ for $\boldsymbol{a} \in \mathbb{R}_{>0}^{\ell}$ and $\ell \geq 1$. In particular, we obtain the following.

Corollary 3.2. The eigenvalues of $\mathcal{Q}_{\mid q^{-1}(y)}$ are constant for any $y \in Y$, where $q: X^{*} \rightarrow Y$ denotes the natural projection. 


\section{$\S 4$. Integrable twistor nilpotent orbits}

\section{$\S 4.1$. Statements}

4.1.1. Twistor nilpotent orbits and polarized mixed twistor structures. Let $Y$ be a complex manifold. Let $\left(V, \mathbb{D}_{V}^{\triangle}\right)$ be a variation of twistor structure on $\mathbb{P}^{1} \times Y$ equipped with the following $\mathbb{P}^{1}$-holomorphic $\mathbb{D}_{V}^{\triangle}$-flat data:

- A pairing $S: V \otimes \sigma^{*} V \rightarrow \mathbb{T}(-w)$ of weight $w$.

- A tuple $\boldsymbol{N}$ of nilpotent morphisms $N_{j}: V \rightarrow V \otimes \mathbb{T}(-1)(j=1, \ldots, n)$ which are mutually commutative.

- $S\left(N_{j} \otimes \mathrm{id}\right)+S\left(\mathrm{id} \otimes \sigma^{*} N_{j}\right)=0$ for $j=1, \ldots, n$.

For simplicity of statement, we assume the following:

- $Y$ is contained in another complex manifold $Y^{\prime}$ as a relatively compact subset, and $\left(V, \mathbb{D}_{V}^{\triangle}, S, \boldsymbol{N}\right)$ is extended onto $Y^{\prime}$.

We set $X^{*}(R):=\left\{\left(z_{1}, \ldots, z_{n}\right)|0<| z_{i} \mid<R\right\} \times Y$.

Theorem 4.1. $\left(V, \mathbb{D}_{V}^{\triangle}, \boldsymbol{N}, S\right)$ is a variation of polarized mixed twistor structure with weight $w$ in $n$ variables if and only if

$$
\operatorname{TNIL}\left(V, \mathbb{D}_{V}^{\triangle}, \boldsymbol{N}, S\right)_{\mid \mathbb{P}^{1} \times X^{*}(R)}
$$

is a twistor nilpotent orbit with weight $w$ for some $R>0$.

Note that the "if" part follows from Theorem 12.22 of [22. The "only if" part immediately follows from Proposition 4.1 below and a result in Subsection 11.3 of 23. (We apply Proposition 4.1 to each point of $Y^{\prime}$.)

Remark 10. The one-dimensional case was proved in Proposition 3.105 of 22 . Such an equivalence for Hodge structure was established by Cattani-KaplanSchmid and Kashiwara-Kawai.

Corollary 4.1. Let $\left(V, \mathbb{D}_{V}^{\triangle}, \boldsymbol{N}, S\right)$ be as above.

- Assume that $\left(V, \mathbb{D}_{V}^{\triangle}\right)$ is enriched to integrable $\left(V, \widetilde{\mathbb{D}}_{V}^{\triangle}\right)$ such that $\boldsymbol{N}$ and $S$ are flat with respect to $\widetilde{\mathbb{D}}_{V}^{\Delta}$. Then $\left(V, \widetilde{\mathbb{D}}_{V}^{\Delta}, N, S\right)$ is a variation of polarized mixed integrable twistor structure with weight $w$ in $n$ variables if and only if $\operatorname{TNIL}\left(V, \widetilde{\mathbb{D}}_{V}^{\triangle}, \boldsymbol{N}, S\right)_{\mathbb{P}^{1} \times X^{*}(R)}$ is an integrable twistor nilpotent orbit for some $R>0$.

- Assume moreover that $\left(V, \widetilde{\mathbb{D}}_{V}^{\triangle}, S\right)$ is equipped with a real structure $\kappa$ which is compatible with $\boldsymbol{N}$. Then $\left(V, \widetilde{\mathbb{D}}_{V}^{\triangle}, \boldsymbol{N}, S, \kappa,-w\right)$ is a variation of polarized mixed twistor-TERP structure if and only if $\operatorname{TNIL}\left(V, \widetilde{\mathbb{D}}_{V}^{\Delta}, \boldsymbol{N}, \mathcal{S}, \kappa,-w\right)$ is a twistorTERP nilpotent orbit on $X^{*}(R)$ for some $R>0$. 
Remark 11. As the one variable case of Corollary 4.1, we obtain the correspondence between twistor-TERP nilpotent orbits and polarized mixed twistor-TERP structures. This is different from the correspondence between mixed TERP structures and HS-orbits in the regular singular case established by Hertling and Sevenheck ([10] and [12]).

4.1.2. Construction of an approximating $C^{\infty}$-isomorphism. Let $(V, W$, $\left.\mathbb{D}^{\Delta}, \boldsymbol{N}, S\right)$ be a variation of polarized mixed twistor structure of weight 0 in $n$ variables on $\mathbb{P}^{1} \times Y$. We obtain a variation of polarized mixed twistor structure of split type $\left(V^{(0)}, W^{(0)}, \mathbb{D}^{(0)} \Delta, \boldsymbol{N}^{(0)}, S^{(0)}\right)$ by taking Gr with respect to the weight filtration, as explained in Subsection 2.4.2. We obtain the families of $\mathbb{P}^{1}$ holomorphic vector bundles $\left(\mathcal{V}^{\triangle}, \mathbb{D}^{\Delta}\right):=\operatorname{TNIL}\left(V, \mathbb{D}^{\Delta}, \boldsymbol{N}\right)$ and $\left(\mathcal{V}^{(0) \triangle}, \mathbb{D}^{(0) \triangle}\right):=$ $\operatorname{TNIL}\left(V^{(0)}, \mathbb{D}^{(0) \Delta}, \boldsymbol{N}^{(0)}\right)$ on $\left(\mathbb{C}^{*}\right)^{n} \times Y$. They are equipped with the induced pairings $\mathcal{S}$ and $\mathcal{S}^{(0)}$. By the result in Subsection $3.2 .2 .\left(\mathcal{V}^{(0)}, \mathbb{D}^{(0) \triangle}, \mathcal{S}^{(0)}\right)$ is a variation of polarized pure twistor structure on $\mathbb{P}^{1} \times X^{*}(1)$. Let $h^{(0)}$ be the corresponding pluri-harmonic metric.

We take a $C^{\infty}$-isomorphism $\Phi: V^{(0)} \rightarrow V$ as in Lemma 2.11, i.e., it satisfies (i) $\Phi \circ N_{i}^{(0)}=N_{i} \circ \Phi$ for $i=1, \ldots, n$, (ii) $\Phi$ preserves the weight filtration $W$, and $\mathrm{Gr}^{W} \Phi$ is the identity of $\mathrm{Gr}^{W}\left(V^{(0)}\right)=\mathrm{Gr}^{W}(V)$. By the property (i) of $\Phi$ and the construction of $\mathcal{V}^{\triangle}$ and $\mathcal{V}^{(0) \triangle}$, we obtain a naturally induced $C^{\infty}$-isomorphism $\widetilde{\Phi}: \mathcal{V}^{(0) \triangle} \rightarrow \mathcal{V}^{\triangle}$.

Let $\bar{\partial}_{\mathcal{V} \triangle \mathbb{P}^{1}}$ denote the $\mathbb{P}^{1}$-holomorphic structure of $\mathcal{V}^{\Delta}$. We use the symbol $\bar{\partial}_{\mathcal{V}^{(0) \Delta, \mathbb{P}^{1}}}$ in a similar meaning. We obtain the following $C^{\infty}$-section of $\operatorname{End}\left(\mathcal{V}^{(0) \triangle}\right) \otimes$ $\Omega_{\mathbb{P}^{1}}^{0,1}$ on $\mathbb{P}^{1} \times X^{*}(1)$ :

$$
F:=\bar{\partial}_{\mathcal{V}^{(0) \triangle}, \mathbb{P}^{1}}-\widetilde{\Phi}^{*}\left(\bar{\partial}_{\mathcal{V}^{\triangle}, \mathbb{P}^{1}}\right) .
$$

We also obtain the following $C^{\infty}$-morphism:

$$
G:=\mathcal{S}^{(0)}-\widetilde{\Phi}^{*} \mathcal{S}: \mathcal{V}^{(0) \triangle} \otimes \sigma^{*} \mathcal{V}^{(0) \triangle} \rightarrow \mathbb{T}(0)
$$

We fix a Kähler metric $g$ on $\mathbb{P}^{1}$. Although the following proposition may look only auxiliary, it means that $\left(\mathcal{V}^{(0) \triangle}, \mathbb{D}^{(0) \triangle}, \mathcal{S}^{(0)}\right)$ approximates $\left(\mathcal{V}^{\triangle}, \mathbb{D}^{\triangle}, \mathcal{S}\right)$ via $\widetilde{\Phi}$ around $\mathbb{P}^{1} \times\{0\} \times Y$. We will prove it in Subsection 4.2.1.

Proposition 4.1. For any $P \in Y$, there exist a positive constant $R_{P}>0$ and a neighbourhood $U_{P}$ of $P$ in $Y$ such that the following estimates hold on $\mathbb{P}^{1} \times$ $\left\{\left(z_{1}, \ldots, z_{n}\right)|0<| z_{j} \mid<R_{P}\right\} \times U_{P}$ :

$$
|F|_{h^{(0)}, g}=O\left(\sum_{j=1}^{n}\left(-\log \left|z_{j}\right|\right)^{-1 / 2}\right),
$$




$$
\begin{aligned}
|G|_{h^{(0)}} & =O\left(\sum_{j=1}^{n}\left(-\log \left|z_{j}\right|\right)^{-1 / 2}\right), \\
\left|\bar{\partial}_{\mathcal{V}^{(0) \triangle, \mathbb{P}^{1}}} G\right|_{h^{(0)}, g} & =O\left(\sum_{j=1}^{n}\left(-\log \left|z_{j}\right|\right)^{-1 / 2}\right) .
\end{aligned}
$$

4.1.3. Estimate of the new supersymmetric index. Assume $\left(V, \mathbb{D}_{V}^{\Delta}, \boldsymbol{N}, S\right)$ is enriched to integrable $\left(V, \widetilde{\mathbb{D}}_{V}^{\Delta}, \boldsymbol{N}, S\right)$. By taking Gr with respect to the weight filtration, we obtain a polarized mixed integrable twistor structure of split type $\left(V^{(0)}, \mathbb{D}_{V}^{(0) \triangle}, \boldsymbol{N}^{(0)}, S^{(0)}\right)$. According to Corollary 4.1 , we have the associated nilpotent orbits:

$$
\begin{aligned}
\left(\mathcal{V}, \widetilde{\mathbb{D}}^{\Delta}, \mathcal{S}\right) & =\operatorname{TNIL}\left(V, \widetilde{\mathbb{D}}_{V}^{\Delta}, \boldsymbol{N}, S\right)_{\mid \mathbb{P}^{1} \times X^{*}(R)}, \\
\left(\mathcal{V}^{(0)}, \widetilde{\mathbb{D}}^{(0) \triangle}, \mathcal{S}^{(0)}\right) & =\operatorname{TNIL}\left(V^{(0)}, \widetilde{\mathbb{D}}_{V}^{(0) \Delta}, \boldsymbol{N}^{(0)}, S^{(0)}\right)_{\mid \mathbb{P}^{1} \times X^{*}(R)} .
\end{aligned}
$$

Let $\mathcal{Q}$ and $h$ (resp. $\mathcal{Q}^{(0)}$ and $h^{(0)}$ ) denote the new supersymmetric index and the pluri-harmonic metric of $\left(\mathcal{V}, \widetilde{\mathbb{D}}^{\Delta}, \mathcal{S}\right)\left(\operatorname{resp} .\left(\mathcal{V}^{(0)}, \widetilde{\mathbb{D}}^{(0) \triangle}, \mathcal{S}^{(0)}\right)\right)$. We will prove the following proposition in Subsection 4.2.2.

Proposition 4.2. Let $\widetilde{\Phi}: \mathcal{V}^{(0)} \rightarrow \mathcal{V}$ be a $C^{\infty}$-isomorphism constructed in Subsection 4.1.2. For any $P \in Y$, there exist $R>0$ and a neighbourhood $U_{P}$ of $P$ in $Y$ such that the following estimates hold with respect to $h^{(0)}$ on $\mathbb{P}^{1} \times\left\{\left(z_{1}, \ldots, z_{n}\right) \mid\right.$ $\left.0<\left|z_{j}\right|<R\right\} \times U_{P}$ :

$$
\widetilde{\Phi}^{*} h-h^{(0)}=O\left(\sum_{i=1}^{n}\left(-\log \left|z_{i}\right|\right)^{-1 / 2}\right), \quad \widetilde{\Phi}^{*} \mathcal{Q}-\mathcal{Q}^{(0)}=O\left(\sum_{i=1}^{n}\left(-\log \left|z_{i}\right|\right)^{-1 / 2}\right) .
$$

In particular, the eigenvalues of $\mathcal{Q}_{\mid q^{-1}(y)}$ are constant up to $O\left(\sum\left(-\log \left|z_{i}\right|\right)^{-\delta}\right)$ for some $\delta>0$, where $q: X^{*}(1) \rightarrow Y$ denotes the natural projection.

\section{$\S 4.2$. Proofs}

4.2.1. Proof of Proposition 4.1. Let $C>0$. Fix $P \in Y$. In the following, we will shrink $Y$ instead of taking a neighbourhood $U_{P}$, for simplicity of description. We set

$$
Z(C):=\left\{\left.\left(z_{1}, \ldots, z_{n}\right) \in\left(\mathbb{C}^{*}\right)^{n}|| z_{i}\right|^{C} \leq\left|z_{i+1}\right|<1, i=1, \ldots, n-1\right\} \times Y .
$$

It is easy to observe that we only have to estimate $F, G$ and $\bar{\partial}_{\mathcal{V}^{(0)} \Delta, \mathbb{P}^{1}} G$ on $\mathbb{P}^{1} \times Z(C)$. For $m=1, \ldots, n$, we put $N^{(0)}(\underline{m}):=\sum_{i \leq m} N_{i}^{(0)}$. Let $W(\underline{m})$ denote the weight filtration of $V^{(0)}$ induced by $N^{(0)}(\underline{m})$. Recall that the filtrations $W(\underline{1}), \ldots, W(\underline{n})$ are compatible (Lemma 3.116 of $[22]$ ). 
We take a compact region $\mathcal{U} \subset \mathbb{C}_{\lambda}$ such that the union of the interior parts of $\mathcal{U}$ and $\sigma(\mathcal{U})$ covers $\mathbb{P}^{1}$. Let $\boldsymbol{v}=\left(v_{i}\right)$ be a frame of $V_{\mid \mathcal{U} \times Y}^{(0)}$ compatible with $W(\underline{1}), \ldots, W(\underline{n})$. For $m=1, \ldots, n$, we set

$$
k_{m}\left(v_{i}\right):=\frac{1}{2} \operatorname{deg}^{W(\underline{m})}\left(v_{i}\right) .
$$

We formally put $k_{0}\left(v_{i}\right)=0$.

Lemma 4.1. Let $A$ be the matrix-valued $(0,1)$-form determined by

$$
-F \boldsymbol{v}=\left(\Phi^{-1} \circ \bar{\partial}_{\mathcal{V} \triangle, \mathbb{P}^{1}} \Phi\right) \boldsymbol{v}=\boldsymbol{v} \cdot A .
$$

Then $A_{i j}=0$ unless $k_{m}\left(v_{i}\right) \leq k_{m}\left(v_{j}\right)(m=1, \ldots, n-1)$ and $k_{n}\left(v_{i}\right)<k_{n}\left(v_{j}\right)$.

Proof. By construction, $\Phi$ preserves the filtrations $W(\underline{m})(m=1, \ldots, n)$, and $\mathrm{Gr}^{W(\underline{n})} \Phi$ is holomorphic. Hence, the claim follows immediately.

Let $q_{0}: \mathbb{C}_{\lambda} \times X^{*}(1) \rightarrow \mathbb{C}_{\lambda} \times Y$ be the projection. Recall $\mathcal{V}_{\mid \mathbb{C}_{\lambda} \times X^{*}(1)}^{(0) \triangle}=q_{0}^{*} V_{0}$ Let $\widetilde{v}_{i}$ be the section of $\mathcal{V}_{\mid \mathcal{U} \times X^{*}(1)}^{(0) \triangle}$ induced by $v_{i}$, and put

$$
\begin{aligned}
v_{j}^{\prime} & :=\widetilde{v}_{j} \prod_{m=1}^{n}\left(-\log \left|z_{m}\right|\right)^{-k_{m}\left(v_{j}\right)+k_{m-1}\left(v_{j}\right)} \\
& =\widetilde{v}_{j} \prod_{m=1}^{n-1}\left(\frac{-\log \left|z_{m}\right|}{-\log \left|z_{m+1}\right|}\right)^{-k_{m}\left(v_{j}\right)}\left(-\log \left|z_{n}\right|\right)^{-k_{n}\left(v_{j}\right)}
\end{aligned}
$$

Due to the norm estimate for tame harmonic bundles (Theorem 13.25 of [22]), the $C^{\infty}$-frame $\boldsymbol{v}^{\prime}=\left(v_{j}^{\prime}\right)$ is adapted to the metric $h^{(0)}$ on $Z(C)$, i.e., the hermitian matrix-valued functions $H=\left(h\left(v_{i}^{\prime}, v_{j}^{\prime}\right)\right)$ and $H^{-1}$ are bounded on $Z(C)$. Let $A^{\prime}$ be the matrix-valued function determined by $-F \boldsymbol{v}^{\prime}=\boldsymbol{v}^{\prime} \cdot A^{\prime}$. Then we have

$$
A_{i j}^{\prime}=A_{i j} \prod_{m=1}^{n-1}\left(\frac{-\log \left|z_{m}\right|}{-\log \left|z_{m+1}\right|}\right)^{k_{m}\left(v_{i}\right)-k_{m}\left(v_{j}\right)}\left(-\log \left|z_{n}\right|\right)^{k_{n}\left(v_{i}\right)-k_{n}\left(v_{j}\right)}
$$

Hence, we obtain $A_{i j}^{\prime}=O\left(\left(-\log \left|z_{n}\right|\right)^{-1 / 2}\right)$. This implies the desired estimate for $F$ on $\mathcal{U} \times Z(C)$. Similarly, we obtain the estimate on $\sigma(\mathcal{U}) \times Z(C)$, and thus on $\mathbb{P}^{1} \times Z(C)$.

Let $\boldsymbol{w}$ be a frame of $V_{\mid \sigma(\mathcal{U}) \times Y^{\dagger}}$ compatible with the filtrations $W(\underline{1}), \ldots, W(\underline{n})$. For $m=1, \ldots, n$, we set

$$
k_{m}\left(w_{i}\right):=\frac{1}{2} \operatorname{deg}^{W(\underline{m})}\left(w_{i}\right)
$$

We formally put $k_{0}\left(w_{i}\right)=0$. We set $G_{0}:=S^{(0)}-\Phi^{*} S: V^{(0)} \otimes \sigma^{*} V^{(0)} \rightarrow \mathbb{T}(0)$. 
Lemma 4.2. $G_{0}\left(v_{i}, \sigma^{*} w_{j}\right)=0$ unless

$$
k_{m}\left(v_{i}\right)+k_{m}\left(w_{j}\right) \geq 0 \quad(m=1, \ldots, n-1), \quad k_{n}\left(v_{i}\right)+k_{n}\left(w_{j}\right)>0 .
$$

Proof. By the relation $S\left(N_{i} \otimes \mathrm{id}\right)+S\left(\mathrm{id} \otimes \sigma^{*}\left(N_{i}\right)\right)=0$, we have

$$
S\left(W_{p}(\underline{m}) \otimes \sigma^{*} W_{q}(\underline{m})\right)=0
$$

unless $p+q \geq 0$. We have similar vanishings for $S^{(0)}$. Note that $\Phi$ preserves the filtrations $W(\underline{m})$ for $m=1, \ldots, n$, and $\operatorname{Gr}^{W(\underline{n})} \Phi$ is compatible with $S$ and $S^{(0)}$. Thus, we obtain the claim.

Let $q_{\infty}: \mathbb{C}_{\mu} \times X^{*}(1)^{\dagger} \rightarrow \mathbb{C}_{\mu} \times Y^{\dagger}$ be the projection. Recall $\mathcal{V}_{\mid \mathbb{C}_{\mu} \times X^{*}(1)^{\dagger}}^{(0) \triangle}=$ $q_{\infty}^{*} V_{\infty}$. Let $\widetilde{w}_{j}$ be the section of $\mathcal{V}_{\mid \sigma(\mathcal{U}) \times X^{*}(1)^{\dagger}}^{(0) \triangle}$ induced by $w_{j}$, and put

$$
w_{j}^{\prime}:=\widetilde{w}_{j} \prod_{m=1}^{n-1}\left(\frac{-\log \left|z_{m}\right|}{-\log \left|z_{m+1}\right|}\right)^{-k_{m}\left(w_{j}\right)}\left(-\log \left|z_{n}\right|\right)^{-k_{n}\left(w_{j}\right)} .
$$

Note that

$$
\begin{aligned}
G\left(v_{i}^{\prime}, \sigma^{*} w_{j}^{\prime}\right)= & G_{0}\left(v_{i}, \sigma^{*} w_{j}\right) \\
& \times \prod_{m=1}^{n-1}\left(\frac{-\log \left|z_{m}\right|}{-\log \left|z_{m+1}\right|}\right)^{-k_{m}\left(v_{i}\right)-k_{m}\left(w_{j}\right)}\left(-\log \left|z_{n}\right|\right)^{-k_{n}\left(v_{i}\right)-k_{n}\left(v_{j}\right)} .
\end{aligned}
$$

Hence, we obtain $|G|_{h^{(0)}}=O\left(\left(-\log \left|z_{n}\right|\right)^{-1 / 2}\right)$. Similarly, we obtain the estimate for $\left|\bar{\partial}_{\mathcal{V}^{(0)}, \mathbb{P}^{1}} G\right|$. Thus, the proof of Proposition 4.1. and hence that of Theorem 4.1. is finished.

4.2.2. Proof of Proposition 4.2. We have the decompositions $\widetilde{\mathbb{D}}^{\Delta}=\mathbb{D}_{\mathcal{V}_{0}}+\nabla_{\lambda}$ and $\widetilde{\mathbb{D}}^{(0) \triangle}=\mathbb{D}_{\mathcal{V}_{0}}^{(0) \triangle}+\nabla_{\lambda}^{(0)}$. By an argument used in the proof of Proposition 4.1 . we can obtain the following estimate with respect to $h^{(0)}$ :

$$
\widetilde{\Phi}^{*} \nabla_{\lambda}-\nabla_{\lambda}^{(0)}=O\left(\sum_{i=1}^{n}\left(-\log \left|z_{i}\right|\right)^{-1 / 2}\right) .
$$

Thus, Proposition 4.2 follows from Lemma 2.9 and Proposition 4.1 .

\section{$\S 5$. Families of meromorphic $\lambda$-flat bundles}

We will review some results on families of meromorphic $\lambda$-flat bundles mainly described in Sections 3 and 4 of [23. See also [20] and 24] for earlier work on asymptotic analysis of meromorphic flat bundles. 


\section{$\S 5.1$. Good lattice on a level}

\subsubsection{Preliminaries}

Good set of irregular values on the level $\boldsymbol{m}$. Let $\Delta^{\ell}$ denote the $\ell$-dimensional multi-disc $\left\{\left(z_{1}, \ldots, z_{\ell}\right)|| z_{i} \mid<1, i=1, \ldots, \ell\right\}$. Put $X:=\Delta^{\ell} \times Y$ for some complex manifold $Y$. Let $D_{i}:=\left\{z_{i}=0\right\}$ and $D:=\bigcup_{i=1}^{\ell} D_{i}$ be hypersurfaces in $X$. Let $M(X, D)$ (resp. $H(X))$ denote the space of meromorphic (resp. holomorphic) functions on $X$ whose poles are contained in $D$. For $\boldsymbol{m}=\left(m_{1}, \ldots, m_{\ell}\right) \in \mathbb{Z}^{\ell}$, we put $\boldsymbol{z}^{\boldsymbol{m}}:=\prod_{i=1}^{\ell} z_{i}^{m_{i}}$.

Let $\boldsymbol{m} \in \mathbb{Z}_{\leq 0}^{\ell} \backslash\{\mathbf{0}\}$. A finite set of meromorphic functions

$$
\mathcal{I}=\left\{\mathfrak{a}=\mathfrak{a}_{\boldsymbol{m}} z^{\boldsymbol{m}}\right\} \subset M(X, D)
$$

is called a good set of irregular values on $(X, D)$ on the level $\boldsymbol{m}$ if the following holds:

- $\mathfrak{a}_{m}$ are holomorphic functions on $X$.

- $\mathfrak{a}_{m}-\mathfrak{b}_{m}$ are nowhere vanishing holomorphic functions on $X$ for any two distinct $\mathfrak{a}, \mathfrak{b} \in \mathcal{I}$.

Let $i(0)$ be the integer such that $m_{i(0)}<0$. If moreover the following condition holds, $\mathcal{I}$ is called a good set of irregular values on $(X, D)$ on the level $(\boldsymbol{m}, i(0))$ :

- $\mathfrak{a}_{\boldsymbol{m}}$ are independent of the variable $z_{i(0)}$ for any $\mathfrak{a} \in \mathcal{I}$.

Remark 12. The first condition is not essential. If we do not impose it, the third condition should be replaced with: $\mathfrak{a}_{\boldsymbol{m}}-\mathfrak{b}_{\boldsymbol{m}}$ are independent of $z_{i(0)}$ for any $\mathfrak{a}, \mathfrak{b} \in \mathcal{I}$.

Multi-sectors and orders on good sets of irregular values on the level $\boldsymbol{m}$. Let $Y, X, D_{i}$ and $D$ be as above. Let $\mathcal{K}$ be a region of $\mathbb{C}_{\lambda}$ or a point in $\mathbb{C}_{\lambda}^{*}$. (For Definition 5.1. we may admit $\mathcal{K}=\{0\}$. Since we do not have to consider Stokes structure in this case, we exclude it in the following.) The product $\mathcal{K} \times X$ is denoted by $\mathcal{X}$. We use the symbols such as $\mathcal{Y}$ and $\mathcal{D}$ in similar meanings. We put $W:=\mathcal{D} \cup(\{0\} \times X)$ in the case $0 \in \mathcal{K}$, and $W:=\mathcal{D}$ otherwise. Let $\pi: \widetilde{\mathcal{X}}(W) \rightarrow \mathcal{X}$ denote the real blow up of $\mathcal{X}$ along $W$, which means in this paper the fiber product of the real blow up $\widetilde{\mathcal{X}}\left(\mathcal{D}_{i}\right)(i=1, \ldots, \ell)$ and $\tilde{\mathcal{X}}(\{0\} \times X)$ over $\mathcal{X}$.

In this paper, a sector of a punctured disc $\Delta^{*}$ means a subset of the form $\left\{z|0<| z \mid<R, \theta_{0} \leq \arg (z) \leq \theta_{1}\right\}$ for some $\theta_{0}<\theta_{1}$. We do not consider sectors whose angles are larger than $2 \pi$ in this paper. 
By a multi-sector of $\mathcal{X}-W$, we mean a subset of the form

$$
U \times \prod_{i=1}^{\ell} S_{i} \times V \quad \text { or } \quad S_{\lambda} \times \prod_{i=1}^{\ell} S_{i} \times V
$$

where

- $U$ denotes a compact region in $\mathcal{K}$. (If $\mathcal{K}$ is a point, $U=\mathcal{K}$.)

- $S_{\lambda}$ denotes a sector of $\mathcal{K}-\{0\}$. (If $0 \notin \mathcal{K}$, we do not consider subsets of the second type.)

- $S_{i}$ denote sectors of $\Delta_{z_{i}}^{*}$.

- $V$ denotes a compact region in $Y$.

For a multi-sector $S$, let $\bar{S}$ denote the closure of $S$ in $\widetilde{\mathcal{X}}(W)$.

Notation. Let $\mathcal{M S}(\mathcal{X}-W)$ denote the set of multi-sectors in $\widetilde{\mathcal{X}}(W)$. For any point $P \in \widetilde{\mathcal{X}}(W)$, let $\mathcal{M S}(P, \mathcal{X}-W)$ denote the set of multi-sectors $S$ such that $P$ is contained in the interior part of $\bar{S}$.

Let $\mathcal{I}$ be a good set of irregular values on $(\mathcal{X}, \mathcal{D})$ on the level $\boldsymbol{m}$. We put $F_{\mathfrak{a}, \mathfrak{b}}:=-\operatorname{Re}\left(\lambda^{-1}(\mathfrak{a}-\mathfrak{b})\right)\left|\lambda \boldsymbol{z}^{-\boldsymbol{m}}\right|$ for any distinct $\mathfrak{a}, \mathfrak{b} \in \mathcal{I}$. They determine $C^{\infty}$ functions on $\widetilde{\mathcal{X}}(W)$.

Notation. Let $A$ be any subset of $\widetilde{\mathcal{X}}(W)$. We write $\mathfrak{a}<_{A} \mathfrak{b}$ for $(\mathfrak{a}, \mathfrak{b}) \in \mathcal{I}^{2}$ if $F_{\mathfrak{a}, \mathfrak{b}}(Q)<0$ for any $Q \in A$. We write $\mathfrak{a} \leq_{A} \mathfrak{b}$ for $(\mathfrak{a}, \mathfrak{b}) \in \mathcal{I}^{2}$ if either $\mathfrak{a}<_{A} \mathfrak{b}$ or $\mathfrak{a}=\mathfrak{b}$. The relation $\leq_{A}$ gives a partial order on $\mathcal{I}$.

We use the symbol $\leq_{P}$ in the case $A=\{P\}$. For a multi-sector $S$, we prefer the symbol $\leq_{S}$ to $\leq_{\bar{S}}$. We also use $\leq_{S}^{\lambda}$ and $\leq_{P}^{\lambda}$ when we emphasize the twist by $\lambda^{-1}$.

For any point $P \in \pi^{-1}(W)$, there exists $S_{P} \in \mathcal{M S}(P, \mathcal{X}-W)$ such that the relations $\leq_{P}$ and $\leq_{S_{P}}$ coincide. Let $\mathcal{M S}(P, \mathcal{X}-W, \mathcal{I})$ denote the set of such $S_{P}$. (The definition of $\mathcal{M S}(P, \mathcal{X}-W, \mathcal{I})$ is slightly different from that in [23.)

5.1.2. Good lattice on the level $\boldsymbol{m}$. Let $Y$ be a complex manifold with a simple normal crossing divisor $D_{Y}^{\prime}$. Let $X:=\Delta_{z}^{k} \times Y, D_{z, i}:=\left\{z_{i}=0\right\}$ and $D_{z}:=\bigcup_{i=1}^{k} D_{z, i}$. We also put $D_{Y}:=\Delta_{z}^{k} \times D_{Y}^{\prime}$ and $D:=D_{z} \cup D_{Y}$. Let $\mathcal{K}$ be a point of $\mathbb{C}_{\lambda}^{*}$ or a compact region in $\mathbb{C}_{\lambda}$. We put $\mathcal{X}:=\mathcal{K} \times X$. We use the symbols $\mathcal{Y}, \mathcal{D}_{z}, \mathcal{D}$ in similar meanings. Let $p_{\lambda}$ denote the projection forgetting the $\mathcal{K}$-component. The completion of $\mathcal{X}$ along $\mathcal{D}_{z}$ is denoted by $\widehat{\mathcal{D}}_{z}$. (See [1, [2] and [19] for completion of complex analytic spaces.) We use the symbol $\widehat{\mathcal{D}}$ in a similar meaning. Let $d_{X}$ denote the restriction of the exterior derivative to the 
$X$-direction. (It will also be denoted just by $d$ frequently, if there is no risk of confusion.)

Let $E$ be a locally free $\mathcal{O}_{\mathcal{X}}$-module with a family of meromorphic flat $\lambda$ connections $\mathbb{D}: E \rightarrow E \otimes p_{\lambda}^{*} \Omega_{X}^{1}(* D)$. Let $\boldsymbol{m} \in \mathbb{Z}_{<0}^{k}$ and $i(0) \in \underline{k}:=\{1, \ldots, k\}$. We put $\boldsymbol{m}(1):=\boldsymbol{m}+\boldsymbol{\delta}_{i(0)}$.

Definition 5.1. We say that $(E, \mathbb{D})$ is an unramifiedly good lattice of a family of meromorphic $\lambda$-flat bundles on the level $(\boldsymbol{m}, i(0))$ if there exists a good set of irregular values $\mathcal{I}$ on the level $(\boldsymbol{m}, i(0))$ on $\left(\mathcal{X}, \mathcal{D}_{z}\right)$, and a decomposition

$$
(E, \mathbb{D})_{\mid \widehat{\mathcal{D}}_{z}}=\bigoplus_{\mathfrak{a} \in \mathcal{I}}\left(\widehat{E}_{\mathfrak{a}}, \widehat{\mathbb{D}}_{\mathfrak{a}}\right)
$$

with $\operatorname{ord}\left(\widehat{\mathbb{D}}_{\mathfrak{a}}-d_{X} \mathfrak{a}\right) \geq \boldsymbol{m}(1)$ in the sense $\left(\widehat{\mathbb{D}}_{\mathfrak{a}}-d_{X} \mathfrak{a}\right) \widehat{E}_{\mathfrak{a}} \subset \boldsymbol{z}^{\boldsymbol{m}(1)} \cdot \widehat{E}_{\mathfrak{a}} \otimes p_{\lambda}^{*} \Omega_{X}^{1}(\log D)$.

The decomposition (5.1) is called the irregular decomposition on the level $(\boldsymbol{m}, i(0))$ (or simply $\boldsymbol{m})$. We also often say that $(E, \mathbb{D})$ is a good lattice on the level $(\boldsymbol{m}, i(0))$ for simplicity.

Remark. As remarked in Remark 2.6.4 of [23], the definition of the order is slightly different from that in [23]. The difference is not essential for our purpose.

If $0 \in \mathcal{K}$, we put $\mathcal{X}^{0}:=\{0\} \times X$ and $\mathcal{D}_{z}^{0}:=\{0\} \times D_{z}$. By shrinking $X$, we obtain the decomposition $(E, \mathbb{D})_{\mid \mathcal{X}^{0}}=\bigoplus_{\mathfrak{a} \in \mathcal{I}}\left(E_{\mathfrak{a}, \mathcal{X}^{0}}, \mathbb{D}_{\mathfrak{a}}^{0}\right)$ whose completion along $\mathcal{D}_{z}^{0}$ is equal to the one induced by 5.1 . It uniquely extends to the $\mathbb{D}$-flat decomposition on the completion $\widehat{\mathcal{X}}^{0}$ of $\mathcal{X}$ along $\mathcal{X}^{0}$ :

$$
(E, \mathbb{D})_{\mid \widehat{\mathcal{X}}^{0}}=\bigoplus_{\mathfrak{a} \in \mathcal{I}}\left(\widehat{E}_{\mathfrak{a}, \widehat{\mathcal{X}}^{0}}, \widehat{\mathbb{D}}_{\mathfrak{a}}\right)
$$

We put $W:=\mathcal{X}^{0} \cup \mathcal{D}_{z}$. Let $\widehat{W}$ denote the completion along $W$. We obtain the decomposition

$$
(E, \mathbb{D})_{\mid \widehat{W}}=\bigoplus_{\mathfrak{a} \in \mathcal{I}}\left(\widehat{E}_{\mathfrak{a}, \widehat{W}}, \widehat{\mathbb{D}}_{\mathfrak{a}}\right)
$$

The decomposition 5.2 is also called the irregular decomposition on the level $(\boldsymbol{m}, i(0))$ in the case $0 \in \mathcal{K}$.

In the following, we formally set $W:=\mathcal{D}_{z}$ if $0 \notin \mathcal{K}$. Let $\pi: \widetilde{\mathcal{X}}(W) \rightarrow \mathcal{X}$ denote the real blow up of $\mathcal{X}$ along $W$. Let $O_{z}$ be the origin of $\Delta_{z}^{k}$, and put $\mathfrak{Z}:=\pi^{-1}\left(O_{z} \times \mathcal{Y}\right)$. We consider the case that $Y=\Delta_{\zeta}^{n}$ and $D_{Y}^{\prime}:=\bigcup_{j=1}^{\ell} D_{\zeta, j}$, where $D_{\zeta, j}:=\left\{\zeta_{j}=0\right\}$. The restriction of $\mathbb{D}$ to the $\Delta_{z}^{k}$-direction is denoted by $\mathbb{D}_{z}$.

Stokes structure on the level $\boldsymbol{m}$. For any multi-sector $S$ in $\mathcal{X}-W$, let $\bar{S}$ denote the closure of $S$ in $\widetilde{\mathcal{X}}(W)$, and let $Z$ denote $\bar{S} \cap \pi^{-1}(W)$. Let $\widehat{Z}$ denote the completion of $\widetilde{\mathcal{X}}(W)$ along $Z$. (See [24] or Subsection 3.1.2 of [23].) The decomposition 
5.2) on $\widehat{W}$ induces the decomposition on $\widehat{Z}$ :

$$
(E, \mathbb{D})_{\mid \widehat{Z}}=\bigoplus_{\mathfrak{a} \in \mathcal{I}}\left(\widehat{E}_{\mathfrak{a}}, \widehat{\mathbb{D}}_{\mathfrak{a}}\right)_{\mid \widehat{Z}}
$$

We put $\mathcal{F}_{\mathfrak{a}}^{Z}:=\bigoplus_{\mathfrak{b} \leq \leq_{S} \mathfrak{a}} \widehat{E}_{\mathfrak{b} \mid \widehat{Z}}$, and then we obtain the filtration $\mathcal{F}^{Z}$ of $E_{\mid \widehat{Z}}$ indexed by $\left(\mathcal{I}, \leq_{S}\right)$. We can show the following proposition. (See Subsection 3.6.1 of [23].)

Proposition 5.1. For any point $P \in \mathfrak{Z}$, there exists $S \in \mathcal{M S}(P, \mathcal{X}-W, \mathcal{I})$ such that:

- There exists a unique $\mathbb{D}$-flat filtration $\mathcal{F}^{S}$ of $E_{\mid \bar{S}}$ indexed by $\left(\mathcal{I}, \leq_{S}\right)$ such that $\mathcal{F}_{\mid \widetilde{Z}}^{S}=\mathcal{F}^{Z}$. Moreover, if a $\mathbb{D}_{z}$-flat filtration $\mathcal{F}^{\prime S}$ of $E_{\mid \bar{S}}$ indexed by $\left(\mathcal{I}, \leq_{S}\right)$ satisfies $\mathcal{F}_{\mid \widehat{Z}}^{\prime S}=\mathcal{F}^{Z}$, then $\mathcal{F}^{\prime S}=\mathcal{F}^{S}$.

- There exists a $\mathbb{D}_{z}$-flat splitting of $\mathcal{F}^{S}$ on $\bar{S}$. Note that if we take such a splitting, the restriction to $\widehat{Z}$ is the same as 5.3 .

We call $\mathcal{F}^{S}$ the Stokes filtration of $(E, \mathbb{D})$ on the level $\boldsymbol{m}$.

Notation. For any $P \in \mathfrak{Z}$, let $\mathcal{M S}^{*}(P, \mathcal{X}-W, \mathcal{I})$ be the set of $S \in \mathcal{M S}(P, \mathcal{X}-W, \mathcal{I})$ as in Proposition 5.1. Let $\mathcal{M S}^{*}(\mathcal{X}-W, \mathcal{I})$ denote the union of $\mathcal{M S}^{*}(P, \mathcal{X}-W, \mathcal{I})$ for $P \in \mathfrak{Z}$.

The following lemma is clear.

Lemma 5.1. Let $S, S^{\prime} \in \mathcal{M S}(P, \mathcal{X}-W, \mathcal{I})$. Assume that (i) $S^{\prime} \subset S$, (ii) $S \in$ $\mathcal{M S}^{*}(P, \mathcal{X}-W, \mathcal{I})$. Then $S^{\prime} \in \mathcal{M S}^{*}(P, \mathcal{X}-W, \mathcal{I})$. The filtration $\mathcal{F}^{S^{\prime}}$ is the restriction of $\mathcal{F}^{S}$

Remark. We can obtain the Stokes filtration on bigger sectors as in Subsection 4.1.2 of [23].

Compatibility of Stokes filtrations. Let $S, S^{\prime} \in \mathcal{M S}^{*}(\mathcal{X}-W, \mathcal{I})$ be such that $S^{\prime} \subset S$. The natural map $\left(\mathcal{I}, \leq_{S}\right) \rightarrow\left(\mathcal{I}, \leq_{S^{\prime}}\right)$ is order-preserving. We can easily show the following lemma by using Proposition 5.1. (See Subsection 3.6.2 of [23].)

Lemma 5.2. The filtrations $\mathcal{F}^{S}$ and $\mathcal{F}^{S^{\prime}}$ are compatible with respect to $\left(\mathcal{I}, \leq_{S}\right)$ $\rightarrow\left(\mathcal{I}, \leq_{S^{\prime}}\right)$ in the following sense:

- $\mathcal{F}_{\mathfrak{a}}^{S^{\prime}}\left(E_{\mid \bar{S}^{\prime}}\right)=\mathcal{F}_{<\mathfrak{a}}^{S^{\prime}}\left(E_{\mid \bar{S}^{\prime}}\right)+\mathcal{F}_{\mathfrak{a}}^{S}\left(E_{\mid \bar{S}}\right)_{\mid \bar{S}^{\prime}}$.

- The induced morphisms $\operatorname{Gr}_{\mathfrak{a}}^{\mathcal{F}^{S}}\left(E_{\mid S}\right)_{\mid S^{\prime}} \rightarrow \operatorname{Gr}_{\mathfrak{a}}^{\mathcal{F}^{S^{\prime}}}\left(E_{\mid S^{\prime}}\right)$ are isomorphisms.

In particular, $\mathcal{F}^{S}\left(E_{\mid \bar{S}}\right)_{\mid \bar{S}^{\prime}}=\mathcal{F}^{S^{\prime}}\left(E_{\mid \bar{S}^{\prime}}\right)$ if $\left(\mathcal{I}, \leq_{S}\right) \rightarrow\left(\mathcal{I}, \leq_{S^{\prime}}\right)$ is an isomorphism. 
Splitting with nice properties. We have the induced morphisms $\operatorname{Res}_{j}(\mathbb{D})$ : $E_{\mid \mathcal{D}_{\zeta, j}} \rightarrow \boldsymbol{z}^{\boldsymbol{m}(1)} E_{\mid \mathcal{D}_{\zeta, j}}$ for $j=1, \ldots, \ell$. Since $\mathcal{F}^{S}$ is $\mathbb{D}$-flat, the restriction $\mathcal{F}_{\mid \mathcal{D}_{\zeta, j}}^{S}$ is preserved by $\operatorname{Res}_{j}(\mathbb{D})$. If we fix the coordinate, we have the induced family of flat $\lambda$-connections on $E_{\mid \mathcal{D}_{\zeta, j}}$, which is denoted by ${ }^{j} \mathbb{D}$. It also preserves the filtration $\mathcal{F}_{\mid \mathcal{D}_{\zeta, j}}^{S}$. Let ${ }^{j} F(j=1, \ldots, \ell)$ be filtrations of $E_{\mid \mathcal{D}_{\zeta, j}}$ which are preserved by the endomorphism $\operatorname{Res}_{j}(\mathbb{D})$ and the flat connection ${ }^{j} \mathbb{D}$ on $E_{\mid \mathcal{D}_{\zeta, j}}$. We can show the following (Subsection 3.6.3 of [23]).

Proposition 5.2. Let $P \in \mathfrak{Z}$. There exist $S \in \mathcal{M S}^{*}(P, \mathcal{X}-W, \mathcal{I})$ and a $\mathbb{D}_{z^{-}}$ flat splitting of the filtration $\mathcal{F}^{S}$ whose restriction to $\bar{S} \cap \mathcal{D}_{\zeta, j}$ is compatible with $\operatorname{Res}_{j}(\mathbb{D})$ and the filtrations ${ }^{j} F$ for $j=1, \ldots, \ell$.

Under some more assumption, we can take a $\mathbb{D}$-flat splitting. (See Subsection 3.6 .3 of 23 .)

Proposition 5.3. Assume that $\mathcal{K}$ is a point or a compact region in $\mathbb{C}_{\lambda}^{*}$. Assume that the eigenvalues $\alpha, \beta$ of $\operatorname{Res}_{j}\left(\mathbb{D}^{f}\right)_{\mid D_{j} \times\{\lambda\}}$ satisfy $\alpha-\beta \notin \mathbb{Z}-\{0\}$ for any $j=1, \ldots, \ell$ and for any $\lambda \in \mathcal{K}$. Then we have a $\mathbb{D}$-flat splitting of $\mathcal{F}^{S}$ whose restriction to $\mathcal{D}_{\zeta, j}$ is compatible with ${ }^{j} F$ for each $j=1, \ldots, \ell$.

Some functoriality of Stokes filtrations. We describe functoriality of Stokes filtrations. See Subsection 3.6.4 of [23] for more details.

In general, when we are given vector spaces $U \subset V$, let $U^{\perp}$ denote the subspace of the dual $V^{\vee}$ given by $U^{\perp}=\left\{f \in V^{\vee} \mid f(U)=0\right\}$. This is naturally generalized for vector bundles. Let $(E, \mathbb{D}, \mathcal{I})$ be an unramifiedly good lattice of a family of meromorphic $\lambda$-flat bundles on the level $(\boldsymbol{m}, i(0))$ on $\left(\mathcal{X}, \mathcal{D}_{z}\right)$, and let $S \in \mathcal{M S}^{*}(\mathcal{X}-W, \mathcal{I})$. We have the following for any $\mathfrak{a} \in \mathcal{I}^{\vee}:=\{-\mathfrak{b} \mid \mathfrak{b} \in \mathcal{I}\}$ :

$$
\mathcal{F}_{\mathfrak{a}}^{S}\left(E_{\mid \bar{S}}^{\vee}\right)=\left(\sum_{\substack{\mathfrak{c} \in \mathcal{I} \\ \mathfrak{c} \nsupseteq S-\mathfrak{a}}} \mathcal{F}_{\mathfrak{c}}^{S}\left(E_{\mid \bar{S}}\right)\right)^{\perp} .
$$

Let $\left(E_{p}, \mathbb{D}_{p}, \mathcal{I}_{p}\right)(p=1,2)$ be good lattices of families of meromorphic $\lambda$-flat bundles on the level $(\boldsymbol{m}, i(0))$. We assume that $\mathcal{I}_{1} \otimes \mathcal{I}_{2}:=\left\{\mathfrak{a}_{1}+\mathfrak{a}_{2} \mid \mathfrak{a}_{p} \in \mathcal{I}_{p}\right\}$ is a good set of irregular values on the level $(\boldsymbol{m}, i(0))$. Let $S \in \bigcap_{p=1,2} \mathcal{M S}^{*}\left(\mathcal{X}-W, \mathcal{I}_{p}\right)$. For each $\mathfrak{a} \in \mathcal{I}_{1} \otimes \mathcal{I}_{2}$ we have

$$
\mathcal{F}_{\mathfrak{a}}^{S}\left(\left(E_{1} \otimes E_{2}\right)_{\mid \bar{S}}\right)=\sum_{\mathfrak{a}_{1}+\mathfrak{a}_{2} \leq S \mathfrak{a}} \mathcal{F}_{\mathfrak{a}_{1}}^{S}\left(E_{1 \mid \bar{S}}\right) \otimes \mathcal{F}_{\mathfrak{a}_{2}}^{S}\left(E_{2 \mid \bar{S}}\right)
$$

Assume that $\mathcal{I}_{1} \oplus \mathcal{I}_{2}:=\mathcal{I}_{1} \cup \mathcal{I}_{2}$ is a good set of irregular values on the level $(\boldsymbol{m}, i(0))$. Let $S \in \bigcap_{p=1,2} \mathcal{M S}^{*}\left(\mathcal{X}-W, \mathcal{I}_{p}\right)$. For each $\mathfrak{a} \in \mathcal{I}_{1} \oplus \mathcal{I}_{2}$ we have

$$
\mathcal{F}_{\mathfrak{a}}^{S}\left(\left(E_{1} \oplus E_{2}\right)_{\mid \bar{S}}\right)=\mathcal{F}_{\mathfrak{a}}^{S}\left(E_{1 \mid \bar{S}}\right) \oplus \mathcal{F}_{\mathfrak{a}}^{S}\left(E_{2 \mid \bar{S}}\right) .
$$


Let $F:\left(E_{1}, \mathbb{D}_{1}\right) \rightarrow\left(E_{2}, \mathbb{D}_{2}\right)$ be a flat morphism. For simplicity, we assume that $\mathcal{I}_{1} \cup \mathcal{I}_{2}$ is a good set of irregular values on the level $(\boldsymbol{m}, i(0))$.

Lemma 5.3. Let $S \in \bigcap_{p=1,2} \mathcal{M S}^{*}\left(\mathcal{X}-W, \mathcal{I}_{p}\right)$. The restriction $F_{\mid \bar{S}}$ preserves the Stokes filtrations. As a result, we obtain the following.

- If the restriction of $F$ to $\mathcal{X}-\mathcal{D}$ is an isomorphism, then $\mathcal{I}_{1}=\mathcal{I}_{2}$ and $\mathcal{F}_{\mathfrak{a}}^{S}\left(E_{1 \mid S \backslash \mathcal{D}}\right)$ $=\mathcal{F}_{\mathfrak{a}}^{S}\left(E_{2 \mid S \backslash \mathcal{D}}\right)$.

- In particular, the Stokes filtration $\mathcal{F}^{S}$ depends only on the family of meromorphic $\lambda$-flat bundles $(E(* \mathcal{D}), \mathbb{D})$ in the sense that it is independent of the choice of an unramifiedly good lattice $E \subset E(* \mathcal{D})$ on the level $(\boldsymbol{m}, i(0))$.

The associated graded bundle on the level $\boldsymbol{m}$. For each $S \in \mathcal{M S}^{*}(\mathcal{X}-W, \mathcal{I})$ and each $\mathfrak{a} \in \mathcal{I}$, we obtain the bundle $\operatorname{Gr}_{\mathfrak{a}}^{\boldsymbol{m}}\left(E_{\mid \bar{S}}\right)$ on $\bar{S}$ associated to the Stokes filtration $\mathcal{F}^{S}$ on the level $\boldsymbol{m}$. By varying $S$ and gluing $\operatorname{Gr}_{\mathfrak{a}}^{\boldsymbol{m}}\left(E_{\mid \bar{S}}\right)$, we obtain the bundle $\operatorname{Gr}_{\mathfrak{a}}^{\boldsymbol{m}}\left(E_{\mid \widetilde{\mathcal{V}}(W)}\right)$ on $\widetilde{\mathcal{V}}(W)$ with the induced family of flat $\lambda$-connections $\mathbb{D}_{\mathfrak{a}}$, where $\mathcal{V}$ denotes some neighbourhood of $O_{z} \times \mathcal{Y}$, and $\widetilde{\mathcal{V}}(W)$ denotes the real blow up of $\mathcal{V}$ along $W \cap \mathcal{V}$. It is known that we have the descent of $\operatorname{Gr}_{\mathfrak{a}}^{m}\left(E_{\mid \widetilde{\mathcal{V}}(W)}\right)$ to $\mathcal{V}$, i.e., there exists a locally free sheaf $\operatorname{Gr}_{\mathfrak{a}}^{\boldsymbol{m}}(E)$ on $\mathcal{V}$ with a family of meromorphic flat $\lambda$-connections $\mathbb{D}_{\mathfrak{a}}$ such that

$$
\pi^{-1}\left(\operatorname{Gr}_{\mathfrak{a}}^{m}(E), \mathbb{D}_{\mathfrak{a}}\right) \simeq\left(\operatorname{Gr}_{\mathfrak{a}}^{m}\left(E_{\mid \widetilde{\mathcal{V}}(W)}\right), \mathbb{D}_{\mathfrak{a}}\right), \quad\left(\operatorname{Gr}_{\mathfrak{a}}^{m}(E), \mathbb{D}_{\mathfrak{a}}\right)_{\mid \widehat{W} \cap \mathcal{V}} \simeq\left(\widehat{E}_{\mathfrak{a}}, \widehat{\mathbb{D}}_{\mathfrak{a}}\right)_{\mid \widehat{W} \cap \mathcal{V}}
$$

(See Subsection 3.6.5 of [23].) If we set $\mathbb{D}_{\mathfrak{a}}^{\prime}:=\mathbb{D}_{\mathfrak{a}}-d_{X} \mathfrak{a}$, we have $\mathbb{D}_{\mathfrak{a}}^{\prime} E_{\mathfrak{a}} \subset \boldsymbol{z}^{\boldsymbol{m}(1)}$. $E_{\mathfrak{a}} \otimes p_{\lambda}^{*} \Omega_{X}^{1}(\log D)$.

Let us give some statements on functoriality. See Subsections 3.6.5 of [23] for more details.

By taking Gr of the Stokes filtrations of $\left(E^{\vee}, \mathbb{D}^{\vee}, \mathcal{I}^{\vee}\right)$, we obtain the associated graded bundle $\operatorname{Gr}^{\boldsymbol{m}}\left(E^{\vee}\right)=\bigoplus_{\mathfrak{a} \in \mathcal{I}^{\vee}} \operatorname{Gr}_{\mathfrak{a}}^{\boldsymbol{m}}\left(E^{\vee}\right)$. We have a natural flat isomorphism

$$
\operatorname{Gr}_{\mathfrak{a}}^{\boldsymbol{m}}\left(E^{\vee}\right) \simeq \operatorname{Gr}_{-\mathfrak{a}}^{\boldsymbol{m}}(E)^{\vee}
$$

Actually, by construction, we have such an isomorphism on the real blow up, which induces (5.4).

Let $\left(E_{p}, \mathbb{D}_{p}, \mathcal{I}_{p}\right)(p=1,2)$ be unramifiedly good lattices of families of meromorphic $\lambda$-flat bundles on the level $(\boldsymbol{m}, i(0))$. Assume $\mathcal{I}_{1} \otimes \mathcal{I}_{2}$ is a good set of irregular values on the level $(\boldsymbol{m}, i(0))$. We have the following natural isomorphism for each $\mathfrak{a} \in \mathcal{I}_{1} \otimes \mathcal{I}_{2}$ :

$$
\operatorname{Gr}_{\mathfrak{a}}^{\boldsymbol{m}}\left(E_{1} \otimes E_{2}\right) \simeq \bigoplus_{\substack{\left(\mathfrak{a}_{1}, \mathfrak{a}_{2}\right) \in \mathcal{I}_{1} \times \mathcal{I}_{2} \\ \mathfrak{a}_{1}+\mathfrak{a}_{2}=\mathfrak{a}}} \operatorname{Gr}_{\mathfrak{a}_{1}}^{\boldsymbol{m}}\left(E_{1}\right) \otimes \operatorname{Gr}_{\mathfrak{a}_{2}}^{\boldsymbol{m}}\left(E_{2}\right)
$$


Assume $\mathcal{I}_{1} \oplus \mathcal{I}_{2}$ is a good set of irregular values on the level $(\boldsymbol{m}, i(0))$. For each $\mathfrak{a} \in \mathcal{I}_{1} \oplus \mathcal{I}_{2}$, we obviously have

$$
\operatorname{Gr}_{\mathfrak{a}}^{\boldsymbol{m}}\left(E_{1} \oplus E_{2}\right) \simeq \operatorname{Gr}_{\mathfrak{a}}^{\boldsymbol{m}}\left(E_{1}\right) \oplus \operatorname{Gr}_{\mathfrak{a}}^{\boldsymbol{m}}\left(E_{2}\right)
$$

Lemma 5.4. Let $F:\left(E_{1}, \mathbb{D}_{1}\right) \rightarrow\left(E_{2}, \mathbb{D}_{2}\right)$ be a flat morphism. Assume $\mathcal{I}_{1} \oplus \mathcal{I}_{2}$ is a good set of irregular values on the level $(\boldsymbol{m}, i(0))$. We have the naturally induced morphism $\operatorname{Gr}_{\mathfrak{a}}^{\boldsymbol{m}}(F): \operatorname{Gr}_{\mathfrak{a}}^{\boldsymbol{m}}\left(E_{1}\right) \rightarrow \operatorname{Gr}_{\mathfrak{a}}^{\boldsymbol{m}}\left(E_{2}\right)$. If the restriction $E_{1 \mid \mathcal{X}-\mathcal{D}} \rightarrow E_{2 \mid \mathcal{X}-\mathcal{D}}$ is an isomorphism, so is the induced morphism

$$
\operatorname{Gr}_{\mathfrak{a}}^{\boldsymbol{m}}\left(E_{1}\right) \otimes \mathcal{O}(* \mathcal{D}) \rightarrow \operatorname{Gr}_{\mathfrak{a}}^{\boldsymbol{m}}\left(E_{2}\right) \otimes \mathcal{O}(* \mathcal{D}) .
$$

Hence, the associated meromorphic flat bundles $\left(\operatorname{Gr}_{\mathfrak{a}}^{m}(E) \otimes \mathcal{O}(* \mathcal{D}), \mathbb{D}_{\mathfrak{a}}\right)$ are well defined for the meromorphic flat bundle $(E(* \mathcal{D}), \mathbb{D})$.

A characterization of sections of $E$. Let $\boldsymbol{w}_{\mathfrak{a}}$ be a frame of $\operatorname{Gr}_{\mathfrak{a}}^{\boldsymbol{m}}(E)$. Let $S \in \mathcal{M S}^{*}(\mathcal{X}-W, \mathcal{I})$, and let $E_{\mid \bar{S}}=\bigoplus E_{\mathfrak{a}, S}$ be a $\mathbb{D}_{z}$-flat splitting of the Stokes filtration $\mathcal{F}^{S}$. By the natural isomorphism $E_{\mathfrak{a}, S} \simeq \operatorname{Gr}_{\mathfrak{a}}^{\boldsymbol{m}}(E)_{\mid \bar{S}}$, we take a lift $\boldsymbol{w}_{\mathfrak{a}, S}$ of $\boldsymbol{w}_{\mathfrak{a}}$. Thus, we obtain a frame $\boldsymbol{w}_{S}=\left(\boldsymbol{w}_{\mathfrak{a}, S}\right)$ of $E_{\mid \bar{S}}$. The following proposition is clear; it implies a characterization of sections of $E$ by growth order with respect to the frames $\boldsymbol{w}_{S}\left(S \in \mathcal{M S}^{*}(\mathcal{X}-W, \mathcal{I})\right)$.

Proposition 5.4. Let $\boldsymbol{v}$ be a frame of $E$, and let $G_{S}$ be determined by $\boldsymbol{v}_{\mid S}=$ $\boldsymbol{w}_{S} \cdot G_{S}$. Then $G_{S}$ and $G_{S}^{-1}$ are bounded on $S$.

Complement on the induced flat connection along the $\lambda$-direction. Assume that we are given a connection along the $\lambda$-direction $\nabla_{\lambda}: E \rightarrow E \otimes \Omega_{\mathcal{K}}^{1}(* W)$ such that $\mathbb{D}^{f}+\nabla_{\lambda}$ is a meromorphic flat connection on $E$.

Lemma 5.5. The Stokes filtrations are flat with respect to $\nabla_{\lambda}$, and we have the induced meromorphic flat connection $\nabla_{\lambda}$ along the $\lambda$-direction on $\operatorname{Gr}_{\mathfrak{a}}^{\boldsymbol{m}}(E)$.

Proof. Take $N$ such that $\lambda^{N} \nabla_{\lambda}\left(\partial_{\lambda}\right) E \subset E \otimes \mathcal{O}_{\mathcal{X}}(* \mathcal{D})$. Let $\boldsymbol{w}_{S}=\left(\boldsymbol{w}_{\mathfrak{a}, S}\right)$ be a frame of $E_{\mid \bar{S}}$ as above. Let $A$ be the matrix-valued holomorphic function on $S$ determined by $\lambda^{N} \nabla\left(\partial_{\lambda}\right) \boldsymbol{w}_{S}=\boldsymbol{w}_{S} \cdot A$. We have the decomposition into blocks $A=\left(A_{\mathfrak{a}, \mathfrak{b}}\right)$ determined by $\lambda^{N} \nabla\left(\partial_{\lambda}\right) \boldsymbol{w}_{\mathfrak{b}, S}=\boldsymbol{w}_{\mathfrak{a}, S} \cdot A_{\mathfrak{a}, \mathfrak{b}}$. By using Proposition 5.4 we can show that $A_{\mathfrak{a}, \mathfrak{b}}$ are of polynomial order.

Let $B_{\mathfrak{a}}$ be the matrix-valued meromorphic one-forms determined by

$$
\mathbb{D}_{\mathfrak{a}, z} \boldsymbol{w}_{\mathfrak{a}}=\boldsymbol{w}_{\mathfrak{a}} \cdot\left(d_{z} \mathfrak{a}+B_{\mathfrak{a}}\right) .
$$

Note that $\boldsymbol{z}^{-\boldsymbol{m}(1)} B_{\mathfrak{a}}$ is logarithmic. By the commutativity $\left[\mathbb{D}^{f}, \nabla_{\lambda}\right]=0$, we obtain the following relation for $\mathfrak{a} \neq \mathfrak{b}$ :

$$
\lambda \cdot d_{z} A_{\mathfrak{a}, \mathfrak{b}}+\left(d_{z}(\mathfrak{a}-\mathfrak{b})\right) \cdot A_{\mathfrak{a}, \mathfrak{b}}+\left(A_{\mathfrak{a}, \mathfrak{b}} B_{\mathfrak{b}}-B_{\mathfrak{a}} A_{\mathfrak{a}, \mathfrak{b}}\right)=0 .
$$


By applying the results in Subsection 20.3 of 23 to (5.6), we obtain $A_{\mathfrak{a}, \mathfrak{b}}=0$ unless $\mathfrak{a} \leq_{S} \mathfrak{b}$, which implies the first claim. Since $A_{\mathfrak{a}, \mathfrak{a}}$ is of polynomial order, the induced connection along the $\lambda$-direction is meromorphic.

Prolongment of morphisms. Let $\left(E_{p}, \mathbb{D}_{p}, \mathcal{I}_{p}\right)(p=1,2)$ be good lattices on the level $(\boldsymbol{m}, i(0))$. Assume that $\mathcal{I}_{1} \cup \mathcal{I}_{2}$ is a good set of irregular values on the level $(\boldsymbol{m}, i(0))$. Assume that we are given a flat morphism $F:\left(E_{1}, \mathbb{D}_{1}\right)_{\mid \mathcal{X}-\mathcal{D}_{z}} \rightarrow$ $\left(E_{2}, \mathbb{D}_{2}\right)_{\mid \mathcal{X}-\mathcal{D}_{z}}$ with the following properties:

- For each small sector $S \in \mathcal{M S}\left(\mathcal{X}-\mathcal{D}_{z}, \mathcal{I}_{1} \cup \mathcal{I}_{2}\right)$, the Stokes filtrations are preserved by $F_{\mid S}$.

- The induced maps $\operatorname{Gr}_{\mathfrak{a}}^{m}(F): \mathrm{Gr}_{\mathfrak{a}}^{m}\left(E_{1}\right)_{\mid \mathcal{X}-\mathcal{D}_{z}} \rightarrow \mathrm{Gr}_{\mathfrak{a}}^{m}\left(E_{2}\right)_{\mid \mathcal{X}-\mathcal{D}_{z}}$ extend to $\operatorname{Gr}_{\mathfrak{a}}^{m}\left(E_{1}\right) \rightarrow \operatorname{Gr}_{\mathfrak{a}}^{m}\left(E_{2}\right)$ for any $\mathfrak{a} \in \mathcal{I}_{1} \cup \mathcal{I}_{2}$.

Lemma 5.6. $F$ extends to a morphism $E_{1} \rightarrow E_{2}$.

Proof. Let $\boldsymbol{w}_{p, S}=\left(\boldsymbol{w}_{p, \mathfrak{a}, S}\right)$ be frames of $E_{p \mid \bar{S}}$ as above. Let $A=\left(A_{\mathfrak{a}, \mathfrak{b}}\right)$ be determined by $F\left(\boldsymbol{w}_{1, S}\right)=\boldsymbol{w}_{2, S} A$. By assumption, $A_{\mathfrak{a}, \mathfrak{b}}=0$ unless $\mathfrak{a} \leq_{S} \mathfrak{b}$, and $A_{\mathfrak{a}, \mathfrak{a}}$ is bounded. By applying an argument in the proof of Lemma 5.5 to $A_{\mathfrak{a}, \mathfrak{b}}$ for $\mathfrak{a}<_{S} \mathfrak{b}$, and by shrinking $X$, we obtain $A_{\mathfrak{a}, \mathfrak{b}}=O\left(\exp \left(-\epsilon\left|\lambda^{-1} z^{m}\right|\right)\right)$ on $S \cap\left(\mathcal{X}-\mathcal{D}_{z}\right)$ for some $\epsilon>0$. Then the claim follows from Proposition 5.4 .

5.1.3. Pseudo-good lattice on the level $\boldsymbol{m}$. Let $Y$ be a complex manifold. Let $X:=\Delta_{z}^{k} \times Y, D_{z, i}:=\left\{z_{i}=0\right\}$ and $D:=\bigcup_{i=1}^{k} D_{z, i}$. (We consider only the case $D_{Y}=\emptyset$, for simplicity.) Let $E$ be a locally free $\mathcal{O}_{X}$-module. For simplicity, we consider a meromorphic flat connection $\nabla: E \rightarrow E \otimes \Omega_{X}^{1}(* D)$ instead of a family of meromorphic flat $\lambda$-connections. Let $\boldsymbol{m} \in \mathbb{Z}_{<0}^{k}$ and $i(0) \in \underline{k}$. We put $\boldsymbol{m}(1):=\boldsymbol{m}+\boldsymbol{\delta}_{i(0)}$.

Definition 5.2. We say that $(E, \nabla)$ is an unramifiedly pseudo-good lattice on the level $(\boldsymbol{m}, i(0))$ if there exists an unramifiedly good lattice $E^{\prime} \supset E$ of $(E(* D), \nabla)$ with the irregular decomposition $\left(E^{\prime}, \nabla\right)_{\mid \widehat{D}}=\bigoplus_{\mathfrak{a} \in \mathcal{I}}\left(\widehat{E}_{\mathfrak{a}}^{\prime}, \widehat{\nabla}_{\mathfrak{a}}\right)$ on the level $(\boldsymbol{m}, i(0))$ such that

$$
E_{\mid \widehat{D}}=\bigoplus_{\mathfrak{a} \in \mathcal{I}}\left(\widehat{E}_{\mathfrak{a}}^{\prime} \cap E_{\mid \widehat{D}}\right) .
$$

The decomposition (5.7) is called the irregular decomposition of $(E, \mathbb{D})$ on the level $(\boldsymbol{m}, i(0))$.

It is easy to observe that $\widehat{E}_{\mathfrak{a}}:=\widehat{E}_{\mathfrak{a}}^{\prime} \cap E_{\mid \widehat{D}}$ in 5.7 is independent of the choice of a good lattice $E^{\prime} \supset E$ on the level $\boldsymbol{m}$. We have straightforward generalizations of the results in Subsection 5.1.2. We naturally identify $X$ with $\{1\} \times X \subset \mathbb{C}_{\lambda} \times X$ when we consider the order $\leq_{S}$ for a multi-sector $S \subset X-D$. 
Construction of Gr. We take an unramifiedly good lattice $E^{\prime} \supset E$ on the level $(\boldsymbol{m}, i(0))$. By shrinking $X$ around $O_{z} \times Y$, we have the vector bundle $\operatorname{Gr}_{\mathfrak{a}}^{\boldsymbol{m}}\left(E^{\prime}\right)$ on $X$ with a meromorphic flat connection $\nabla_{\mathfrak{a}}$ for each $\mathfrak{a} \in \mathcal{I}$. Recall that we have the natural isomorphism $\operatorname{Gr}_{\mathfrak{a}}^{m}\left(E^{\prime}\right)_{\mid \widehat{D}} \simeq \widehat{E}_{\mathfrak{a}}^{\prime}$. Hence, we have the sublattice of $\operatorname{Gr}_{\mathfrak{a}}^{\boldsymbol{m}}\left(E^{\prime}\right)$ corresponding to $\widehat{E}_{\mathfrak{a}} \subset \widehat{E}_{\mathfrak{a}}^{\prime}$, which is denoted by $\operatorname{Gr}_{\mathfrak{a}}^{m}(E)$. It is equipped with a meromorphic flat connection $\nabla_{\mathfrak{a}}$. By construction, we have the isomorphism

$$
\left(\operatorname{Gr}_{\mathfrak{a}}^{m}(E), \nabla_{\mathfrak{a}}\right)_{\mid \widehat{D}} \simeq\left(\widehat{E}_{\mathfrak{a}}, \widehat{\nabla}_{\mathfrak{a}}\right) .
$$

Lemma 5.7. Let $\left(E_{i}, \nabla_{i}\right)(i=1,2)$ be pseudo-good lattices on the level $(\boldsymbol{m}, i(0))$. Let $F:\left(E_{1}, \nabla_{1}\right) \rightarrow\left(E_{2}, \nabla_{2}\right)$ be a flat morphism. Assume $\mathcal{I}_{1} \oplus \mathcal{I}_{2}$ is a good set of irregular values on the level $(\boldsymbol{m}, i(0))$. We have the naturally induced morphism $\operatorname{Gr}_{\mathfrak{a}}^{\boldsymbol{m}}(F): \operatorname{Gr}_{\mathfrak{a}}^{\boldsymbol{m}}\left(E_{1}\right) \rightarrow \operatorname{Gr}_{\mathfrak{a}}^{\boldsymbol{m}}\left(E_{2}\right)$.

Proof. We can take good lattices $\left(E_{i}^{\prime}, \nabla_{i}\right)$ on the level $(\boldsymbol{m}, i(0))$ such that $E_{i} \subset$ $E_{i}^{\prime}$ and $F\left(E_{1}^{\prime}\right) \subset E_{2}^{\prime}$. By Lemma 5.4 we have the induced morphism $\operatorname{Gr}_{\mathfrak{a}}^{m}(F)$ : $\mathrm{Gr}_{\mathfrak{a}}^{\boldsymbol{m}}\left(E_{1}^{\prime}\right) \rightarrow \operatorname{Gr}_{\mathfrak{a}}^{\boldsymbol{m}}\left(E_{2}^{\prime}\right)$. By considering the completion, it is easy to observe that a morphism $\operatorname{Gr}_{\mathfrak{a}}^{\boldsymbol{m}}\left(E_{1}\right) \rightarrow \operatorname{Gr}_{\mathfrak{a}}^{\boldsymbol{m}}\left(E_{2}\right)$ is induced.

Flat splitting and Stokes filtration. Let $\pi: \tilde{X}(D) \rightarrow X$ be the real blow up. Let $S \in \mathcal{M S}^{*}(X-D, \mathcal{I})$. Let $\bar{S}$ denote the closure of $S$ in $\widetilde{X}(D)$, and let $Z$ denote $\bar{S} \cap \pi^{-1}(D)$. We have the Stokes filtration $\mathcal{F}^{S}$ of $E_{\mid \bar{S}}^{\prime}$, and we can take a flat splitting $E_{\mid \bar{S}}^{\prime}=\bigoplus E_{\mathfrak{a}, S}^{\prime}$ such that $E_{\mathfrak{a}, S \mid \widehat{Z}}^{\prime}=\pi^{-1}\left(\widehat{E}_{\mathfrak{a}}^{\prime}\right)$. Because $E_{\mid X-D}=E_{\mid X-D}^{\prime}$, it induces a flat decomposition of $E_{\mid S}$.

Lemma 5.8. The above decomposition extends to a decomposition $E_{\mid \bar{S}}=\bigoplus E_{\mathfrak{a}, S}$ such that $E_{\mathfrak{a}, S \mid \widehat{Z}}=\pi^{-1}\left(\widehat{E}_{\mathfrak{a}}\right)$.

Proof. Let $\boldsymbol{w}_{\mathfrak{a}}$ and $\boldsymbol{w}_{\mathfrak{a}}^{\prime}$ be frames of $\mathrm{Gr}_{\mathfrak{a}}^{\boldsymbol{m}}(E)$ and $\operatorname{Gr}_{\mathfrak{a}}^{\boldsymbol{m}}\left(E^{\prime}\right)$. Let $G_{\mathfrak{a}}$ be determined by $\boldsymbol{w}_{\mathfrak{a}}=\boldsymbol{w}_{\mathfrak{a}}^{\prime} \cdot G_{\mathfrak{a}}$. They induce frames $\widehat{\boldsymbol{w}}_{\mathfrak{a}}$ and $\widehat{\boldsymbol{w}}_{\mathfrak{a}}^{\prime}$ of $\widehat{E}_{\mathfrak{a}}$ and $\widehat{E}_{\mathfrak{a}}^{\prime}$, respectively.

By the isomorphism $E_{\mathfrak{a}, S}^{\prime} \simeq \operatorname{Gr}_{\mathfrak{a}}^{\boldsymbol{m}}\left(E^{\prime}\right)_{\mid \bar{S}}$, we obtain frames $\boldsymbol{w}_{\mathfrak{a}, S}^{\prime}$ of $E_{\mathfrak{a}, S}^{\prime}$. Then $\boldsymbol{w}_{\mathfrak{a}, S}:=\boldsymbol{w}_{\mathfrak{a}, S}^{\prime} \cdot G_{\mathfrak{a}}$ gives a tuple of sections of $E_{\mathfrak{a}, S}^{\prime}$, and we can observe that $\boldsymbol{w}_{\mathfrak{a} \mid \widehat{Z}}=\pi^{-1}\left(\widehat{\boldsymbol{w}}_{\mathfrak{a}}\right)$. Let $E_{\mathfrak{a}, S}$ be generated by $\boldsymbol{w}_{\mathfrak{a}, S}$; thus we obtain the desired decomposition $E=\bigoplus E_{\mathfrak{a}, S}$.

Let $\boldsymbol{w}_{S}=\left(\boldsymbol{w}_{\mathfrak{a}, S}\right)$ be as above. Let $\boldsymbol{v}$ be a frame of $E$ on $X$. Let $G_{S}$ be determined by $\boldsymbol{v}_{\mid S}=\boldsymbol{w}_{S} \cdot G_{S}$. Both $\boldsymbol{v}_{\mid \widehat{Z}}$ and $\boldsymbol{w}_{S \mid \widehat{Z}}$ give the frame of $E_{\mid \widehat{Z}}$, and we obtain the following.

Proposition 5.5. $G_{S}$ and $G_{S}^{-1}$ are bounded on $S$.

Proposition 5.6. The flat subbundles $\mathcal{F}_{\mathfrak{a}}^{S}\left(E_{\mid \bar{S}}\right):=\bigoplus_{\mathfrak{b}<_{S} \mathfrak{a}} \bar{E}_{\mathfrak{b}, S}$ are independent of the choice of a flat decomposition $E_{\mid \bar{S}}=\bigoplus_{\mathfrak{a} \in \mathcal{I}} \bar{E}_{\mathfrak{a}, S}$ such that $\bar{E}_{\mathfrak{a}, S \mid \widehat{Z}}=\pi^{-1} \widehat{E}_{\mathfrak{a}}$. 
Proof. Let $E_{\mid \bar{S}}=\bigoplus_{\mathfrak{a} \in \mathcal{I}} \bar{E}_{\mathfrak{a}, S}$ be another flat decomposition such that $\bar{E}_{\mathfrak{a}, S \mid \widehat{Z}}=$ $\pi^{-1} \widehat{E}_{\mathfrak{a}}$. We take a frame $\overline{\boldsymbol{w}}_{\mathfrak{a}, S}$ of $\bar{E}_{\mathfrak{a}, S}$ such that $\overline{\boldsymbol{w}}_{\mathfrak{a}, S \mid \widehat{Z}}=\widehat{\boldsymbol{w}}_{\mathfrak{a}}$. We set $\overline{\boldsymbol{w}}_{\mathfrak{a}}^{\prime}:=$ $\overline{\boldsymbol{w}}_{\mathfrak{a}} \cdot G_{\mathfrak{a}}^{-1}$. Then $\overline{\boldsymbol{w}}_{\mathfrak{a} \mid \widehat{Z}}^{\prime}=\pi^{-1} \widehat{\boldsymbol{w}}_{\mathfrak{a}}^{\prime}$. Let $\bar{E}_{\mathfrak{a}}^{\prime}$ be generated by $\overline{\boldsymbol{w}}_{\mathfrak{a}}^{\prime}$. Then we obtain a flat decomposition $E_{\mid \bar{S}}^{\prime}=\bigoplus \bar{E}_{\mathfrak{a}}^{\prime}$, which has to be a splitting of the Stokes filtration $\mathcal{F}^{S}\left(E_{\mid \bar{S}}^{\prime}\right)$. Because $\bar{E}_{\mathfrak{a} \mid S}^{\prime}=\bar{E}_{\mathfrak{a} \mid S}$, the filtration is well defined.

Thus, we obtain the filtration $\mathcal{F}^{S}$ of $E_{\mid \bar{S}}$, which is called the Stokes filtration.

Lemma 5.9. We have a natural isomorphism $\operatorname{Gr}_{\mathfrak{a}}^{\mathcal{F}^{S}}\left(E_{\mid \bar{S}}\right) \simeq \operatorname{Gr}_{\mathfrak{a}}(E)_{\mid \bar{S}}$.

Proof. We use the notation in the proof of Lemma 5.8. By the comparison of $\boldsymbol{w}_{\mathfrak{a}}$ and $\boldsymbol{w}_{\mathfrak{a}, S}$, we obtain $E_{\mathfrak{a}, S} \simeq \operatorname{Gr}_{\mathfrak{a}}(E)_{\mid \bar{S}}$. By the construction of the Stokes filtration, we have a natural isomorphism $\operatorname{Gr}_{\mathfrak{a}}^{\mathcal{F}^{S}}\left(E_{\mid \bar{S}}\right) \simeq E_{\mathfrak{a}, S}$. Thus, the claim is clear.

5.1.4. A comparison. Let $Y$ be a complex manifold. Let $X:=\Delta_{z}^{k} \times Y, D_{z, i}:=$ $\left\{z_{i}=0\right\}$ and $D:=\bigcup_{i=1}^{k} D_{z, i}$. Let $\widetilde{\mathcal{K}}$ be a compact region in $\mathbb{C}_{z_{k+1}}$. We set $\widetilde{\mathcal{X}}:=$ $\widetilde{\mathcal{K}} \times X$. We use the symbol $\widetilde{\mathcal{D}}$ in a similar meaning. We set $\widetilde{W}:=\widetilde{\mathcal{D}} \cup(\{0\} \times X)$.

Let $\mathcal{I} \subset M(X, D)$ be a good set of irregular values on the level $(\boldsymbol{m}, i(0))$. We set $\widetilde{\boldsymbol{m}}:=(\boldsymbol{m},-1) \in \mathbb{Z}_{<0}^{k+1}$. We put $\widetilde{\mathfrak{a}}:=z_{k+1}^{-1} \mathfrak{a}$ for $\mathfrak{a} \in \mathcal{I}$, and we set

$$
\widetilde{\mathcal{I}}:=\{\widetilde{\mathfrak{a}} \mid \mathfrak{a} \in \mathcal{I}\} \subset M(\widetilde{\mathcal{X}}, \widetilde{W}) .
$$

This is a good set of irregular values on the level $(\widetilde{\boldsymbol{m}}, i(0))$.

Let $\widetilde{E}$ be a holomorphic vector bundle on $\widetilde{\mathcal{X}}$ with a meromorphic flat connection $\nabla: \widetilde{E} \rightarrow \widetilde{E} \otimes \Omega_{\widetilde{\mathcal{X}}}^{1}(\widetilde{W})$ such that $(\widetilde{E}, \nabla)$ is an unramifiedly good lattice on the level $(\widetilde{\boldsymbol{m}}, i(0))$ on $(\widetilde{\mathcal{X}}, \widetilde{W})$ with the irregular decomposition

$$
(\widetilde{E}, \nabla)_{\mid \widehat{\widetilde{W}}}=\bigoplus_{\widetilde{\mathfrak{a}} \in \widetilde{\mathcal{I}}}\left(\widehat{\widetilde{E}}_{\widetilde{\mathfrak{a}}}, \widehat{\nabla}_{\widetilde{\mathfrak{a}}}\right) .
$$

Applying the general theory of Subsection 5.1.3, we obtain a holomorphic vector bundle $\operatorname{Gr}_{\widetilde{a}}^{\widetilde{m}}(\widetilde{E})$ on $\widetilde{\mathcal{X}}$ with the induced meromorphic flat connection $\nabla_{\widetilde{a}}$ for each $\widetilde{\mathfrak{a}} \in \widetilde{\mathcal{I}}$.

By setting $\lambda=z_{k+1}$, we obtain the isomorphism $\mathbb{C}_{z_{k+1}} \simeq \mathbb{C}_{\lambda}$. Let $\mathcal{K} \subset \mathbb{C}_{\lambda}$ be the image of $\widetilde{\mathcal{K}}$. We put $\mathcal{X}:=\mathcal{K} \times X$ and we use the symbol $\mathcal{D}$ in a similar meaning. We set $W:=\mathcal{D} \cup(\{0\} \times X)$. We have the natural isomorphism $\iota:(\mathcal{X}, \mathcal{D}) \rightarrow(\widetilde{\mathcal{X}}, \widetilde{\mathcal{D}})$. The pull back of $\widetilde{E}$ is denoted by $E$. Let $\mathbb{D}^{f}$ denote the restriction of $\iota^{*} \nabla$ to the $X$-direction. We set $\mathbb{D}:=\lambda \cdot \mathbb{D}^{f}$. Note the following:

- $\mathbb{D}(E) \subset E \otimes p_{\lambda}^{*} \Omega_{X}^{1}(* D)$, i.e., $\mathbb{D}$ gives a family of meromorphic $\lambda$-connections on $E$. 
- $(E, \mathbb{D})$ is a good lattice on the level $(\boldsymbol{m}, i(0))$ on $(\mathcal{X}, W)$, and 5.9 naturally induces the irregular decomposition of $(E, \mathbb{D})_{\mid \widehat{W}}$.

By applying the general theory explained in Subsection 5.1.2, for each $\mathfrak{a} \in \mathcal{I}$, we obtain $\operatorname{Gr}_{\mathfrak{a}}^{\boldsymbol{m}}(E, \mathbb{D})$.

Let $\widetilde{S}$ be a small sector in $\widetilde{\mathcal{X}}-\widetilde{W}$. We have the Stokes filtration $\mathcal{F}^{\widetilde{S}}$ of $\widetilde{E} \mid \overline{\widetilde{S}}$ on the level $\widetilde{\boldsymbol{m}}$ indexed by $\left(\widetilde{\mathcal{I}}, \leq_{\widetilde{S}}\right.$ ) (Proposition 5.6. For $S:=\iota^{-1}(\widetilde{S})$, we have the Stokes filtration $\mathcal{F}^{S}$ of $E_{\mid \bar{S}}$ on the level $\boldsymbol{m}$ indexed by $\left(\mathcal{I}, \leq_{S}\right)$. We remark the following.

Lemma 5.10. Under the natural identification $\widetilde{\mathcal{I}}=\mathcal{I}$, the orders $\leq_{\widetilde{S}}$ and $\leq_{S}$ are the same. Under the natural isomorphism $E \simeq \iota^{*} \widetilde{E}$, the filtrations $\mathcal{F}^{S}$ and $\mathcal{F}^{\widetilde{S}}$ are the same.

Proof. For the order $\leq_{\widetilde{S}}$, we use the identification $\widetilde{\mathcal{X}}=\{1\} \times \widetilde{\mathcal{X}} \subset \mathbb{C}_{\lambda} \times \widetilde{\mathcal{X}}$. Thus, the first claim is clear. Note that both $\iota^{*} \widetilde{\mathcal{F}}^{\widetilde{S}}$ and $\mathcal{F}^{S}$ satisfy the condition in Proposition 5.1. Hence, they are the same.

Corollary 5.1. We have a natural isomorphism $\iota^{*} \operatorname{Gr}_{\widetilde{\mathfrak{a}}}^{\widetilde{m}}(\widetilde{E}) \simeq \operatorname{Gr}_{\mathfrak{a}}^{\boldsymbol{m}}(E)$, and $\mathbb{D}_{\mathfrak{a}}$ is induced by $\iota^{*} \nabla_{\widetilde{a}}$ via the above procedure.

Proof. Lemma 5.10, yields the isomorphism $j: \iota^{*} \operatorname{Gr}_{\widetilde{\mathfrak{a}}}^{\widetilde{m}}(\widetilde{E})_{\mid \mathcal{X}-W} \simeq \operatorname{Gr}_{\mathfrak{a}}^{m}(E)_{\mid \mathcal{X}-W}$, on which $\mathbb{D}_{\mathfrak{a}}$ is induced by $\nabla_{\mathfrak{a}}$ via the above procedure. Since $j$ extends on $\widetilde{\mathcal{X}}(W)$, it extends on $\mathcal{X}$.

5.1.5. Stokes filtration of the associated flat bundle on the real blow up. We use the setting in Subsection 5.1.3. Let $\mathcal{I} \subset M(X, D)$ be a good set of irregular values on the level $(\boldsymbol{m}, i(0))$. Let $E$ be a holomorphic vector bundle on $X$ with a meromorphic flat connection $\nabla: E \rightarrow E \otimes \Omega_{X}^{1}(* D)$ such that $(E, \nabla)$ is a pseudo-good lattice on the level $(\boldsymbol{m}, i(0))$. (In other words, we consider a family of meromorphic $\lambda$-flat bundles on $\{1\} \times(X, D)$.) Let $\pi: \widetilde{X}(D) \rightarrow X$ be a real blow up of $X$ along $D$. The flat bundle $E_{\mid X-D}$ naturally extends to a flat bundle $\mathfrak{V}$ on $\widetilde{X}(D)$.

We set $\mathfrak{Z}:=\pi^{-1}\left(O_{z} \times Y\right)$. For each $P \in \mathfrak{Z}$, we take a small sector $S \in$ $\mathcal{M S}(P, X-D, \mathcal{I})$ on which we have the Stokes filtration $\mathcal{F}^{S}$ of $E_{\mid S}$. The filtration naturally extends to a flat filtration of $\mathfrak{V}_{\mid \bar{S}}$. By restricting it to the fiber $\mathfrak{V}_{\mid P}$, we obtain a filtration $\mathcal{F}^{P}$ indexed by $\left(\mathcal{I}, \leq_{P}\right)$. It is easy to observe that $\mathcal{F}^{P}$ is well defined.

If $Q \in \pi^{-1}(\mathfrak{Z})$ is sufficiently close to $P$, the map $\left(\mathcal{I}, \leq_{P}\right) \rightarrow\left(\mathcal{I}, \leq_{Q}\right)$ preserves the orders, and the filtrations $\mathcal{F}^{P}$ and $\mathcal{F}^{Q}$ are compatible under the identification $\mathfrak{V}_{\mid P} \simeq \mathfrak{V}_{\mid Q}$ given by the parallel transport in $\bar{S}_{P}$. In particular, we have $\mathcal{F}^{P}=\mathcal{F}^{Q}$ 
if $\leq_{P}=\leq_{Q}$. We have the functoriality of the filtrations $\mathcal{F}^{P}$ for dual, tensor product and direct sum as in the case of $\mathcal{F}^{S}$. We also have the functoriality for morphisms as follows.

Lemma 5.11. Let $F:\left(E_{1}, \nabla_{1}\right) \rightarrow\left(E_{2}, \nabla_{2}\right)$ be a flat morphism. For simplicity, assume that $\mathcal{I}_{1} \cup \mathcal{I}_{2}$ is a good set of irregular values on the level $(\boldsymbol{m}, i(0))$. The induced morphism $F_{\mid P}: \mathfrak{V}_{1 \mid P} \rightarrow \mathfrak{V}_{2 \mid P}$ preserves the Stokes filtrations $\mathcal{F}^{P}$.

Remark 13. We considered two vector bundles on $\widetilde{X}(D)$. One is $\pi^{-1}(E)$ and the other is $\mathfrak{V}$. We should emphasize that they are different in general. The bundle $\mathfrak{V}$ depends only on the flat bundle $(E, \nabla)_{\mid X-D}$, and $\pi^{-1}(E)$ depends on the prolongment $(E, \nabla)$.

Let us look at the simplest example $E=\mathcal{O} \cdot e$ with $\nabla(e)=e \cdot d\left(z^{-1}\right)$. A trivialization of $\pi^{-1}(E)$ is given by $\pi^{-1}(e)$. A trivialization of $\mathfrak{V}$ is induced by $\exp \left(-z^{-1}\right) \cdot e$.

\section{§5.2. Unramifiedly good lattices of a family of meromorphic $\lambda$-flat bundles}

\subsubsection{Preliminaries}

Good set of irregular values. We use the partial order $\leq_{\mathbb{Z}^{n}}$ on $\mathbb{Z}^{n}$ given by $\boldsymbol{a} \leq_{\mathbb{Z}^{n}} \boldsymbol{b} \Leftrightarrow a_{i} \leq b_{i}(\forall i)$. We write $\boldsymbol{a}<\mathbb{Z}^{n} \boldsymbol{b}$ if $a_{i}<b_{i}$ for any $i$, and $\boldsymbol{a} \succ_{\mathbb{Z}^{n}} \boldsymbol{b}$ if $\boldsymbol{a} \leq_{\mathbb{Z}^{n}} \boldsymbol{b}$ and $\boldsymbol{a} \neq \boldsymbol{b}$. Let $\boldsymbol{\delta}_{j}$ denote the element $\overbrace{0, \ldots, 0}^{j-1}, 1,0, \ldots, 0)$, and let $\mathbf{0}$ denote the zero in $\mathbb{Z}^{n}$. We also use $\mathbf{0}_{n}$ when we wish to indicate the dependence on $n$. For a positive integer $\ell$, we put $\underline{\ell}:=\{1, \ldots, \ell\}$.

Let $Y$ be a complex manifold. Put $X:=\Delta^{\ell} \times Y$. Let $D_{i}:=\left\{z_{i}=0\right\} \times Y$ and $D:=\bigcup_{i=1}^{\ell} D_{i}$ be the hypersurfaces of $X$. We also put $D_{\underline{\ell}}=\bigcap_{i=1}^{\ell} D_{i}$, which is naturally identified with $Y$.

For any $f \in M(X, D)$, we have the Laurent expansion

$$
f=\sum_{\boldsymbol{m} \in \mathbb{Z}^{\ell}} f_{\boldsymbol{m}}(y) \boldsymbol{z}^{\boldsymbol{m}} .
$$

Here $f_{\boldsymbol{m}}$ are holomorphic functions on $D_{\underline{\ell}}$. We often use the following identification implicitly:

$$
M(X, D) / \boldsymbol{z}^{\boldsymbol{n}} H(X) \simeq\left\{f \in M(X, D) \mid f_{\boldsymbol{m}}=0, \forall \boldsymbol{m} \geq \boldsymbol{n}\right\} .
$$

For any $f \in M(X, D)$, let ord $(f)$ denote the minimum of the set $\left\{\boldsymbol{m} \in \mathbb{Z}^{\ell} \mid\right.$ $\left.f_{\boldsymbol{m}} \neq 0\right\} \cup\{\mathbf{0}\}$ with respect to $\leq_{\mathbb{Z}^{\ell}}$, if it exists. It is always contained in $\mathbb{Z}_{\leq 0}^{\ell}$, if it exists. 
For any $\mathfrak{a} \in M(X, D) / H(X)$, we take any lift $\tilde{\mathfrak{a}}$ to $M(X, D)$, and we set $\operatorname{ord}(\mathfrak{a}):=\operatorname{ord}(\widetilde{\mathfrak{a}})$ if the right hand side exists. If $\operatorname{ord}(\mathfrak{a})$ exists in $\mathbb{Z}_{\leq 0}^{\ell} \backslash\{\mathbf{0}\}, \widetilde{\mathfrak{a}}_{\text {ord }(\mathfrak{a})}$ is independent of the choice of a lift $\widetilde{\mathfrak{a}}$, and is denoted by $\mathfrak{a}_{\operatorname{ord}(\mathfrak{a})}$.

Definition 5.3. A finite subset $\mathcal{I} \subset M(X, D) / H(X)$ is called a good set of irregular values on $(X, D)$ if the following conditions are satisfied:

- $\operatorname{ord}(\mathfrak{a})$ exists for each $\mathfrak{a} \in \mathcal{I}$, and $\mathfrak{a}_{\text {ord }(\mathfrak{a})}$ is nowhere vanishing on $D_{\underline{\ell}}$ for $\mathfrak{a} \neq 0$.

- For any two distinct $\mathfrak{a}, \mathfrak{b} \in \mathcal{I}$, ord $(\mathfrak{a}-\mathfrak{b})$ exists in $\mathbb{Z}_{\leq 0}^{\ell} \backslash\{\mathbf{0}\}$, and $(\mathfrak{a}-\mathfrak{b})_{\operatorname{ord}(\mathfrak{a}-\mathfrak{b})}$ is nowhere vanishing on $D_{\underline{\ell}}$.

- The set $\mathcal{T}(\mathcal{I}):=\{\operatorname{ord}(\mathfrak{a}-\mathfrak{b}) \mid \mathfrak{a}, \mathfrak{b} \in \mathcal{I}\}$ is totally ordered with respect to the partial order on $\mathbb{Z}^{\ell}$.

The condition in Definition 5.3 does not depend on the choice of a holomorphic coordinate such that $D=\bigcup_{i=1}^{\ell}\left\{z_{i}=0\right\}$.

Remark 14. The third condition in Definition 5.3 is stronger than that in 24 . It is a little more convenient for our inductive argument. However, it is not essential, because such conditions can be satisfied after birational transformation, once we have decompositions as in 5.12 .

We will use the following lemma implicitly.

Lemma 5.12. The set $\{\operatorname{ord}(\mathfrak{a}) \mid \mathfrak{a} \in \mathcal{I}\}$ is totally ordered. In particular, the minimum

$$
\boldsymbol{m}(0):=\min \{\operatorname{ord}(\mathfrak{a}) \mid \mathfrak{a} \in \mathcal{I}\}
$$

exists. Moreover, $\boldsymbol{m}(0) \leq_{\mathbb{Z}^{\ell}} \boldsymbol{m}$ for any $\boldsymbol{m} \in \mathcal{T}(\mathcal{I})$.

Proof. Let $\mathfrak{a}, \mathfrak{b} \in \mathcal{I}$. Assume ord $(\mathfrak{a}) \not \subset \operatorname{ord}(\mathfrak{b})$ and $\operatorname{ord}(\mathfrak{a}) \nsupseteq \operatorname{ord}(\mathfrak{b})$. Then $\operatorname{ord}(\mathfrak{a}-\mathfrak{b})$ does not exist, which contradicts the second condition of Definition 5.3. Hence, we obtain the first claim of the lemma. For any $\boldsymbol{m} \in \mathcal{T}(\mathcal{I})$, there exists $\mathfrak{a} \in \mathcal{I}$ such that $\mathfrak{a}_{\boldsymbol{m}} \neq 0$. Hence, $\boldsymbol{m}(0) \leq_{\mathbb{Z}^{\ell}} \boldsymbol{m}$.

Remark 15. It is often convenient to use a coordinate such that $\mathcal{T}(\mathcal{I}) \cup\{\boldsymbol{m}(0)\} \subset$ $\coprod_{i=0}^{\ell} \mathbb{Z}_{<0}^{i} \times \mathbf{0}_{\ell-i}$.

Auxiliary sequence. Let $\mathcal{I}$ be a good set of irregular values on $(X, D)$. Since the set $\mathcal{T}(\mathcal{I})$ is totally ordered by $\leq_{\mathbb{Z}^{\ell}}$, we can take a sequence

$$
\mathcal{M}:=(\boldsymbol{m}(0), \boldsymbol{m}(1), \ldots, \boldsymbol{m}(L), \boldsymbol{m}(L+1)) \subset \mathbb{Z}_{\leq 0}^{\ell}
$$

with the following property:

- $\mathcal{T}(\mathcal{I}) \subset \mathcal{M}$ and $\boldsymbol{m}(L+1)=\mathbf{0}_{\ell}$.

- We have $1 \leq \mathfrak{h}(i) \leq \ell$ such that $\boldsymbol{m}(i+1)=\boldsymbol{m}(i)+\boldsymbol{\delta}_{\mathfrak{h}(i)}$ for each $i \leq L$. 
Such a sequence is called an auxiliary sequence for $\mathcal{I}$. It is not uniquely determined by $\mathcal{I}$. It is convenient for an inductive argument.

Truncation. Let $\mathcal{I}$ be a good set of irregular values. We take an auxiliary sequence for $\mathcal{I}$, and let $\bar{\eta}_{\boldsymbol{m}(0)}: \mathcal{I} \rightarrow M(X, D) / H(X)$ be given as follows:

$$
\bar{\eta}_{\boldsymbol{m}(0)}(\mathfrak{a}):=\sum_{n \nsupseteq m(1)} \mathfrak{a}_{n} z^{n}
$$

Its image is a good set of irregular values on the level $(\boldsymbol{m}(0), i(0))$. More generally, $\bar{\eta}_{\boldsymbol{m}(j)}$ is defined as follows:

$$
\bar{\eta}_{\boldsymbol{m}(j)}(\mathfrak{a}):=\sum_{\boldsymbol{n} \nsupseteq \boldsymbol{m}(j+1)} \mathfrak{a}_{\boldsymbol{n}} \boldsymbol{z}^{\boldsymbol{n}} .
$$

We have $\bar{\eta}_{\boldsymbol{m}(L)}(\mathfrak{a})=\mathfrak{a}$. We set $\zeta_{\boldsymbol{m}(0)}(\mathfrak{a}):=\bar{\eta}_{\boldsymbol{m}(0)}(\mathfrak{a})$ and $\zeta_{\boldsymbol{m}(j)}(\mathfrak{a}):=\bar{\eta}_{\boldsymbol{m}(j)}(\mathfrak{a})$ $-\bar{\eta}_{\boldsymbol{m}(j-1)}(\mathfrak{a})$ for $j=1, \ldots, L$. Then we have the decomposition $\bar{\eta}_{\boldsymbol{m}(i)}(\mathfrak{a})=$ $\sum_{j \leq i} \zeta_{\boldsymbol{m}(j)}(\mathfrak{a})$.

Let $\mathcal{I}(\boldsymbol{m}(i))$ denote the image of $\bar{\eta}_{\boldsymbol{m}(i)}: \mathcal{I} \rightarrow M(X, D) / H(X)$.

Lemma 5.13. If we shrink $X$ appropriately, $\mathcal{I}(\boldsymbol{m}(0))$ is a good set of irregular values on the level $(\boldsymbol{m}(0), \mathfrak{h}(0))$.

Proof. If $\bar{\eta}_{\boldsymbol{m}(0)}(\mathfrak{a}-\mathfrak{b}) \neq 0$ for $\mathfrak{a}, \mathfrak{b} \in \mathcal{I}$, then we have ord $(\mathfrak{a}-\mathfrak{b})=\boldsymbol{m}(0)$ and $\left(\boldsymbol{z}^{-\boldsymbol{m}(0)} \bar{\eta}_{\boldsymbol{m}(0)}(\mathfrak{a}-\mathfrak{b})\right)_{\mid D_{\underline{\ell}}}$ is nowhere vanishing, so $\boldsymbol{z}^{-\boldsymbol{m}(0)} \bar{\eta}_{\boldsymbol{m}(0)}(\mathfrak{a}-\mathfrak{b})$ is nowhere vanishing on $X$ provided $X$ is shrinked appropriately. Similarly, $\left(\boldsymbol{z}^{-\boldsymbol{m}(0)} \bar{\eta}_{\boldsymbol{m}(0)}(\mathfrak{a})\right)$ may be nowhere vanishing on $X$ provided $X$ is shrinked appropriately.

We can use the following lemma for inductive arguments.

Lemma 5.14. For any $\mathfrak{b} \in \mathcal{I}(\boldsymbol{m}(0))$, fix any element $\mathfrak{a}^{(0)} \in \bar{\eta}_{\boldsymbol{m}(0)}^{-1}(\mathfrak{b})$. Then the set

$$
\left\{\mathfrak{a}-\mathfrak{a}^{(0)} \mid \bar{\eta}_{\boldsymbol{m}(0)}(\mathfrak{a})=\mathfrak{b}\right\}
$$

is also a good set of irregular values.

Example. We give some examples. Set

$$
\mathfrak{a}^{(1)}:=z_{1}^{-1} z_{2}^{-1}, \quad \mathfrak{a}^{(2)}:=z_{1}^{-1}, \quad \mathfrak{a}^{(3)}:=0 .
$$

An auxiliary sequence is unique in this case, and given as follows:

$$
\boldsymbol{m}(0)=(-1,-1), \mathfrak{h}(0)=2, \quad \boldsymbol{m}(1)=(-1,0), \mathfrak{h}(1)=1, \quad \boldsymbol{m}(2)=(0,0)
$$


The truncations are given as follows:

$$
\begin{aligned}
& \bar{\eta}_{\boldsymbol{m}(0)}\left(\mathfrak{a}^{(1)}\right)=\mathfrak{a}^{(1)}, \quad \bar{\eta}_{\boldsymbol{m}(0)}\left(\mathfrak{a}^{(2)}\right)=0, \quad \bar{\eta}_{\boldsymbol{m}(0)}\left(\mathfrak{a}^{(3)}\right)=0, \\
& \bar{\eta}_{\boldsymbol{m}(1)}\left(\mathfrak{a}^{(1)}\right)=\mathfrak{a}^{(1)}, \quad \bar{\eta}_{\boldsymbol{m}(1)}\left(\mathfrak{a}^{(2)}\right)=\mathfrak{a}^{(2)}, \quad \bar{\eta}_{\boldsymbol{m}(1)}\left(\mathfrak{a}^{(3)}\right)=\mathfrak{a}^{(3)} .
\end{aligned}
$$

The image of $\mathcal{I}$ via $\bar{\eta}_{\boldsymbol{m}(0)}$ is $\left\{\mathfrak{a}^{(1)}, 0\right\}$.

Let us consider the set which consists of the following:

$$
\mathfrak{b}^{(1)}=z_{1}^{-1} z_{2}^{-1}+a z_{2}^{-1}+b z_{1}^{-1}, \quad \mathfrak{b}^{(2)}=z_{1}^{-1} .
$$

An auxiliary sequence is given by (5.11). The truncation is given as follows:

$$
\bar{\eta}_{\boldsymbol{m}(0)}\left(\mathfrak{b}^{(1)}\right)=z_{1}^{-1} z_{2}^{-1}+a z_{2}^{-1}, \quad \bar{\eta}_{\boldsymbol{m}(0)}\left(\mathfrak{b}^{(2)}\right)=0 .
$$

We have the following pictures in mind for truncation:
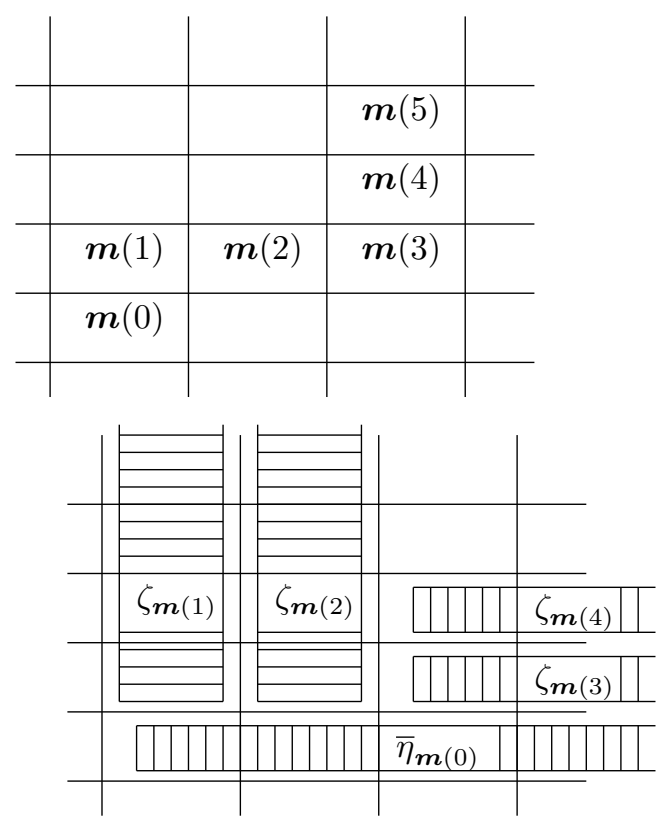

$$
\begin{aligned}
L=4, & \boldsymbol{m}(0)=(-2,-3), \boldsymbol{m}(1)=(-2,-2), \boldsymbol{m}(2)=(-1,-2), \\
\boldsymbol{m}(3) & =(0,-2), \boldsymbol{m}(4)=(0,-1), \boldsymbol{m}(5)=(0,0) .
\end{aligned}
$$

5.2.2. Unramifiedly good lattices of a family of meromorphic $\lambda$-flat bundles. Let $X$ be a complex manifold, and let $D$ be a normal crossing divisor of $X$. Let $\mathcal{K}$ be a point or a compact region in $\mathbb{C}_{\lambda}$. Let $\mathcal{X}$ and $\mathcal{D}$ denote $\mathcal{K} \times X$ and $\mathcal{K} \times D$, respectively. For $\lambda \in \mathcal{K}$, we set $\mathcal{X}^{\lambda}:=\{\lambda\} \times X$ and $\mathcal{D}^{\lambda}:=\{\lambda\} \times D$. Let 
$(\mathcal{E}, \mathbb{D})$ be a family of meromorphic $\lambda$-flat bundles on $(\mathcal{X}, \mathcal{D})$, i.e., $\mathcal{E}$ is an $\mathcal{O}_{\mathcal{X}}(* \mathcal{D})$ coherent sheaf with a holomorphic family of flat $\lambda$-connections $\mathbb{D}: \mathcal{E} \rightarrow \mathcal{E} \otimes \Omega_{\mathcal{X} / \mathcal{K}}^{1}$. The restriction to $\left(\mathcal{X}^{\lambda}, \mathcal{D}^{\lambda}\right)$ is denoted by $\left(\mathcal{E}^{\lambda}, \mathbb{D}^{\lambda}\right)$.

Remark 16. If $\mathcal{K}$ is a point, "family" can be omitted.

Let $E$ be an $\mathcal{O}_{\mathcal{X}}$-locally free lattice of $(\mathcal{E}, \mathbb{D})$. Let $P$ be any point of $\mathcal{D}$. We can take a holomorphic coordinate $\left(\mathcal{U}, \lambda, z_{1}, \ldots, z_{n}\right)$ around $P$ such that $\mathcal{D}_{\mathcal{U}}:=$ $\mathcal{D} \cap \mathcal{U}=\bigcup_{i=1}^{\ell} \mathcal{D}_{\mathcal{U}, i}$, where $\mathcal{D}_{\mathcal{U}, i}:=\left\{z_{i}=0\right\}$. We put $\mathcal{D}_{\mathcal{U}, I}:=\bigcap_{i \in I} \mathcal{D}_{\mathcal{U}, i}$ and $\mathcal{D}_{\mathcal{U}}(I):=\bigcup_{i \in I} \mathcal{D}_{\mathcal{U}, i}$. For any subset $I \subset \underline{\ell}$, we put $I^{c}:=\underline{\ell}-I$. The completion of $\mathcal{X}$ along $\mathcal{D}_{\mathcal{U}, I}$ (resp. $\left.\mathcal{D}_{\mathcal{U}}(I)\right)$ is denoted by $\widehat{\mathcal{D}}_{\mathcal{U}, I}$ (resp. $\widehat{\mathcal{D}}_{\mathcal{U}}(I)$ ).

Definition 5.4. We say that $E$ is unramifiedly good at $P$ if the following holds:

- We are given a good set of irregular values $S \subset M\left(\mathcal{U}, \mathcal{D}_{\mathcal{U}}\right) / H(\mathcal{U})$.

- For any $\emptyset \neq I \subset \underline{\ell}$, we have the decomposition

$$
(E, \mathbb{D})_{\mid \widehat{\mathcal{D}}_{\mathcal{U}, I}}=\bigoplus_{\mathfrak{a} \in S(I)}\left({ }^{I} \widehat{E}_{\mathfrak{a}},{ }^{I} \widehat{\mathbb{D}}_{\mathfrak{a}}\right) .
$$

Here $S(I)$ denotes the image of $S$ via the map

$$
M\left(\mathcal{U}, \mathcal{D}_{\mathcal{U}}\right) / H(\mathcal{U}) \rightarrow M\left(\mathcal{U}, \mathcal{D}_{\mathcal{U}}\right) / M\left(\mathcal{U}, \mathcal{D}_{\mathcal{U}}\left(I^{c}\right)\right) .
$$

- $\left(\mathbb{D}_{\mathfrak{a}}-d \mathfrak{a}\right)\left({ }^{I} \widehat{E}_{\mathfrak{a}}\right)$ is contained in ${ }^{I} \widehat{E}_{\mathfrak{a}} \otimes\left(\Omega_{\mathcal{X} / \mathcal{K}}^{1}\left(\log \mathcal{D}_{\mathcal{U}}(I)\right)+\Omega_{\mathcal{X} / \mathcal{K}}^{1}\left(* \mathcal{D}_{\mathcal{U}}\left(I^{c}\right)\right)\right)$, where $\mathfrak{a}$ is lifted to $M\left(\mathcal{U}, \mathcal{D}_{\mathcal{U}}\right)$. This condition is independent of the choice of a lift.

The property is independent of the choice of the coordinate $\left(\mathcal{U}, \lambda, z_{1}, \ldots, z_{n}\right)$.

We say that $(E, \mathbb{D})$ is unramifiedly good if it is unramifiedly good at any point.

See Subsection 2.3 of [23] for a simplified definition.

The decomposition 5.12 is called the irregular decomposition of $E_{\mid \widehat{\mathcal{D}}_{\mathcal{U}, I}}$. The set $S$ is uniquely determined if $\underline{\ell} E_{\mathfrak{a}} \neq 0$ for each $\mathfrak{a} \in S$. So, it is denoted by $\operatorname{Irr}(\mathbb{D}, P)$. The restriction of $E$ to $\{\lambda\} \times X$ is denoted by $E^{\lambda}$.

If $E$ is an unramifiedly good lattice of $(\mathcal{E}, \mathbb{D})$, we have the well defined endomorphism $\operatorname{Res}_{i}(\mathbb{D})$ of $E_{\mid \mathcal{D}_{i}}$ for each irreducible component $D_{i}$ of $D$. It is called the residue of $\mathbb{D}$ at $D_{i}$ with respect to the lattice $E$. If $\mathcal{K} \neq\{0\}$, the eigenvalues of $\operatorname{Res}_{i}(\mathbb{D})$ are constant on $\mathcal{D}_{i}^{\lambda}$ for each $\lambda \in \mathcal{K}$.

Remark 17. We have the notion of good lattice which is locally a descent of an unramifiedly good lattice. See [23] and Definition 5.5 below. 
Irregular decompositions on the level $\boldsymbol{m}(j)$. In the following, let $X:=\Delta^{n}$, $D_{i}:=\left\{z_{i}=0\right\}$ and $D:=\bigcup_{i=1}^{\ell} D_{i}$. We set $\mathcal{D}(\leq p):=\bigcup_{i \leq p} \mathcal{D}_{i}$. Let $(E, \mathbb{D})$ be an unramifiedly good lattice of a family of meromorphic $\lambda$-flat bundles on $(\mathcal{X}, \mathcal{D})$ with the good set $\operatorname{Irr}(\mathbb{D})=\operatorname{Irr}(\mathbb{D}, O)$. We assume that the coordinate is as in Remark 15 for $\operatorname{Irr}(\mathbb{D})$. Let $\operatorname{Irr}(\mathbb{D}, p)$ and $\operatorname{Irr}^{\prime}(\mathbb{D}, p)$ denote the images of $\operatorname{Irr}(\mathbb{D})$ under the natural maps

$$
\begin{aligned}
& \pi_{p}: M(\mathcal{X}, \mathcal{D}) / H(\mathcal{D}) \rightarrow M(\mathcal{X}, \mathcal{D}) / M(\mathcal{X}, \mathcal{D}(\leq p-1)), \\
& \pi_{p}^{\prime}: M(\mathcal{X}, \mathcal{D}) / H(\mathcal{D}) \rightarrow M(\mathcal{X}, \mathcal{D}) / M(\mathcal{X}, \mathcal{D}(\neq p))
\end{aligned}
$$

Note that the naturally induced map $\operatorname{Irr}(\mathbb{D}, p) \rightarrow \operatorname{Irr}^{\prime}(\mathbb{D}, p)$ is bijective, permitting one to identify them.

Take an auxiliary sequence $\boldsymbol{m}(0), \ldots, \boldsymbol{m}(L)$ for the good set $\operatorname{Irr}(\mathbb{D})$. Denote by $\overline{\operatorname{Irr}}(\mathbb{D}, \boldsymbol{m}(j))$ the image of $\operatorname{Irr}(\mathbb{D})$ under $\bar{\eta}_{\boldsymbol{m}(j)}$. Let $k(j)$ denote the number determined by the condition $\boldsymbol{m}(j) \in \mathbb{Z}_{<0}^{k(j)} \times \mathbf{0}_{\ell-k(j)}$. The map $\operatorname{Irr}(\mathbb{D}, p) \rightarrow$ $M(\mathcal{X}, \mathcal{D}) / M(\mathcal{X}, \mathcal{D}(\leq p-1))$ induced by $\bar{\eta}_{\boldsymbol{m}(j)}$ is denoted by $\bar{\eta}_{\boldsymbol{m}(j), p}$. As in Subsection 2.4 .3 of [23], we obtain the following decomposition on the completion $\widehat{\mathcal{D}}(\leq k(j))$ along $\mathcal{D}(\leq k(j))$ :

$$
\begin{aligned}
(E, \mathbb{D})_{\mid \widehat{\mathcal{D}}(\leq k(j))} & =\bigoplus_{\mathfrak{b} \in \overline{\operatorname{Irr}}(\mathbb{D}, \boldsymbol{m}(j))}\left(\widehat{E}_{\mathfrak{b}}^{\boldsymbol{m}(j)}, \mathbb{D}_{\mathfrak{b}}\right) \quad \text { where } \\
\widehat{E}_{\mathfrak{b} \mid \widehat{\mathcal{D}}_{p}}^{\boldsymbol{m}(j)} & =\bigoplus_{\substack{\mathfrak{c} \in \operatorname{Irr}(\mathbb{D}, p) \\
\bar{\eta}_{\boldsymbol{m}(j), p}(\mathfrak{c})=\pi_{p}(\mathfrak{b})}} \widehat{E}_{\mathfrak{c}} \quad(p \leq k(j)) .
\end{aligned}
$$

The decomposition $(5.13)$ is called the irregular decomposition on the level $\boldsymbol{m}(j)$.

Remark 18. We do not have the irregular decomposition on the level $\boldsymbol{m}(j)$ on $\widehat{D}$ in general, as remarked by Sabbah [24] for the surface case.

The associated graded bundles with a family of meromorphic flat $\lambda$ connections. Assume $\mathcal{K} \neq\{0\}$. We set $W:=\mathcal{X}^{0} \cup \mathcal{D}(\leq k(0))$. It is easy to observe that $(E, \mathbb{D})$ is an unramifiedly good lattice on the level $(\boldsymbol{m}(0), i(0))$ with the decomposition (5.13) for $j=0$. The set of irregular values on the level $(\boldsymbol{m}(0), i(0))$ is $\overline{\operatorname{Irr}}(\mathbb{D}, \boldsymbol{m}(0))$.

As stated in Subsection 5.1.2 we obtain the holomorphic bundle $\operatorname{Gr}_{\mathfrak{a}}^{\boldsymbol{m}(0)}(E)$ with a family of meromorphic flat $\lambda$-connections $\mathbb{D}_{\mathfrak{a}}^{\boldsymbol{m}(0)}$ on $(\mathcal{V}, \mathcal{V} \cap \mathcal{D})$ for each $\mathfrak{a} \in \overline{\operatorname{Irr}}(\mathbb{D}, \boldsymbol{m}(0))$, where $\mathcal{V}$ denotes a neighbourhood of $\bigcap_{1 \leq i \leq k(0)} \mathcal{D}_{i}$. Let $\operatorname{Gr}_{\mathfrak{a}}^{\boldsymbol{m}(0)}(E, \mathbb{D}):=\left(\operatorname{Gr}_{\mathfrak{a}}^{\boldsymbol{m}(0)}(E), \mathbb{D}_{\mathfrak{a}}^{\boldsymbol{m}(0)}\right)$. We obtain the following isomorphisms for any $\mathfrak{a} \in \overline{\operatorname{Irr}}(\mathbb{D}, \boldsymbol{m}(0))$ from 5.8 :

$$
\operatorname{Gr}_{\mathfrak{a}}^{\boldsymbol{m}(0)}(E, \mathbb{D})_{\mid \widehat{W}} \simeq\left(\widehat{E}_{\mathfrak{a}}^{\boldsymbol{m}(0)}, \mathbb{D}_{\mathfrak{a}}\right)
$$


In particular, $\operatorname{Gr}_{\mathfrak{a}}^{\boldsymbol{m}(0)}(E, \mathbb{D})$ are unramifiedly good lattices whose set of irregular values is $\operatorname{Irr}\left(\mathbb{D}_{\mathfrak{a}}^{\boldsymbol{m}(0)}\right)=\bar{\eta}_{\boldsymbol{m}(0)}^{-1}(\mathfrak{a})$.

Let $\overline{\operatorname{Irr}}(\mathbb{D}, \boldsymbol{m}(j))$ denote the image of $\bar{\eta}_{\boldsymbol{m}(j)}: \operatorname{Irr}(\mathbb{D}) \rightarrow M(X, D) / H(X)$ for any $j$. Let us consider the case in which $\overline{\operatorname{Irr}}(\mathbb{D}, \boldsymbol{m}(j-1))$ consists of a unique element. We take any element $\mathfrak{a}^{(1)} \in \operatorname{Irr}(\mathbb{D})$. Let $\mathcal{L}\left( \pm \mathfrak{a}^{(1)}\right)$ be a line bundle $\mathcal{O}_{\mathcal{X}} \cdot e$ with a family of meromorphic flat $\lambda$-connections $\mathbb{D} e=e \cdot\left( \pm d \mathfrak{a}^{(1)}\right)$. Then $\left(E^{\prime}, \mathbb{D}^{\prime}\right):=$ $(E, \mathbb{D}) \otimes \mathcal{L}\left(-\mathfrak{a}^{(1)}\right)$ is an unramifiedly good lattice with the good set

$$
\operatorname{Irr}\left(\mathbb{D}^{\prime}\right)=\left\{\mathfrak{a}-\mathfrak{a}^{(1)} \mid \mathfrak{a} \in \operatorname{Irr}(\mathbb{D})\right\}
$$

The sequence $\boldsymbol{m}(j), \boldsymbol{m}(j+1), \ldots, \boldsymbol{m}(L)$ gives an auxiliary sequence for $\operatorname{Irr}\left(\mathbb{D}^{\prime}\right)$. Applying the above procedure to $\left(E^{\prime}, \mathbb{D}^{\prime}\right)$ and shrinking $X$, we obtain the associated $\operatorname{Gr}_{\mathfrak{c}}^{\boldsymbol{m}(j)}\left(E^{\prime}, \mathbb{D}^{\prime}\right)$ for each $\mathfrak{c} \in \overline{\operatorname{Irr}}\left(\mathbb{D}^{\prime}, \boldsymbol{m}(j)\right)$. For any $\mathfrak{b} \in \overline{\operatorname{Irr}}(\mathbb{D}, \boldsymbol{m}(j))$, we define

$$
\operatorname{Gr}_{\mathfrak{b}}^{\boldsymbol{m}(j)}(E, \mathbb{D}):=\operatorname{Gr}_{\mathfrak{b}-\bar{\eta}_{\boldsymbol{m}(j)}(\mathfrak{a}(1))}^{\boldsymbol{m}(j)}\left(E^{\prime}, \mathbb{D}^{\prime}\right) \otimes \mathcal{L}\left(\mathfrak{a}^{(1)}\right)
$$

It is independent of the choice of $\mathfrak{a}^{(1)}$ up to canonical isomorphisms. (We may avoid tensor products.) It is easy to observe that $\operatorname{Gr}_{\mathfrak{b}}^{\boldsymbol{m}(j)}(E, \mathbb{D})$ are also unramifiedly good lattices with the good sets of irregular values $\operatorname{Irr}\left(\mathbb{D}_{\mathfrak{b}}^{\boldsymbol{m}(j)}\right)=\bar{\eta}_{\boldsymbol{m}(j)}^{-1}(\mathfrak{b})$. By construction, $\overline{\operatorname{Irr}}\left(\mathbb{D}_{\mathfrak{b}}^{\boldsymbol{m}(j)}, \boldsymbol{m}(j)\right)$ consists of the unique element $\mathfrak{b}$.

In the general case, let $\bar{\eta}_{\boldsymbol{m}(j-1), \boldsymbol{m}(j)}: \overline{\operatorname{Irr}}(\mathbb{D}, \boldsymbol{m}(j)) \rightarrow \overline{\operatorname{Irr}}(\mathbb{D}, \boldsymbol{m}(j-1))$ be the induced map. For any $\mathfrak{a} \in \overline{\operatorname{Irr}}(\mathbb{D}, \boldsymbol{m}(j))$, we inductively define

$$
\operatorname{Gr}_{\mathfrak{a}}^{\boldsymbol{m}(j)}(E, \mathbb{D}):=\operatorname{Gr}_{\mathfrak{a}}^{\boldsymbol{m}(j)} \operatorname{Gr}_{\bar{\eta}_{\boldsymbol{m}(j-1), \boldsymbol{m}(j)}^{\boldsymbol{m}(j)}}^{(\mathfrak{a})}(E, \mathbb{D})
$$

For each $\mathfrak{a} \in \operatorname{Irr}(\mathbb{D})$, we set $\operatorname{Gr}_{\mathfrak{a}}^{\text {full }}(E, \mathbb{D}):=\operatorname{Gr}_{\mathfrak{a}}^{\boldsymbol{m}(L)}(E, \mathbb{D})$, which is called the full reduction. By construction, $\operatorname{Gr}_{\mathfrak{a}}^{\text {full }}(E, \mathbb{D}) \otimes \mathcal{L}(-\mathfrak{a})$ is logarithmic.

We have functoriality as in Subsection 5.1 .2 .

Deformation. Assume $0 \notin \mathcal{K}$. We would like to regard $(E, \mathbb{D})$ as a prolongment of $(E, \mathbb{D})_{\mid \mathcal{X}-\mathcal{D}(\leq k(0))}$. For a given holomorphic function $T=T(\lambda)$ with $\operatorname{Re}(T(\lambda))>0$, we have another prolongment $\left(E^{(T)}, \mathbb{D}^{(T)}\right)$ of $(E, \mathbb{D})_{\mid \mathcal{X}-\mathcal{D}(\leq k(0))}$, which is also an unramifiedly good lattice with the set of irregular values

$$
\operatorname{Irr}\left(E^{(T)}, \mathbb{D}^{(T)}\right):=\{T(\lambda) \mathfrak{a} \mid \mathfrak{a} \in \operatorname{Irr}(\mathbb{D})\} .
$$

We refer to Subsections $4.4-4.5$ of [23] for the construction. We mention some properties:

(D1) $E^{\left(T_{1} \cdot T_{2}\right)} \simeq\left(E^{\left(T_{1}\right)}\right)^{\left(T_{2}\right)}$ if $\operatorname{Re}\left(T_{i}\right)>0$ and $\operatorname{Re}\left(T_{1} \cdot T_{2}\right)>0$.

$(\mathrm{D} 2)\left(E^{(T)}, \mathbb{D}^{(T)}\right)_{\mid \widehat{\mathcal{D}}_{I_{0}}} \simeq \bigoplus_{\mathfrak{a} \in \mathcal{I}}\left({ }^{I_{0}} \widehat{E}_{\mathfrak{a}},{ }^{I_{0}} \widehat{\mathbb{D}}_{\mathfrak{a}}+(T-1) d \mathfrak{a}\right)$, where we put $I_{0}:=$ $\{1, \ldots, k(0)\}$. Briefly, the deformation does not change the regular part. 
We give some statements on functoriality. Let $\left(E_{p}, \mathbb{D}_{p}\right)(p=1,2)$ be unramifiedly good. We have the following natural isomorphisms:

$$
\left(E_{1} \oplus E_{2}\right)^{(T)} \simeq E_{1}^{(T)} \oplus E_{2}^{(T)}, \quad\left(E_{1} \otimes E_{2}\right)^{(T)} \simeq E_{1}^{(T)} \otimes E_{2}^{(T)}, \quad\left(E^{\vee}\right)^{(T)} \simeq\left(E^{(T)}\right)^{\vee} .
$$

Here, we have assumed that $\left(E_{1}, \mathbb{D}_{1}\right) \oplus\left(E_{2}, \mathbb{D}_{2}\right)$ and $\left(E_{1}, \mathbb{D}_{1}\right) \otimes\left(E_{2}, \mathbb{D}_{2}\right)$ are unramifiedly good. Moreover, let $F:\left(E_{1}, \mathbb{D}_{1}\right) \rightarrow\left(E_{2}, \mathbb{D}_{2}\right)$ be a flat morphism. Assume $\mathcal{I}_{1} \cup \mathcal{I}_{2}$ is a good set of irregular values on the level $(\boldsymbol{m}, i(0))$. Then we have the naturally induced morphism $\left(E_{1}^{(T)}, \mathbb{D}_{1}^{(T)}\right) \rightarrow\left(E_{2}^{(T)}, \mathbb{D}_{2}^{(T)}\right)$.

\section{$\S 5.3$. Smooth divisor case}

Let $X:=\Delta^{n}$ and $D:=\left\{z_{1}=0\right\}$. Let $\mathcal{K} \subset \mathbb{C}_{\lambda}$. Let $(E, \mathbb{D})$ be an unramifiedly good lattice of a family of meromorphic $\lambda$-bundles on $(\mathcal{X}, \mathcal{D})$ with a good set of irregular values $\operatorname{Irr}(\mathbb{D})=\operatorname{Irr}(\mathbb{D}, O)$. We have the formal decomposition $(E, \mathbb{D})_{\mid \widehat{\mathcal{D}}}=$ $\bigoplus_{\mathfrak{a} \in \operatorname{Irr}(\mathbb{D})}\left(\widehat{E}_{\mathfrak{a}}, \widehat{\mathbb{D}}_{\mathfrak{a}}\right)$, where $\widehat{\mathbb{D}}_{\mathfrak{a}}-d \mathfrak{a}$ id $\widehat{E}_{\mathfrak{a}}$ are logarithmic. We set $W:=\mathcal{D} \cup \mathcal{X}^{0}$ if $0 \in \mathcal{K}$, and $W:=\mathcal{D}$ otherwise. We obtain the decomposition on $\widehat{W}$ :

$$
(E, \mathbb{D})_{\mid \widehat{W}}=\bigoplus_{\mathfrak{a} \in \operatorname{Irr}(\mathbb{D})}\left(\widehat{E}_{\mathfrak{a}}, \widehat{\mathbb{D}}_{\mathfrak{a}}\right) .
$$

Full Stokes filtration. In this case, it is also easy and convenient to consider full Stokes filtrations. (See Subsection 3.2 of [23] for the general case.) We explain this in the following. Let $\pi: \widetilde{\mathcal{X}}(W) \rightarrow \mathcal{X}$ denote the real blow up of $\mathcal{X}$ along $W$. We put $\mathfrak{Z}:=\pi^{-1}(\mathcal{D})$.

For any multi-sector $S$ in $\mathcal{X}-W$, the order $\leq_{S}$ on $\operatorname{Irr}(\mathbb{D})$ is defined as follows:

- $\mathfrak{a} \leq_{S} \mathfrak{b}$ if and only if $-\operatorname{Re}\left(\lambda^{-1} \mathfrak{a}(\lambda, \boldsymbol{z})\right) \leq_{S}-\operatorname{Re}\left(\lambda^{-1} \mathfrak{b}(\lambda, \boldsymbol{z})\right)$ for any $\boldsymbol{z} \in S$ such that $\left|z_{1}\right|$ is sufficiently small.

Let $\bar{S}$ denote the closure of $S$ in $\widetilde{\mathcal{X}}(W)$, and let $Z$ denote $\bar{S} \cap \pi^{-1}(W)$. The irregular decomposition 5.14 on $\widehat{W}$ induces the decomposition on $\widehat{Z}$ :

$$
(E, \mathbb{D})_{\mid \widehat{Z}}=\bigoplus_{\mathfrak{a} \in \operatorname{Irr}(\mathbb{D})}\left(\widehat{E}_{\mathfrak{a}}, \widehat{\mathbb{D}}_{\mathfrak{a}}\right)_{\mid \widehat{Z}}
$$

We put $\mathcal{F}_{\mathfrak{a}}^{Z}:=\bigoplus_{\mathfrak{b} \leq_{S} \mathfrak{a}} \widehat{E}_{\mathfrak{b} \mid \widehat{Z}}$, and thus we obtain the filtration $\mathcal{F}^{Z}$ indexed by $\left(\operatorname{Irr}(\mathbb{D}), \leq_{S}\right)$. By using Proposition 5.1 and Lemma 5.2 successively (or more classical results), we obtain the following.

Proposition 5.7. For any point $P \in \mathfrak{Z}$, there exists $S \in \mathcal{M S}(P, \mathcal{X}-W)$ such that:

- There exists a unique $\mathbb{D}$-flat filtration $\widetilde{\mathcal{F}}^{S}$ of $E_{\mid \bar{S}}$ indexed by $\left(\operatorname{Irr}(\mathbb{D}), \leq_{S}\right)$ such that $\widetilde{\mathcal{F}}_{\mid \widehat{Z}}^{S}=\mathcal{F}^{Z}$. 
- There exists a $\mathbb{D}$-flat splitting of $\widetilde{\mathcal{F}}^{S}$ on $\bar{S}$.

We call $\widetilde{\mathcal{F}}^{S}$ the full Stokes filtration of $(E, \mathbb{D})$. For $S^{\prime} \subset S$, the filtrations $\widetilde{\mathcal{F}}^{S^{\prime}}$ and $\widetilde{\mathcal{F}}^{S}$ satisfy the compatibility condition as in Lemma 5.2 .

The following lemma is clear from the definition of full Stokes filtrations.

Lemma 5.15. Let $S, S^{\prime} \in \mathcal{M S}(P, \mathcal{X}-W)$. Assume (i) $S^{\prime} \subset S$, (ii) $E_{\mid \bar{S}}$ has the full Stokes filtration $\widetilde{\mathcal{F}}^{S}$ as above. Then the restriction of $\widetilde{\mathcal{F}}^{S}$ to $\bar{S}^{\prime}$ is the full Stokes filtration of $E_{\mid \bar{S}^{\prime}}$.

We have functoriality of full Stokes filtrations as in the case of Stokes filtrations on the level $(\boldsymbol{m}, i(0))$.

The associated graded bundle. For any sectors $S$ and each $\mathfrak{a} \in \operatorname{Irr}(\mathbb{D})$, we obtain the bundle $\operatorname{Gr}_{\mathfrak{a}}^{\text {full }}\left(E_{\mid S}\right)$ on $S$ associated to the full Stokes filtration $\widetilde{\mathcal{F}}^{S}$. By varying $S$ and gluing $\operatorname{Gr}_{\mathfrak{a}}^{\text {full }}\left(E_{\mid \bar{S}}\right)$, we obtain the bundle $\operatorname{Gr}_{\mathfrak{a}}^{\text {full }}\left(E_{\mid \widetilde{\mathcal{V}}(W)}\right)$ on $\widetilde{\mathcal{V}}(W)$ with the induced family of flat $\lambda$-connections $\mathbb{D}_{\mathfrak{a}}$, where $\mathcal{V}$ denotes some neighbourhood of $\mathcal{D}$, and $\widetilde{\mathcal{V}}(W)$ denotes the real blow up of $\mathcal{V}$ along $W \cap \mathcal{V}$. As in Subsection 5.1.2, we can show that $\operatorname{Gr}_{\mathfrak{a}}^{\text {full }}\left(E_{\mid \widetilde{\mathcal{V}}(W)}\right)$ has descent to $\mathcal{V}$, i.e., there exists a locally free sheaf $\operatorname{Gr}_{\mathfrak{a}}^{\text {full }}(E)$ on $\mathcal{V}$ with a family of meromorphic flat $\lambda$-connections $\mathbb{D}_{\mathfrak{a}}$ such that

$$
\begin{aligned}
& \pi^{-1}\left(\mathrm{Gr}_{\mathfrak{a}}^{\text {full }}(E), \mathbb{D}_{\mathfrak{a}}\right) \simeq\left(\mathrm{Gr}_{\mathfrak{a}}^{\text {full }}\left(E_{\mid \widetilde{\mathcal{V}}(W)}\right), \mathbb{D}_{\mathfrak{a}}\right), \\
&\left(\operatorname{Gr}_{\mathfrak{a}}^{\text {full }}(E), \mathbb{D}_{\mathfrak{a}}\right)_{\mid \widehat{W} \cap \mathcal{V}} \simeq\left(\widehat{E}_{\mathfrak{a}}, \mathbb{D}_{\mathfrak{a}}\right)_{\mid \widehat{W} \cap \mathcal{V}} .
\end{aligned}
$$

By construction, $\mathbb{D}_{\mathfrak{a}}-d \mathfrak{a}$ is logarithmic for each $\mathfrak{a} \in \operatorname{Irr}(\mathbb{D})$.

As in the case of Gr with respect to Stokes filtrations on the level $(\boldsymbol{m}, i(0))$, we have the following isomorphisms:

$$
\begin{aligned}
\operatorname{Gr}_{\mathfrak{a}}^{\text {full }}\left(E^{\vee}\right) & \simeq \operatorname{Gr}_{-\mathfrak{a}}^{\text {full }}(E)^{\vee}, \\
\operatorname{Gr}_{\mathfrak{a}}^{\text {full }}\left(E_{1} \otimes E_{2}\right) & \simeq \bigoplus_{\substack{\mathfrak{a}_{i} \in \operatorname{Irr}\left(\mathbb{D}_{i}\right) \\
\mathfrak{a}_{1}+\mathfrak{a}_{2}=\mathfrak{a}}} \operatorname{Gr}_{\mathfrak{a}_{1}}^{\text {full }}\left(E_{1}\right) \otimes \operatorname{Gr}_{\mathfrak{a}_{2}}^{\text {full }}\left(E_{2}\right), \\
\operatorname{Gr}_{\mathfrak{a}}^{\text {full }}\left(E_{1} \oplus E_{2}\right) & \simeq \operatorname{Gr}_{\mathfrak{a}}^{\text {full }}\left(E_{1}\right) \oplus \operatorname{Gr}_{\mathfrak{a}}^{\text {full }}\left(E_{2}\right) .
\end{aligned}
$$

Here, we have assumed that $\left(E_{1}, \mathbb{D}_{1}\right) \otimes\left(E_{2}, \mathbb{D}_{2}\right)$ and $\left(E_{1}, \mathbb{D}_{1}\right) \oplus\left(E_{2}, \mathbb{D}_{2}\right)$ are unramifiedly good lattices.

Lemma 5.16. Let $\left(E_{p}, \mathbb{D}_{p}\right)(p=1,2)$ be unramifiedly good lattices on $(\mathcal{X}, \mathcal{D})$. Assume $\mathcal{I}_{1} \cup \mathcal{I}_{2}$ is a good set of irregular values. Let $F:\left(E_{1}, \mathbb{D}_{1}\right) \rightarrow\left(E_{2}, \mathbb{D}_{2}\right)$ be a morphism. We have the naturally induced morphism $\operatorname{Gr}_{\mathfrak{a}}^{\text {full }}(F): \operatorname{Gr}_{\mathfrak{a}}^{\text {full }}\left(E_{1}\right) \rightarrow$ $\operatorname{Gr}_{\mathfrak{a}}^{\text {full }}\left(E_{2}\right)$. 
A characterization of sections of $E$. Let $\boldsymbol{w}_{\mathfrak{a}}$ be a frame of $\operatorname{Gr}_{\mathfrak{a}}^{\text {full }}(E)$. Let $S$ be a small multi-sector, and let $E_{\mid \bar{S}}=\bigoplus E_{\mathfrak{a}, S}$ be a $\mathbb{D}$-flat splitting of the full Stokes filtration $\widetilde{\mathcal{F}}^{S}$. By the natural isomorphism $E_{\mathfrak{a}, S} \simeq \operatorname{Gr}_{\mathfrak{a}}^{\text {full }}(E)_{\mid \bar{S}}$, we take a lift $\boldsymbol{w}_{\mathfrak{a}, S}$ of $\boldsymbol{w}_{\mathfrak{a}}$. Thus, we obtain the frame $\boldsymbol{w}_{S}=\left(\boldsymbol{w}_{\mathfrak{a}, S}\right)$ of $E_{\mid \bar{S}}$. The following proposition implies a characterization of sections of $E$ by growth order with respect to the frames $\boldsymbol{w}_{S}$ for small multi-sectors $S$.

Proposition 5.8. Let $\boldsymbol{v}$ be a frame of $E$, and let $G_{S}$ be determined by $\boldsymbol{v}_{\mid S}=$ $\boldsymbol{w}_{S} \cdot G_{S}$. Then $G_{S}$ and $G_{S}^{-1}$ are bounded on $S$.

Deformation. When $|\arg (T)|$ is sufficiently small, we have a more direct local construction of the deformation $(E, \mathbb{D})^{(T)}$. We explain it in the smooth divisor case.

We take a covering $\mathcal{X}-\mathcal{D}=\bigcup_{i=1}^{N} S^{(i)}$ by sectors $S^{(i)}$ on which we have the full Stokes filtrations. Assume that $|\arg (T)|$ is so small that

- $\mathfrak{a} \leq_{S^{(i)}} \mathfrak{b} \Leftrightarrow T \mathfrak{a} \leq_{S^{(i)}} T \mathfrak{b}$ for any $\mathfrak{a}, \mathfrak{b} \in \operatorname{Irr}(\mathbb{D})$ and for any $S^{(i)}$.

We take frames $\boldsymbol{w}_{\mathfrak{a}}$ of $\operatorname{Gr}_{\mathfrak{a}}^{\text {full }}(E)$. For each $S=S^{(i)}$, we take a $\mathbb{D}$-flat splitting $E_{\mid S}=\bigoplus E_{\mathfrak{a}, S}$ of the full Stokes filtration. Let $\boldsymbol{w}_{S}=\left(\boldsymbol{w}_{\mathfrak{a}, S}\right)$ be as above. We put $\boldsymbol{w}_{\mathfrak{a}, S}^{(T)}:=\boldsymbol{w}_{\mathfrak{a}, S} \cdot \exp \left((T-1) \lambda^{-1} \mathfrak{a}\right)$ and $\boldsymbol{w}_{S}^{(T)}:=\left(\boldsymbol{w}_{\mathfrak{a}, S}^{(T)}\right)$. Let $f$ be a holomorphic section of $E_{\mid \mathcal{X}-\mathcal{D}}$. We have the corresponding decomposition $f=\sum f_{\mathfrak{a}, S}$ on each $S$. We have the expression $f_{\mathfrak{a}, S}=\sum f_{\mathfrak{a}, S, j}^{(T)} \cdot w_{\mathfrak{a}, S, j}^{(T)}$. We put $\boldsymbol{f}_{\mathfrak{a}, S}:=\left(f_{\mathfrak{a}, S, j}^{(T)}\right)$.

Lemma 5.17. $f$ gives a section of $E^{(T)}$ if and only if $\boldsymbol{f}_{\mathfrak{a}, S^{(i)}}^{(T)}$ is bounded for each $S^{(i)}$ and $\boldsymbol{w}_{S^{(i)}}$. (See Subsection 4.5 .3 of $[23$.

Prolongation of a flat morphism. Let $\left(E_{p}, \mathbb{D}_{p}\right)(p=1,2)$ be unramifiedly good lattices on $(\mathcal{X}, \mathcal{D})$. Assume $\operatorname{Irr}\left(\mathbb{D}_{1}\right) \cup \operatorname{Irr}\left(\mathbb{D}_{2}\right)$ is a good set of irregular values. Let $F:\left(E_{1}, \mathbb{D}_{1}\right)_{\mid \mathcal{X}-\mathcal{D}} \rightarrow\left(E_{2}, \mathbb{D}_{2}\right)_{\mid \mathcal{X}-\mathcal{D}}$ be a flat morphism.

Lemma 5.18. If $F$ preserves the full Stokes filtrations $\widetilde{\mathcal{F}}^{S}$ for each small sector $S$, then $F$ extends to a morphism $F: E_{1}(* \mathcal{D}) \rightarrow E_{2}(* \mathcal{D})$.

Proof. We have only to consider the case $0 \notin \mathcal{K}$ according to the Hartogs theorem. Then the claim follows from Theorem 4.3.1 of [23]. As another argument, let $\boldsymbol{w}_{S}^{(i)}$ be frames of $E_{i \mid \bar{S}}$ as in Proposition 5.8 . We can directly show that $F_{\mid S}$ is of polynomial order with respect to the frames $\boldsymbol{w}_{S}^{(i)}$.

Complement on a connection along the $\lambda$-direction. Put $X:=\Delta^{n}, D_{i}:=$ $\left\{z_{i}=0\right\}$ and $D:=\bigcup_{i=1}^{\ell} D_{i}$. Let $\mathcal{K} \subset \mathbb{C}_{\lambda}^{*}$ be a compact region. Let $(E, \mathbb{D})$ be an unramifiedly good lattice of a family of meromorphic $\lambda$-flat bundles on $(\mathcal{X}, \mathcal{D})$ 
with a good $\operatorname{set} \operatorname{Irr}(\mathbb{D})$. Assume that $E$ is equipped with a meromorphic connection along the $\lambda$-direction $\nabla_{\lambda}: E \rightarrow E \otimes \Omega_{\mathcal{K}}^{1}(* \mathcal{D})$ such that $\mathbb{D}^{f}+\nabla_{\lambda}$ is flat.

Lemma 5.19. $\nabla_{\lambda}$ naturally induces a meromorphic connection on $E^{(T)}$ along the $\lambda$-direction.

Proof. It is easy to observe that we have only to consider the case in which $D$ is smooth and $|\arg (T)|$ is sufficiently small. For $S=S^{(i)}$, let $\boldsymbol{w}_{S}=\left(\boldsymbol{w}_{\mathfrak{a}, S}\right)$ be a frame of $E_{\mid \bar{S}}$ as above. We use an argument in the proof of Lemma 5.5. Let $A_{S}=\left(A_{S, \mathfrak{a}, \mathfrak{a}^{\prime}}\right)$ be the matrix-valued holomorphic function on $S$ determined by $\nabla_{\lambda}\left(\partial_{\lambda}\right) \boldsymbol{w}_{S}=\boldsymbol{w}_{S} \cdot A_{S}$. Let $B_{\mathfrak{a}}$ be the matrix-valued holomorphic function on $\mathcal{X}$ determined by $\mathbb{D}_{\mathfrak{a}}\left(z_{1} \partial_{1}\right) \boldsymbol{w}_{\mathfrak{a}}=\boldsymbol{w}_{\mathfrak{a}} \cdot\left(z_{1} \partial_{1} \mathfrak{a}+B_{\mathfrak{a}}\right)$. Because $\left[\mathbb{D}^{f}, \nabla_{\lambda}\right]=0$, we have the following relation in the case $\mathfrak{a} \neq \mathfrak{b}$ :

$$
\lambda \cdot z_{1} \partial_{1} A_{S, \mathfrak{a}, \mathfrak{b}}+\left(z_{1} \partial_{1}(\mathfrak{a}-\mathfrak{b})\right) \cdot A_{S, \mathfrak{a}, \mathfrak{b}}+\left(A_{S, \mathfrak{a}, \mathfrak{b}} B_{\mathfrak{b}}-B_{\mathfrak{a}} A_{S, \mathfrak{a}, \mathfrak{b}}\right)=0 .
$$

Hence, $A_{S, \mathfrak{a}, \mathfrak{b}}=0$ unless $\mathfrak{a} \leq_{S} \mathfrak{b}$, and we obtain the estimate

$$
A_{S, \mathfrak{a}, \mathfrak{b}} \cdot \exp \left(\lambda^{-1}(\mathfrak{a}-\mathfrak{b})\right)=O\left(\exp \left(C\left|\lambda^{-1}\right| \cdot \log \left|z_{1}^{-1}\right|\right)\right)
$$

for some $C>0$ in the case $\mathfrak{a}<_{S} \mathfrak{b}$.

Let $A_{S}^{(T)}$ be the matrix-valued holomorphic function on $S$ determined by $\nabla\left(\partial_{\lambda}\right) \boldsymbol{w}_{S}^{(T)}=\boldsymbol{w}_{S}^{(T)} \cdot A_{S}^{(T)}$. We have $A_{S, \mathfrak{a}, \mathfrak{b}}^{(T)}=0$ unless $\mathfrak{a} \leq_{S} \mathfrak{b}$. If $\mathfrak{a}<_{S} \mathfrak{b}$, we have

$$
A_{S, \mathfrak{a}, \mathfrak{b}}^{(T)} \exp \left(\lambda^{-1} T(\mathfrak{a}-\mathfrak{b})\right)=A_{S, \mathfrak{a}, \mathfrak{b}} \exp \left(\lambda^{-1}(\mathfrak{a}-\mathfrak{b})\right)=O\left(\exp \left(C\left|\lambda^{-1}\right| \log \left|z_{1}^{-1}\right|\right)\right) .
$$

Therefore, $A_{S, \mathfrak{a}, \mathfrak{b}}^{(T)}=O\left(\exp \left(-\epsilon\left|z_{1}^{-1}\right|\right)\right)$ for some $\epsilon>0$. By a direct calculation, we obtain $A_{S, \mathfrak{a}, \mathfrak{a}}^{(T)}=A_{S, \mathfrak{a}, \mathfrak{a}}+\partial_{\lambda}\left(\lambda^{-1}(1-T) \mathfrak{a}\right)$, which is of polynomial order. Hence, the claim of the lemma follows from Lemma 5.17 .

\section{$\S 5.4$. Family of good filtered $\lambda$-flat bundles}

Pull back of a filtered bundle via a ramified covering. The notion of filtered bundle was introduced in [30] (dimension 1), and studied in 22] (arbitrary dimension). It was polished in [4, [5], [13] and [15]. Let $X$ be a complex manifold, and let $D$ be a simple normal crossing hypersurface with the irreducible decomposition $D=\bigcup_{i \in I} D_{i}$. A filtered bundle on $(X, D)$ is defined to be a sequence of locally free sheaves $\boldsymbol{E}_{*}=\left({ }_{\boldsymbol{a}} E \mid \boldsymbol{a} \in \mathbb{R}^{I}\right)$ such that (i) ${ }_{\boldsymbol{a}} E \subset{ }_{\boldsymbol{b}} E$ for $\boldsymbol{a} \leq \boldsymbol{b}$ and ${ }_{\boldsymbol{a}} E$ is the intersection of ${ }_{\boldsymbol{b}} E$ for $\boldsymbol{b}>\boldsymbol{a}$, (ii) $\boldsymbol{a}_{\boldsymbol{a}} E_{\mid X-D}={ }_{\boldsymbol{b}} E_{\mid X-D}$, (iii) ${ }_{\boldsymbol{a}} E \otimes \mathcal{O}\left(\sum n_{i} D_{i}\right)={ }_{\boldsymbol{a}-\boldsymbol{n}} E$, where $\boldsymbol{n}=\left(n_{i}\right) \in \mathbb{Z}^{I}$. In [22], we imposed some additional compatibility condition, which is slightly complicated to state. Later, Iyer and Simpson [15] introduced the notion of locally abelian condition, which is equivalent to our compatibility con- 
dition. Then Borne and Hertling-Sevenheck (4, [5, 13]) showed that such an additional compatibility condition is implied by the above three conditions.

Let us recall the pull back of a filtered bundle via a ramified covering. See 15. for a more systematic treatment. See also Subsection 2.5.3 of [23]. Put $X:=\Delta_{z}^{n}$, $D:=\bigcup_{i=1}^{\ell}\left\{z_{i}=0\right\}, \widetilde{X}:=\Delta_{w}^{n}$ and $\widetilde{D}:=\bigcup_{j=1}^{\ell}\left\{w_{j}=0\right\}$. Let $\varphi_{e}: \widetilde{X} \rightarrow X$ be a ramified covering $\varphi_{e}\left(w_{1}, \ldots, w_{n}\right)=\left(w_{1}^{e}, \ldots, w_{\ell}^{e}, w_{\ell+1}, \ldots, w_{n}\right)$. For $\boldsymbol{b} \in \mathbb{R}^{\ell}$, we put $\mathcal{S}(\boldsymbol{b}):=\left\{(\boldsymbol{a}, \boldsymbol{n}) \in \mathbb{R}^{\ell} \times \mathbb{Z}_{\geq 0}^{\ell} \mid e \cdot \boldsymbol{a}+\boldsymbol{n} \leq \boldsymbol{b}\right\}$. For a given filtered bundle $\boldsymbol{E}_{*}$ on $(X, D)$, we set

$$
{ }_{\boldsymbol{b}} \widetilde{E}=\sum_{(\boldsymbol{a}, \boldsymbol{n}) \in \mathcal{S}(\boldsymbol{b})} \boldsymbol{w}^{-\boldsymbol{n}} \cdot \varphi_{e}^{*}\left({ }_{\boldsymbol{a}} E\right) .
$$

Then it is easy to show that $\widetilde{\boldsymbol{E}}_{*}$ is also a filtered bundle. Let $\operatorname{Gal}(\widetilde{X} / X)$ denote the Galois group of the ramified covering. We can reconstruct $\boldsymbol{E}_{*}$ from $\widetilde{\boldsymbol{E}}_{*}$ with the natural $\operatorname{Gal}(\widetilde{X} / X)$-action, and hence $\boldsymbol{E}_{*}$ is called the descent of $\widetilde{\boldsymbol{E}}_{*}$. Since the construction is independent of the choice of coordinates, it can be globalized.

Family of good filtered $\lambda$-flat bundles. We use the notation of Subsection 5.2 A family of filtered $\lambda$-flat bundles on $(\mathcal{X}, \mathcal{D})$ is defined to be a filtered bundle $\boldsymbol{E}_{*}$ on $(\mathcal{X}, \mathcal{D})$ with a family of meromorphic flat $\lambda$-connections $\mathbb{D}$ on $\boldsymbol{E}=\bigcup_{a} E$.

Definition 5.5. Let $\left(\boldsymbol{E}_{*}, \mathbb{D}\right)$ be a family of filtered $\lambda$-flat bundles on $(\mathcal{X}, \mathcal{D})$.

- We say that $\left(\boldsymbol{E}_{*}, \mathbb{D}\right)$ is unramifiedly good if ${ }_{c} E$ are unramifiedly good lattices for any $c \in \mathbb{R}^{\ell}$.

- Let $P \in \mathcal{D}$. We say that $\left(\boldsymbol{E}_{*}, \mathbb{D}\right)$ is good at $P$ if there exists a ramified covering $\varphi_{e}:\left(\widetilde{\mathcal{U}}, \widetilde{\mathcal{D}}_{\mathcal{U}}\right) \rightarrow\left(\mathcal{U}, \mathcal{D}_{\mathcal{U}}\right)$ such that $\left(\widetilde{\boldsymbol{E}}_{*}, \varphi_{e}^{*} \mathbb{D}\right)$ on $\left(\widetilde{\mathcal{U}}, \widetilde{\mathcal{D}}_{\mathcal{U}}\right)$ is unramifiedly good. Here, $\mathcal{U}$ is a coordinate neighbourhood of $P, \varphi_{e}$ is a ramified covering, and $\widetilde{\boldsymbol{E}}_{*}$ is induced by $\varphi$ and $\boldsymbol{E}_{*}$ as above.

- We say that $\left(\boldsymbol{E}_{*}, \mathbb{D}\right)$ is good if it is good at any point $P \in \mathcal{D}$.

Induced filtrations. Let $\left(\boldsymbol{E}_{*}, \mathbb{D}\right)$ be a good family of filtered $\lambda$-flat bundles. Let ${ }^{i} F$ denote the induced filtration of ${ }_{c} E_{\mid \mathcal{D}_{i}}$. We set ${ }^{i} \operatorname{Gr}_{a}^{F}\left({ }_{c} E\right):={ }^{i} F_{a} /{ }^{i} F_{<a}$. It can be shown that (i) we have the well defined residue endomorphism $\operatorname{Gr}_{a}^{F} \operatorname{Res}_{i}(\mathbb{D})$ of ${ }^{i} \mathrm{Gr}_{a}^{F}\left({ }_{c} E\right)$ on $\mathcal{D}_{i}$ for each $i \in \underline{\ell}$, (ii) it preserves the induced filtrations ${ }^{j} F$ of ${ }^{i} \operatorname{Gr}_{a}^{F}\left({ }_{c} E\right)_{\mid \mathcal{D}_{i} \cap \mathcal{D}_{j}}$. (See Subsection 2.5.2 of [23]. The residues are well defined as endomorphisms of ${ }_{c} E_{\mid \mathcal{D}_{i}}$ in the non-ramified case, and as endomorphisms of ${ }^{i} \operatorname{Gr}_{a}^{F}\left({ }_{c} E\right)$ even in the ramified case. In the following, $\operatorname{Gr}_{a}^{F} \operatorname{Res}_{i}(\mathbb{D})$ are often denoted by $\operatorname{Res}_{i}(\mathbb{D})$ for simplicity.

Let $I$ be a subset of $\underline{\ell}$. We set $\mathcal{D}_{I}:=\bigcap_{i \in I} \mathcal{D}_{i}$. For $\boldsymbol{a} \in \mathbb{R}^{I}$, we put

$$
{ }^{I} F_{\boldsymbol{a}}\left({ }_{\boldsymbol{c}} E_{\mid \mathcal{D}_{I}}\right):=\bigcap_{i \in I}{ }^{i} F_{a_{i}}\left({ }_{\boldsymbol{c}} E_{\mid \mathcal{D}_{I}}\right), \quad{ }^{I} \operatorname{Gr}_{\boldsymbol{a}}^{F}\left({ }_{\boldsymbol{c}} E\right):=\frac{{ }^{I} F_{\boldsymbol{a}}\left({ }_{\boldsymbol{c}} E_{\mid \mathcal{D}_{I}}\right)}{\sum_{\boldsymbol{b} \lesseqgtr \boldsymbol{a}}{ }^{I} F_{\boldsymbol{b}}\left({ }_{c} E_{\mid \mathcal{D}_{I}}\right)} .
$$


We often consider the following sets:

$$
\mathcal{P} a r\left({ }_{\boldsymbol{c}} E, I\right):=\left\{\boldsymbol{a} \in \mathbb{R}^{I} \mid{ }^{I} \operatorname{Gr}_{\boldsymbol{a}}^{F}\left({ }_{\boldsymbol{c}} E\right) \neq 0\right\}, \quad \mathcal{P} a r\left(\boldsymbol{E}_{*}, I\right):=\bigcup_{\boldsymbol{c} \in \mathbb{R}^{\ell}} \mathcal{P} a r\left({ }_{\boldsymbol{c}} E, I\right) .
$$

We have the induced endomorphisms $\operatorname{Res}_{i}(\mathbb{D})(i \in I)$ of ${ }^{I} \operatorname{Gr}_{\boldsymbol{a}}^{F}\left({ }_{\boldsymbol{c}} E\right)$, which are mutually commutative.

KMS-structure for fixed $\lambda$. Let us consider the case in which $\mathcal{K}$ is a point $\{\lambda\}$. In this case, we prefer the symbol $\mathbb{D}^{\lambda}$ to $\mathbb{D}$. If $\lambda \neq 0$, the eigenvalues of $\operatorname{Res}_{i}\left(\mathbb{D}^{\lambda}\right)$ are constant. Hence, we have the generalized eigendecomposition ${ }^{I} \operatorname{Gr}_{\boldsymbol{a}}^{F}\left({ }_{c} E\right)=$ $\bigoplus_{\boldsymbol{\alpha}}{ }^{I} \operatorname{Gr}_{(\boldsymbol{a}, \boldsymbol{\alpha})}^{F, \mathbb{E}}\left({ }_{\boldsymbol{c}} E\right)$, where the eigenvalues of $\operatorname{Gr}^{F} \operatorname{Res}_{i}\left(\mathbb{D}^{\lambda}\right)$ on ${ }^{I} \operatorname{Gr}_{(\boldsymbol{a}, \boldsymbol{\alpha})}^{F, \mathbb{E}}\left({ }_{\boldsymbol{c}} E\right)$ are the $i$-th components of $\boldsymbol{\alpha}$. We put

$$
\begin{aligned}
\mathcal{K} \mathcal{M S}\left({ }_{\boldsymbol{c}} E, \mathbb{D}^{\lambda}, I\right) & :=\left\{(\boldsymbol{a}, \boldsymbol{\alpha}) \mid{ }^{I} \operatorname{Gr}_{(\boldsymbol{a}, \boldsymbol{\alpha})}^{F, \mathbb{E}}(\boldsymbol{c} E) \neq 0\right\} \\
\mathcal{K} \mathcal{M S}\left(\boldsymbol{E}_{*}, \mathbb{D}^{\lambda}, I\right) & :=\bigcup_{\boldsymbol{c} \in \mathbb{R}^{S}} \mathcal{K} \mathcal{M S}\left({ }_{\boldsymbol{c}} E, \mathbb{D}^{\lambda}, I\right), \\
\mathcal{S} p\left({ }_{\boldsymbol{c}} E, \mathbb{D}^{\lambda}, I\right) & :=\left\{\boldsymbol{\alpha} \in \mathbb{C}^{I} \mid \exists \boldsymbol{a} \in \mathbb{R}^{I},(\boldsymbol{a}, \boldsymbol{\alpha}) \in \mathcal{K} \mathcal{M S}\left({ }_{\boldsymbol{c}} E, \mathbb{D}^{\lambda}, I\right)\right\}, \\
\mathcal{S} p\left(\boldsymbol{E}_{*}, \mathbb{D}^{\lambda}, I\right) & :=\bigcup_{\boldsymbol{c} \in \mathbb{R}^{S}} \mathcal{S} p\left({ }_{\boldsymbol{c}} E, \mathbb{D}^{\lambda}, I\right) .
\end{aligned}
$$

Each element of $\mathcal{K} \mathcal{M S}\left(\boldsymbol{E}_{*}, \mathbb{D}^{\lambda}, I\right)$ is called a $K M S$-spectrum of $\left(\boldsymbol{E}_{*}, \mathbb{D}^{\lambda}\right)$ at $D_{I}$.

Even in the case $\lambda=0$, a similar definition makes sense if the eigenvalues of $\operatorname{Res}_{i}\left(\mathbb{D}^{\lambda}\right)$ are constant. It is satisfied when we consider wild harmonic bundles.

KMS-structure around $\lambda_{0}$. Assume that $\mathcal{K}$ is a neighbourhood of $\lambda_{0} \in \mathbb{C}$, and we regard that $\left(\boldsymbol{E}_{*}, \mathbb{D}\right)$ is given around $\left\{\lambda_{0}\right\} \times X$. In this case, we prefer the symbols ${ }^{i} F^{\left(\lambda_{0}\right)}$ to ${ }^{i} F$. Let $\mathfrak{p}(\lambda): \mathbb{R} \times \mathbb{C} \rightarrow \mathbb{R}$ and $\mathfrak{e}(\lambda): \mathbb{R} \times \mathbb{C} \rightarrow \mathbb{C}$ be given as follows:

$$
\mathfrak{p}(\lambda,(a, \alpha))=a+2 \operatorname{Re}(\lambda \cdot \bar{\alpha}), \quad \mathfrak{e}(\lambda,(a, \alpha))=\alpha-a \cdot \lambda-\bar{\alpha} \cdot \lambda^{2} .
$$

The induced map $\mathbb{R} \times \mathbb{C} \rightarrow \mathbb{R} \times \mathbb{C}$ is denoted by $\mathfrak{k}(\lambda)$.

Definition 5.6. We say that $\left(\boldsymbol{E}_{*}, \mathbb{D}\right)$ has the KMS-structure at $\lambda_{0}$ indexed by $T(i) \subset \mathbb{R} \times \mathbb{C}(i \in S)$ if:

- $\mathcal{P} a r\left(\boldsymbol{E}_{*}, i\right)$ is the image of $T(i)$ via the map $\mathfrak{p}\left(\lambda_{0}\right)$.

- For each $a \in \mathcal{P} a r\left(\boldsymbol{E}_{*}, i\right)$, we put $\mathcal{K}(a, i):=\left\{u \in T(i) \mid \mathfrak{p}\left(\lambda_{0}, u\right)=a\right\}$. Then the restrictions of $\operatorname{Res}_{i}(\mathbb{D})$ to ${ }^{i} \operatorname{Gr}_{a}^{F^{\left(\lambda_{0}\right)}}\left({ }_{c} E\right)_{\mid \mathcal{D}_{i}^{\lambda}}$ have the eigenvalues $\mathfrak{e}(\lambda, u)$ $(u \in \mathcal{K}(a, i))$ 
Assume $\left(\boldsymbol{E}_{*}, \mathbb{D}\right)$ has the KMS-structure at $\lambda_{0}$. We have the decomposition

$$
{ }^{i} \operatorname{Gr}_{a}^{F^{\left(\lambda_{0}\right)}}\left({ }_{c} E\right)=\bigoplus_{u \in \mathcal{K}(a, i)}{ }^{i} \mathcal{G}_{u}^{\left(\lambda_{0}\right)}\left({ }_{c} E\right),
$$

such that (i) it is preserved by $\operatorname{Res}_{i}(\mathbb{D})$, (ii) the restriction of $\operatorname{Res}_{i}(\mathbb{D})-\mathfrak{e}(\lambda, u)$ to ${ }^{i} \mathcal{G}_{u}^{\left(\lambda_{0}\right)}\left({ }_{c} E\right)$ is nilpotent. More generally, we have the decomposition on $\mathcal{D}_{I}$

$$
{ }^{I} \operatorname{Gr}_{\boldsymbol{a}}^{F^{\left(\lambda_{0}\right)}}\left({ }_{\boldsymbol{c}} E\right)=\bigoplus_{\boldsymbol{u} \in \prod \mathcal{K}\left(a_{i}, i\right)}{ }^{I} \mathcal{G}_{\boldsymbol{u}}^{\left(\lambda_{0}\right)}\left({ }_{\boldsymbol{c}} E\right),
$$

such that (i) it is preserved by $\operatorname{Res}_{i}(\mathbb{D})(i \in I)$, (ii) the restrictions of $\operatorname{Res}_{i}(\mathbb{D})$ $-\mathfrak{e}\left(\lambda, u_{i}\right)(i \in I)$ are nilpotent, where $u_{i}$ denotes the $i$-th component of $\boldsymbol{u}$. Note ${ }^{I} \mathcal{G}_{\boldsymbol{u}}^{\left(\lambda_{0}\right)}\left({ }_{\boldsymbol{c}} E\right)$ can be 0 .

The following lemma is standard. (See Subsection 2.8.3 of [23].)

Lemma 5.20. Let $\left(\boldsymbol{E}_{1 *}, \mathbb{D}_{1}\right)$ and $\left(\boldsymbol{E}_{2 *}, \mathbb{D}_{2}\right)$ be good filtered $\lambda$-flat bundles on $(\mathcal{X}, \mathcal{D})$ which have the KMS-structures at $\lambda_{0}$. An isomorphism $\varphi:\left(\boldsymbol{E}_{1}, \mathbb{D}_{1}\right) \simeq$ $\left(\boldsymbol{E}_{2}, \mathbb{D}_{2}\right)$ of families of meromorphic $\lambda$-flat bundles induces the isomorphism $\varphi$ : $\left(\boldsymbol{E}_{1 *}, \mathbb{D}_{1}\right) \simeq\left(\boldsymbol{E}_{2 *}, \mathbb{D}_{2}\right)$ of families of filtered $\lambda$-flat bundles.

We say that $(\boldsymbol{E}, \mathbb{D})$ has the KMS-structure at $\lambda_{0}$ if there exists a good filtered $\lambda$-flat bundle $\left(\boldsymbol{E}_{*}, \mathbb{D}\right)$ which has the KMS-structure at $\lambda_{0}$ such that $\boldsymbol{E}=\bigcup_{\boldsymbol{a}} E$. This makes sense by the above lemma.

Pick $c \in \mathbb{R}^{S}$ such that $c_{i} \notin \mathcal{P}$ ar $\left(\boldsymbol{E}_{*}, i\right)$ for each $i \in S$. Assume that $\mathcal{K}$ is a sufficiently small neighbourhood of $\lambda_{0}$. Take $\lambda_{1} \in \mathcal{K}$, and let $U\left(\lambda_{1}\right) \subset \mathcal{K}$ be a neighbourhood of $\lambda_{1}$. We set $\mathcal{X}^{\left(\lambda_{1}\right)}:=U\left(\lambda_{1}\right) \times X$. We use the symbols $\mathcal{D}_{i}^{\left(\lambda_{1}\right)}$ and $\mathcal{D}^{\left(\lambda_{1}\right)}$ in similar meanings. Let $\pi_{i, a}$ denote the projection ${ }^{i} F_{a}^{\left(\lambda_{0}\right)}\left({ }_{c} E_{\mid \mathcal{D}_{i}}\right) \rightarrow$ ${ }^{i} \operatorname{Gr}_{a}^{F^{\left(\lambda_{0}\right)}}\left({ }_{c} E\right)$ for any $a \in \mathcal{P a r}\left({ }_{c} E, i\right)$. Let $\left.\left.b \in\right] c_{i}-1, c_{i}\right]$. If $\mathfrak{p}\left(\lambda_{1}, v\right)=b$ for some $v \in \mathcal{K}(a, i)$, we put, on $\mathcal{D}_{i}^{\left(\lambda_{1}\right)}$,

$$
{ }^{i} F_{b}^{\left(\lambda_{1}\right)}:=\sum_{\substack{u \in \mathcal{K}(a, i) \\ \mathfrak{p}\left(\lambda_{1}, u\right) \leq b}} \pi_{i, a}^{-1}\left({ }^{i} \mathcal{G}_{u}^{\left(\lambda_{0}\right)}\left({ }_{c} E\right)\right) .
$$

Otherwise, let $b_{0}:=\max \left\{\mathfrak{p}\left(\lambda_{1}, v\right)<b \mid v \in \mathcal{K}(a, i)\right\}$, and set ${ }^{i} F_{b}^{\left(\lambda_{1}\right)}:={ }^{i} F_{b_{0}}^{\left(\lambda_{1}\right)}$. Thus, we obtain the filtration ${ }^{i} F^{\left(\lambda_{1}\right)}$ of ${ }_{c} E_{\mid \mathcal{D}_{i}^{\left(\lambda_{1}\right)}}$. It induces a family of filtered $\lambda$-flat bundles $\left(\boldsymbol{E}_{*}^{\left(\lambda_{1}\right)}, \mathbb{D}\right)$ on $\left(\mathcal{X}^{\left(\lambda_{1}\right)}, \mathcal{D}^{\left(\lambda_{1}\right)}\right)$. By construction, $\operatorname{Res}_{i}(\mathbb{D})-\mathfrak{e}(\lambda, u)$ are nilpotent on ${ }^{i} \operatorname{Gr}_{\mathfrak{p}\left(\lambda_{1}, u\right)}^{F^{\left(\lambda_{1}\right)}}\left({ }_{\boldsymbol{c}} E\right)$. Namely, $\left(\boldsymbol{E}_{*}^{\left(\lambda_{1}\right)}, \mathbb{D}\right)$ has the KMS-structure at $\lambda_{1}$ indexed by $T(i)$. Hence, if $(\boldsymbol{E}, \mathbb{D})$ has the KMS-structure at $\lambda_{0}$, it has the KMS-structure at any $\lambda$ sufficiently close to $\lambda_{0}$, and the index set is independent of $\lambda$. For each $\lambda \in \mathcal{K}$, we put $\boldsymbol{E}_{*}^{\lambda}:=\left(\boldsymbol{E}_{*}^{(\lambda)}\right)_{\mid \mathcal{X}^{\lambda}}$, which is a good filtered $\lambda$-flat bundle. The set $\mathcal{K} \mathcal{M S}\left(\boldsymbol{E}_{*}^{\lambda}, i\right)$ is the image of $T(i)$ via the map $\mathfrak{k}(\lambda)$. Note $\mathcal{K} \mathcal{M S}\left(\boldsymbol{E}_{*}^{0}, i\right)=T(i)$ if $0 \in \mathcal{K}$. We often identify them. 
Deformation. Let $T(\lambda)$ be a holomorphic function with $\operatorname{Re}(T(\lambda))>0$. We obtain the deformation $\left(\boldsymbol{E}_{*}^{(T)}, \mathbb{D}\right)$. If $\left(\boldsymbol{E}_{*}, \mathbb{D}\right)$ is unramified, the set of irregular values is given by

$$
\operatorname{Irr}\left(\mathbb{D}, E^{(T)}\right):=\{T \cdot \mathfrak{a} \mid \mathfrak{a} \in \operatorname{Irr}(\mathbb{D})\} .
$$

Since the regular part of the completion is unchanged, the set of KMS-spectra is unchanged.

\section{§6. Wild harmonic bundles}

\section{§6.1. Definition of wild harmonic bundle}

Local condition for Higgs fields. Let $\left(E, \bar{\partial}_{E}, \theta\right)$ be a Higgs bundle on $X-D$, where $X$ is a complex manifold, and $D$ is a normal crossing divisor of $X$. We would like to explain some conditions on the Higgs field $\theta$. First, let us consider the case $X=\Delta^{n}=\left\{\boldsymbol{z}=\left(z_{1}, \ldots, z_{n}\right)|| z_{i} \mid<1\right\}, D_{i}=\left\{z_{i}=0\right\}$ and $D=\bigcup_{i=1}^{\ell} D_{i}$. We have the expression

$$
\theta=\sum_{j=1}^{\ell} F_{j} \frac{d z_{j}}{z_{j}}+\sum_{j=\ell+1}^{n} G_{j} d z_{j} .
$$

We have the characteristic polynomials $\operatorname{det}\left(T\right.$ id $\left.-F_{j}(\boldsymbol{z})\right)=\sum A_{j, k}(\boldsymbol{z}) T^{k}$ and $\operatorname{det}\left(T\right.$ id $\left.-G_{j}(\boldsymbol{z})\right)=\sum B_{j, k}(\boldsymbol{z}) T^{k}$, where $T$ is just a formal variable. The coefficients $A_{j, k}$ and $B_{j, k}$ are holomorphic on $X-D$.

- We say that $\theta$ is tame if the following conditions are satisfied:

(T1) $A_{j, k}$ and $B_{j, k}$ are holomorphic on $X$ for any $k$.

(T2) The restriction of $A_{j, k}$ to $D_{j}$ is constant for any $j=1, \ldots, \ell$ and any $k$. In other words, the roots of $\sum A_{j, k}(\boldsymbol{z}) T^{k}$ are independent of $\boldsymbol{z} \in D_{j}$.

- We say that $\theta$ is unramifiedly good if there exists a good set of irregular values $\operatorname{Irr}(\theta) \subset M(X, D) / H(X)$ and a decomposition

$$
(E, \theta)=\bigoplus_{\mathfrak{a} \in \operatorname{Irr}(\theta)}\left(E_{\mathfrak{a}}, \theta_{\mathfrak{a}}\right)
$$

such that $\theta_{\mathfrak{a}}-d \mathfrak{a} \cdot \mathrm{id}_{E_{\mathfrak{a}}}$ are tame.

- We say that $\theta$ is $\operatorname{good}$ if $\varphi_{e}^{*}(\theta)$ is unramifiedly good for some $e \in \mathbb{Z}_{>0}$, where $\varphi_{e}$ is the covering given by $\varphi_{e}\left(z_{1}, \ldots, z_{n}\right)=\left(z_{1}^{e}, \ldots, z_{\ell}^{e}, z_{\ell+1}, \ldots, z_{n}\right)$.

Global condition for Higgs fields. Let us consider the case in which $X$ is a general complex manifold. Let $D$ be a normal crossing hypersurface of $X$, and let $(E, \theta)$ be a Higgs bundle on $X-D$. 
- We say that $\theta$ is (unramifiedly) good at $P \in D$ if it is (unramifiedly) good on some holomorphic coordinate neighbourhood of $P$.

- We say that $\theta$ is (unramifiedly) good if it is (unramifiedly) good at any point $P \in D$.

Let $Z$ be a closed analytic subset of $X$, and let $(E, \theta)$ be a Higgs bundle on $X-Z$. The Higgs field $\theta$ is called wild if there exists a regular birational map $\varphi: X^{\prime} \rightarrow X$ such that (i) $\varphi^{-1}(D)$ is normal crossing, (ii) $\varphi^{-1} \theta$ is good.

Remark 19. Even if $Z$ is a normal crossing divisor, a wild $\theta$ is not necessarily good.

Conditions for harmonic bundles. Let $X$ be a complex manifold. Let $D$ be a normal crossing hypersurface of $X$, and let $\left(E, \bar{\partial}_{E}, \theta, h\right)$ be a harmonic bundle on $X-D$.

- It is called tame if $\theta$ is tame.

- It is called an (unramifiedly) good wild harmonic bundle if $\theta$ is (unramifiedly) good.

Let $Z$ be a closed analytic subset of $X$. A harmonic bundle $\left(E, \bar{\partial}_{E}, \theta, h\right)$ on $X-Z$ is called wild if $\theta$ is wild.

Remark. We give some remarks on the condition (T2) for tameness.

1. If $\theta$ comes from a harmonic bundle $\left(E, \bar{\partial}_{E}, \theta, h\right),(\mathrm{T} 2)$ is implied by (T1). (See Lemma 8.2 of 22 .)

2. Let $\left(E, \bar{\partial}_{E}, \theta, h\right)$ be a harmonic bundle with a good set of irregular values $\operatorname{Irr}(\theta)$ and a decomposition $\left(E, \bar{\partial}_{E}, \theta\right)=\bigoplus_{\mathfrak{a} \in \operatorname{Irr}(\theta)}\left(E_{\mathfrak{a}}, \bar{\partial}_{E_{\mathfrak{a}}}, \theta_{\mathfrak{a}}\right)$ such that $\widetilde{\theta}_{\mathfrak{a}}:=$ $\theta_{\mathfrak{a}}-d \mathfrak{a} \cdot \mathrm{id}_{E_{\mathfrak{a}}}$ satisfy the condition (T1). The author does not know whether (T2) for $\widetilde{\theta}_{\mathfrak{a}}$ is automatically satisfied or not. But, if moreover $\left(E, \bar{\partial}_{E}, \theta, h\right)$ underlies a variation of polarized pure integrable twistor structure, (T2) is satisfied. Actually, the roots of the polynomials are 0. (See Lemma 7.3 below.)

\section{§6.2. Simpson's main estimate}

The first fundamental result is an estimate of Higgs field, so called Simpson's main estimate. For later use, we recall it in the case that $D$ is smooth. (See Subsections 7.2 and 7.3 of [23] for the general case.) Let $X:=\Delta^{n}$ and $D:=\left\{z_{1}=0\right\}$. Let $\left(E, \bar{\partial}_{E}, \theta, h\right)$ be an unramifiedly good wild harmonic bundle on $X-D$. We will be interested in the behaviour around $O$. Hence, by shrinking $X$, we may assume that there exists a holomorphic decomposition $(E, \theta)=\bigoplus_{(\mathfrak{a}, \alpha) \in \operatorname{Irr}(\theta) \times \mathbb{C}}\left(E_{\mathfrak{a}, \alpha}, \theta_{\mathfrak{a}, \alpha}\right)$ satisfying the following condition: 
- For the expression

$$
\theta_{\mathfrak{a}, \alpha}-\left(\alpha \cdot d z_{1} / z_{1}+d \mathfrak{a}\right) \cdot \operatorname{id}_{E_{\mathfrak{a}, \alpha}}=F_{1} \frac{d z_{1}}{z_{1}}+\sum_{j=2}^{n} G_{j} d z_{j},
$$

the coefficients of $\operatorname{det}\left(T-F_{1}\right)$ and $\operatorname{det}\left(T-G_{j}\right)$ are holomorphic on $X$, and $\operatorname{det}\left(T-F_{1}\right)_{\mid D}=T^{\operatorname{rank} E_{a, \alpha}}$.

For each $(\mathfrak{a}, \alpha)$, let $\pi_{\mathfrak{a}, \alpha}$ denote the projection onto $E_{\mathfrak{a}, \alpha}$ with respect to the decomposition. We also set $E_{\mathfrak{a}}:=\bigoplus_{\alpha \in \mathbb{C}} E_{\mathfrak{a}, \alpha}$, and let $\pi_{\mathfrak{a}}$ denote the projection onto $E_{\mathfrak{a}}$ with respect to the decomposition $E=\bigoplus_{\mathfrak{a} \in \operatorname{Irr}(\theta)} E_{\mathfrak{a}}$.

Truncation. For any $\mathfrak{a} \in \operatorname{Irr}(\theta)$, we have the expression $\mathfrak{a}=\sum_{j \leq-1} \mathfrak{a}_{j} z_{1}^{j}$. We put $\eta_{p}(\mathfrak{a}):=\sum_{j \leq p} \mathfrak{a}_{j} z_{1}^{j}$ and $\operatorname{Irr}(\theta, p):=\left\{\eta_{p}(\mathfrak{a}) \mid \mathfrak{a} \in \operatorname{Irr}(\theta)\right\}$. For each $\mathfrak{b} \in \operatorname{Irr}(\theta, p)$, let $E_{\mathfrak{b}}^{(p)}$ denote the direct sum of $E_{\mathfrak{a}}\left(\mathfrak{a} \in \operatorname{Irr}(\theta), \eta_{p}(\mathfrak{a})=\mathfrak{b}\right)$, and let $\pi_{\mathfrak{b}}^{(p)}$ denote the projection onto $E_{\mathfrak{b}}^{(p)}$ with respect to the decomposition $E=\bigoplus_{\mathfrak{b} \in \operatorname{Irr}(\theta, p)} E_{\mathfrak{b}}^{(p)}$. We have $\operatorname{Irr}(\theta,-1)=\operatorname{Irr}(\theta)$ and $E_{\mathfrak{a}}=E_{\mathfrak{a}}^{(-1)}$. We have the induced maps $\eta_{q, p}$ : $\operatorname{Irr}(\theta, p) \rightarrow \operatorname{Irr}(\theta, q)$ for $q \leq p$.

Asymptotic orthogonality. We take total orders $\leq^{\prime}$ on $\operatorname{Irr}(\theta, p)$ for $p \leq-1$ which are preserved by $\eta_{q, p}$. For each $\mathfrak{b} \in \operatorname{Irr}(\theta, p)$, we set $F_{\mathfrak{b}}^{(p)}(E):=\bigoplus_{\mathfrak{a}<\mathfrak{b}^{\mathfrak{b}}} E_{\mathfrak{a}}^{(p)}$. Let $E_{\mathfrak{b}}^{(p) \prime}$ be the orthogonal complement of $F_{<\mathfrak{b}}^{(p)}(E)$ in $F_{\mathfrak{b}}^{(p)}(E)$. We obtain an orthogonal decomposition $E=\bigoplus_{\mathfrak{a} \in \operatorname{Irr}(\theta, p)} E_{\mathfrak{a}}^{(p) '}$. Let $\pi_{\mathfrak{a}}^{(p) \prime}$ denote the orthogonal projection onto $E_{\mathfrak{a}}^{(p) \prime}$.

We take a total order $\leq^{\prime}$ on $\mathbb{C}$. Then we obtain the lexicographic order on $\operatorname{Irr}(\theta) \times \mathbb{C}$. We obtain the orthogonal decomposition $E=\bigoplus E_{\mathfrak{a}, \alpha}^{\prime}$ by the procedure as above, and let $\pi_{\mathfrak{a}, \alpha}^{\prime}$ denote the orthogonal projection onto $E_{\mathfrak{a}, \alpha}^{\prime}$.

Proposition 6.1. We have the following estimates with respect to $h$.

- $\pi_{\mathfrak{a}}^{(p)}-\pi_{\mathfrak{a}}^{(p) \prime}=O\left(\exp \left(-\epsilon\left|z_{1}^{p}\right|\right)\right)$ for some $\epsilon>0$. In particular, the decomposition $E=\bigoplus E_{\mathfrak{b}}^{(p)}$ is $O\left(\exp \left(-\epsilon\left|z_{1}^{p}\right|\right)\right)$-asymptotically orthogonal in the sense that there exists $A>0$ such that

$$
|h(u, v)| \leq A \cdot|u|_{h} \cdot|v|_{h} \cdot \exp \left(-\epsilon\left|z_{1}(Q)\right|^{p}\right)
$$

for any $Q \in X-D, u \in E_{\mathfrak{a} \mid Q}$ and $v \in E_{\mathfrak{b} \mid Q}(\mathfrak{a} \neq \mathfrak{b})$.

- $\pi_{\mathfrak{a}, \alpha}-\pi_{\mathfrak{a}, \alpha}^{\prime}=O\left(\left|z_{1}\right|^{\epsilon}\right)$ for some $\epsilon>0$. In particular, the decomposition $E=$ $\bigoplus E_{\mathfrak{a}, \alpha}$ is $O\left(\left|z_{1}\right|^{\epsilon}\right)$-asymptotically orthogonal.

Estimate of Higgs field. We set $\widetilde{\theta}:=\theta-\bigoplus_{\mathfrak{a}, \alpha}\left(d \mathfrak{a}+\alpha \cdot d z_{1} / z_{1}\right) \pi_{\mathfrak{a}, \alpha}$. Let $g_{\mathbf{p}}$ denote the Poincaré metric on $X-D$. The estimates in Subsection 7.2 of [23] imply the following. 
Proposition 6.2. $\widetilde{\theta}$ is bounded with respect to $h$ and $g_{\mathbf{p}}$.

Estimate of curvatures. As mentioned in Subsection 2.1.7, we obtain a holomorphic vector bundle $\mathcal{E}^{\lambda}=\left(E, \bar{\partial}_{E}+\lambda \theta^{\dagger}\right)$ on $X-D$. The curvature of the unitary connection associated to $\left(\mathcal{E}^{\lambda}, h\right)$ equals $-\left(1+|\lambda|^{2}\right)\left[\theta, \theta^{\dagger}\right]$.

Proposition 6.3. $\left[\theta, \theta^{\dagger}\right]$ is bounded with respect to $h$ and $g_{\mathbf{p}}$. In particular, $\left(\mathcal{E}^{\lambda}, h\right)$ is acceptable, i.e., the curvature of $\left(\mathcal{E}^{\lambda}, h\right)$ is bounded with respect to $h$ and $g_{\mathbf{p}}$.

\section{$\S 6.3$. Prolongation of unramifiedly good wild harmonic bundles}

6.3.1. Prolongment $\mathcal{P} \mathcal{E}^{\lambda}$. Let $\left(E, \bar{\partial}_{E}, \theta, h\right)$ be a good wild harmonic bundle on $X-D$, where $X$ is a complex manifold and $D$ is a normal crossing divisor. As mentioned in Subsection 2.1.7, we obtain a holomorphic vector bundle $\mathcal{E}^{\lambda}=$ $\left(E, \bar{\partial}_{E}+\lambda \theta^{\dagger}\right)$ on $X-D$ for each complex number $\lambda$. It is important to prolong it to a good filtered $\lambda$-flat bundle on $(X, D)$. For simplicity, we explain it assuming the following. (The general case can be easily reduced to this case.)

- $X=\Delta^{n}$ and $D=\bigcup_{i=1}^{\ell}\left\{z_{i}=0\right\}$.

- $\left(E, \bar{\partial}_{E}, \theta, h\right)$ is unramifiedly good wild, and the underlying Higgs bundle has a decomposition

$$
(E, \theta)=\bigoplus_{\substack{\mathfrak{a} \in \operatorname{Irr}(\theta) \\ \boldsymbol{\alpha} \in \mathbb{C}^{\ell}}}\left(E_{\mathfrak{a}, \boldsymbol{\alpha}}, \theta_{\mathfrak{a}, \boldsymbol{\alpha}}\right)
$$

such that (i) $\widetilde{\theta}_{\mathfrak{a}, \boldsymbol{\alpha}}=\theta_{\mathfrak{a}, \boldsymbol{\alpha}}-\left(d \mathfrak{a}+\sum_{j=1}^{\ell} \alpha_{j} d z_{j} / z_{j}\right) \operatorname{id}_{E_{\mathfrak{a}, \boldsymbol{\alpha}}}$ are tame, (ii) we have $\operatorname{det}\left(T \mathrm{id}-F_{j}\right)_{\mid D_{j}}=T^{\mathrm{rank} E_{\mathfrak{a}, \boldsymbol{\alpha}}}$ for the expression $\widetilde{\theta}_{\mathfrak{a}, \boldsymbol{\alpha}}=\sum_{j=1}^{\ell} F_{j} d z_{j} / z_{j}+$ $\sum_{j=\ell+1}^{n} G_{j} d z_{j}$.

For any open subset $U \subset X$ and $\boldsymbol{a} \in \mathbb{R}^{\ell}$, we set

$$
\mathcal{P}_{\boldsymbol{a}} \mathcal{E}^{\lambda}(U):=\left\{\left.f \in \mathcal{E}^{\lambda}(U \backslash D)|| f\right|_{h}=O\left(\prod_{i=1}^{\ell}\left|z_{i}\right|^{-a_{i}-\epsilon}\right) \forall \epsilon>0\right\} .
$$

Thus, by sheafification, we obtain an increasing sequence of $\mathcal{O}_{X}$-modules $\mathcal{P}_{*} \mathcal{E}^{\lambda}:=$ $\left(\mathcal{P}_{\boldsymbol{a}} \mathcal{E}^{\lambda} \mid \boldsymbol{a} \in \mathbb{R}^{\ell}\right)$. We obtain an $\mathcal{O}_{X}(* D)$-module $\mathcal{P} \mathcal{E}^{\lambda}:=\bigcup_{\boldsymbol{a}} \mathcal{P}_{\boldsymbol{a}} \mathcal{E}^{\lambda}$.

\section{Proposition 6.4.}

- (Subsection 7.4 of [23]) $\left(\mathcal{P}_{*} \mathcal{E}^{\lambda}, \mathbb{D}^{\lambda}\right)$ is an unramifiedly good filtered $\lambda$-flat bundle. The set of irregular values is given by

$$
\operatorname{Irr}\left(\mathbb{D}^{\lambda}, \mathcal{P} \mathcal{E}^{\lambda}\right)=\left\{\left(1+|\lambda|^{2}\right) \mathfrak{a} \mid \mathfrak{a} \in \operatorname{Irr}(\theta)\right\} .
$$


- (Subsection 8.2 of [23]) $\mathfrak{k}(\lambda)$ induces a bijection

$$
\mathcal{K} \mathcal{M S}\left(\mathcal{E}^{0}, i\right) \rightarrow \mathcal{K} \mathcal{M S}\left(\mathcal{E}^{\lambda}, i\right)
$$

for each $i$. We also have $\operatorname{dim}^{i} \operatorname{Gr}_{a, \alpha}^{F, \mathbb{E}}\left(\mathcal{P} \mathcal{E}^{0}\right)=\operatorname{dim}^{i} \operatorname{Gr}_{\mathfrak{k}(\lambda,(a, \alpha))}^{F, \mathbb{E}}\left(\mathcal{P} \mathcal{E}^{\lambda}\right)$.

Take an auxiliary sequence for $\operatorname{Irr}(\theta)$. Let $\operatorname{Irr}(\theta, \boldsymbol{m}(0))$ denote the image of $\operatorname{Irr}(\theta)$ via $\bar{\eta}_{\boldsymbol{m}(0)}$. If $\lambda \neq 0$, for each small sector $S$ in $\{\lambda\} \times(X-D)$, we have the Stokes filtration $\mathcal{F}^{S}$ on the level $\boldsymbol{m}(0)$, indexed by the ordered set $\left\{\left(1+|\lambda|^{2}\right) \mathfrak{a} \mid\right.$ $\mathfrak{a} \in \operatorname{Irr}(\theta, \boldsymbol{m}(0))\}$ with $\leq_{S}$. We have the following characterization of the filtration by the growth order of the norms of flat sections with respect to $h$. (See Subsection 7.4.1 of [23] for more details.)

Proposition 6.5. Assume $\lambda \neq 0$. Let $f$ be a flat section of $\mathcal{E}_{\mid S}^{\lambda}$. We have $f \in$ $\mathcal{F}_{\left(1+|\lambda|^{2}\right) \mathfrak{b}}^{S}$ for $\mathfrak{b} \in \operatorname{Irr}(\theta, \boldsymbol{m}(0))$ if and only if

$$
\left|f \cdot \exp \left(\left(\lambda^{-1}+\bar{\lambda}\right) \mathfrak{b}\right)\right|_{h}=O\left(\exp \left(C\left|z^{m(1)}\right|\right) \prod_{k(1)<j \leq \ell}\left|z_{j}\right|^{-N}\right)
$$

for some $C>0$ and $N>0$, where $k(1)$ is determined by the condition $\boldsymbol{m}(1) \in$ $\mathbb{Z}_{<0}^{k(1)} \times \mathbf{0}_{\ell-k(1)}$.

6.3.2. Prolongment $\mathcal{P}_{*}^{\left(\lambda_{0}\right)} \mathcal{E}$. It is important to consider families of $\lambda$. In the tame case, the family $\bigcup_{\lambda} \mathcal{P} \mathcal{E}^{\lambda}$ gives a regular family of meromorphic $\lambda$-flat bundles. More precisely, if we consider the sheaf of holomorphic sections of $\mathcal{E}$ of polynomial growth, then (i) it is a locally free $\mathcal{O}_{\mathcal{X}}(* \mathcal{D})$-module, (ii) the specialization at each $\{\lambda\} \times X$ is naturally isomorphic to $\mathcal{P E}^{\lambda}$. (We need some more considerations to take nice lattices.)

However, the naive family $\bigcup_{\lambda} \mathcal{P} \mathcal{E}^{\lambda}$ does not give a nice meromorphic object in the non-tame case, as suggested by the fact that the sets

$$
\operatorname{Irr}\left(\mathcal{P} \mathcal{E}^{\lambda}, \mathbb{D}^{\lambda}\right)=\left\{\left(1+|\lambda|^{2}\right) \mathfrak{a} \mid \mathfrak{a} \in \operatorname{Irr}(\theta)\right\}
$$

depend on $\lambda$ in a non-holomorphic way. We consider an auxiliary family of meromorphic $\lambda$-flat bundles $\mathcal{P}^{\left(\lambda_{0}\right)} \mathcal{E}$. We explain it in the above setting.

Let $\pi_{\mathfrak{a}, \boldsymbol{\alpha}}$ denote the projection onto $E_{\mathfrak{a}, \boldsymbol{\alpha}}$ in 6.1. We set

$$
g(\lambda):=\prod_{\mathfrak{a}, \boldsymbol{\alpha}} \exp \left(\lambda\left(\overline{\mathfrak{a}}+\sum \bar{\alpha}_{j} \log \left|z_{j}\right|^{2}\right)\right) \pi_{\mathfrak{a}, \boldsymbol{\alpha}}
$$

Let $U\left(\lambda_{0}\right)$ denote a small neighbourhood of $\lambda_{0} \in \mathbb{C}$. We set $\mathcal{X}^{\left(\lambda_{0}\right)}:=U\left(\lambda_{0}\right) \times X$ and $\mathcal{D}^{\left(\lambda_{0}\right)}:=U\left(\lambda_{0}\right) \times D$. We also set $\mathcal{X}^{\lambda}:=\{\lambda\} \times X$ and $\mathcal{D}^{\lambda}:=\{\lambda\} \times D$. Let $p_{\lambda}$ be the projection of $\mathcal{X}^{\left(\lambda_{0}\right)}-\mathcal{D}^{\left(\lambda_{0}\right)}$ onto $X-D$. We consider the hermitian metric

$$
\mathcal{P}^{\left(\lambda_{0}\right)} h:=g\left(\lambda-\lambda_{0}\right)^{*} h
$$


on $p_{\lambda}^{-1} E$ over $\mathcal{X}^{\left(\lambda_{0}\right)}-\mathcal{D}^{\left(\lambda_{0}\right)}$. Let $\boldsymbol{a} \in \mathbb{R}^{\ell}$. For any open subset $V$ of $\mathcal{X}^{\left(\lambda_{0}\right)}$, we define

$$
\mathcal{P}_{a}^{\left(\lambda_{0}\right)} \mathcal{E}(V):=\left\{\left.f \in \mathcal{E}\left(V^{*}\right)|| f\right|_{\mathcal{P}^{\left(\lambda_{0}\right)} h}=O\left(\prod_{j=1}^{\ell}\left|z_{j}\right|^{-a_{j}-\epsilon}\right), \forall \epsilon>0\right\}
$$

where $V^{*}:=V \backslash \mathcal{D}^{\left(\lambda_{0}\right)}$. Thus, by sheafification, we obtain an increasing sequence $\mathcal{P}_{*}^{\left(\lambda_{0}\right)} \mathcal{E}=\left(\mathcal{P}_{\boldsymbol{a}}^{\left(\lambda_{0}\right)} \mathcal{E} \mid \boldsymbol{a} \in \mathbb{R}^{\ell}\right)$ of $\mathcal{O}_{\mathcal{X}\left(\lambda_{0}\right)}$-modules. We put $\mathcal{P}^{\left(\lambda_{0}\right)} \mathcal{E}:=\bigcup_{\boldsymbol{a} \in \mathbb{R}^{\ell}} \mathcal{P}_{\boldsymbol{a}}^{\left(\lambda_{0}\right)} \mathcal{E}$ The restrictions to $\mathcal{X}^{\lambda}$ are denoted by $\mathcal{P}_{*}^{\left(\lambda_{0}\right)} \mathcal{E}^{\lambda}$ and $\mathcal{P}^{\left(\lambda_{0}\right)} \mathcal{E}^{\lambda}$.

\section{Proposition 6.6.}

- (Subsections 9.1 and 9.2 of [23]) $\left(\mathcal{P}_{*}^{\left(\lambda_{0}\right)} \mathcal{E}, \mathbb{D}\right)$ is an unramifiedly good family of filtered $\lambda$-flat bundles. The set of irregular values is given by

$$
\operatorname{Irr}\left(\mathcal{P}^{\left(\lambda_{0}\right)} \mathcal{E}, \mathbb{D}\right)=\left\{\left(1+\lambda \bar{\lambda}_{0}\right) \mathfrak{a} \mid \mathfrak{a} \in \operatorname{Irr}(\theta)\right\} .
$$

- (Subsection 9.2.1 of 23]) We have the deformation mentioned in Subsections 5.2 .2 and 5.4. for which $\left(\mathcal{P}^{\left(\lambda_{0}\right)} \mathcal{E}^{\lambda}, \mathbb{D}^{\lambda}\right)$ is isomorphic to $\left(\mathcal{P} \mathcal{E}^{\lambda}, \mathbb{D}^{\lambda}\right)^{T(\lambda)}$ with $T(\lambda)=\left(1+|\lambda|^{2}\right)^{-1}\left(1+\lambda \bar{\lambda}_{0}\right)$.

- (Subsection 9.2.3 of [23]) Let $U\left(\lambda_{1}\right) \subset U\left(\lambda_{0}\right)$ be small, and set $\mathcal{X}^{\left(\lambda_{1}\right)}:=$ $U\left(\lambda_{1}\right) \times X$. Then $\left(\mathcal{P}^{\left(\lambda_{1}\right)} \mathcal{E}, \mathbb{D}\right)$ on $\mathcal{X}^{\left(\lambda_{1}\right)}$ is isomorphic to the deformation $\left(\mathcal{P}^{\left(\lambda_{0}\right)} \mathcal{E}, \mathbb{D}\right)_{\mid \mathcal{X}^{\left(\lambda_{1}\right)}}^{\left(T\left(\lambda_{0}, \lambda_{1}\right)\right)}$ with $T\left(\lambda_{0}, \lambda_{1}\right)=\left(1+\lambda \bar{\lambda}_{0}\right)^{-1}\left(1+\lambda \bar{\lambda}_{1}\right)$.

We should remark that $\mathcal{P}^{\left(\lambda_{0}\right)} h \neq h$ even in the tame case, and hence $\mathcal{P}_{\boldsymbol{a}}^{\left(\lambda_{0}\right)} \mathcal{E}$ are different from ${ }_{\boldsymbol{a}} \mathcal{E}$ in 22 in the tame case. We can avoid using $\mathcal{P}_{\boldsymbol{a}}^{\left(\lambda_{0}\right)} \mathcal{E}$ by considering KMS-structure in the tame case.

By the property (D2) of the deformation (Subsection 5.2.2) and the correspondence between $\mathcal{K} \mathcal{M S}\left(\mathcal{P} \mathcal{E}^{\lambda}, i\right)$ and $\mathcal{K} \mathcal{M S}\left(\mathcal{P} \mathcal{E}^{0}, i\right)$, we can show the following.

Lemma 6.1. $\left(\mathcal{P}_{*}^{\left(\lambda_{0}\right)} \mathcal{E}, \mathbb{D}\right)$ has the $K M S$-structure at $\lambda_{0}$ indexed by $\mathcal{K} \mathcal{M S}\left(\mathcal{P} \mathcal{E}^{0}, i\right)$ $(i=1, \ldots, \ell)$.

6.3.3. Prolongment $\mathcal{Q}_{*}^{\left(\lambda_{0}\right)} \mathcal{E}$ and $\mathcal{Q} \mathcal{E}$. Applying the deformation procedure to $\left(\mathcal{P}_{*}^{\left(\lambda_{0}\right)} \mathcal{E}, \mathbb{D}\right)$ with $T=\left(1+\lambda \bar{\lambda}_{0}\right)^{-1}$, we obtain a family of good filtered $\lambda$-flat bundles $\left(\mathcal{Q}_{*}^{\left(\lambda_{0}\right)} \mathcal{E}, \mathbb{D}\right)$ on $\left(\mathcal{X}^{\left(\lambda_{0}\right)}, \mathcal{D}^{\left(\lambda_{0}\right)}\right)$. Then $\mathcal{Q}_{a}^{\left(\lambda_{0}\right)} \mathcal{E}$ is an unramifiedly good lattice of $\mathcal{Q}^{\left(\lambda_{0}\right)} \mathcal{E}$ with the good set of irregular values $\operatorname{Irr}\left(\mathcal{Q}^{\left(\lambda_{0}\right)} \mathcal{E}, \mathbb{D}\right)=\operatorname{Irr}(\theta)$. By using the property (D1) of the deformation described in Subsection 5.2.2, we obtain the following. (See Subsection 11.1 of [23] for more details.)

Lemma 6.2. The restriction $\left(\mathcal{Q}^{\left(\lambda_{0}\right)} \mathcal{E}, \mathbb{D}\right)_{\mid \mathcal{X}^{\lambda}}$ is naturally isomorphic to the deformation $\left(\mathcal{P E} \mathcal{E}^{\lambda}, \mathbb{D}^{\lambda}\right)^{\left(T_{1}(\lambda)\right)}$ with $T_{1}(\lambda)=\left(1+|\lambda|^{2}\right)^{-1}>0$. 
By the property (D1) of deformation, we have $\mathcal{Q}^{\left(\lambda_{0}\right)} \mathcal{E}_{\mid \mathcal{X}^{\left(\lambda_{1}\right)}}=\mathcal{Q}^{\left(\lambda_{1}\right)} \mathcal{E}$. Hence, we obtain the global family of meromorphic $\lambda$-flat bundles $(\mathcal{Q E}, \mathbb{D})$ on $\mathbb{C}_{\lambda} \times(X, D)$. By using the property (D2) of deformation and Lemma 6.1, we can show the following.

Lemma 6.3. $(\mathcal{Q E}, \mathbb{D})$ has the $K M S$-structure at each $\lambda_{0}$ indexed by $\mathcal{K} \mathcal{M S}\left(\mathcal{P E}^{0}, i\right)$ $(i=1, \ldots, \ell)$.

Let $S$ be a small sector in $\{\lambda\} \times(X-D)$. By Lemma 6.2, the Stokes filtrations of $\mathcal{Q} \mathcal{E}^{\lambda}$ and $\mathcal{P} \mathcal{E}^{\lambda}$ on the level $\boldsymbol{m}(0)$ are related as follows:

$$
\mathcal{F}_{\mathfrak{a}}^{S}\left(\mathcal{Q} \mathcal{E}_{\mid \bar{S}}^{\lambda}\right)=\mathcal{F}_{\left(1+|\lambda|^{2}\right) \mathfrak{a}}^{S}\left(\mathcal{P} \mathcal{E}_{\mid \bar{S}}^{\lambda}\right), \quad \mathfrak{a} \in \overline{\operatorname{Irr}}(\theta, \boldsymbol{m}(0)) .
$$

Hence, we have the characterization of the Stokes filtrations of $\mathcal{Q}$ on the level $\boldsymbol{m}(0)$ by the growth order of the norms of flat sections with respect to $h$. (See Subsection 11.1 of [23] for more details.)

Proposition 6.7. Let $f$ be a flat section of $\mathcal{E}_{\mid S}^{\lambda}$. We have $f \in \mathcal{F}_{\mathfrak{b}}^{S}\left(\mathcal{Q E}_{\mid S}^{\lambda}\right)$ for $\mathfrak{b} \in \overline{\operatorname{Irr}}(\theta, \boldsymbol{m}(0))$ if and only if

$$
\left|f \cdot \exp \left(\left(\lambda^{-1}+\bar{\lambda}\right) \mathfrak{b}\right)\right|_{h}=O\left(\exp \left(C\left|\boldsymbol{z}^{\boldsymbol{m}(1)}\right|\right) \prod_{k(1)<j \leq \ell}\left|z_{j}\right|^{-N}\right)
$$

for some $C>0$ and $N>0$, where $k(1)$ is determined by the condition $\boldsymbol{m}(1) \in$ $\mathbb{Z}_{<0}^{k(1)} \times \mathbf{0}_{\ell-k(1)}$

We obtain an unramifiedly good lattice $\left(\operatorname{Gr}_{\mathfrak{a}}^{\boldsymbol{m}(0)}(\mathcal{Q E}), \mathbb{D}_{\mathfrak{a}}\right)$ by taking $\mathrm{Gr}$ with respect to the Stokes filtration $\mathcal{F}^{S}$ on the level $\boldsymbol{m}(0)$ explained in Subsection 5.2.2.

In the case that $D$ is smooth, we have the following characterization of the full Stokes filtration $\widetilde{\mathcal{F}}^{S}$ (Subsection 11.1 of [23]).

Proposition 6.8. Let $f$ be a flat section of $\mathcal{E}_{\mid S}^{\lambda}$. We have $f \in \widetilde{\mathcal{F}}_{\mathfrak{b}}^{S}\left(\mathcal{Q E}_{\mid S}^{\lambda}\right)$ for $\mathfrak{b} \in \overline{\operatorname{Irr}}(\theta)$ if and only if

$$
\left|f \exp \left(\left(\lambda^{-1}+\bar{\lambda}\right) \mathfrak{b}\right)\right|_{h}=O\left(\left|z_{1}\right|^{-N}\right)
$$

for some $N>0$.

Remark 20. We have a characterization of full Stokes filtrations or more general Stokes filtrations on the level $\boldsymbol{m}(i)$, even in the general normal crossing case.

\section{§6.4. Reduction from wild to tame}

Let $X, D$ and $\left(E, \bar{\partial}_{E}, \theta, h\right)$ be as in Subsection 6.3. By following the same procedure for $\left(E, \partial_{E}, \theta^{\dagger}, h\right)$ on $X^{\dagger}-D^{\dagger}$, we obtain the family of meromorphic $\mu$-flat bundles $\left(\mathcal{Q} \mathcal{E}^{\dagger}, \mathbb{D}^{\dagger}\right)$ on $\mathbb{C}_{\mu} \times\left(X^{\dagger}, D^{\dagger}\right)$. 
Lemma 6.4. The correspondence $(a, \alpha) \leftrightarrow(-a, \bar{\alpha})$ induces a bijection

$$
\mathcal{K} \mathcal{M S}\left(\mathcal{P E} \mathcal{E}^{0}, i\right) \simeq \mathcal{K} \mathcal{M S}\left(\mathcal{P E} \mathcal{E}^{\dagger 0}, i\right)
$$

We also have the bijection $\operatorname{Irr}(\theta) \simeq \operatorname{Irr}\left(\theta^{\dagger}\right)$ given by $\mathfrak{a} \leftrightarrow \overline{\mathfrak{a}}$.

Proof. The claim for $\operatorname{Irr}(\theta)$ and $\operatorname{Irr}\left(\theta^{\dagger}\right)$ is clear. See Corollary 11.12 of 22 for the correspondence between $\mathcal{K} \mathcal{M S}\left(\mathcal{P} \mathcal{E}^{0}, i\right)$ and $\mathcal{K} \mathcal{M S}\left(\mathcal{P E} \mathcal{E}^{\dagger}, i\right)$.

One step reduction I. Since both the Stokes filtrations of $\left(\mathcal{Q E} \mathcal{E}^{\lambda}, \mathbb{D}^{\lambda}\right)$ and $\left(\mathcal{Q} \mathcal{E}^{\dagger \mu}, \mathbb{D}^{\dagger \mu}\right)$ are characterized by the growth order of the norms of flat sections with respect to $h$, we have the induced isomorphisms of the associated graded family of flat bundles for $\mathfrak{a} \in \operatorname{Irr}(\theta, \boldsymbol{m}(0))$ :

$$
\left(\operatorname{Gr}_{\mathfrak{a}}^{m(0)} \mathcal{Q E}, \mathbb{D}_{\mathfrak{a}}^{f}\right)_{\mid \mathbb{C}_{\lambda}^{*} \times(X-D)} \simeq\left(\operatorname{Gr}_{\overline{\mathfrak{a}}}^{m(0)} \mathcal{Q} \mathcal{E}^{\dagger}, \mathbb{D}_{\overline{\mathfrak{a}}}^{\dagger}\right)_{\mid \mathbb{C}_{\mu}^{*} \times(X-D)}
$$

Hence, they give a variation of $\mathbb{P}^{1}$-holomorphic vector bundle which is denoted by $\operatorname{Gr}_{\mathfrak{a}}^{\boldsymbol{m}(0)}\left(\mathcal{E}^{\triangle}, \mathbb{D}^{\triangle}\right)$ on $\mathbb{P}^{1} \times(X-D)$.

We can show that the pairing $\mathcal{S}:(\mathcal{E}, \mathbb{D}) \otimes \sigma^{*}\left(\mathcal{E}^{\triangle}, \mathbb{D}^{\triangle}\right) \rightarrow \mathcal{O}_{\mathcal{X}-\mathcal{D}}$ extends to

$$
\mathcal{Q E} \otimes \sigma^{*} \mathcal{Q} \mathcal{E}^{\dagger} \rightarrow \mathcal{O}_{\mathbb{C}_{\lambda} \times X}\left(*\left(\mathbb{C}_{\lambda} \times D\right)\right) .
$$

(See Subsection 11.1.3 of [23].) By functoriality of Gr with respect to Stokes structures, we obtain

$$
\operatorname{Gr}_{\mathfrak{a}}^{\boldsymbol{m}(0)}(\mathcal{Q E}, \mathbb{D}) \otimes \sigma^{*} \operatorname{Gr}_{\overline{\mathfrak{a}}}^{\boldsymbol{m}(0)}\left(\mathcal{Q} \mathcal{E}^{\dagger}, \mathbb{D}^{\dagger}\right) \rightarrow \mathcal{O}_{\mathbb{C}_{\lambda} \times X}\left(*\left(\mathbb{C}_{\lambda} \times D\right)\right)
$$

Similarly, we get $\operatorname{Gr}_{\overline{\mathfrak{a}}}^{\boldsymbol{m}(0)}\left(\mathcal{Q} \mathcal{E}^{\dagger}, \mathbb{D}^{\dagger}\right) \otimes \sigma^{*} \operatorname{Gr}_{\mathfrak{a}}^{\boldsymbol{m}(0)}(\mathcal{Q E}, \mathbb{D}) \rightarrow \mathcal{O}_{\mathbb{C}_{\mu} \times X^{\dagger}}\left(*\left(\mathbb{C}_{\mu} \times D^{\dagger}\right)\right)$. They give a morphism of variations of $\mathbb{P}^{1}$-holomorphic vector bundle on $\mathbb{P}^{1} \times(X-D)$ :

$$
\operatorname{Gr}_{\mathfrak{a}}^{\boldsymbol{m}(0)}(\mathcal{S}): \operatorname{Gr}_{\mathfrak{a}}^{\boldsymbol{m}(0)}\left(\mathcal{E}^{\triangle}, \mathbb{D}^{\triangle}\right) \otimes \sigma^{*} \operatorname{Gr}_{\mathfrak{a}}^{\boldsymbol{m}(0)}\left(\mathcal{E}^{\triangle}, \mathbb{D}^{\triangle}\right) \rightarrow \mathbb{T}(0) .
$$

One of the main results in the study of wild harmonic bundles is the following. (See Subsection 11.2 of [23] for more details.)

Proposition 6.9. If we shrink $X$ appropriately, the following holds:

- $\operatorname{Gr}_{\mathfrak{a}}^{m(0)}\left(\mathcal{E}^{\triangle}, \mathbb{D}^{\triangle}, \mathcal{S}\right)$ is a variation of pure polarized twistor structure.

- For $\mathfrak{a} \in \operatorname{Irr}(\theta, \boldsymbol{m}(0))$, let $\left(E_{\mathfrak{a}}, \bar{\partial}_{\mathfrak{a}}, h_{\mathfrak{a}}, \theta_{\mathfrak{a}}\right)$ denote the underlying harmonic bundle. By construction, the Higgs bundle $\left(E_{\mathfrak{a}}, \theta_{\mathfrak{a}}\right)$ is naturally isomorphic to

$$
\bigoplus_{\substack{\mathfrak{b} \in \operatorname{Irr}(\theta) \\ \bar{\eta}_{\boldsymbol{m}(0)}(\mathfrak{b})=\mathfrak{a}}} \bigoplus_{\boldsymbol{\alpha}}\left(E_{\mathfrak{b}, \boldsymbol{\alpha}}, \theta_{\mathfrak{b}, \boldsymbol{\alpha}}\right) .
$$

(Recall the decomposition 6.1.).) In particular, the harmonic bundle is unramifiedly good wild. The set of irregular values is $\bar{\eta}_{\boldsymbol{m}(0)}^{-1}(\mathfrak{a})$. 
- Let $\left(\mathcal{Q E}_{\mathfrak{a}}, \mathbb{D}_{\mathfrak{a}}\right)$ be the family of meromorphic $\lambda$-flat bundles on $\mathbb{C}_{\lambda} \times(X, D)$ associated to $\left(E_{\mathfrak{a}}, \bar{\partial}_{\mathfrak{a}}, h_{\mathfrak{a}}, \theta_{\mathfrak{a}}\right)$. Then we have a natural isomorphism

$$
\left(\mathcal{Q} \mathcal{E}_{\mathfrak{a}}, \mathbb{D}_{\mathfrak{a}}\right) \simeq \operatorname{Gr}_{\mathfrak{a}}^{\boldsymbol{m}(0)}(\mathcal{Q E}, \mathbb{D})
$$

- Similarly, let $\left(\mathcal{Q} \mathcal{E}_{\overline{\mathfrak{a}}}^{\dagger}, \mathbb{D}_{\frac{\mathfrak{a}}{\mathfrak{a}}}^{\dagger}\right)$ denote the associated family of meromorphic $\mu$-flat bundles on $\mathbb{C}_{\mu} \times(X, D)$. Then we have a natural isomorphism $\left(\mathcal{Q} \mathcal{E}_{\overline{\mathfrak{a}}}^{\dagger}, \mathbb{D}_{\overline{\mathfrak{a}}}^{\dagger}\right) \simeq$ $\operatorname{Gr}_{\overline{\mathfrak{a}}}^{\boldsymbol{m}(0)}\left(\mathcal{Q} \mathcal{E}^{\dagger}, \mathbb{D}^{\dagger}\right)$.

One step reduction II. Let $\overline{\operatorname{Irr}}(\theta, \boldsymbol{m}(j))$ denote the image of $\operatorname{Irr}(\theta)$ via $\bar{\eta}_{\boldsymbol{m}(j)}$. For each $\mathfrak{a} \in \overline{\operatorname{Irr}}(\theta, \boldsymbol{m}(j))$, we obtain a variation of $\mathbb{P}^{1}$-holomorphic bundle with a pairing $\operatorname{Gr}_{\mathfrak{a}}^{\boldsymbol{m}(j)}\left(\mathcal{E}^{\triangle}, \mathbb{D}^{\triangle}, \mathcal{S}\right)$, naturally isomorphic to $\operatorname{Gr}_{\mathfrak{a}}^{\boldsymbol{m}(j)} \operatorname{Gr}_{\bar{\eta}_{\boldsymbol{m}(j-1)}(\mathfrak{a})}^{\boldsymbol{m}(j-1)}\left(\mathcal{E}^{\triangle}, \mathbb{D}^{\triangle}, \mathcal{S}\right)$. We now explain how to apply Proposition 6.9 in this situation.

Let us consider the case in which $\overline{\operatorname{Irr}}(\theta, \boldsymbol{m}(j-1))$ consists of one element. We take any $\mathfrak{a} \in \operatorname{Irr}(\theta)$. Let $L(-\mathfrak{a})$ be the variation of polarized pure twistor structure as in Subsection 2.2.1. The underlying harmonic bundle is also denoted by $L(-\mathfrak{a})$. We set $\left(E^{\prime}, \bar{\partial}_{E^{\prime}}, \theta^{\prime}, h^{\prime}\right):=\left(E, \bar{\partial}_{E}, \theta, h\right) \otimes L(-\mathfrak{a})$. Note $\operatorname{Irr}\left(\theta^{\prime}\right):=\left\{\mathfrak{a}^{\prime}-\mathfrak{a} \mid \mathfrak{a}^{\prime} \in \operatorname{Irr}(\theta)\right\}$, and hence $\boldsymbol{m}(j), \boldsymbol{m}(j+1), \ldots, \boldsymbol{m}(L)$ give an auxiliary sequence for $\operatorname{Irr}\left(\theta^{\prime}\right)$. We have the natural isomorphisms of the associated variation of polarized pure twistor structure:

$$
\left(\mathcal{E}^{\triangle}, \mathbb{D}^{\triangle}, \mathcal{S}\right) \simeq\left(\mathcal{E}^{\prime \triangle}, \mathbb{D}^{\prime \triangle}, \mathcal{S}^{\prime}\right) \otimes L(\mathfrak{a})
$$

For each $\mathfrak{b} \in \operatorname{Irr}(\theta, \boldsymbol{m}(j))$, we have the natural isomorphism

$$
\operatorname{Gr}_{\mathfrak{b}}^{\boldsymbol{m}(j)}\left(\mathcal{E}^{\triangle}, \mathbb{D}^{\triangle}, \mathcal{S}\right) \simeq \operatorname{Gr}_{\mathfrak{b}-\bar{\eta}_{\boldsymbol{m}(j)}(\mathfrak{a})}^{(j)}\left(\mathcal{E}^{\prime \triangle}, \mathbb{D}^{\prime \triangle}, \mathcal{S}^{\prime}\right) \otimes L(\mathfrak{a})
$$

Hence, by shrinking $X$ appropriately, we deduce that $\operatorname{Gr}_{\mathfrak{b}}^{\boldsymbol{m}(j)}\left(\mathcal{E}^{\triangle}, \mathbb{D}^{\triangle}, \mathcal{S}\right)$ is also a variation of pure twistor structure, due to Proposition 6.9.

Full reduction. Let us consider the general case. By using the above result inductively, we see that $\operatorname{Gr}_{\mathfrak{a}}^{\boldsymbol{m}(j)}\left(\mathcal{E}^{\triangle}, \mathbb{D}^{\triangle}, \mathcal{S}\right)$ are variations of polarized pure twistor structure for any $\mathfrak{a} \in \operatorname{Irr}(\theta, \boldsymbol{m}(j))$. The underlying Higgs field is

$$
\bigoplus_{\substack{\mathfrak{b} \in \operatorname{Irr}(\theta) \\ \bar{\eta}_{\boldsymbol{m}(j)}(\mathfrak{b})=\mathfrak{a}}} \bigoplus_{\boldsymbol{\alpha}}\left(E_{\mathfrak{b}, \boldsymbol{\alpha}}, \theta_{\mathfrak{b}, \boldsymbol{\alpha}}\right) .
$$

For any $\mathfrak{a} \in \operatorname{Irr}(\theta)$, we set $\operatorname{Gr}_{\mathfrak{a}}^{\text {full }}\left(\mathcal{E}, \mathbb{D}^{\triangle}, \mathcal{S}\right):=\operatorname{Gr}_{\mathfrak{a}}^{\boldsymbol{m}(L)}\left(\mathcal{E}, \mathbb{D}^{\triangle}, \mathcal{S}\right)$; these are called the full reductions. Let $\left(E_{\mathfrak{a}}, \bar{\partial}_{\mathfrak{a}}, h_{\mathfrak{a}}\right)$ be the underlying harmonic bundles. Then $\left(E_{\mathfrak{a}}, \bar{\partial}_{\mathfrak{a}}, h_{\mathfrak{a}}\right) \otimes L(-\mathfrak{a})$ are tame. This procedure is the reduction from wild harmonic bundles to tame harmonic bundles. 


\section{$\S 6.5$. Reduction from tame to twistor nilpotent orbit}

Let $X:=\Delta^{n}, D_{i}=\left\{z_{i}=0\right\}$ and $D:=\bigcup_{i=1}^{\ell} D_{i}$. Let $\left(E, \bar{\partial}_{E}, \theta, h\right)$ be a tame harmonic bundle on $X-D$. The family of $\lambda$-flat bundles $(\mathcal{E}, \mathbb{D})$ is prolonged to a family of meromorphic $\lambda$-flat bundle $(\mathcal{Q E}, \mathbb{D})$, which has the KMS-structure at $\lambda_{0}$ indexed by $\mathcal{K} \mathcal{M S}\left(\mathcal{P} \mathcal{E}^{0}, i\right)(i=1, \ldots, \ell)$ for each $\lambda_{0} \in \mathbb{C}_{\lambda}$. For later use, we recall how to obtain the limiting mixed twistor structure. For simplicity, we assume $\mathcal{K} \mathcal{M S}\left(\mathcal{E}^{0}, i\right) \subset \mathbb{R} \times\{0\}$. See Section 11 of 22 for the general case. See also an account due to Hertling and Sevenheck in [13] for this case.

In a neighbourhood $U\left(\lambda_{0}\right)$ of $\lambda_{0}$, we set

$$
\mathcal{G}_{(\boldsymbol{a}, 0)}^{\left(\lambda_{0}\right)}(E):={ }^{\ell} \mathcal{G}_{(\boldsymbol{a}, 0)}^{\left(\lambda_{0}\right)}\left(\mathcal{Q}^{\left(\lambda_{0}\right)} \mathcal{E}\right)_{\mid U\left(\lambda_{0}\right) \times\{O\}}
$$

for $\boldsymbol{a} \in \mathcal{P} \operatorname{Pr}\left(\mathcal{P} \mathcal{E}^{0}, \underline{\ell}\right)$. (See 5.17 for the right hand side. In this simpler case, we have only to take $\mathrm{Gr}$ with respect to parabolic filtrations.) By varying $\lambda_{0} \in \mathbb{C}_{\lambda}$ and gluing them, we obtain the vector bundle $\mathcal{G}_{(\boldsymbol{a}, 0)}(E)$ on $\mathbb{C}_{\lambda}$. It is endowed with the nilpotent maps $\mathcal{N}_{i}(i=1, \ldots, \ell)$, which are the nilpotent part of the residues $\operatorname{Res}_{i}(\mathbb{D})$. By applying the same procedure to $\left(E, \partial_{E}, \theta^{\dagger}, h\right)$ on $X^{\dagger}-D^{\dagger}$, we obtain the vector bundle $\mathcal{G}_{(-\boldsymbol{a}, 0)}^{\dagger}(E)$ on $\mathbb{C}_{\mu}$ with nilpotent endomorphisms $\mathcal{N}_{i}^{\dagger}$ induced by residues $\operatorname{Res}_{i}\left(\mathbb{D}^{\dagger}\right)$. We would like to glue $\mathcal{G}_{(\boldsymbol{a}, 0)}(E)$ and $\mathcal{G}_{(-\boldsymbol{a}, 0)}^{\dagger}(E)$, to obtain a vector bundle $S_{(\boldsymbol{a}, 0)}^{\mathrm{can}}(E)$ on $\mathbb{P}^{1}$.

We have the $\mathbb{D}$-flat decomposition $\mathcal{Q}_{0} \mathcal{E}_{\mid \mathbb{C}_{\lambda}^{*} \times X}=\bigoplus_{\boldsymbol{a} \in \mathcal{P} \operatorname{ar}\left(\mathcal{P}_{0} \mathcal{E}^{0}, \underline{l}\right)} \mathcal{G}_{(\boldsymbol{a}, 0)} \mathcal{E}$ with the following property:

- Let $M_{i}$ be the family of the monodromy endomorphisms along the path $\left(z_{1}, \ldots, e^{2 \pi \sqrt{-1} \theta} z_{i}, \ldots, z_{n}\right)(0 \leq \theta \leq 1)$ with respect to $\mathbb{D}^{f}$. Then the restriction of $M_{i}$ to $\mathcal{G}_{(\boldsymbol{a}, 0)} \mathcal{E}$ has a unique eigenvalue $\exp \left(2 \pi \sqrt{-1} a_{i}\right)$.

- $\mathcal{G}_{(\boldsymbol{a}, 0)} \mathcal{E}_{\mid \mathbb{C}_{\lambda}^{*} \times O} \simeq \mathcal{G}_{(\boldsymbol{a}, 0)}(E)_{\mid \mathbb{C}_{\lambda}^{*}}$.

For $\lambda \neq 0$, let $H\left(\mathcal{E}^{\lambda}\right)$ be the space of multi-valued flat sections of $\left(\mathcal{E}^{\lambda}, \mathbb{D}^{\lambda}\right)$. We have the holomorphic vector bundle $\mathcal{H}(E)$ on $\mathbb{C}_{\lambda}^{*}$ whose fiber over $\lambda$ is $H\left(\mathcal{E}^{\lambda}\right)$. We have the decomposition

$$
\mathcal{H}(E)=\bigoplus_{\boldsymbol{a} \in \mathcal{P} \operatorname{ar}\left(\mathcal{P}_{0} \mathcal{E}^{0}, \underline{\ell}\right)} \mathcal{G}_{(\boldsymbol{a}, 0)} \mathcal{H}(E)
$$

such that (i) it is preserved by the monodromy $M_{i}$, (ii) the restriction of $M_{i}$ to $\mathcal{G}_{(\boldsymbol{a}, 0)} \mathcal{H}(E)$ has a unique eigenvalue $\exp \left(2 \pi \sqrt{-1} a_{i}\right)$.

Let $U \subset \mathbb{C}_{\lambda}^{*}$, and let $s$ be a section of $\mathcal{G}_{(\boldsymbol{a}, 0)} \mathcal{H}(E)$ on $U$. We regard $s$ as a multi-valued flat section of $\mathcal{G}_{(\boldsymbol{a}, 0)} \mathcal{E}$. It is expressed as a finite sum:

$$
s=\sum f_{\boldsymbol{m}} \cdot \prod_{i=1}^{\ell} \exp \left(a_{i} \log z_{i}\right) \cdot\left(\log z_{i}\right)^{m_{i}} .
$$


Here, $f_{\boldsymbol{m}}$ are holomorphic sections of $\mathcal{G}_{(\boldsymbol{a}, 0)} \mathcal{E}_{\mid U \times X}$. We set $\Phi_{(\boldsymbol{a}, 0)}^{\text {can }}(s)=f_{0 \mid U \times O}$, and thus we obtain an isomorphism

$$
\Phi_{(\boldsymbol{a}, 0)}^{\mathrm{can}}: \mathcal{G}_{(\boldsymbol{a}, 0)} \mathcal{H}(E) \rightarrow \mathcal{G}_{(\boldsymbol{a}, 0)} \mathcal{E}_{\mid \mathbb{C}_{\lambda}^{*} \times O}=\mathcal{G}_{(\boldsymbol{a}, 0)}(E)_{\mid \mathbb{C}_{\lambda}^{*}}
$$

Let $\boldsymbol{\delta}=(1, \ldots, 1) \in \mathbb{R}^{\ell}$. We have the $\mathbb{D}^{\dagger}$-flat decomposition $\mathcal{Q}_{<\delta} \mathcal{E}_{\mid \mathbb{C}_{\lambda}^{*} \times X}^{\dagger}=$ $\bigoplus_{\boldsymbol{a} \in \mathcal{P} \operatorname{Par}\left(\mathcal{P}_{0} \mathcal{E}^{0}, \underline{\ell}\right)} \mathcal{G}_{(-\boldsymbol{a}, 0)} \mathcal{E}^{\dagger}$ with the following properties:

- The restriction of $M_{i}^{-1}$ to $\mathcal{G}_{(-\boldsymbol{a}, 0)} \mathcal{E}^{\dagger}$ has a unique eigenvalue $\exp \left(-2 \pi \sqrt{-1} a_{i}\right)$. (Because the base space is the complex conjugate $X^{\dagger}-D^{\dagger}$, the direction of the loop is reversed.)

- $\mathcal{G}_{(-\boldsymbol{a}, 0)} \mathcal{E}_{\mid \mathbb{C}_{\mu}^{*} \times O}^{\dagger} \simeq \mathcal{G}_{(-\boldsymbol{a}, 0)}^{\dagger}(E)_{\mid \mathbb{C}_{\mu}^{*}}$.

Similarly, let $\mathcal{H}^{\dagger}(E)$ be the holomorphic vector bundle on $\mathbb{C}_{\mu}^{*}$ whose fiber over $\mu$ is the space of multi-valued flat sections of $\left(\mathcal{E}^{\dagger \mu}, \mathbb{D}^{\dagger \mu}\right)$. We have the decomposition

$$
\mathcal{H}^{\dagger}(E)=\bigoplus_{\boldsymbol{a} \in \mathcal{P} \operatorname{ar}\left(\mathcal{P}_{0} \mathcal{E}^{0}, \underline{\ell}\right)} \mathcal{G}_{(-\boldsymbol{a}, 0)} \mathcal{H}^{\dagger}(E)
$$

such that the restriction of $M_{i}^{-1}$ to $\mathcal{G}_{(-\boldsymbol{a}, 0)} \mathcal{H}^{\dagger}(E)$ has a unique eigenvalue $\exp \left(-2 \pi \sqrt{-1} a_{i}\right)$. For a section $s$ of $\mathcal{G}_{(-\boldsymbol{a}, 0)} \mathcal{H}^{\dagger}(E)_{\mid U}$, we have an expression

$$
s=\sum f_{m}^{\dagger} \prod_{i=1}^{\ell} \exp \left(-a_{i} \log \bar{z}_{i}\right) \cdot\left(\log \bar{z}_{i}\right)^{m_{i}},
$$

where $f_{\boldsymbol{m}}^{\dagger}$ are sections of $\mathcal{Q E}_{\mid U \times X}^{\dagger}$. We set $\Phi_{(\boldsymbol{a}, 0)}^{\mathrm{can} \dagger}(s)=f_{0 \mid U \times O}^{\dagger}$, and thus we obtain an isomorphism

$$
\Phi_{(\boldsymbol{a}, 0)}^{\mathrm{can} \dagger}: \mathcal{G}_{(-\boldsymbol{a}, 0)} \mathcal{H}^{\dagger}(E) \rightarrow \mathcal{G}_{(-\boldsymbol{a}, 0)} \mathcal{E}_{\mid \mathbb{C}_{\mu}^{*} \times O}^{\dagger}=\mathcal{G}_{(-\boldsymbol{a}, 0)}^{\dagger}(E)_{\mid \mathbb{C}_{\mu}^{*}} .
$$

By construction, we have the natural isomorphism $\mathcal{G}_{(\boldsymbol{a}, 0)} \mathcal{H}(E) \simeq \mathcal{G}_{(-\boldsymbol{a}, 0)} \mathcal{H}^{\dagger}(E)$ under the identification of $\mathbb{C}_{\lambda}^{*}=\mathbb{C}_{\mu}^{*}$ via $\mu=\lambda^{-1}$. Thus, we obtain the vector bundle $S_{(\boldsymbol{a}, 0)}^{\text {can }}(E)$ by gluing $\mathcal{G}_{(\boldsymbol{a}, 0)}(E)$ and $\mathcal{G}_{(-\boldsymbol{a}, 0)}^{\dagger}(E)$. Under the gluing, we have the relation

$$
\lambda^{-1} \mathcal{N}_{i}=-\mu^{-1} \mathcal{N}_{i}^{\dagger}
$$

Thus, $\mathcal{N}_{i} t_{0}^{(-1)}$ and $\mathcal{N}_{\infty} t_{\infty}^{(-1)}$ give a morphism

$$
\mathcal{N}_{i}^{\triangle}: S_{(\boldsymbol{a}, 0)}^{\mathrm{can}}(E) \rightarrow S_{(\boldsymbol{a}, 0)}^{\mathrm{can}}(E) \otimes \mathbb{T}(-1) .
$$

The tuple of these morphisms is denoted by $\boldsymbol{N}^{\triangle}$. 
The morphism $\mathcal{S}_{0}: \mathcal{E} \otimes \sigma^{*} \mathcal{E}^{\dagger} \rightarrow \mathcal{O}_{\mathcal{X}-\mathcal{D}}$ extends to $\mathcal{Q}_{0} \mathcal{E} \otimes \sigma^{*} \mathcal{Q}_{<\delta} \mathcal{E}^{\dagger} \rightarrow \mathcal{O}_{\mathcal{X}}$ Similarly, we have $\mathcal{Q}_{<\delta} \mathcal{E}^{\dagger} \otimes \sigma^{*} \mathcal{Q}_{0} \mathcal{E} \rightarrow \mathcal{O}_{\mathcal{X}^{\dagger}}$. They induce the following pairings:

$$
\begin{gathered}
\mathcal{G}_{(\boldsymbol{a}, 0)}(E) \otimes \sigma^{*} \mathcal{G}_{(-\boldsymbol{a}, 0)}^{\dagger}(E) \rightarrow \mathcal{O}_{\mathbb{C}_{\lambda}}, \\
\mathcal{G}_{(-\boldsymbol{a}, 0)}^{\dagger}(E) \otimes \sigma^{*} \mathcal{G}_{(\boldsymbol{a}, 0)}(E) \rightarrow \mathcal{O}_{\mathbb{C}_{\mu}}, \\
\mathcal{G}_{(-\boldsymbol{a}, 0)} \mathcal{H}^{\dagger}(E) \otimes \sigma^{*} \mathcal{G}_{(\boldsymbol{a}, 0)} \mathcal{H}(E) \rightarrow \mathcal{O}_{\mathbb{C}_{\lambda}^{*}} .
\end{gathered}
$$

They are preserved by the above isomorphisms. Hence, we obtain

$$
\mathcal{S}_{(\boldsymbol{a}, 0)}: S_{(\boldsymbol{a}, 0)}^{\mathrm{can}}(E) \otimes \sigma^{*} S_{(\boldsymbol{a}, 0)}^{\mathrm{can}}(E) \rightarrow \mathbb{T}(0) .
$$

Theorem 12.22 of [22] implies the following.

Proposition 6.10. The tuple $\left(S_{(\boldsymbol{a}, 0)}^{\mathrm{can}}(E), \boldsymbol{N}^{\triangle}, \mathcal{S}\right)$ is a polarized mixed twistor structure of weight 0 in $\ell$ variables.

By Theorem 4.1, a polarized mixed twistor structure induces a nilpotent orbit. This is the reduction from tame harmonic bundles to nilpotent orbits.

Remark 21. The construction explained in this subsection is the same as that in 22 , although the notation is changed. In the tame case, $\mathcal{Q E}$ is equal to the sheaf of holomorphic sections whose norms with respect to $h$ are of polynomial growth order. We also remark the uniqueness in Lemma 5.20 .

Family version. The construction can be done for families on $D_{\underline{\ell}}:=\bigcap_{i=1}^{\ell} D_{i}$. As in the construction of $\mathcal{G}_{(\boldsymbol{a}, 0)}(E)$, we obtain the vector bundle $\underline{\ell}_{(\boldsymbol{a}, 0)}(\mathcal{Q E})$ on $\mathcal{D}_{\underline{\ell}}:=$ $\mathbb{C}_{\lambda} \times D_{\underline{\ell}}$, as the gluing of $\underline{\mathcal{G}}_{(\boldsymbol{a}, 0)}^{\left(\lambda_{0}\right)}\left(\mathcal{Q}^{\left(\lambda_{0}\right)} \mathcal{E}\right)$. They are equipped with the nilpotent maps $\mathcal{N}_{i}(i=1, \ldots, \ell)$. By applying the nearby cycle functors for $\mathcal{R}$-modules along $z_{i}(i=1, \ldots, \ell)$, or by a direct consideration as in Subsection 8.8.3 of [22], we obtain the induced family of flat $\lambda$-connections $\mathbb{D}_{\boldsymbol{a}, 0}$ of $\underline{\mathcal{G}}_{(\boldsymbol{a}, 0)}(\mathcal{Q E})$ for which $\mathcal{N}_{i}$ are flat. Similarly, we obtain a family of $\mu$-flat bundles $\left(\underline{\ell}_{(-\boldsymbol{a}, 0)}\left(\mathcal{Q} \mathcal{E}^{\dagger}\right), \mathbb{D}_{-\boldsymbol{a}, 0}^{\dagger}\right)$ on $\mathbb{C}_{\mu} \times D_{\underline{\ell}}^{\dagger}$ with flat nilpotent maps $\mathcal{N}_{i}^{\dagger}$.

Let $q: X-D \rightarrow D_{\underline{\ell}}$ be the projection. We naturally obtain a holomorphic vector bundle $\widetilde{\mathcal{H}}(E)$ on $\overline{\mathbb{C}}_{\lambda}^{*} \times D_{\underline{\ell}}$, whose fiber over $(\lambda, P)$ is the space of multivalued flat sections of $\left(\mathcal{E}^{\lambda}, \mathbb{D}^{\lambda}\right)_{\mid q^{-1}(P)}$. It has the generalized eigendecomposition $\widetilde{\mathcal{H}}(E)=\bigoplus^{\ell} \mathcal{G}_{(\boldsymbol{a}, 0)} \widetilde{\mathcal{H}}(E)$ with respect to the monodromy endomorphisms around $D_{i}$ $(i=1, \ldots, \ell)$. It is naturally equipped with the family of flat connections $\mathbb{D}_{\boldsymbol{a}, 0}^{f}$.

By using the family of flat bundles $\left(\mathcal{G}_{(\boldsymbol{a}, 0)} \mathcal{E}, \mathbb{D}_{\boldsymbol{a}, 0}^{f}\right)$, we obtain flat isomorphisms

$$
\Phi_{(\boldsymbol{a}, 0)}^{\text {can }}:{\stackrel{\ell}{\mathcal{G}_{(\boldsymbol{a}, 0)}}} \tilde{\mathcal{H}}(E) \rightarrow{ }^{\ell} \mathcal{G}_{(\boldsymbol{a}, 0)}(\mathcal{Q E})_{\mid \mathbb{C}_{\lambda}^{*} \times D_{\underline{\ell}}}
$$


Similarly, we obtain flat isomorphisms

$$
\Phi_{(\boldsymbol{a}, 0)}^{\mathrm{can}}:{ }^{\ell} \mathcal{G}_{(\boldsymbol{a}, 0)} \widetilde{\mathcal{H}}(E) \rightarrow{ }^{\ell} \mathcal{G}_{(-\boldsymbol{a}, 0)}\left(\mathcal{Q \mathcal { E }}^{\dagger}\right)_{\mid \mathbb{C}_{\mu}^{*} \times D_{\underline{\underline{\ell}}}^{\dagger}}
$$

As the gluing, we obtain a variation of twistor structure $\left(\underline{\varepsilon}_{\boldsymbol{a}, 0}^{\triangle}, \mathbb{D}_{\boldsymbol{a}, 0}^{\triangle}\right)$ with a tuple $\boldsymbol{N}^{\triangle}$ of flat nilpotent morphisms

$$
\mathcal{N}_{i}^{\triangle}: \underline{\mathcal{E}}_{\boldsymbol{a}, 0}^{\triangle} \rightarrow{ }^{\ell} \mathcal{E}_{\boldsymbol{a}, 0}^{\triangle} \otimes \mathbb{T}(-1) \quad(i=1, \ldots, \ell) .
$$

We also have the induced flat symmetric pairing $\mathcal{S}: \underline{\mathcal{E}}_{\boldsymbol{a}, 0}^{\triangle} \otimes \sigma^{*} \underline{\mathcal{E}}_{\boldsymbol{a}, 0}^{\triangle} \rightarrow \mathbb{T}(0)$. By Proposition 6.10, $\left(\underline{\ell}_{\boldsymbol{a}, 0}^{\triangle}, \boldsymbol{N}^{\triangle}, \mathbb{D}_{\boldsymbol{a}, 0}^{\triangle}, \mathcal{S}_{\boldsymbol{a}, 0}\right)$ is a variation of polarized mixed twistor structure of weight 0 in $\ell$ variables. (See Subsection 2.4.1.)

\section{$\S 7$. Prolongation and reductions in the integrable case}

\section{§7.1. Preliminary estimate}

7.1.1. Statements. Let $X:=\Delta^{n}$ and $D:=\left\{z_{1}=0\right\}$. Let $\left(E, \bar{\partial}_{E}, \theta, h\right)$ be an unramifiedly good wild harmonic bundle on $X-D$. For simplicity, we assume that there exists a holomorphic decomposition

$$
(E, \theta)=\bigoplus_{\mathfrak{a} \in \operatorname{Irr}(\theta)}\left(E_{\mathfrak{a}}, \theta_{\mathfrak{a}}\right)
$$

such that each $\theta_{\mathfrak{a}}-d \mathfrak{a} \cdot \mathrm{id}_{E_{\mathfrak{a}}}$ is tame. Let $\pi_{\mathfrak{a}}$ denote the projection onto $E_{\mathfrak{a}}$ with respect to the decomposition (7.1).

Remark 22. Since $\left(E, \bar{\partial}_{E}, \theta, h\right)$ is assumed to be unramifiedly good, such a decomposition exists on a neighbourhood of each point of $D$. Because we are interested in the behaviour around $O$, we may assume such a decomposition exists globally by replacing $X$ with a small neighbourhood of $O$.

Let $\mathcal{U}$ be a holomorphic section of $\operatorname{End}(E)$ on $X-D$ such that $[\theta, \mathcal{U}]=0$. Let $\mathcal{Q}$ be a $C^{\infty}$-section of $\operatorname{End}(E)$ on $X-D$ such that $\mathcal{Q}=\mathcal{Q}^{\dagger}$. We assume the following equations hold:

$$
\begin{gathered}
\partial_{E} \mathcal{U}-[\theta, \mathcal{Q}]+\theta=0, \\
\partial_{E} \mathcal{Q}+\left[\theta, \mathcal{U}^{\dagger}\right]=0 .
\end{gathered}
$$

We set $\widetilde{\mathcal{U}}:=\mathcal{U}+\sum_{\mathfrak{a} \in \operatorname{Irr}(\theta)} \mathfrak{a} \pi_{\mathfrak{a}}$. We will prove the following proposition in Subsections 7.1 .27 .1 .6 .

Proposition 7.1. $\widetilde{\mathcal{U}}=O(1)$ and $\mathcal{Q}=O\left(\left(-\log \left|z_{1}\right|\right)^{M}\right)$ for some $M>0$ with respect to $h$. 
Remark 23. Eventually, we conclude that $\mathcal{Q}$ is bounded. (See Corollaries 7.2 and 7.3 .) See Corollary 7.1 for the boundedness of $\widetilde{\mathcal{U}}$ in the case that $D$ is normal crossing.

We set $g_{\text {irr }}(\lambda):=\exp \left(\sum \lambda \overline{\mathfrak{a}} \cdot \pi_{\mathfrak{a}}\right)$. Let $\lambda_{0} \in \mathbb{C}$, and let $U\left(\lambda_{0}\right)$ be a small neighbourhood of $\lambda_{0}$ in $\mathbb{C}$. Let $p_{\lambda}$ be the projection of $U\left(\lambda_{0}\right) \times(X-D)$ onto $X-D$. We consider the hermitian metric

$$
\mathcal{P}_{\text {irr }}^{\left(\lambda_{0}\right)} h:=g_{\text {irr }}\left(\lambda-\lambda_{0}\right)^{*} h
$$

on $p_{\lambda}^{-1} E$ over $U\left(\lambda_{0}\right) \times(X-D)$. We regard $\mathcal{U}$ and $\mathcal{Q}$ as $C^{\infty}$-sections of $\operatorname{End}\left(p_{\lambda}^{-1} E\right)$. We will prove the following proposition in Subsection 7.1.7.

Proposition 7.2. Assume $U\left(\lambda_{0}\right)$ is sufficiently small. Then $\tilde{\mathcal{U}}=O(1)$ and $\mathcal{Q}=$ $O\left(\left(-\log \left|z_{1}\right|\right)^{M}\right)$ with respect to $\mathcal{P}_{\text {irr }}^{\left(\lambda_{0}\right)} h$.

7.1.2. Preliminary. We take orthogonal decompositions $E=\bigoplus E_{\mathfrak{a}, \alpha}^{\prime}=\bigoplus E_{\mathfrak{a}}^{\prime}$ as in Subsection 6.2. For any $f \in \operatorname{End}(E)$, we have the decompositions

$$
\begin{array}{ll}
f=\sum f_{\mathfrak{a}, \mathfrak{b}}^{\prime}, & f_{\mathfrak{a}, \mathfrak{b}}^{\prime} \in \operatorname{Hom}\left(E_{\mathfrak{b}}^{\prime}, E_{\mathfrak{a}}^{\prime}\right), \\
f=\sum f_{(\mathfrak{a}, \alpha),(\mathfrak{b}, \beta)}^{\prime}, & f_{(\mathfrak{a}, \alpha),(\mathfrak{b}, \beta)}^{\prime} \in \operatorname{Hom}\left(E_{\mathfrak{b}, \beta}^{\prime}, E_{\mathfrak{a}, \alpha}^{\prime}\right) .
\end{array}
$$

We have similar decompositions for sections of $\operatorname{End}(E) \otimes \Omega^{p, q}$. The following lemma is easy to show by using Proposition 6.1.

Lemma 7.1. Let $f$ be a $C^{\infty}$-section of $\operatorname{End}(E)$ such that $f$ commutes with $\theta$.

- If $\mathfrak{a} \neq \mathfrak{b}$, we have $\left|f_{\mathfrak{a}, \mathfrak{b}}^{\prime}\right|_{h}=O\left(\exp \left(-\epsilon\left|z_{1}\right|^{\operatorname{ord}(\mathfrak{a}-\mathfrak{b})}\right)\right) \cdot|f|_{h}$ for some $\epsilon>0$.

- If $\alpha \neq \beta$, we have $\left|f_{(\mathfrak{a}, \alpha),(\mathfrak{a}, \beta)}^{\prime}\right|_{h}=O\left(\left|z_{1}\right|^{\epsilon}\right) \cdot|f|_{h}$ for some $\epsilon>0$.

7.1.3. Step 1. Let $\theta_{1}$ denote the $d z_{1}$-component of $\theta$.

Lemma 7.2. We have the following estimate with respect to $h$ :

$$
\left[\theta_{1}^{\dagger}, \mathcal{U}\right]=O\left(\frac{d \bar{z}_{1}}{\left|z_{1}\right|\left(-\log \left|z_{1}\right|\right)}\right) \cdot|\mathcal{U}|_{h} .
$$

Proof. In the following, $\epsilon_{i}$ denote some positive constants. We have the decomposition

$$
\left[\theta_{1}^{\dagger}, \mathcal{U}\right]=\sum_{\mathfrak{a}, \mathfrak{b}, \mathfrak{c}}\left(\theta_{1, \mathfrak{a}, \mathfrak{b}}^{\dagger \prime} \circ \mathcal{U}_{\mathfrak{b}, \mathfrak{c}}^{\prime}-\mathcal{U}_{\mathfrak{a}, \mathfrak{b}}^{\prime} \circ \theta_{1, \mathfrak{b}, \mathfrak{c}}^{\dagger \prime}\right) .
$$

By the estimates in Subsection 7.2 of [23] (see Subsection 6.2), we have the following estimates for $\mathfrak{a} \neq \mathfrak{b}$ :

$$
\theta_{1, \mathfrak{a}, \mathfrak{b}}^{\dagger \prime}=O\left(\exp \left(-\epsilon_{1}\left|z_{1}\right|^{\operatorname{ord}(\mathfrak{a}-\mathfrak{b})}\right) \cdot d \bar{z}_{1}\right) .
$$


Because $\mathcal{U}$ and $\theta$ are commutative, we have the following estimate for $\mathfrak{a} \neq \mathfrak{b}$ due to Lemma 7.1

$$
\mathcal{U}_{\mathfrak{a}, \mathfrak{b}}^{\prime}=O\left(\exp \left(-\epsilon_{2}\left|z_{1}\right|^{-1}\right)\right) \cdot|\mathcal{U}|_{h}
$$

Hence, we have the following estimate with respect to $h$ :

$$
\left[\theta_{1}^{\dagger}, \mathcal{U}\right]=\sum_{\mathfrak{a}}\left[\theta_{1, \mathfrak{a}, \mathfrak{a}}^{\dagger \prime}, \mathcal{U}_{\mathfrak{a}, \mathfrak{a}}^{\prime}\right]+O\left(\exp \left(-\epsilon_{3}\left|z_{1}\right|^{-1}\right) \cdot d \bar{z}_{1}\right) \cdot|\mathcal{U}|_{h}
$$

Similarly, we have the following estimates for $\alpha \neq \beta$, by Theorem 7.2.4 of [23] and Lemma 7.1 .

$$
\theta_{1,(\mathfrak{a}, \alpha),(\mathfrak{a}, \beta)}^{\dagger \prime}=O\left(\left|z_{1}\right|^{\epsilon_{4}}\right) \cdot \frac{d \bar{z}_{1}}{\bar{z}_{1}}, \quad \mathcal{U}_{(\mathfrak{a}, \alpha),(\mathfrak{a}, \beta)}^{\prime}=O\left(\left|z_{1}\right|^{\epsilon_{4}}\right) .
$$

By Proposition 6.2. $\theta_{(\mathfrak{a}, \alpha),(\mathfrak{a}, \alpha)}^{\dagger \prime}-\left(d \mathfrak{a}+\alpha \cdot d z_{1} / z_{1}\right) \cdot \pi_{\mathfrak{a}, \alpha}^{\prime}$ is bounded with respect to $h$ and Poincaré metric on $X-D$. Hence, we obtain

$$
\begin{aligned}
{\left[\theta_{1}^{\dagger}, \mathcal{U}\right] } & =\sum_{\mathfrak{a}, \alpha}\left[\theta_{1,(\mathfrak{a}, \alpha),(\mathfrak{a}, \alpha)}^{\dagger \prime}, \mathcal{U}_{(\mathfrak{a}, \alpha),(\mathfrak{a}, \alpha)}^{\prime}\right]+O\left(\left|z_{1}\right|^{\epsilon_{5}}\right) \frac{d \bar{z}_{1}}{\bar{z}_{1}}|\mathcal{U}|_{h} \\
& =O\left(\frac{d \bar{z}_{1}}{\left|z_{1}\right|\left(-\log \left|z_{1}\right|\right)}\right) \cdot|\mathcal{U}|_{h}
\end{aligned}
$$

7.1.4. Step 2. Let $\bar{\partial}_{1}$ denote the $d \bar{z}_{1}$-components of $\bar{\partial}_{E}$ and $\bar{\partial}$. Similarly, let $\partial_{1}$ denote the $d z_{1}$-component of $\partial_{E}$ and $\partial$. Then

$$
\begin{aligned}
\bar{\partial}_{1}|\mathcal{U}|_{h}^{2} & =\left(\mathcal{U}, \partial_{1} \mathcal{U}\right)_{h}=\left(\mathcal{U},\left[\theta_{1}, \mathcal{Q}\right]-\theta_{1}\right)_{h}=-\operatorname{tr}\left(\mathcal{U}\left[\theta_{1}^{\dagger}, \mathcal{Q}\right]\right)-\operatorname{tr}\left(\mathcal{U} \theta_{1}^{\dagger}\right) \\
& =-\operatorname{tr}\left(\left[\mathcal{U}, \theta_{1}^{\dagger}\right] \mathcal{Q}\right)-\operatorname{tr}\left(\mathcal{U} \theta_{1}^{\dagger}\right)
\end{aligned}
$$

Hence, we obtain

$$
\bar{\partial}_{1}|\mathcal{U}|_{h}^{2}=O\left(\frac{d \bar{z}_{1}}{\left|z_{1}\right|\left(-\log \left|z_{1}\right|\right)}\right) \cdot|\mathcal{U}|_{h} \cdot|\mathcal{Q}|_{h}+O\left(\frac{d \bar{z}_{1}}{\left|z_{1}\right|^{N}}\right) \cdot|\mathcal{U}|_{h}
$$

We also have

$$
\bar{\partial}_{1}|\mathcal{Q}|_{h}^{2}=-\left(\mathcal{Q},\left[\theta_{1}, \mathcal{U}^{\dagger}\right]\right)_{h}+\left(\left[\theta_{1}^{\dagger}, \mathcal{U}\right], \mathcal{Q}\right)_{h}=O\left(\frac{d \bar{z}_{1}}{\left|z_{1}\right|\left(-\log \left|z_{1}\right|\right)}\right) \cdot|\mathcal{U}|_{h} \cdot|\mathcal{Q}|_{h}
$$

Therefore,

$$
\bar{\partial}_{1}\left(|\mathcal{U}|_{h}^{2}+|\mathcal{Q}|_{h}^{2}\right)=O\left(\frac{d \bar{z}_{1}}{\left|z_{1}\right|\left(-\log \left|z_{1}\right|\right)}\right) \cdot|\mathcal{U}|_{h} \cdot|\mathcal{Q}|_{h}+O\left(\frac{d \bar{z}_{1}}{\left|z_{1}\right|^{N}}\right) \cdot|\mathcal{U}|_{h}
$$

We set $r:=\left|z_{1}\right|$ and $F:=\left(|\mathcal{U}|_{h}^{2}+|\mathcal{Q}|_{h}^{2}+1\right)^{1 / 2}$. We use the polar coordinates $\left(r, \arg \left(z_{1}\right), z_{2}, \ldots, z_{n}\right)$. We consider the estimate on a simply connected 
region $Z\left(\vartheta_{0}, \vartheta_{1}\right):=\left\{\vartheta_{0}<\arg \left(z_{1}\right)<\vartheta_{1}\right\}$ for some fixed $\vartheta_{0}<\vartheta_{1}$. We obtain the following estimate from 7.5 :

$$
\frac{\partial}{\partial r} F^{2}=G_{1} \cdot F^{2}+G_{2} \cdot F, \quad G_{1}=O\left(\frac{1}{r(-\log r)}\right), \quad G_{2}=O\left(\frac{1}{r^{N}}\right) .
$$

We take a solution $H \neq 0$ of the differential equation

$$
\frac{\partial}{\partial r} H=-G_{1} \cdot H
$$

Note $\log \left|H_{1}\right|=O(\log (-\log r))$. Since $Z\left(\vartheta_{0}, \vartheta_{1}\right)$ is simply connected, we can take $H^{1 / 2}$. Then we have

$$
\frac{\partial}{\partial r}\left(H \cdot F^{2}\right)=G_{2} \cdot H \cdot F=\left(G_{2} \cdot H^{1 / 2}\right) \cdot\left(H^{1 / 2} \cdot F\right) .
$$

Because $G_{2} \cdot H^{1 / 2}=O\left(r^{-M_{1}}\right)$, we obtain $H \cdot F^{2}=O\left(r^{-M_{2}}\right)$, and hence $F=$ $O\left(r^{-M_{3}}\right)$. Thus, we obtain the following estimates on $Z\left(\vartheta_{0}, \vartheta_{1}\right)$ for some $M_{4}>0$ :

$$
|\mathcal{U}|_{h}=O\left(r^{-M_{4}}\right), \quad|\mathcal{Q}|_{h}=O\left(r^{-M_{4}}\right) .
$$

By varying $\vartheta_{0}$ and $\vartheta_{1}$, we obtain the estimates 7.6 on $X-D$. In particular, we obtain the following estimate on $X-D$ for $\mathfrak{a} \neq \mathfrak{b}$ :

$$
\mathcal{U}_{\mathfrak{a}, \mathfrak{b}}^{\prime}=O\left(\exp \left(-\epsilon\left|z_{1}\right|^{\operatorname{ord}(\mathfrak{a}-\mathfrak{b})}\right)\right) .
$$

7.1.5. Step 3. We have $\left[\theta_{1}, \mathcal{U}^{\dagger}\right]=\left[\theta_{1}, \widetilde{\mathcal{U}}^{\dagger}\right]+O\left(\exp \left(-\epsilon\left|z_{1}\right|^{-1}\right) \cdot d z_{1}\right)$ with respect to $h$. By an argument in the proof of Lemma 7.2 , we obtain the following estimate with respect to $h$ :

$$
\left[\theta_{1}, \mathcal{U}^{\dagger}\right]=O\left(\frac{d z_{1}}{\left|z_{1}\right|\left(-\log \left|z_{1}\right|\right)}\right) \cdot|\widetilde{\mathcal{U}}|_{h}+O\left(\exp \left(-\epsilon\left|z_{1}\right|^{-1}\right) \cdot d z_{1}\right) .
$$

According to an estimate in Subsection 7.5.2 of [23], we have

$$
\partial_{1} \mathcal{U}=\partial_{1} \widetilde{\mathcal{U}}-\sum_{\mathfrak{a} \in \operatorname{Irr}(\theta)} \partial_{1} \mathfrak{a} \cdot \pi_{\mathfrak{a}}+O\left(\exp \left(-\epsilon\left|z_{1}\right|^{-1}\right) \cdot d z_{1}\right) .
$$

We set $\widetilde{\theta}:=\theta-\sum_{\mathfrak{a} \in \operatorname{Irr}(\theta)} d \mathfrak{a} \cdot \pi_{\mathfrak{a}}$. We obtain the following estimates with respect to $h$ :

$$
\begin{aligned}
\partial_{1} \widetilde{\mathcal{U}}-\left[\theta_{1}, \mathcal{Q}\right]+\widetilde{\theta}_{1} & =O\left(\exp \left(-\epsilon\left|z_{1}\right|^{-1}\right)\right), \\
\partial_{1} \mathcal{Q}+\left[\theta_{1}, \widetilde{\mathcal{U}}^{\dagger}\right] & =O\left(\exp \left(-\epsilon\left|z_{1}\right|^{-1}\right)\right) .
\end{aligned}
$$

We set $\widetilde{F}:=\left(|\widetilde{\mathcal{U}}|_{h}^{2}+|\mathcal{Q}|_{h}^{2}+1\right)^{1 / 2}$. As in Step 2, we consider the estimates on $Z\left(\vartheta_{0}, \vartheta_{1}\right)$. By using an argument in Subsection 7.1.4 we obtain

$$
\frac{\partial}{\partial r} \widetilde{F}^{2}=\widetilde{G}_{1} \cdot \widetilde{F}^{2}+\widetilde{G}_{2} \cdot \widetilde{F}, \quad \widetilde{G}_{1}=O\left(\frac{1}{r(-\log r)}\right), \quad \widetilde{G}_{2}=O\left(\frac{1}{r}\right) .
$$


We take a solution $\widetilde{H}_{1} \neq 0$ of the differential equation

$$
\frac{\partial}{\partial r} \widetilde{H}_{1}=-\widetilde{G}_{1} \cdot \widetilde{H}_{1}
$$

Note $\log \left|\widetilde{H}_{1}\right|=O(\log (-\log r))$. By choosing $\widetilde{H}_{1}^{1 / 2}$, we obtain

$$
\frac{\partial}{\partial r}\left(\widetilde{H}_{1} \cdot \widetilde{F}^{2}\right)=\left(\widetilde{G}_{2} \cdot \widetilde{H}_{1}^{1 / 2}\right) \cdot\left(\widetilde{H}_{1}^{1 / 2} \cdot \widetilde{F}\right) .
$$

Because $\widetilde{G}_{2} \cdot \widetilde{H}_{1}^{1 / 2}=O\left(r^{-1}(-\log r)^{M_{5}}\right)$ for some $M_{5}>0$, we obtain $\widetilde{H}_{1} \cdot \widetilde{F}^{2}=$ $O\left((-\log r)^{M_{6}}\right)$ for some $M_{6}>0$, and thus $\widetilde{F}=O\left((-\log r)^{M_{7}}\right)$ for some $M_{7}>0$. Therefore, we obtain the following estimates with respect to $h$ :

$$
\widetilde{\mathcal{U}}=O\left((-\log r)^{M_{7}}\right), \quad \mathcal{Q}=O\left((-\log r)^{M_{7}}\right) .
$$

7.1.6. Step 4. By 7.10, $\widetilde{\mathcal{U}}$ is a holomorphic section of $\mathcal{P}_{0} \operatorname{End}(E)$. Because $[\theta, \widetilde{\mathcal{U}}]=0$, we obtain the boundedness of $|\widetilde{\mathcal{U}}|_{h}$ by an estimate in Subsection 7.7 of [23]. Thus, the proof of Proposition 7.1 is finished.

Remark 24. From 7.7 and 7.9 , we also have the following estimate:

$$
\partial_{1} \mathcal{Q}=O\left(\frac{d z_{1}}{\left|z_{1}\right|\left(-\log \left|z_{1}\right|\right)}\right)
$$

Hence, we actually obtain $\mathcal{Q}=O\left(\log \left(-\log \left|z_{1}\right|\right)\right)$. However, we will obtain the boundedness later.

7.1.7. Proof of Proposition 7.2, For an endomorphism $f$ of $E$, we have

$$
|f|_{\mathcal{P}_{\text {irr }}^{\left(\lambda_{0}\right)} h}=\left|g_{\text {irr }}\left(\lambda-\lambda_{0}\right) \circ f \circ g_{\text {irr }}\left(\lambda-\lambda_{0}\right)^{-1}\right|_{h} .
$$

Hence, the claim for $\tilde{\mathcal{U}}$ is clear from $\left[\tilde{\mathcal{U}}, g_{\text {irr }}\left(\lambda-\lambda_{0}\right)\right]=0$. We have the decomposition $\mathcal{P}_{0} \mathcal{E}^{0}=\bigoplus \mathcal{P}_{0} \mathcal{E}_{\mathfrak{a}}^{0}$ extending $E=\bigoplus E_{\mathfrak{a}}$. Let $\boldsymbol{v}=\left(\boldsymbol{v}_{\mathfrak{a}}\right)$ be a holomorphic frame of $\mathcal{P}_{0} \mathcal{E}^{0}$ compatible with the decomposition. Let $C$ be the matrix-valued function determined by $\partial_{1} \boldsymbol{v}=\boldsymbol{v} \cdot C \cdot d z_{1}$. We have the decomposition $C=\left(C_{\mathfrak{a}, \mathfrak{b}}\right)$ into blocks, corresponding to the decomposition $\boldsymbol{v}=\left(\boldsymbol{v}_{\mathfrak{a}}\right)$. According to an estimate in Subsection 7.5.2 of [23], there exists $\epsilon_{1}>0$ such that, for $\mathfrak{a} \neq \mathfrak{b}$,

$$
C_{\mathfrak{a}, \mathfrak{b}}=O\left(\exp \left(-\epsilon_{1}\left|z_{1}\right|^{\operatorname{ord}(\mathfrak{a}-\mathfrak{b})}\right)\right) .
$$

Let $A$ be the matrix-valued function determined by $\mathcal{U} \boldsymbol{v}=\boldsymbol{v} \cdot A$. Note $A$ is blockdiagonal, i.e., $A=\bigoplus A_{\mathfrak{a}, \mathfrak{a}}$. We have $\left(\partial_{1} \mathcal{U}\right) \boldsymbol{v}=\boldsymbol{v} \cdot\left(\partial_{1} A+[C, A] d z_{1}\right)$. We set $B d z_{1}:=\partial_{1} A+[C, A] d z_{1}=\left(B_{\mathfrak{a}, \mathfrak{b}} d z_{1}\right)$. Then there exists $\epsilon_{2}>0$ such that, for $\mathfrak{a} \neq \mathfrak{b}$,

$$
B_{\mathfrak{a}, \mathfrak{b}}=C_{\mathfrak{a}, \mathfrak{b}} A_{\mathfrak{b}, \mathfrak{b}}-A_{\mathfrak{a}, \mathfrak{a}} C_{\mathfrak{a}, \mathfrak{b}}=O\left(\exp \left(-\epsilon_{2}\left|z_{1}\right|^{\operatorname{ord}(\mathfrak{a}-\mathfrak{b})}\right)\right)
$$


For any section $f$ of $\operatorname{End}(E) \otimes \Omega^{1,0}$, we have the decomposition

$$
f=\sum f_{\mathfrak{a}, \mathfrak{b}}, \quad f_{\mathfrak{a}, \mathfrak{b}} \in \operatorname{Hom}\left(E_{\mathfrak{b}}, E_{\mathfrak{a}}\right) \otimes \Omega^{1,0} .
$$

From the relation $\partial_{1} \mathcal{U}-\left[\theta_{1}, \mathcal{Q}\right]+\theta_{1}=0$, we obtain the following equality for $\mathfrak{a} \neq \mathfrak{b}$ :

$$
\left(\partial_{1} \mathcal{U}\right)_{\mathfrak{a}, \mathfrak{b}}-\partial_{1}(\mathfrak{a}-\mathfrak{b}) \mathcal{Q}_{\mathfrak{a}, \mathfrak{b}}-\left(\theta_{1, \mathfrak{a}}-\partial_{1} \mathfrak{a}\right) \mathcal{Q}_{\mathfrak{a}, \mathfrak{b}}+\mathcal{Q}_{\mathfrak{a}, \mathfrak{b}}\left(\theta_{1, \mathfrak{b}}-\partial_{1} \mathfrak{b}\right)=0
$$

Note that (see Propositions 6.1 and 6.2

$$
\partial(\mathfrak{a}-\mathfrak{b}) / \partial z_{1} \sim\left|z_{1}^{\operatorname{ord}(\mathfrak{a}-\mathfrak{b})-1}\right|, \quad\left|\theta_{1, \mathfrak{a}}-\partial_{1} \mathfrak{a}\right|_{h}=O\left(d z_{1} / z_{1}\right)
$$

The estimate 7.12 implies

$$
\left|\left(\partial_{1} \mathcal{U}\right)_{\mathfrak{a}, \mathfrak{b}}\right|=O\left(\exp \left(-\epsilon_{2}\left|z_{1}\right|^{\operatorname{ord}(\mathfrak{a}-\mathfrak{b})}\right)\right) .
$$

Due to 7.13 and 7.14 , there exists $\epsilon_{3}>0$ such that, for $\mathfrak{a} \neq \mathfrak{b}$,

$$
\left|\mathcal{Q}_{\mathfrak{a}, \mathfrak{b}}\right|_{h}=O\left(\exp \left(-\epsilon_{3}\left|z_{1}\right|^{\operatorname{ord}(\mathfrak{a}-\mathfrak{b})}\right)\right) .
$$

By using 7.11, we obtain the desired estimate for $\mathcal{Q}$ with respect to $\mathcal{P}^{\left(\lambda_{0}\right)} h$, if $U\left(\lambda_{0}\right)$ is sufficiently small.

7.1.8. Complement for the normal crossing case. Let $X:=\Delta^{n}$ and $D:=$ $\bigcup_{i=1}^{\ell}\left\{z_{i}=0\right\}$. Let $\left(E, \bar{\partial}_{E}, \theta, h\right)$ be an unramifiedly good wild harmonic bundle on $X-D$. Let $\mathcal{U}$ be a holomorphic section of $\operatorname{End}(E)$ on $X-D$ such that $[\theta, \mathcal{U}]=0$. Let $\mathcal{Q}$ be a $C^{\infty}$-section of $\operatorname{End}(E)$ on $X-D$ such that $\mathcal{Q}^{\dagger}=\mathcal{Q}$. Assume that they satisfy the equations 7.2 and 7.3 . We also assume that there exists a holomorphic decomposition $(E, \theta)=\bigoplus_{\mathfrak{a} \in \operatorname{Irr}(\theta)}\left(E_{\mathfrak{a}}, \theta_{\mathfrak{a}}\right)$ such that each $\theta_{\mathfrak{a}}-d \mathfrak{a} \cdot \operatorname{id}_{E_{\mathfrak{a}}}$ is tame. Let $\pi_{\mathfrak{a}}$ denote the projection onto $E_{\mathfrak{a}}$ with respect to the above decomposition, and set $\widetilde{\mathcal{U}}:=\mathcal{U}+\sum_{\mathfrak{a} \in \operatorname{Irr}(\theta)} \mathfrak{a} \cdot \pi_{\mathfrak{a}}$.

Corollary 7.1. $\widetilde{\mathcal{U}}$ is bounded with respect to $h$.

Proof. This follows from Proposition 7.1 above and the estimate in Subsection 7.7 of 23 .

\section{$\S 7.2$. Prolongation of a variation of integrable twistor structure}

7.2.1. Statements. Let $X$ be a complex manifold, and let $D$ be a simple normal crossing divisor of $X$. Let $\left(\mathcal{E}^{\triangle}, \widetilde{\mathbb{D}}^{\triangle}, \mathcal{S}\right)$ be a variation of pure polarized integrable twistor structure of weight 0 on $\mathbb{P}^{1} \times(X-D)$. We have the underlying harmonic bundle $\left(E, \bar{\partial}_{E}, \theta, h\right)$ on $X-D$. 


\section{Definition 7.1.}

- We say that $\left(\mathcal{E}^{\triangle}, \widetilde{\mathbb{D}}^{\triangle}, \mathcal{S}\right.$ ) is tame (wild, good wild, unramifiedly good wild) if $\left(E, \bar{\partial}_{E}, \theta, h\right)$ is tame (wild, good wild, unramifiedly good wild).

- If we are given a real structure $\kappa$ of $\left(\mathcal{E}^{\triangle}, \widetilde{\mathbb{D}}^{\triangle}, \mathcal{S}\right)$, we say that the variation of polarized pure twistor-TERP structure $\left(\mathcal{E}^{\triangle}, \widetilde{\mathbb{D}}^{\triangle}, \mathcal{S}, \kappa, 0\right)$ is tame (wild, good wild, unramifiedly good wild) if $\left(\mathcal{E}^{\triangle}, \widetilde{\mathbb{D}}^{\triangle}, \mathcal{S}\right)$ is tame (wild, good wild, unramifiedly good wild).

Note that "wild" does not imply "good wild" as remarked in Remark 19 .

Assume that $\left(E, \bar{\partial}_{E}, \theta, h\right)$ is good wild. We will show the following proposition later. (The tame case was shown in [13.)

Lemma 7.3. The sets $\mathcal{K} \mathcal{M S}\left(\mathcal{P E}^{0}, i\right)$ are contained in $\mathbb{R} \times\{0\}$.

We use the notation in Subsection 2.1.7. As explained in Subsection 6.3, $(\mathcal{E}, \mathbb{D})$ is prolonged to the family of meromorphic $\lambda$-flat bundles $(\mathcal{Q E}, \mathbb{D})$ on $\mathbb{C}_{\lambda} \times(X, D)$, and $\left(\mathcal{E}^{\dagger}, \mathbb{D}^{\dagger}\right)$ is prolonged to the family of meromorphic $\mu$-flat bundles $\left(\mathcal{Q E} \mathcal{E}^{\dagger}, \mathbb{D}^{\dagger}\right)$ on $\mathbb{C}_{\mu} \times\left(X^{\dagger}, D^{\dagger}\right)$.

\section{Theorem 7.1.}

- $\widetilde{\mathbb{D}}^{f}\left(\right.$ resp. $\left.\widetilde{\mathbb{D}}^{\dagger}\right)$ gives a meromorphic flat connection of $\mathcal{Q E}\left(\right.$ resp. $\left.\mathcal{Q E}^{\dagger}\right)$.

- If a real structure $\kappa$ of $\left(\mathcal{E}^{\triangle}, \widetilde{\mathbb{D}}^{\triangle}, \mathcal{S}\right)$ is given, $\kappa_{0}: \gamma^{*} \mathcal{E}^{\dagger} \simeq \mathcal{E}$ extends to an isomorphism $\gamma^{*} \mathcal{Q E} \mathcal{E}^{\dagger} \simeq \mathcal{Q E}$. Similarly, $\kappa_{\infty}: \gamma^{*} \mathcal{E} \simeq \mathcal{E}^{\dagger}$ extends to $\gamma^{*} \mathcal{Q E} \simeq \mathcal{Q} \mathcal{E}^{\dagger}$.

For the proof of Lemma 7.3 and Theorem 7.1 , we may and will assume (i) $D$ is smooth, i.e., $\ell=1$, (ii) $\left(E, \bar{\partial}_{E}, \theta, h\right)$ is unramified.

Remark 25. Such a prolongment was studied in [13] for the tame case, which can be done without an estimate as in Proposition 7.1.

Remark 26. As a result of Lemma 7.3, $\mathfrak{p}(\lambda, \boldsymbol{u})$ for $\boldsymbol{u} \in \mathcal{K} \mathcal{M S}\left(\mathcal{P E} \mathcal{E}^{0}, i\right)$ is independent of $\lambda$. Thus, we obtain a family of good filtered $\lambda$-flat bundles $\left(\mathcal{Q}_{*} \mathcal{E}, \mathbb{D}\right)$ on $\mathbb{C}_{\lambda} \times(X, D)$. Similarly, we obtain a family of good filtered $\mu$-flat bundles $\left(\mathcal{Q}_{*} \mathcal{E}^{\dagger}, \mathbb{D}^{\dagger}\right)$ on $\mathbb{C}_{\mu} \times\left(X^{\dagger}, D^{\dagger}\right)$.

7.2.2. Meromorphic connection on $\mathcal{P}^{\left(\lambda_{0}\right)} \mathcal{E}$. Let $\lambda_{0} \in \mathbb{C}_{\lambda}$, and let $U\left(\lambda_{0}\right)$ be a small neighbourhood of $\lambda_{0}$ in $\mathbb{C}_{\lambda}$. We set $\mathcal{X}^{\left(\lambda_{0}\right)}:=U\left(\lambda_{0}\right) \times X$ and $\mathcal{D}^{\left(\lambda_{0}\right)}:=$ $U\left(\lambda_{0}\right) \times D$. Recall that we have a family of meromorphic $\lambda$-flat bundles $\left(\mathcal{P}^{\left(\lambda_{0}\right)} \mathcal{E}, \mathbb{D}\right)$ on $\left(\mathcal{X}^{\left(\lambda_{0}\right)}, \mathcal{D}^{\left(\lambda_{0}\right)}\right)$, as explained in Subsection 6.3 . Note that $\mathcal{P}^{\left(\lambda_{0}\right)} \mathcal{E}$ is identified with the sheaf of holomorphic sections of $\mathcal{E}$ of polynomial order with respect to $\mathcal{P}_{\text {irr }}^{\left(\lambda_{0}\right)} h$, 
because $\mathcal{P}_{\text {irr }}^{\left(\lambda_{0}\right)} h$ and $\mathcal{P}^{\left(\lambda_{0}\right)} h$ are mutually bounded up to polynomial orders. (See 7.4 for $\mathcal{P}_{\text {irr }}^{\left(\lambda_{0}\right)} h$. They are different in general.)

Proposition 7.3. $\widetilde{\mathbb{D}}^{f}$ gives a meromorphic flat connection on $\mathcal{P}^{\left(\lambda_{0}\right)} \mathcal{E}$.

Proof. We have only to show $\lambda^{2} \nabla_{\lambda}\left(\partial_{\lambda}\right) \mathcal{P}^{\left(\lambda_{0}\right)} \mathcal{E} \subset \mathcal{P}^{\left(\lambda_{0}\right)} \mathcal{E}$. As mentioned in Subsection 2.1.7, we have the induced holomorphic section $\mathcal{U}$ of $\operatorname{End}(E)$ on $X-D$ such that $[\theta, \mathcal{U}]=0$, and the $C^{\infty}$-section $\mathcal{Q}$ of $\operatorname{End}(E)$ such that $\mathcal{Q}^{\dagger}=\mathcal{Q}$, determined by

$$
\nabla_{\lambda}=d_{\lambda}+\left(\lambda^{-1} \mathcal{U}-\mathcal{Q}-\lambda \mathcal{U}^{\dagger}\right) \frac{d \lambda}{\lambda}
$$

where $d_{\lambda}$ denotes the naturally induced flat connection on $p_{\lambda}^{-1} E$ along the $\lambda$ direction. They satisfy the equations 7.2 and $(7.3)$.

Let $\boldsymbol{v}=\left(\boldsymbol{v}_{\mathfrak{a}}\right)$ be a holomorphic frame of $\mathcal{P}_{0} \mathcal{E}^{0}$ compatible with the decomposition $\mathcal{P}_{0} \mathcal{E}^{0}=\bigoplus_{\mathfrak{a}} \mathcal{P}_{0} \mathcal{E}_{\mathfrak{a}}^{0}$. Corresponding to the decomposition $\boldsymbol{v}=\left(\boldsymbol{v}_{\mathfrak{a}}\right)$, the identity matrix is decomposed into $\bigoplus_{\mathfrak{a} \in \operatorname{Irr}(\theta)} I_{\mathfrak{a}}$. We regard $\boldsymbol{v}$ as a $C^{\infty}$-frame of $\mathcal{E}_{\mid \mathcal{X}^{\left(\lambda_{0}\right)}-\mathcal{D}^{\left(\lambda_{0}\right)}}$, and we set

$$
\widetilde{\boldsymbol{v}}=g_{\mathrm{irr}}\left(\lambda-\lambda_{0}\right)^{-1} \boldsymbol{v}=\boldsymbol{v} \cdot\left(\bigoplus_{\mathfrak{a} \in \operatorname{Irr}(\theta)} \exp \left(-\left(\lambda-\lambda_{0}\right) \overline{\mathfrak{a}}\right) \cdot I_{\mathfrak{a}}\right) .
$$

Let $H\left(\mathcal{P}_{\text {irr }}^{\left(\lambda_{0}\right)} h, \widetilde{\boldsymbol{v}}\right)$ denote the Hermitian matrix-valued function whose $(i, j)$-entry is given by $\mathcal{P}_{\text {irr }}^{\left(\lambda_{0}\right)} h\left(\widetilde{v}_{i}, \widetilde{v}_{j}\right)$. Then it is clear that $H\left(\mathcal{P}_{\text {irr }}^{\left(\lambda_{0}\right)} h, \widetilde{\boldsymbol{v}}\right)$ and its inverse are of polynomial order. We also have the following relation:

$$
d_{\lambda} \widetilde{\boldsymbol{v}}=\widetilde{\boldsymbol{v}} \cdot A, \quad A:=-\bigoplus \overline{\mathfrak{a}} d \lambda \cdot I_{\mathfrak{a}} .
$$

Let $\boldsymbol{w}$ be a holomorphic frame of $\mathcal{P}_{a}^{\left(\lambda_{0}\right)} \mathcal{E}$. Let $H\left(\mathcal{P}_{\text {irr }}^{\left(\lambda_{0}\right)} h, \boldsymbol{w}\right)$ denote the Hermitian matrix-valued function whose $(i, j)$-entry is $\mathcal{P}_{\text {irr }}^{\left(\lambda_{0}\right)} h\left(w_{i}, w_{j}\right)$. Then $H\left(\mathcal{P}_{\text {irr }}^{\left(\lambda_{0}\right)} h, \boldsymbol{w}\right)$ and its inverse are of polynomial order. (See Subsection 9.1.2 of [23, for example.) Let $G$ be the matrix-valued function determined by $\boldsymbol{w}=\widetilde{\boldsymbol{v}} \cdot G$. Then $G$ and $G^{-1}$ are of polynomial order. We have

$$
d_{\lambda} \boldsymbol{w}=\widetilde{\boldsymbol{v}} \cdot\left(A G+d_{\lambda} G\right)=\boldsymbol{w} \cdot\left(G^{-1} A G+G^{-1} d_{\lambda} G\right)
$$

Since $\widetilde{\boldsymbol{v}}$ and $\boldsymbol{w}$ are $\lambda$-holomorphic, $G$ is $\lambda$-holomorphic. Hence, $d_{\lambda} G$ and $G^{-1} A G+$ $G^{-1} d_{\lambda} G$ are of polynomial order.

Let $B$ be determined by $\lambda^{2} \nabla_{\lambda}\left(\partial_{\lambda}\right) \boldsymbol{w}=\boldsymbol{w} \cdot B$. Then $B$ is of polynomial order due to Proposition 7.2 and hence meromorphic. Thus, the proof of Proposition 7.3 is finished. 
We have the irregular decomposition

$$
\left(\mathcal{P}_{a}^{\left(\lambda_{0}\right)} \mathcal{E}, \mathbb{D}\right)_{\mid \widehat{\mathcal{D}}^{\left(\lambda_{0}\right)}}=\bigoplus_{\mathfrak{a} \in \operatorname{Irr}(\theta)}\left(\mathcal{P}_{a}^{\left(\lambda_{0}\right)} \widehat{\mathcal{E}}_{\mathfrak{a}}, \widehat{\mathbb{D}}_{\mathfrak{a}}\right)
$$

\section{Lemma 7.4 .}

- $\lambda^{2} \nabla_{\lambda}\left(\partial_{\lambda}\right)$ preserves the decomposition 7.15 .

- If $\lambda_{0} \neq 0$, then 7.15 is the irregular decomposition for $\left(\mathcal{P}^{\left(\lambda_{0}\right)} \mathcal{E}, \widetilde{\mathbb{D}}^{f}\right)$, and $\mathcal{P}_{a}^{\left(\lambda_{0}\right)} \mathcal{E}$ is an unramifiedly good lattice of $\mathcal{P}^{\left(\lambda_{0}\right)} \mathcal{E}$.

Proof. Since this can be shown by a standard argument, we give only an outline. Let $\widehat{\boldsymbol{v}}=\left(\widehat{\boldsymbol{v}}_{\mathfrak{a}}\right)$ be a frame of $\mathcal{P}_{a}^{\left(\lambda_{0}\right)} \mathcal{E}_{\mid \widehat{\mathcal{D}}}$ compatible with the decomposition 7.15 . Let $A=\sum A_{\mathfrak{b}, \mathfrak{a}}$ be determined by $\lambda^{2} \nabla_{\lambda}\left(\partial_{\lambda}\right) \widehat{\boldsymbol{v}}=\widehat{\boldsymbol{v}} \cdot A$. For $\mathfrak{a} \neq \mathfrak{b}$, let $F_{\mathfrak{b}, \mathfrak{a}}: \mathcal{P}_{a}^{\left(\lambda_{0}\right)} \widehat{\mathcal{E}}_{\mathfrak{a}} \rightarrow$ $\mathcal{P}_{a}^{\left(\lambda_{0}\right)} \widehat{\mathcal{E}}_{\mathfrak{b}}$ be given by $F_{\mathfrak{b}, \mathfrak{a}} \widehat{\boldsymbol{v}}_{\mathfrak{a}}=\widehat{\boldsymbol{v}}_{\mathfrak{b}} \cdot A_{\mathfrak{b}, \mathfrak{a}}$. Because $\left[\lambda^{2} \nabla_{\lambda}\left(\partial_{\lambda}\right), \mathbb{D}^{f}\right]=0$, we deduce that $F_{\mathfrak{b}, \mathfrak{a}}$ is flat. However, such a flat section has to be 0 in the case $\mathfrak{b} \neq \mathfrak{a}$. Thus, we obtain the first claim.

Let us show the second claim. Let $B_{\mathfrak{a}}$ be determined by

$$
\mathbb{D}^{f}\left(z_{1} \partial_{1}\right) \widehat{\boldsymbol{v}}_{\mathfrak{a}}=\widehat{\boldsymbol{v}}_{\mathfrak{a}} \cdot\left(\left(\lambda^{-1}+\bar{\lambda}_{0}\right) \cdot z_{1} \partial_{1} \mathfrak{a}+B_{\mathfrak{a}}\right) .
$$

Then $B_{\mathfrak{a}}$ is regular. For $\mathfrak{a}=0$, the following holds:

$$
\lambda^{2} \partial_{\lambda} B_{0}+A_{0,0} B_{0}-B_{0} A_{0,0}-z_{1} \partial_{1} A_{0,0}=0 .
$$

We have the expansions $B_{0}=\sum_{m \geq 0} B_{0 ; m} z_{1}^{m}$ and $A_{0,0}=\sum_{m \geq N} A_{0,0 ; m} z_{1}^{m}$. We assume $N<0$ and $A_{0,0 ; N} \neq 0$. We obtain the relation $\left[B_{0 ; 0}, A_{0,0 ; N}\right]-N A_{0,0 ; N}=0$ on $\mathcal{D}^{\left(\lambda_{0}\right)}$. Note that the eigenvalues of $B_{0 ; 0}$ are of the form $\lambda^{-1} \mathfrak{e}(\lambda, u)$, where $u \in \mathcal{K} \mathcal{M S}\left(\mathcal{P} \mathcal{E}^{0}\right)$ and $a-1<\mathfrak{p}\left(\lambda_{0}, u\right) \leq a$. This implies that the difference of two distinct eigenvalues of $B_{0 ; 0}$ cannot be $N$. Therefore, we obtain $A_{0,0 ; N}=0$, which contradicts our assumption. Hence, $N \geq 0$.

Let $d$ denote the exterior derivative on $\mathcal{X}^{\left(\lambda_{0}\right)}$. By considering a twist with a meromorphic flat line bundle given by $\nabla e=e \cdot d\left(\left(\lambda^{-1}+\bar{\lambda}_{0}\right) \mathfrak{a}\right)$, we find that $\widetilde{\mathbb{D}}^{f}=\mathbb{D}^{f}+\nabla_{\lambda}$ on $\mathcal{P}^{\left(\lambda_{0}\right)} \mathcal{E}_{\mid \widehat{\mathcal{D}}^{\left(\lambda_{0}\right)}}$ is of the form

$$
\widetilde{\mathbb{D}}^{f}=\bigoplus_{\mathfrak{a} \in \operatorname{Irr}(\theta)}\left(d\left(\left(\lambda^{-1}+\bar{\lambda}_{0}\right) \mathfrak{a}\right)+\widetilde{\mathbb{D}}_{\mathcal{P}^{\left(\lambda_{0}\right)} \widehat{\mathcal{E}}, \mathfrak{a}}^{f}\right),
$$

where $\widetilde{\mathbb{D}}_{\mathcal{P}^{\left(\lambda_{0}\right)} \widehat{\mathcal{E}}, \mathfrak{a}}^{f}$ are logarithmic with respect to $\mathcal{P}^{\left(\lambda_{0}\right)} \widehat{\mathcal{E}}_{\mathfrak{a}}$. Thus, the proof of Lemma 7.4 is finished.

7.2.3. Proof of Lemma 7.3 and Theorem 7.1. By Lemma 7.4, the eigenvalues of $\operatorname{Res}\left(\widetilde{\mathbb{D}}^{f}\right)$ on $\mathcal{P}_{b}^{\left(\lambda_{0}\right)} \mathcal{E}_{\mid \mathcal{D}^{\left(\lambda_{0}\right)}}$ are constant. On the other hand, the eigenvalues of 
$\operatorname{Res}\left(\mathbb{D}^{f}\right)=\operatorname{Res}\left(\widetilde{\mathbb{D}}^{f}\right)$ on $\mathcal{P}_{b}^{\left(\lambda_{0}\right)} \mathcal{E}_{\mid \mathcal{D}^{\left(\lambda_{0}\right)}}$ have to be of the form $\lambda^{-1} \alpha-a-\lambda \bar{\alpha}$ for $(a, \alpha) \in \mathcal{K} \mathcal{M S}\left(\mathcal{P} \mathcal{E}^{0}\right)$ by Lemma 6.1. Hence, $\alpha=0$ for any $(a, \alpha) \in \mathcal{K} \mathcal{M S}\left(\mathcal{P} \mathcal{E}^{0}\right)$, i.e., $\mathcal{K} \mathcal{M S}\left(\mathcal{P E}^{0}\right) \subset \mathbb{R} \times\{0\}$. Thus, Lemma 7.3 is proved.

Let us show Theorem 7.1. The first claim follows from Lemma 5.19, Proposition 7.3 and the definition of $\mathcal{Q E}$ in Subsection 6.3.3. To show the second claim, we remark that $\kappa$ is flat and preserves the pluri-harmonic metrics for $\left(\mathcal{E}^{\triangle}, \mathbb{D}^{\triangle}, \mathcal{S}\right)$ and $\gamma^{*}\left(\mathcal{E}^{\triangle}, \mathbb{D}^{\triangle}, \mathcal{S}\right)$. We also remark that we have only to consider the case in which $D$ is smooth. We have $\operatorname{Irr}\left(\mathbb{D}^{\lambda}, \mathcal{Q} \mathcal{E}^{\lambda}\right)=\operatorname{Irr}(\theta)$ and $\operatorname{Irr}\left(\mathbb{D}^{\dagger} \bar{\lambda}, \mathcal{Q} \mathcal{E}^{\dagger \bar{\lambda}}\right)=\operatorname{Irr}\left(\theta^{\dagger}\right)=\{\overline{\mathfrak{a}} \mid \mathfrak{a} \in \operatorname{Irr}(\theta)\}$. Hence, we have the natural identification $\operatorname{Irr}\left(\mathbb{D}^{\lambda}, \mathcal{Q} \mathcal{E}^{\lambda}\right)=\operatorname{Irr}\left(\gamma^{*} \mathbb{D}^{\dagger} \bar{\lambda}, \gamma^{*} \mathcal{Q} \mathcal{E}^{\dagger} \bar{\lambda}\right)$. Since the full Stokes filtrations are characterized by growth order of the norms of flat sections with respect to the pluri-harmonic metrics (Proposition 6.8), the full Stokes filtrations are preserved by $\kappa$. Thus, the second claim of Theorem 7.1 follows from Lemma 5.18 .

Remark 27. Because $\mathcal{K} \mathcal{M S}\left(\mathcal{P} \mathcal{E}^{0}\right) \subset \mathbb{R} \times\{0\}$, it turns out that any $\lambda \neq 0$ is generic, which we will use implicitly.

\section{§7.3. Reduction from wild to tame}

7.3.1. Construction of the reductions. Let $X:=\Delta^{n}$ and $D:=\bigcup_{i=1}^{\ell}\left\{z_{i}=0\right\}$. Let $\left(\mathcal{E}^{\triangle}, \widetilde{\mathbb{D}}^{\triangle}, \mathcal{S}\right)$ be an unramifiedly good wild variation of pure polarized integrable twistor structure of weight 0 on $\mathbb{P}^{1} \times(X-D)$. We have the underlying harmonic bundle $\left(E, \bar{\partial}_{E}, \theta, h\right)$. We take an auxiliary sequence $\mathcal{M}=(\boldsymbol{m}(0), \boldsymbol{m}(1), \ldots, \boldsymbol{m}(L))$ for $\operatorname{Irr}(\theta)$ as in Subsection 5.2.1.

For each $\mathfrak{a} \in \overline{\operatorname{Irr}}(\theta, \boldsymbol{m}(0))$, we obtain the variation of pure polarized twistor structure $\operatorname{Gr}_{\mathfrak{a}}^{\boldsymbol{m}(0)}\left(\mathcal{E}^{\triangle}, \mathbb{D}^{\triangle}, \mathcal{S}\right)$ by taking $\mathrm{Gr}$ with respect to Stokes filtrations on the level $\boldsymbol{m}(0)$, as explained in Subsection 6.4. By Theorem 7.1 and Lemma 5.5 it is enriched to integrable $\operatorname{Gr}_{\mathfrak{a}}^{\boldsymbol{m}(0)}\left(\mathcal{E}^{\triangle}, \widetilde{\mathbb{D}}^{\triangle}, \mathcal{S}\right)$. If a real structure $\kappa$ of $\left(\mathcal{E}^{\triangle}, \mathbb{\mathbb { D }}^{\triangle}, \mathcal{S}\right)$ is given, $\kappa_{0}$ and $\kappa_{\infty}$ preserve the Stokes filtration on the level $\boldsymbol{m}(0)$, which follows from Theorem 7.1 and Lemma 5.3. Hence, we also have the induced real structure $\operatorname{Gr}_{\mathfrak{a}}^{\boldsymbol{m}(0)}(\kappa)$ of $\operatorname{Gr}_{\mathfrak{a}}^{\boldsymbol{m}(0)}\left(\mathcal{E}^{\triangle}, \widetilde{\mathbb{D}}^{\triangle}, \mathcal{S}\right)$, and we obtain a pure polarized variation of twistor-TERP structure $\operatorname{Gr}_{\mathfrak{a}}^{\boldsymbol{m}(0)}\left(\mathcal{E} \triangle, \widetilde{\mathbb{D}}^{\triangle}, \mathcal{S}, \kappa, 0\right)$ for each $\mathfrak{a} \in \overline{\operatorname{Irr}}(\mathbb{D}, \boldsymbol{m}(0))$.

Applying the above procedure inductively, $\operatorname{Gr}_{\mathfrak{a}}^{\boldsymbol{m}(j)}\left(\mathcal{E}^{\triangle}, \mathbb{D}^{\triangle}, \mathcal{S}\right)$ are enriched to integrable $\operatorname{Gr}_{\mathfrak{a}}^{\boldsymbol{m}(j)}\left(\mathcal{E}^{\triangle}, \widetilde{\mathbb{D}}^{\triangle}, \mathcal{S}\right)$ for any $\mathfrak{a} \in \overline{\operatorname{Irr}}(\theta, \boldsymbol{m}(j))$. (See the argument in Subsection 6.4.) If a real structure $\kappa$ is provided, the reductions are also equipped with induced real structures, and we obtain a variation of twistor-TERP structure $\operatorname{Gr}_{\mathfrak{a}}^{\boldsymbol{m}(j)}\left(\mathcal{E}^{\triangle}, \widetilde{\mathbb{D}}^{\triangle}, \mathcal{S}, \kappa, 0\right)$. In the case $\boldsymbol{m}(L)$, we use the symbols $\operatorname{Gr}_{\mathfrak{a}}^{\text {full }}\left(\mathcal{E}^{\triangle}, \widetilde{\mathbb{D}}^{\triangle}, \mathcal{S}\right)$ and $\operatorname{Gr}_{\mathfrak{a}}^{\text {full }}\left(\mathcal{E}^{\triangle}, \widetilde{\mathbb{D}}^{\triangle}, \mathcal{S}, \kappa, 0\right)$. They are called the full reductions.

For any $\mathfrak{a} \in \operatorname{Irr}(\theta)$, we have the harmonic bundles $L(-\mathfrak{a})$ as in Subsection 6.4 The associated variation of polarized pure twistor structure is also denoted by the 
same symbol $L(-\mathfrak{a})$. As explained in Subsection 2.2.1, it is naturally enriched to a variation of pure twistor-TERP structure of weight 0 . The underlying harmonic bundle of $\operatorname{Gr}_{\mathfrak{a}}^{\text {full }}\left(\mathcal{E}^{\triangle}, \widetilde{\mathbb{D}}^{\triangle}, \mathcal{S}\right) \otimes L(-\mathfrak{a})$ is tame for each $\mathfrak{a} \in \operatorname{Irr}(\theta)$. This procedure is the reduction "from wild to tame" in the integrable case. We have a similar reduction in the twistor-TERP case.

7.3.2. Approximating map and estimate of the new supersymmetric index. Let $\left(\mathcal{E}^{\triangle}, \widetilde{\mathbb{D}}^{\triangle}, \mathcal{S}\right)$ and $\left(E, \bar{\partial}_{E}, \theta, h\right)$ be as above. Let $\bar{\partial}_{\mathbb{P}^{1}, \mathcal{E}} \triangle$ denote the $\lambda$ holomorphic structure of $\mathcal{E}^{\triangle}$.

One step reduction. By the one step reduction in Subsection 7.3.1, we have obtained the unramifiedly good wild variation of polarized pure integrable twistor structure

$$
\left(\mathcal{E}_{0}^{\triangle}, \widetilde{\mathbb{D}}_{0}^{\triangle}, \mathcal{S}_{0}\right):=\bigoplus_{\mathfrak{a} \in \overline{\operatorname{Irr}}(\theta, \boldsymbol{m}(0))} \operatorname{Gr}_{\mathfrak{a}}^{\boldsymbol{m}(0)}\left(\mathcal{E}^{\triangle}, \widetilde{\mathbb{D}}^{\triangle}, \mathcal{S}\right) .
$$

Let $\left(E_{0}, \bar{\partial}_{E_{0}}, \theta_{0}, h_{0}\right)$ be the underlying harmonic bundle. Let $\bar{\partial}_{\mathbb{P}^{1}, \mathcal{E}_{0}}$ denote the $\lambda$ holomorphic structure of $\mathcal{E}_{0}^{\triangle}$. We fix a hermitian metric $g_{\mathbb{P}^{1}}$ on $\Omega_{\mathbb{P}^{1}}^{0,1} \oplus \Omega_{\mathbb{P}^{1}}^{1,0}(2\{0, \infty\})$. We will prove the following proposition in Subsection 7.3.3.

Theorem 7.2. There exists a $C^{\infty}$-map $\Phi: \mathcal{E}_{0}^{\triangle} \rightarrow \mathcal{E}^{\triangle}$ such that the following holds for some $\epsilon>0$ with respect to $h_{0}$ and $g_{\mathbb{P}^{1}}$ :

$$
\begin{aligned}
\Phi^{*} \mathcal{S}-\mathcal{S}_{0} & =O\left(\exp \left(-\epsilon\left|\boldsymbol{z}^{\boldsymbol{m}(0)}\right|\right)\right), \\
\bar{\partial}_{\mathbb{P}^{1}, \mathcal{E}_{0}}\left(\Phi^{*} \mathcal{S}-\mathcal{S}_{0}\right) & =O\left(\exp \left(-\epsilon\left|\boldsymbol{z}^{\boldsymbol{m}(0)}\right|\right)\right), \\
\Phi^{*} \nabla_{\lambda}-\nabla_{\lambda, 0} & =O\left(\exp \left(-\epsilon\left|\boldsymbol{z}^{\boldsymbol{m}(0)}\right|\right)\right) .
\end{aligned}
$$

In fact, the estimates can be improved to $O\left(\exp \left(-\epsilon\left(|\lambda|+\left|\lambda^{-1}\right|\right)\left|\boldsymbol{z}^{\boldsymbol{m}(0)}\right|\right)\right)$. We give a consequence. Let $\mathcal{Q}_{0}$ denote the new supersymmetric index of $\left(\mathcal{E}_{0}^{\triangle}, \widetilde{\mathbb{D}}_{0}^{\triangle}, \mathcal{S}_{0}\right)$.

Corollary 7.2. We have the following estimates for some $\epsilon>0$ with respect to $h_{0}$ :

$$
\left|\Phi^{*} h-h_{0}\right|_{h_{0}}=O\left(\exp \left(-\epsilon\left|\boldsymbol{z}^{\boldsymbol{m}(0)}\right|\right)\right), \quad\left|\Phi^{*} \mathcal{Q}-\mathcal{Q}_{0}\right|_{h_{0}}=O\left(\exp \left(-\epsilon\left|\boldsymbol{z}^{\boldsymbol{m}(0)}\right|\right)\right) .
$$

Proof. This follows from Lemma 2.9.

Full reduction. By taking the full reduction in Subsection 7.3.1, we have obtained the unramifiedly good wild variation of polarized pure integrable twistor structure

$$
\left(\mathcal{E}_{1}^{\triangle}, \widetilde{\mathbb{D}}_{1}^{\triangle}, \mathcal{S}_{1}\right):=\bigoplus_{\mathfrak{a} \in \overline{\operatorname{Irr}}(\theta)} \operatorname{Gr}_{\mathfrak{a}}^{\text {full }}\left(\mathcal{E}^{\triangle}, \widetilde{\mathbb{D}}^{\triangle}, \mathcal{S}\right) .
$$

Let $\left(E_{1}, \bar{\partial}_{E_{1}}, \theta_{1}, h_{1}\right)$ be the underlying harmonic bundle, and let $\mathcal{Q}_{1}$ denote the 
new supersymmetric index for $\left(\mathcal{E}_{1}^{\triangle}, \mathbb{D}_{1}^{\triangle}\right)$. By applying Theorem 7.2 and Corollary 7.2 inductively (see Subsection 6.4 for an inductive use), we obtain a $C^{\infty}$-map $\Phi_{1}: \mathcal{E}_{1}^{\triangle} \rightarrow \mathcal{E}^{\triangle}$ such that the following holds for some $\epsilon>0$ with respect to $h_{1}$ :

$$
\left|\Phi_{1}^{*} h-h_{1}\right|_{h_{1}}=O\left(\exp \left(-\epsilon\left|\boldsymbol{z}^{\boldsymbol{m}(L)}\right|\right)\right), \quad\left|\Phi_{1}^{*} \mathcal{Q}-\mathcal{Q}_{1}\right|_{h_{1}}=O\left(\exp \left(-\epsilon\left|\boldsymbol{z}^{\boldsymbol{m}(L)}\right|\right)\right) .
$$

Note that the new supersymmetric index is unchanged after taking the tensor product with $L(-\mathfrak{a})$. (See Subsection 2.2.1.) Hence, the study of the asymptotic behaviour of the new supersymmetric index reduces to the study in the tame case, up to decay with exponential orders.

7.3.3. Construction of an approximating map. We assume that the coordinate is as in Remark 15 for the good set $\operatorname{Irr}(\theta)$. Let $k$ be determined by $\boldsymbol{m}(0) \in \mathbb{Z}_{<0}^{k} \times \mathbf{0}_{\ell-k}$. Let $\lambda_{0} \in \mathbb{C}_{\lambda}$. Let $U\left(\lambda_{0}\right)$ denote a small neighbourhood of $\lambda_{0}$. We set $\mathcal{X}^{\left(\lambda_{0}\right)}:=U\left(\lambda_{0}\right) \times X$ and $\mathcal{D}^{\left(\lambda_{0}\right)}(\leq k):=U\left(\lambda_{0}\right) \times D(\leq k)$. We also use the symbol $\mathcal{D}_{i}^{\left(\lambda_{0}\right)}$ in a similar meaning. We set $W:=\mathcal{D}^{\left(\lambda_{0}\right)}(\leq k)$ if $\lambda_{0} \neq 0$, and $W:=\mathcal{D}^{\left(\lambda_{0}\right)}(\leq k) \cup(\{0\} \times X)$. Let $\sigma: \mathbb{C}_{\lambda} \rightarrow \mathbb{C}_{\mu}$ be given by $\sigma(\lambda)=-\bar{\lambda}$, which induces an anti-holomorphic map $\mathbb{C}_{\lambda} \times X \rightarrow \mathbb{C}_{\mu} \times X^{\dagger}$. We set $\mathcal{X}^{\dagger\left(-\bar{\lambda}_{0}\right)}:=\sigma\left(\mathcal{X}^{\left(\lambda_{0}\right)}\right)$.

From $\left(E, \bar{\partial}_{E}, \theta, h\right)$, we obtain the vector bundle $\mathcal{P}_{0}^{\left(\lambda_{0}\right)} \mathcal{E}$ on $\mathcal{X}^{\left(\lambda_{0}\right)}$ with a meromorphic flat connection $\widetilde{\mathbb{D}}^{f}:=\mathbb{D}^{f}+\nabla_{\lambda}$. Similarly we obtain $\mathcal{P}_{0}^{\left(\lambda_{0}\right)} \mathcal{E}_{0}$ with $\widetilde{\mathbb{D}}_{0}^{f}=$ $\mathbb{D}_{0}^{f}+\nabla_{\lambda, 0}$ from $\left(E_{0}, \bar{\partial}_{E_{0}}, \theta_{0}, h_{0}\right)$.

We also obtain the vector bundle $\mathcal{P}_{0}^{\left(\mu_{0}\right)} \mathcal{E}^{\dagger}$ with the meromorphic flat connection $\widetilde{\mathbb{D}}^{\dagger f}=\mathbb{D}^{\dagger f}+\nabla_{\mu}$ on $\mathcal{X}^{\left(\mu_{0}\right)}$ from $\left(E, \bar{\partial}_{E}, \theta, h\right)$, and the vector bundle $\mathcal{P}_{0}^{\left(\mu_{0}\right)} \mathcal{E}_{0}^{\dagger}$ with the meromorphic flat connection $\widetilde{\mathbb{D}}_{0}^{\dagger f}=\mathbb{D}_{0}^{\dagger f}+\nabla_{\mu, 0}$ from $\left(E_{0}, \bar{\partial}_{E_{0}}, \theta_{0}, h_{0}\right)$.

Let $\mathbb{D}_{\leq k}$ denote the restriction of $\mathbb{D}$ to the $\left(z_{1}, \ldots, z_{k}\right)$-direction.

Preliminaries. Let $S$ be a small multi-sector of $\mathcal{X}^{\left(\lambda_{0}\right)}-W$. By Proposition 5.2 . we can take a $\mathbb{D}_{\leq k}$-flat splitting

$$
\mathcal{P}_{0}^{\left(\lambda_{0}\right)} \mathcal{E}_{\mid \bar{S}}=\bigoplus_{\mathfrak{a} \in \operatorname{Irr}(\theta)} \mathcal{P}_{0}^{\left(\lambda_{0}\right)} \mathcal{E}_{\mathfrak{a}, S}
$$

of the Stokes filtration on the level $\boldsymbol{m}(0)$ such that the restrictions to $\mathcal{D}_{j}^{\left(\lambda_{0}\right)} \cap S$ $(j=k+1, \ldots, \ell)$ are compatible with $\operatorname{Res}_{j}(\mathbb{D})$ and the filtrations ${ }^{j} F^{\left(\lambda_{0}\right)}$. If $\lambda_{0} \neq 0$, we may assume that it is $\mathbb{D}^{f}$-flat by Proposition 5.3 and Lemma 7.3 . (Note that the $\mathbb{D}^{f}$-flatness implies the compatibility with the residues and the parabolic filtrations.) By construction of $\mathrm{Gr}^{\boldsymbol{m}(0)}$, it induces an isomorphism $\left(\mathcal{P}_{0}^{\left(\lambda_{0}\right)} \mathcal{E}_{0}, \mathbb{D}_{0, \leq k}\right)_{\mid \bar{S}} \stackrel{\sim}{\rightarrow}$ $\left(\mathcal{P}_{0}^{\left(\lambda_{0}\right)} \mathcal{E}, \mathbb{D}_{\leq k}\right)_{\mid \bar{S}}$. Let $\Phi_{S}^{p}(p=0, \ldots, m)$ be such isomorphisms. Let $a_{p}(p=0, \ldots, m)$ be non-negative $C^{\infty}$-functions on $S$ such that (i) $\sum a_{p}=1$, (ii) $\partial_{i} a_{p}$ and $\partial_{\lambda} a_{p}$ are $O\left(|\lambda|^{-C} \prod_{i=1}^{k}\left|z_{i}\right|^{-C}\right)$ for some $C>0$. We set $\Phi_{S}:=\sum a_{p} \Phi_{S}^{p}$. We also set $G:=\left(\Phi_{S}^{0}\right)^{-1} \circ \Phi_{S}$ and $G^{p}:=\left(\Phi_{S}^{0}\right)^{-1} \circ \Phi_{S}^{p}$. 
Lemma 7.5. We have the following estimates with respect to $h_{0}$ for some $\epsilon>0$ :

$$
\begin{gathered}
G^{p}-\mathrm{id}=O\left(\exp \left(-\epsilon\left|\lambda^{-1} z^{\boldsymbol{m}(0)}\right|\right)\right), \\
\left(\Phi_{S}^{0}\right)^{-1} \circ\left(\lambda^{2} \nabla_{\lambda}\left(\partial_{\lambda}\right)\right) \circ \Phi_{S}^{0}-\lambda^{2} \nabla_{\lambda, 0}\left(\partial_{\lambda}\right)=O\left(\exp \left(-\epsilon\left|\lambda^{-1} z^{\boldsymbol{m}(0)}\right|\right)\right) .
\end{gathered}
$$

Proof. Let $\mathcal{G}$ be the left hand side of 7.18 or 7.19 . It is flat with respect to $\mathbb{D}_{0, \leq k}$, and strictly decreases the Stokes filtration on the level $\boldsymbol{m}(0)$. Moreover, $\mathcal{G}_{\mid \mathcal{D}_{i}^{\left(\lambda_{0}\right)} \cap S}$ preserves the filtrations ${ }^{i} F^{\left(\lambda_{0}\right)}$ and the residues $\operatorname{Res}_{j}(\mathbb{D})$ for $j=k+1, \ldots, \ell$. Then we obtain the desired estimate by using the estimate in Subsection 9.3 of [23]. (It is also easy to show it directly.)

Hence, we have $\mid G-$ id $\left.\right|_{h_{0}}=O\left(\exp \left(-\epsilon\left|\lambda^{-1} \boldsymbol{z}^{\boldsymbol{m}(0)}\right|\right)\right)$. We set $\Phi_{S}^{*} \nabla_{\lambda}\left(\partial_{\lambda}\right):=$ $\Phi_{S}^{-1} \circ\left(\nabla_{\lambda}\left(\partial_{\lambda}\right)\right) \circ \Phi_{S}$. We use the symbol $\left(\Phi_{S}^{0}\right)^{*} \nabla_{\lambda}\left(\partial_{\lambda}\right)$ in a similar meaning. By the previous lemma, we have the following estimate for some $\epsilon>0$ with respect to $h_{0}$ :

$$
\left(\Phi_{S}^{0}\right)^{*} \nabla_{\lambda}\left(\partial_{\lambda}\right)-\nabla_{\lambda, 0}\left(\partial_{\lambda}\right)=O\left(\exp \left(-\epsilon\left|\lambda^{-1} z^{\boldsymbol{m}(0)}\right|\right)\right) .
$$

Lemma 7.6. The following estimate holds for some $\epsilon>0$ with respect to $h_{0}$ :

$$
\Phi_{S}^{*} \nabla_{\lambda}\left(\partial_{\lambda}\right)-\nabla_{\lambda, 0}\left(\partial_{\lambda}\right)=O\left(\exp \left(-\epsilon\left|\lambda^{-1} \boldsymbol{z}^{\boldsymbol{m}(0)}\right|\right)\right) .
$$

Proof. We have the following equalities:

$$
\begin{aligned}
& \Phi_{S}^{*} \nabla_{\lambda}\left(\partial_{\lambda}\right)-\nabla_{\lambda, 0}\left(\partial_{\lambda}\right) \\
= & \left(\Phi_{S}^{-1} \circ \Phi_{S}^{0}\right) \circ\left(\Phi_{S}^{0}\right)^{*} \nabla_{\lambda}\left(\partial_{\lambda}\right) \circ\left(\left(\Phi_{S}^{0}\right)^{-1} \circ \Phi_{S}\right)-\nabla_{\lambda, 0}\left(\partial_{\lambda}\right) \\
= & G^{-1} \circ\left(\left(\Phi_{S}^{0}\right)^{*} \nabla_{\lambda}\left(\partial_{\lambda}\right)-\nabla_{\lambda, 0}\left(\partial_{\lambda}\right)\right) \circ G+G^{-1} \circ \nabla_{\lambda, 0}\left(\partial_{\lambda}\right) \circ G-\nabla_{\lambda, 0}\left(\partial_{\lambda}\right) \\
= & G^{-1} \circ\left(\left(\Phi_{S}^{0}\right)^{*} \nabla_{\lambda}\left(\partial_{\lambda}\right)-\nabla_{\lambda, 0}\left(\partial_{\lambda}\right)\right) \circ G+G^{-1} \cdot\left(\nabla_{\lambda, 0}\left(\partial_{\lambda}\right) G\right) .
\end{aligned}
$$

Moreover,

$$
\nabla_{\lambda, 0}\left(\partial_{\lambda}\right) G=\sum \frac{\partial a_{p}}{\partial \lambda} \cdot G^{p}=\sum \frac{\partial a_{p}}{\partial \lambda} \cdot\left(G^{p}-\mathrm{id}\right)=O\left(\exp \left(-\epsilon\left|\lambda^{-1} \boldsymbol{z}^{\boldsymbol{m}(0)}\right|\right)\right) .
$$

Thus, we obtain the conclusion.

Assume we are also given morphisms on multi-sectors $\sigma(S)$ of $\mathcal{X}^{\dagger\left(-\bar{\lambda}_{0}\right)}-W^{\dagger}$,

$$
\Phi_{\sigma(S)}^{\dagger q}:\left(\mathcal{P}^{\left(-\bar{\lambda}_{0}\right)} \mathcal{E}_{0}^{\dagger}, \mathbb{D}_{0}^{\dagger}\right)_{\mid \sigma(\bar{S})} \rightarrow\left(\mathcal{P}^{\left(-\bar{\lambda}_{0}\right)} \mathcal{E}^{\dagger}, \mathbb{D}^{\dagger}\right)_{\mid \sigma(\bar{S})} \quad\left(q=0, \ldots, m^{\prime}\right),
$$

induced by $\mathbb{D}_{\leq k}^{\dagger}$-flat splittings of the Stokes filtration on the level $\boldsymbol{m}(0)$ such that the restriction to $\sigma(S) \cap \mathcal{D}_{j}^{\dagger\left(-\bar{\lambda}_{0}\right)}(j=k+1, \ldots, \ell)$ is compatible with the residue $\operatorname{Res}_{j}\left(\mathbb{D}^{\dagger}\right)$ and the filtration ${ }^{j} F^{\left(-\bar{\lambda}_{0}\right)}$. If $\lambda_{0} \neq 0$, we may assume that the splittings are $\mathbb{D}^{\dagger}$-flat. Let $b_{q}\left(q=0, \ldots, m^{\prime}\right)$ be non-negative $C^{\infty}$-functions on $\sigma(S)$ satisfying similar conditions for $a_{p}$. We set $\Phi_{\sigma(S)}^{\dagger}:=\sum b_{q} \Phi_{\sigma(S)}^{\dagger q}$. 
Lemma 7.7. Set $H:=\mathcal{S} \circ\left(\Phi_{S} \otimes \sigma^{*} \Phi_{\sigma(S)}^{\dagger}\right)-\mathcal{S}_{0}$. Then we have the following estimates with respect to $h_{0}$ for some $\epsilon>0$ :

$$
H=O\left(\exp \left(-\epsilon\left|\lambda^{-1} \boldsymbol{z}^{\boldsymbol{m}(0)}\right|\right)\right), \quad \bar{\partial}_{\mathcal{E}_{0}^{\triangle}, \mathbb{P}^{1}} H=O\left(\exp \left(-\epsilon\left|\lambda^{-1} \boldsymbol{z}^{\boldsymbol{m}(0)}\right|\right)\right) .
$$

Proof. We set $H_{p, q}:=\mathcal{S} \circ\left(\Phi_{S}^{p} \otimes \sigma^{*} \Phi_{\sigma(S)}^{\dagger q}\right)-\mathcal{S}_{0}$. According to an estimate in Subsection 11.4.2, we have

$$
H_{p, q}=O\left(\exp \left(-\epsilon\left|\lambda^{-1} \boldsymbol{z}^{\boldsymbol{m}(0)}\right|\right)\right)
$$

with respect to $h_{0}$ for some $\epsilon>0$. We also have $\bar{\partial}_{\mathcal{E}_{0}^{\triangle}, \mathbb{P}^{1}} H_{p, q}=0$. Thus, the claim follows.

Construction. We take a compact region $\mathcal{K}$ of $\mathbb{C}_{\lambda}$ such that the union of the interior parts of $\mathcal{K}$ and $\sigma(\mathcal{K})$ covers $\mathbb{P}^{1}$. We take a covering of

$$
(\mathcal{K} \times X)-((\mathcal{K} \times D(\leq k)) \cup(\{0\} \times X))
$$

by multi-sectors $S_{i}(i=1, \ldots, N)$ such that $S_{i}$ are sufficiently small as in Preliminaries above. Then $\mathbb{P}^{1}=\bigcup S_{i} \cup \bigcup \sigma\left(S_{i}\right)$. We take a partition $\left(\chi_{S_{i}}, \chi_{\sigma\left(S_{i}\right)} \mid\right.$ $i=1, \ldots, N)$ of unity on $\mathbb{P}^{1}$ subordinated to this covering. We assume that $\partial_{j} \chi_{S_{i}}$ and $\partial_{\lambda} \chi_{S_{i}}$ are $O\left(|\lambda|^{-C} \cdot \prod_{i=1}^{k}\left|z_{i}\right|^{-C}\right)$ for some $C>0$. We assume similar conditions for $\partial_{j} \chi_{\sigma\left(S_{i}\right)}$ and $\partial_{\mu} \chi_{\sigma\left(S_{i}\right)}$.

For each $S_{i} \subset \mathcal{X}^{\left(\lambda_{0}\right)}-W$, we take isomorphisms

$$
\begin{aligned}
& \Phi_{S_{i}}:\left(\mathcal{P}_{0}^{\left(\lambda_{0}\right)} \mathcal{E}_{0}, \mathbb{D}_{0}\right)_{\mid \bar{S}_{i}} \simeq\left(\mathcal{P}_{0}^{\left(\lambda_{0}\right)} \mathcal{E}, \mathbb{D}\right)_{\mid \bar{S}_{i}} \\
& \Phi_{\sigma\left(S_{i}\right)}^{\dagger}:\left(\mathcal{P}_{0}^{\left(-\bar{\lambda}_{0}\right)} \mathcal{E}_{0}^{\dagger}, \mathbb{D}_{0}^{\dagger}\right)_{\mid \sigma\left(\bar{S}_{i}\right)} \simeq\left(\mathcal{P}_{0}^{\left(-\bar{\lambda}_{0}\right)} \mathcal{E}^{\dagger}, \mathbb{D}^{\dagger}\right)_{\mid \sigma\left(\bar{S}_{i}\right)}
\end{aligned}
$$

induced by $\mathbb{D}_{\leq k}$-flat or $\mathbb{D}_{\leq k}^{\dagger}$-flat splittings of Stokes filtrations as above. If $\lambda_{0} \neq 0$, we assume $\mathbb{D}^{f}$-flatness and $\mathbb{D}^{\dagger f}$-flatness. We set

$$
\Phi:=\sum_{i=1}^{N} \chi_{S_{i}} \Phi_{S_{i}}+\sum_{i=1}^{N} \chi_{\sigma\left(S_{i}\right)} \Phi_{\sigma\left(S_{i}\right)} .
$$

It is easy to check that $\Phi$ satisfies the desired estimates 7.16 and (7.17), by using Lemmas 7.6 and 7.7. Note that a $\mathbb{D}$-flat splitting of the Stokes filtration of $\mathcal{P}^{\left(\lambda_{0}\right)} \mathcal{E}_{\mid \bar{S}}$ on the level $\boldsymbol{m}(0)$ naturally gives a $\mathbb{D}^{\dagger}$-flat splitting of the Stokes filtration of $\mathcal{P}^{\left(\lambda_{0}^{-1}\right)} \mathcal{E}_{\mid \bar{S}^{\prime}}^{\dagger}$ on the level $\boldsymbol{m}(0)$, where $S^{\prime}$ is the corresponding multi-sector of $\mathcal{X}^{\dagger\left(\lambda_{0}^{-1}\right)}-W^{\dagger}$, which follows from the characterization of the Stokes filtrations by the growth order of the norms of flat sections. Thus, we obtain Theorem 7.2 . 


\section{§7.4. Reduction from tame to twistor nilpotent orbit}

7.4.1. Reduction. Let $X:=\Delta^{n}, D_{i}:=\left\{z_{i}=0\right\}, D:=\bigcup_{i=1}^{\ell} D_{i}$ and $D_{\underline{\ell}}=$ $\bigcap_{i=1}^{\ell} D_{i}$. Let $\left(\mathcal{E}^{\triangle}, \widetilde{\mathbb{D}}^{\Delta}, \mathcal{S}\right)$ be a tame variation of pure polarized integrable twistor structure of weight 0 on $\mathbb{P}^{1} \times(X-D)$. We have the underlying harmonic bundle $\left(E, \bar{\partial}_{E}, \theta, h\right)$. As explained in Subsection 6.5, we have the limiting polarized mixed twistor structure $\left(S_{\boldsymbol{a}, 0}^{\mathrm{can}}(E), \boldsymbol{N}, \mathcal{S}_{\boldsymbol{a}, 0}\right)$ associated to $\left(E, \bar{\partial}_{E}, \theta, h\right)$. We also have the variation of polarized mixed twistor structure $\left(\underline{\ell}_{\boldsymbol{a}, 0}^{\triangle}, \boldsymbol{N}^{\triangle}, \mathbb{D}_{\boldsymbol{a}, 0}^{\Delta}, \mathcal{S}_{\boldsymbol{a}, 0}\right)$ of weight 0 in $\ell$ variables. Hertling and Sevenheck observed the following (see [13]).

Proposition 7.4. $\left(S_{\boldsymbol{a}, 0}^{\mathrm{can}}(E), \boldsymbol{N}, \mathcal{S}_{\boldsymbol{a}, 0}\right)$ can be naturally enriched to an integrable one $\left(S_{\boldsymbol{a}, 0}^{\mathrm{can}}(E), \nabla, \boldsymbol{N}, \mathcal{S}_{\boldsymbol{a}, 0}\right)$. Similarly, $\left(\underline{\ell}_{\boldsymbol{a}, 0}^{\triangle}, \boldsymbol{N}^{\triangle}, \mathbb{D}_{\boldsymbol{a}, 0}^{\triangle}, \mathcal{S}_{\boldsymbol{a}, 0}\right)$ can be naturally enriched to an integrable one $\left(\underline{\varepsilon}_{\boldsymbol{a}, 0}^{\Delta}, \boldsymbol{N}^{\Delta}, \widetilde{\mathbb{D}}_{\boldsymbol{a}, 0}^{\Delta}, \mathcal{S}_{\boldsymbol{a}, 0}\right)$.

If $\left(\mathcal{E}^{\triangle}, \widetilde{\mathbb{D}}^{\triangle}, \mathcal{S}\right)$ has a real structure $\kappa$, the enrichments are also equipped with induced real structures.

7.4.2. Approximating maps. For $0<R<1$, we set

$$
\begin{aligned}
X^{*}(R) & :=\left\{\left(z_{1}, \ldots, z_{n}\right)|0<| z_{i} \mid<R, i=1, \ldots, n\right\}, \\
D_{\underline{\ell}}(R) & :=\left\{\left(z_{\ell+1}, \ldots, z_{n}\right)|| z_{i} \mid<R\right\} .
\end{aligned}
$$

By the natural projection $X^{*}(R) \rightarrow D_{\underline{\ell}}(R)$, we regard $X^{*}(R)$ as $D_{\underline{\ell}}(R) \times$ $\left\{\left(z_{1}, \ldots, z_{\ell}\right)|0<| z_{i} \mid<R\right\}$. Due to Theorem 4.1. we have the integrable twistor nilpotent orbit $\operatorname{TNIL}\left(\mathcal{E}_{\boldsymbol{a}, 0}^{\Delta}, \widetilde{\mathbb{D}}_{\boldsymbol{a}, 0}^{\Delta}, \boldsymbol{N}, \mathcal{S}_{\boldsymbol{a}, 0}\right)$ on $X^{*}(R)$ for some $R$. Thus, we obtain a tame variation of pure polarized integrable twistor structure:

$$
\left(\mathcal{E}_{0}^{\triangle}, \widetilde{\mathbb{D}}_{0}^{\triangle}, \mathcal{S}_{0}\right):=\bigoplus_{\boldsymbol{a} \in \mathcal{P} \operatorname{ar}\left(\mathcal{P}_{0} \mathcal{E}^{0}, \underline{\ell}\right)} \operatorname{TNIL}\left(\mathcal{E}_{\boldsymbol{a}, 0}^{\triangle}, \widetilde{\mathbb{D}}_{\boldsymbol{a}, 0}^{\triangle}, \boldsymbol{N}, \mathcal{S}_{\boldsymbol{a}, 0}\right) \otimes L(\boldsymbol{a}) .
$$

(See Subsection 2.2.2 for $L(\boldsymbol{a})$.) We have the underlying tame harmonic bundle

$$
\left(E_{0}, \bar{\partial}_{E_{0}}, \theta_{0}, h_{0}\right)=\bigoplus\left(E_{\boldsymbol{a}}, \bar{\partial}_{\boldsymbol{a}}, \theta_{\boldsymbol{a}}, h_{\boldsymbol{a}}\right) .
$$

We would like to explain that we can approximate the original $\left(\mathcal{E}^{\triangle}, \widetilde{\mathbb{D}}^{\triangle}, \mathcal{S}\right)$ with $\left(\mathcal{E}_{0}^{\triangle}, \widetilde{\mathbb{D}}_{0}^{\triangle}, \mathcal{S}_{0}\right)$.

Let $\bar{\partial}_{\mathbb{P}^{1}, \mathcal{E}_{0}}$ denote the $\lambda$-holomorphic structure of $\mathcal{E}_{0}^{\Delta}$. We fix a hermitian metric $g_{\mathbb{P}^{1}}$ on $\Omega_{\mathbb{P}^{1}}^{0,1} \oplus \Omega_{\mathbb{P}^{1}}^{1,0}(2\{0, \infty\})$. For a permutation $\sigma$ of $\{1, \ldots, \ell\}$ and for $C>0$, we set

$$
Z(\sigma, C):=\left\{\left.\left(z_{1}, \ldots, z_{n}\right) \in X^{*}(R)|| z_{\sigma(i-1)}\right|^{C}<\left|z_{\sigma(i)}\right|, i=1, \ldots, \ell-1\right\} .
$$

If we take a sufficiently large $C>0$, we have $X^{*}(R)=\bigcup_{\sigma} Z(\sigma, C)$. For any $\epsilon>0$, we set $\Lambda_{0}(\epsilon):=\sum_{i=1}^{\ell}\left|z_{i}\right|^{\epsilon}$. We will prove the following statement in Subsection 7.4.3. 
Theorem 7.3. There exists a $C^{\infty}{ }_{-}$map $\Phi_{\sigma}: \mathcal{E}_{0}^{\triangle} \rightarrow \mathcal{E}^{\triangle}$ such that the following estimates hold for some $\epsilon>0$ with respect to $h_{0}$ and $g_{\mathbb{P}^{1}}$ on $\mathbb{P}^{1} \times Z(\sigma, C)$ :

$$
\begin{gathered}
\Phi_{\sigma}^{*} \mathcal{S}-\mathcal{S}_{0}=O\left(\Lambda_{0}(\epsilon)\right), \quad \bar{\partial}_{\mathbb{P}^{1}, \mathcal{E}_{0}^{\Delta}}\left(\Phi_{\sigma}^{*} \mathcal{S}-\mathcal{S}_{0}\right)=O\left(\Lambda_{0}(\epsilon)\right), \\
\Phi_{\sigma}^{*} \nabla_{\lambda}-\nabla_{\lambda, 0}=O\left(\Lambda_{0}(\epsilon)\right) .
\end{gathered}
$$

Before going into the proof, we give a consequence. Let $\mathcal{Q}_{0}$ and $\mathcal{Q}$ denote the new supersymmetric indices of $\left(\mathcal{E}_{0}^{\triangle}, \widetilde{\mathbb{D}}_{0}^{\triangle}\right)$ and $\left(\mathcal{E}^{\triangle}, \widetilde{\mathbb{D}}^{\triangle}\right)$. By using Lemma 2.9 we obtain the following estimates on $Z(\sigma, C)$ for some $\epsilon>0$ with respect to $h_{0}$ :

$$
\left|\Phi_{\sigma}^{*} h-h_{0}\right|_{h_{0}}=O\left(\Lambda_{0}(\epsilon)\right), \quad\left|\Phi_{\sigma}^{*} \mathcal{Q}-\mathcal{Q}_{0}\right|_{h_{0}}=O\left(\Lambda_{0}(\epsilon)\right) .
$$

Corollary 7.3. The eigenvalues of $\mathcal{Q}$ and $\mathcal{Q}_{0}$ are equal up to $O\left(\Lambda_{0}(\epsilon)\right)$ for some $\epsilon>0$.

Proof. By using $(7.22)$, we obtain the estimate on $Z(\sigma, C)$. Because $X^{*}(R)=$ $\cup Z(\sigma, C)$, the claim of the corollary follows.

We also give a more rough but global estimate, for which the proof is much simpler. For $M>0$ and $\epsilon>0$, we set

$$
\Lambda(M, \epsilon):=\prod_{i=1}^{\ell}\left(-\log \left|z_{i}\right|\right)^{M} \sum_{i=1}^{\ell}\left|z_{i}\right|^{\epsilon} .
$$

Theorem 7.4. There exists a $C^{\infty}$-map $\Phi: \mathcal{E}_{0}^{\triangle} \rightarrow \mathcal{E}^{\triangle}$ such that the following holds for some $\epsilon>0$ and $M>0$ with respect to $h_{0}$ and $g_{\mathbb{P}^{1}}$ :

$$
\begin{gathered}
\Phi^{*} \mathcal{S}-\mathcal{S}_{0}=O(\Lambda(M, \epsilon)), \quad \bar{\partial}_{\mathbb{P}^{1}, \mathcal{E}_{0}}\left(\Phi^{*} \mathcal{S}-\mathcal{S}_{0}\right)=O(\Lambda(M, \epsilon)), \\
\Phi^{*} \nabla_{\lambda}-\nabla_{\lambda, 0}=O(\Lambda(M, \epsilon)) .
\end{gathered}
$$

Note that $\Phi^{*} h$ and $h_{0}$ are mutually bounded up to log order, which follows from the weak norm estimate for acceptable bundles. (See Lemma 7.19 below.) Hence, we obtain the following estimate for some $M^{\prime}>0$ and $\epsilon^{\prime}>0$ by using Lemma 2.8

$$
\left|\Phi^{*} \mathcal{Q}-\mathcal{Q}_{0}\right|_{h_{0}}=O\left(\Lambda\left(M^{\prime}, \epsilon^{\prime}\right)\right) .
$$

In the one-dimensional case, the estimates in the two propositions are not so different. We also remark that $\Phi_{\sigma}$ in Theorem 7.3 also satisfies the estimates (7.23).

7.4.3. Proof of Theorem 7.3 , For the proof, we have only to consider the case that $\sigma$ is the identity. We use the symbol $Z(C)$ instead of $Z($ id, $C)$. Instead of considering $X^{*}(R)$, we will shrink $X$ around the origin. 
Decomposition. For any subset $I \subset \underline{\ell}$, let $m(I)$ be determined by the condition $m(I):=\min \{m \in I \mid m+1 \notin I\}$, in other words, $\{1, \ldots, m(I)\} \subset I$ but $m(I)+1$ $\notin I$. Let $q_{I}: \mathcal{P} \operatorname{ar}\left(\mathcal{P}_{0} \mathcal{E}^{0}, \underline{\ell}\right) \rightarrow \mathcal{P} a r\left(\mathcal{P}_{0} \mathcal{E}^{0}, I\right)$ and $r_{m(I)}: \mathbb{Z}^{\ell} \rightarrow \mathbb{Z}^{m(I)}$ be the natural projections. Let $\lambda_{0} \in \mathbb{C}_{\lambda}$. Let $\mathcal{K}$ denote a small neighbourhood of $\lambda_{0}$ in $\mathbb{C}_{\lambda}$. We set $\mathcal{X}:=\mathcal{K} \times X$. We use the symbols $\mathcal{D}_{i}, \mathcal{D}_{I}, \mathcal{D}$, etc., in similar meanings.

We have the induced filtrations ${ }^{i} F(i \in I)$ of $\mathcal{Q}_{0} \mathcal{E}_{\mid \mathcal{D}_{I}}$. For any $i \in I$, we have the residue endomorphisms $\operatorname{Res}_{i}(\mathbb{D})$ on ${ }^{I} \operatorname{Gr}_{\boldsymbol{b}}\left(\mathcal{Q}_{0} \mathcal{E}_{\mid \mathcal{D}_{I}}\right)$, which have the unique eigenvalues $-b_{i} \cdot \lambda$. Hence, the nilpotent part $\mathcal{N}_{i}$ is well defined. For $i \leq m(I)$, we set $\mathcal{N}(\underline{i}):=\sum_{j \leq i} \mathcal{N}_{j}$. Recall that the conjugacy classes of $\mathcal{N}(\underline{i})_{\mid(\lambda, P)}$ are independent of $(\lambda, P) \in \mathcal{D}_{I}$ (Lemma 12.47 of [22]). By considering the weight filtration of $\mathcal{N}(\underline{i})$, we obtain the filtration $W(\underline{i})$ of ${ }^{I} \operatorname{Gr}_{\boldsymbol{b}}\left(\mathcal{Q}_{0} \mathcal{E}_{\mid \mathcal{D}_{I}}\right)$ indexed by $\mathbb{Z}$ in the category of vector bundles on $\mathcal{D}_{I}$.

Lemma 7.8. We have a decomposition

$$
\mathcal{Q}_{0} \mathcal{E}_{\mid \mathcal{X}}=\bigoplus_{\substack{\boldsymbol{a} \in \mathcal{P} \text { ar }\left(\mathcal{P}_{0} \mathcal{E}^{0}, \underline{\ell}\right) \\ \boldsymbol{k} \in \mathbb{Z}^{\ell}}} U_{\boldsymbol{a}, \boldsymbol{k}}
$$

with the following property:

- For any subset $I \subset \underline{\ell}, \boldsymbol{b} \in \mathcal{P} a r\left(\mathcal{P}_{0} \mathcal{E}^{0}, I\right)$ and $\boldsymbol{h} \in \mathbb{Z}^{m(I)}$, put

$$
{ }^{I} U_{\boldsymbol{b}, \boldsymbol{h}}=\bigoplus_{\substack{\boldsymbol{a} \in q_{I}^{-1}(\boldsymbol{b}) \\ \boldsymbol{k} \in r_{m(I)}^{-1}(\boldsymbol{h})}} U_{\boldsymbol{a}, \boldsymbol{k}} \quad \text { and } \quad{ }^{I} U_{\boldsymbol{b}}=\bigoplus_{\boldsymbol{h} \in \mathbb{Z}^{m(I)}}{ }^{I} U_{\boldsymbol{b}, \boldsymbol{h}}
$$

Then, for any $c \in \mathbb{R}^{I}$,

$$
\bigoplus_{\boldsymbol{b} \leq \boldsymbol{c}}^{I} U_{\boldsymbol{b} \mid \mathcal{D}_{I}}=\bigcap_{i \in I}{ }^{i} F_{c_{i}}\left(\mathcal{Q}_{0} \mathcal{E}_{\mid \mathcal{D}_{I}}\right)
$$

Moreover, the following holds for any $\boldsymbol{n} \in \mathbb{Z}^{m(I)}$ under the identification ${ }^{I} U_{\boldsymbol{b} \mid \mathcal{D}_{I}}$ $\simeq{ }^{I} \operatorname{Gr}_{\boldsymbol{b}}\left(\mathcal{Q}_{0} \mathcal{E}\right)$ induced by 7.25 :

$$
\bigoplus_{\boldsymbol{h} \leq \boldsymbol{n}}{ }^{I} U_{\boldsymbol{b}, \boldsymbol{h} \mid \mathcal{D}_{I}}=\bigcap_{1 \leq i \leq m(I)} W_{n_{i}}(\underline{i})\left({ }^{I} \operatorname{Gr}_{\boldsymbol{b}}\left(\mathcal{Q}_{0} \mathcal{E}_{\mid \mathcal{D}_{I}}\right)\right) .
$$

Proof. Although this is essentially Corollary 4.47 of [22], we recall an outline for later use. The theorems and the definitions referred to in this proof are from 22 . By Theorem 12.43, the tuple $\left({ }^{i} F, \mathcal{N}(j) \mid i, j \in \underline{\ell}\right)$ is sequentially compatible in the sense of Definition 4.43. Hence, $\left({ }^{i} F, W(\underline{j}) \mid i, j \in \underline{\ell}\right)$ is compatible in the sense of Definition 4.39, as remarked in Lemma 4.44. By Proposition 4.41, there exists a splitting of $\left({ }^{i} F, W(\underline{j}) \mid i, j \in \underline{\ell}\right)$ in the sense of Definition 4.40. By applying 
Lemma 2.16, we can take a frame compatible with splittings. It is easy to take a decomposition as in 7.24 by using such a compatible frame.

Let $\left(\mathcal{Q} \mathcal{E}_{\boldsymbol{a}}, \mathbb{D}\right)$ be the prolongment of $\left(E_{\boldsymbol{a}}, \bar{\partial}_{\boldsymbol{a}}, \theta_{\boldsymbol{a}}, h_{\boldsymbol{a}}\right)$. Similarly, we have a decomposition

$$
\mathcal{Q}_{0} \mathcal{E}_{\boldsymbol{a} \mid \mathcal{X}}=\bigoplus_{\boldsymbol{k} \in \mathbb{Z}^{\ell}} U_{0, \boldsymbol{a}, \boldsymbol{k}}
$$

satisfying a similar condition. By our construction of $\left(\mathcal{E}_{0}^{\triangle}, \widetilde{\mathbb{D}}_{0}^{\triangle}, \mathcal{S}_{0}\right)$, we are given an isomorphism, for each $\boldsymbol{a} \in \mathcal{P} \operatorname{Par}\left(\mathcal{P}_{0} \mathcal{E}^{0}, \underline{\ell}\right)$,

$$
\nu_{\boldsymbol{a}}:{ }^{\ell} \operatorname{Gr}_{\boldsymbol{a}}\left(\mathcal{Q}_{0} \mathcal{E}\right) \simeq \mathcal{Q}_{0} \mathcal{E}_{\boldsymbol{a} \mid \mathcal{D}_{\underline{\underline{\ell}}}}
$$

Lemma 7.9. We may assume that $\nu_{\boldsymbol{a}}$ is compatible with the decompositions $\bigoplus_{\boldsymbol{k}} U_{0, \boldsymbol{a}, \boldsymbol{k} \mid \mathcal{D}_{\underline{\ell}}}$ and $\bigoplus_{\boldsymbol{k}} U_{\boldsymbol{a}, \boldsymbol{k} \mid \mathcal{D}_{\underline{\ell}}}$.

Proof. In Proposition 4.41 of [22], the construction of a splitting is given in a descending inductive way, and we can take any splitting of $\underline{\ell}^{-G_{a}}(\mathcal{Q E})$ of the filtrations $W(j)(j=1, \ldots, \ell)$ at the beginning. Thus, we obtain the conclusion.

Let $\nu_{\boldsymbol{a}, \boldsymbol{k}}$ denote the induced map $U_{0, \boldsymbol{a}, \boldsymbol{k} \mid \mathcal{D}_{\underline{\ell}}} \simeq U_{\boldsymbol{a}, \boldsymbol{k} \mid \mathcal{D}_{\underline{\ell}}}$.

Norm estimate. We recall the norm estimate for tame harmonic bundles. We take a $C^{\infty}$-frame $h_{\boldsymbol{a}, \boldsymbol{k}}^{\prime}$ of $U_{\boldsymbol{a}, \boldsymbol{k}}$ in 7.24 . We set

$$
\begin{aligned}
h_{\boldsymbol{a}, \boldsymbol{k}}^{(1)} & :=h_{\boldsymbol{a}, \boldsymbol{k}}^{\prime} \prod_{j=1}^{\ell}\left|z_{j}\right|^{-2 a_{j}}\left(-\log \left|z_{j}\right|\right)^{k_{j}-k_{j-1}} \\
& =h_{\boldsymbol{a}, \boldsymbol{k}}^{\prime} \prod_{p=1}^{\ell}\left|z_{p}\right|^{-2 a_{p}} \prod_{j=1}^{\ell-1}\left(\frac{-\log \left|z_{j}\right|}{-\log \left|z_{j+1}\right|}\right)^{k_{j}}\left(-\log \left|z_{\ell}\right|\right)^{k_{\ell}}
\end{aligned}
$$

(We formally set $k_{0}:=0$.) We obtain a $C^{\infty}$-hermitian metric $h^{(1)}=\bigoplus h_{\boldsymbol{a}, \boldsymbol{k}}^{(1)}$ on $\mathcal{Q E} \mathcal{E}_{\mid \mathcal{X}-\mathcal{D}}$. Theorem 13.25 of [22] implies the following lemma.

Lemma 7.10. $h$ and $h^{(1)}$ are mutually bounded on $\mathcal{K} \times Z(C)$.

\section{An estimate}

Lemma 7.11. Let $f$ be a holomorphic endomorphism of $\mathcal{Q}_{0} \mathcal{E}_{0}$ satisfying the following conditions:

- It preserves the filtrations ${ }^{i} F(i=1, \ldots, \ell)$. 
- For each $\boldsymbol{b} \in \mathbb{R}^{I}$, the induced endomorphism ${ }^{I} \mathrm{Gr}_{\boldsymbol{b}}^{F}(f)$ of $\bigoplus_{q_{I}(\boldsymbol{a})=\boldsymbol{b}} \mathcal{Q}_{0} \mathcal{E}_{\boldsymbol{a} \mid \mathcal{D}_{I}}$ preserves the weight filtrations $W(\underline{j})(j=1, \ldots, m(I))$.

- For each $\boldsymbol{a} \in \mathbb{R}^{\ell}$, the induced endomorphism $\underline{\ell}_{\mathrm{Gr}}^{F}(f)$ of $\mathcal{Q}_{0} \mathcal{E}_{\boldsymbol{a} \mid \mathcal{D}_{\underline{\ell}}}$ is 0 .

Then $|f|_{h_{0}}=O\left(\Lambda_{0}(\epsilon)\right)$ for some $\epsilon>0$ on $\mathcal{K} \times Z(C)$.

Proof. We take decompositions 7.26 for any $\boldsymbol{a}$. Applying Lemma 7.10 to $\left(E_{\boldsymbol{a}}, \bar{\partial}_{\boldsymbol{a}}\right.$, $\left.\theta_{\boldsymbol{a}}, h_{\boldsymbol{a}}\right)$ with the decomposition 7.26 , we take a $C^{\infty}$-hermitian metric $h_{0, \boldsymbol{a}}^{(1)}=$ $\bigoplus h_{0, \boldsymbol{a}, \boldsymbol{k}}^{(1)}$ on $\mathcal{Q}_{0} \mathcal{E}_{\boldsymbol{a} \mid \mathcal{X}-\mathcal{D}}$ and $h_{0}^{(1)}:=\bigoplus h_{0, \boldsymbol{a}}^{(1)}$ on $\mathcal{Q}_{0} \mathcal{E}_{0 \mid \mathcal{X}-\mathcal{D}}$ as above. We have the decomposition

$$
f=\sum f_{(\boldsymbol{a}, \boldsymbol{k}),\left(\boldsymbol{a}^{\prime}, \boldsymbol{k}^{\prime}\right)}, \quad f_{(\boldsymbol{a}, \boldsymbol{k}),\left(\boldsymbol{a}^{\prime}, \boldsymbol{k}^{\prime}\right)} \in \operatorname{Hom}\left(U_{0, \boldsymbol{a}^{\prime}, \boldsymbol{k}^{\prime}}, U_{0, \boldsymbol{a}, \boldsymbol{k}}\right) .
$$

We have only to show

$$
\left|f_{(\boldsymbol{a}, \boldsymbol{k}),\left(\boldsymbol{a}^{\prime}, \boldsymbol{k}^{\prime}\right)}\right|_{h_{0}^{(1)}}=O\left(\Lambda_{0}(\epsilon)\right)
$$

for any $(\boldsymbol{a}, \boldsymbol{k})$ and $\left(\boldsymbol{a}^{\prime}, \boldsymbol{k}^{\prime}\right)$ on $\mathcal{K} \times Z(C)$. Note that the induced metrics on $\operatorname{Hom}\left(U_{0, \boldsymbol{a}^{\prime}, \boldsymbol{k}^{\prime}}, U_{0, \boldsymbol{a}, \boldsymbol{k}}\right)_{\mid \mathcal{X}-\mathcal{D}}$ are of the form

$$
g_{(\boldsymbol{a}, \boldsymbol{k}),\left(\boldsymbol{a}^{\prime}, \boldsymbol{k}^{\prime}\right)} \prod_{j=1}^{\ell}\left|z_{j}\right|^{2\left(-a_{j}+a_{j}^{\prime}\right)} \prod_{j=1}^{\ell-1}\left(\frac{-\log \left|z_{j}\right|}{-\log \left|z_{j+1}\right|}\right)^{k_{j}-k_{j}^{\prime}}\left(-\log \left|z_{\ell}\right|\right)^{k_{\ell}-k_{\ell}^{\prime}},
$$

where $g_{(\boldsymbol{a}, \boldsymbol{k}),\left(\boldsymbol{a}^{\prime}, \boldsymbol{k}^{\prime}\right)}$ are $C^{\infty}$-metrics on $\operatorname{Hom}\left(U_{0, \boldsymbol{a}^{\prime}, \boldsymbol{k}^{\prime}}, U_{0, \boldsymbol{a}, \boldsymbol{k}}\right)$ over $\mathcal{X}$.

(I) Let us consider the case $\boldsymbol{a} \neq \boldsymbol{a}^{\prime}$. We define

$$
I_{+}:=\left\{i \mid a_{i}>a_{i}^{\prime}\right\}, \quad I_{-}:=\left\{i \mid a_{i}<a_{i}^{\prime}\right\}, \quad I_{0}:=\left\{i \mid a_{i}=a_{i}^{\prime}\right\} .
$$

Let $m$ be the number determined by $\{1, \ldots, m\} \subset I_{0}$ and $m+1 \notin I_{0}$. Since the parabolic filtrations are preserved, we have $f_{(\boldsymbol{a}, \boldsymbol{k}),\left(\boldsymbol{a}^{\prime}, \boldsymbol{k}^{\prime}\right) \mid \mathcal{D}_{i}}=0$ for any $i \in I_{+}$. Hence, there exists a holomorphic section $f_{(\boldsymbol{a}, \boldsymbol{k}),\left(\boldsymbol{a}^{\prime}, \boldsymbol{k}^{\prime}\right)}^{\prime}$ of $\operatorname{Hom}\left(U_{0, \boldsymbol{a}^{\prime}, \boldsymbol{k}^{\prime}}, U_{0, \boldsymbol{a}, \boldsymbol{k}}\right)$ such that

$$
f_{(\boldsymbol{a}, \boldsymbol{k}),\left(\boldsymbol{a}^{\prime}, \boldsymbol{k}^{\prime}\right)}=f_{(\boldsymbol{a}, \boldsymbol{k}),\left(\boldsymbol{a}^{\prime}, \boldsymbol{k}^{\prime}\right)}^{\prime} \prod_{i \in I_{+}} z_{i} .
$$

We have the following inequality for some $\epsilon>0$ :

$$
\prod_{i \in I_{+}}\left|z_{i}\right|^{1-a_{i}+a_{i}^{\prime}} \prod_{i \in I_{-}}\left|z_{i}\right|^{-a_{i}+a_{i}^{\prime}} \leq \prod_{i \in I_{+} \cup I_{-}}\left|z_{i}\right|^{\epsilon} \leq\left|z_{m+1}\right|^{\epsilon} .
$$

Let us consider the set $S=\left\{p \leq m \mid k_{p}>k_{p}^{\prime}\right\}$. If $S$ is not empty, let $p$ be its minimum. Note that $k_{t} \leq k_{t}^{\prime}$ for any $t<p$ and $k_{p}>k_{p}^{\prime}$ by our choice. Since the weight filtrations $W(\underline{j})(j=1, \ldots, p)$ are preserved on $\underline{p} \operatorname{Gr}^{F}$, we have $f_{(\boldsymbol{a}, \boldsymbol{k}),\left(\boldsymbol{a}^{\prime}, \boldsymbol{k}^{\prime}\right) \mid \mathcal{D}_{\underline{p}}}^{\prime}=0$. Hence, there exist holomorphic sections $f_{t,(\boldsymbol{a}, \boldsymbol{k}),\left(\boldsymbol{a}^{\prime}, \boldsymbol{k}^{\prime}\right)}^{\prime \prime}$ 
$(t=1, \ldots, p)$ of $\operatorname{Hom}\left(U_{0, \boldsymbol{a}^{\prime}, \boldsymbol{k}^{\prime}}, U_{0, \boldsymbol{a}, \boldsymbol{k}}\right)$ such that

$$
f_{(\boldsymbol{a}, \boldsymbol{k}),\left(\boldsymbol{a}^{\prime}, \boldsymbol{k}^{\prime}\right)}^{\prime}=\sum_{t=1}^{p} z_{t} \cdot f_{t,(\boldsymbol{a}, \boldsymbol{k}),\left(\boldsymbol{a}^{\prime}, \boldsymbol{k}^{\prime}\right)}^{\prime \prime} .
$$

We remark that for any $t \leq p$,

$$
\begin{aligned}
& \left|z_{t}\right| \prod_{j=1}^{\ell-1}\left(\frac{-\log \left|z_{j}\right|}{-\log \left|z_{j+1}\right|}\right)^{k_{j}-k_{j}^{\prime}}\left(-\log \left|z_{\ell}\right|\right)^{k_{\ell}-k_{\ell}^{\prime}} \\
& \quad \leq\left|z_{t}\right| \prod_{j=1}^{t-1} C^{k_{j}-k_{j}^{\prime}} \prod_{j=t}^{\ell-1}\left(\frac{-\log \left|z_{j}\right|}{-\log \left|z_{j+1}\right|}\right)^{k_{j}-k_{j}^{\prime}}\left(-\log \left|z_{\ell}\right|\right)^{k_{\ell}-k_{\ell}^{\prime}}=O\left(\left|z_{t}\right|^{1 / 2}\right) .
\end{aligned}
$$

By using 7.28$)-7.32$, we obtain $\left|f_{(\boldsymbol{a}, \boldsymbol{k}),\left(\boldsymbol{a}^{\prime}, \boldsymbol{k}^{\prime}\right)}\right|_{h_{0}^{(1)}}=\sum_{t=1}^{p} O\left(\left|z_{t}\right|^{1 / 2}\right)=O\left(\Lambda_{0}(1 / 2)\right)$.

If $S$ is empty, we have $k_{j} \leq k_{j}^{\prime}$ for $j=1, \ldots, m$. Hence,

$$
\begin{aligned}
& \text { 7.33) } \quad\left|z_{m+1}\right|^{\epsilon} \prod_{j=1}^{\ell-1}\left(\frac{-\log \left|z_{j}\right|}{-\log \left|z_{j+1}\right|}\right)^{k_{j}-k_{j}^{\prime}}\left(-\log \left|z_{\ell}\right|\right)^{k_{\ell}-k_{\ell}^{\prime}} \\
& \leq\left|z_{m+1}\right|^{\epsilon} \prod_{j=1}^{m} C^{k_{j}-k_{j}^{\prime}} \prod_{j=m+1}^{\ell-1}\left(\frac{-\log \left|z_{j}\right|}{-\log \left|z_{j+1}\right|}\right)^{k_{j}-k_{j}^{\prime}}\left(-\log \left|z_{\ell}\right|\right)^{k_{\ell}-k_{\ell}^{\prime}}=O\left(\left|z_{m+1}\right|^{\epsilon / 2}\right) .
\end{aligned}
$$

By using (7.28)-7.30) and (7.33), we obtain (7.27).

(II) Let us consider the case $\boldsymbol{a}=\boldsymbol{a}^{\prime}$. By the third assumption on $f$, there exist holomorphic sections $f_{i, \boldsymbol{a},\left(\boldsymbol{k}, \boldsymbol{k}^{\prime}\right)}$ of $\operatorname{Hom}\left(U_{0, \boldsymbol{a}, \boldsymbol{k}^{\prime}}, U_{0, \boldsymbol{a}, \boldsymbol{k}}\right)$ such that

$$
f_{(\boldsymbol{a}, \boldsymbol{k}),\left(\boldsymbol{a}, \boldsymbol{k}^{\prime}\right)}=\sum_{i=1}^{\ell} z_{i} \cdot f_{i, \boldsymbol{a},\left(\boldsymbol{k}, \boldsymbol{k}^{\prime}\right)} .
$$

Let us consider the set $S=\left\{p \mid k_{p}>k_{p}^{\prime}\right\}$. If $S$ is not empty, let $p$ be its minimum. Note that $k_{t} \leq k_{t}^{\prime}$ for any $t<p$ and $k_{p}>k_{p}^{\prime}$ by our choice. Since the weight filtrations $W(j)(j=1, \ldots, p)$ are preserved on $\underline{p} \mathrm{Gr}^{F}$, we have $f_{i, \boldsymbol{a},\left(\boldsymbol{k}, \boldsymbol{k}^{\prime}\right) \mid \mathcal{D}_{p}}=0$. Hence, there exist holomorphic sections $f_{t, i, \boldsymbol{a},\left(\boldsymbol{k}, \boldsymbol{k}^{\prime}\right)}^{\prime}(t=1, \ldots, p)$ of $\operatorname{Hom}\left(\overline{U_{0, \boldsymbol{a}, \boldsymbol{k}^{\prime}}}, U_{0, \boldsymbol{a}, \boldsymbol{k}}\right)$ such that

$$
f_{i, \boldsymbol{a},\left(\boldsymbol{k}, \boldsymbol{k}^{\prime}\right)}=\sum_{t=1}^{p} z_{t} \cdot f_{t, i, \boldsymbol{a},\left(\boldsymbol{k}, \boldsymbol{k}^{\prime}\right)}^{\prime}
$$

By using 7.32 and 7.35 , we obtain $\left|f_{i, \boldsymbol{a},\left(\boldsymbol{k}, \boldsymbol{k}^{\prime}\right)}\right|_{h_{0}^{(1)}}=O\left(\Lambda_{0}(1 / 2)\right)$.

If $S$ is empty, we have $k_{j} \leq k_{j}^{\prime}$ for $j=1, \ldots, \ell$. Hence,

$$
\prod_{j=1}^{\ell-1}\left(\frac{-\log \left|z_{j}\right|}{-\log \left|z_{j+1}\right|}\right)^{k_{j}-k_{j}^{\prime}}\left(-\log \left|z_{\ell}\right|\right)^{k_{\ell}-k_{\ell}^{\prime}}=O(1) .
$$


Consequently, $\left|f_{i, \boldsymbol{a},\left(\boldsymbol{k}, \boldsymbol{k}^{\prime}\right)}\right|_{h_{0}^{(1)}}=O(1)$. By using 7.34 , we obtain 7.27 . Thus, the lemma follows.

\section{Local isomorphism with a nice property}

Lemma 7.12. There exists a holomorphic isomorphism

$$
\Phi_{\mathcal{K}}: \mathcal{Q}_{0} \mathcal{E}_{0 \mid \mathcal{X}} \rightarrow \mathcal{Q}_{0} \mathcal{E}_{\mid \mathcal{X}}
$$

with the following properties:

- It preserves the filtrations ${ }^{i} F(i=1, \ldots, \ell)$.

- For each $\boldsymbol{b} \in \mathbb{R}^{I}$, the induced map $\bigoplus_{q_{I}(\boldsymbol{a})=\boldsymbol{b}} \mathcal{Q}_{0} \mathcal{E}_{\boldsymbol{a} \mid \mathcal{D}_{I}} \rightarrow{ }^{I} \operatorname{Gr}_{\boldsymbol{b}}^{F}\left(\mathcal{Q}_{0} \mathcal{E}_{\mid \mathcal{D}_{I}}\right)$ preserves the weight filtrations $W(\underline{j})(j=1, \ldots, m(I))$.

- For each $\boldsymbol{a} \in \mathbb{R}^{\ell}$, the induced map $\mathcal{Q}_{0} \mathcal{E}_{\boldsymbol{a} \mid \mathcal{D}_{\underline{\ell}}} \rightarrow{ }^{\ell} \operatorname{Gr}_{\boldsymbol{a}}^{F}\left(\mathcal{Q}_{0} \mathcal{E}_{\mid \mathcal{D}_{\underline{\ell}}}\right)$ is equal to $\nu_{\boldsymbol{a}}$.

Proof. We take decompositions (7.24) and (7.26) as in Lemma 7.9. We take an isomorphism $\widetilde{\nu}_{\boldsymbol{a}, \boldsymbol{k}}: U_{0, \boldsymbol{a}, \boldsymbol{k}} \simeq U_{\boldsymbol{a}, \boldsymbol{k}}$ such that $\widetilde{\nu}_{\boldsymbol{a}, \boldsymbol{k} \mid \mathcal{D}_{\ell}}=\nu_{\boldsymbol{a}, \boldsymbol{k}}$. We set $\Phi_{\mathcal{K}}:=\sum \widetilde{\nu}_{\boldsymbol{a}, \boldsymbol{k}}$. It is easy to check that $\Phi_{\mathcal{K}}$ has the desired property.

By the norm estimate (Lemma $7.10, \Phi_{\mathcal{K}}$ and $\Phi_{\mathcal{K}}^{-1}$ are bounded on $\mathcal{K} \times Z(C)$.

Lemma 7.13. We have the following estimate for some $\epsilon>0$ with respect to $h_{0}$ and $g_{\mathbb{P}^{1}}$ on $\mathcal{K} \times Z(C)$ :

$$
\Phi_{\mathcal{K}}^{*} \nabla_{\lambda}-\nabla_{\lambda, 0}=O\left(\Lambda_{0}(\epsilon)\right) .
$$

Proof. We put $F:=\Phi_{\mathcal{K}}^{*} \nabla_{\lambda}\left(\lambda^{2} \partial_{\lambda}\right)-\nabla_{\lambda, 0}\left(\lambda^{2} \partial_{\lambda}\right)$. It is easy to observe that $F$ satisfies the conditions in Lemma 7.11. Hence, the lemma follows from Lemma 7.11 .

Let $\Phi_{\mathcal{K}}$ and $\Phi_{\mathcal{K}}^{\prime}$ be morphisms as in Lemma 7.12 . We set $G:=\Phi_{\mathcal{K}}^{-1} \circ \Phi_{\mathcal{K}}^{\prime}$.

Lemma 7.14. We have the following estimates for some $\epsilon>0$ on $\mathcal{K} \times Z(C)$ :

$$
|G-\mathrm{id}|_{h_{0}}=O\left(\Lambda_{0}(\epsilon)\right), \quad\left|\nabla_{\lambda, 0}\left(\lambda^{2} \partial_{\lambda}\right) G\right|_{h_{0}}=O\left(\Lambda_{0}(\epsilon)\right) .
$$

Proof. We have only to apply Lemma 7.11 to $G-$ id and $\nabla_{\lambda, 0}\left(\lambda^{2} \partial_{\lambda}\right) G$.

Let $\sigma: \mathbb{C}_{\lambda} \rightarrow \mathbb{C}_{\mu}$ given by $\sigma(\lambda)=-\bar{\lambda}$. The induced map $\mathbb{C}_{\lambda} \times X \rightarrow \mathbb{C}_{\mu} \times X^{\dagger}$ is also denoted by $\sigma$.

Lemma 7.15. We can take a holomorphic isomorphism

$$
\Phi_{\sigma(\mathcal{K})}^{\dagger}: \mathcal{Q}_{<\delta} \mathcal{E}_{0 \mid \sigma(\mathcal{X})}^{\dagger} \rightarrow \mathcal{Q}_{<\delta} \mathcal{E}_{\mid \sigma(\mathcal{X})}^{\dagger}
$$

satisfying the conditions: (i) it preserves the filtrations ${ }^{i} F(i=1, \ldots, \ell)$, (ii) the induced morphisms on ${ }^{I} \mathrm{Gr}_{-\boldsymbol{a}}^{F}$ preserve the weight filtrations $W(j)(j=1, \ldots, m(I))$, (iii) the induced morphism on $\stackrel{\ell}{G r}_{-a}^{F}$ is equal to the given one. 
Proof. This can be shown by the argument in the proof of Lemma 7.12. More directly, we have the isomorphisms $\mathcal{Q}_{<\delta} \mathcal{E}_{0 \mid \sigma(\mathcal{X})}^{\dagger} \simeq \sigma^{*}\left(\mathcal{Q}_{0} \mathcal{E}_{0 \mid \mathcal{X}}\right)^{\vee}$ and $\mathcal{Q}_{<\delta} \mathcal{E}_{\mid \sigma(\mathcal{X})}^{\dagger} \simeq$ $\sigma^{*}\left(\mathcal{Q}_{0} \mathcal{E}_{\mid \mathcal{X}}\right)^{\vee}$, and $\sigma^{*}\left(\Phi_{\mathcal{K}}\right)^{\vee}$ satisfies the conditions.

Lemma 7.16. Let $\Phi_{\mathcal{K}}$ and $\Phi_{\sigma(\mathcal{K})}^{\dagger}$ satisfy the above conditions. Set

$$
H:=\mathcal{S}_{0}-\mathcal{S}\left(\Phi_{\mathcal{K}} \otimes \sigma^{*} \Phi_{\sigma(\mathcal{K})}^{\dagger}\right): \mathcal{Q}_{0} \mathcal{E}_{0 \mid \mathcal{X}} \otimes \sigma^{*}\left(\mathcal{Q}_{<\delta} \mathcal{E}_{0 \mid \sigma(\mathcal{X})}^{\dagger}\right) \rightarrow \mathcal{O}_{\mathcal{X}}
$$

Then $H=O\left(\Lambda_{0}(\epsilon)\right)$ with respect to $h_{0}$ for some $\epsilon>0$ on $\mathcal{K} \times Z(C)$.

Proof. If $\Phi_{\sigma(\mathcal{K})}^{\dagger}$ is given by $\sigma^{*} \Phi_{\mathcal{K}}^{\vee}$, we have $H=0$. Hence, we have only to show that the property is independent of the choice of $\Phi_{\sigma(\mathcal{K})}^{\dagger}$.

Let $\Phi_{i, \sigma(\mathcal{K})}^{\dagger}(i=1,2)$ be as in Lemma 7.15. Note that $h$ and $h_{0}$ are mutually bounded through $\Phi_{1, \sigma(\mathcal{K})}^{\dagger}$ on $\sigma(\mathcal{K}) \times Z(C)$. By using Lemma 7.14 we obtain $\Phi_{1, \sigma(\mathcal{K})}^{\dagger}-\Phi_{2, \sigma(\mathcal{K})}^{\dagger}=O\left(\Lambda_{0}(\epsilon)\right)$ for some $\epsilon>0$ with respect to $h$ and $h_{0}$. It follows that $\mathcal{S} \circ\left(\Phi_{\mathcal{K}} \otimes \sigma^{*}\left(\Phi_{1, \sigma(\mathcal{K})}^{\dagger}-\Phi_{2, \sigma(\mathcal{K})}^{\dagger}\right)\right)=O\left(\Lambda_{0}(\epsilon)\right)$ with respect to $h_{0}$. Thus, the proof is finished.

Local $C^{\infty}$-isomorphisms. Let $\Phi_{\mathcal{K}}^{p}(p=0, \ldots, m)$ be as in Lemma 7.12 and let $a_{p}(p=0, \ldots, m)$ be non-negative $C^{\infty}$-functions on $\mathcal{K}$ such that $\sum a_{p}=1$. We set $\Phi_{\mathcal{K}}:=\sum_{p=0}^{m} a_{p} \Phi_{\mathcal{K}}^{p}$. We also set $G:=\left(\Phi_{\mathcal{K}}^{0}\right)^{-1} \circ \Phi_{\mathcal{K}}$ and $G^{p}:=\left(\Phi_{\mathcal{K}}^{0}\right)^{-1} \circ \Phi_{\mathcal{K}}^{p}$. By Lemma 7.14 $\left|G^{p}-\mathrm{id}\right|_{h_{0}}=O\left(\Lambda_{0}(\epsilon)\right)$, and hence $|G-\mathrm{id}|_{h_{0}}=O\left(\Lambda_{0}(\epsilon)\right)$ for some $\epsilon>0$ on $\mathcal{K} \times Z(C)$.

Lemma 7.17. The following estimate holds for some $\epsilon>0$ with respect to $h_{0}$ on $\mathcal{K} \times Z(C)$ :

$$
\Phi_{\mathcal{K}}^{-1} \circ \nabla_{\lambda}\left(\lambda^{2} \partial_{\lambda}\right) \circ \Phi_{\mathcal{K}}-\nabla_{\lambda, 0}\left(\lambda^{2} \partial_{\lambda}\right)=O\left(\Lambda_{0}(\epsilon)\right)
$$

Proof. We have the following equalities:

$$
\begin{aligned}
& \Phi_{\mathcal{K}}^{-1} \circ \nabla_{\lambda, 0}\left(\partial_{\lambda}\right) \circ \Phi_{\mathcal{K}}-\nabla_{\lambda, 0}\left(\partial_{\lambda}\right) \\
& \quad=\left(\Phi_{\mathcal{K}}^{-1} \circ \Phi_{\mathcal{K}}^{0}\right) \circ\left(\Phi_{\mathcal{K}}^{0}\right)^{-1} \circ \nabla_{\lambda}\left(\partial_{\lambda}\right) \circ\left(\Phi_{\mathcal{K}}^{0}\right) \circ\left(\left(\Phi_{\mathcal{K}}^{0}\right)^{-1} \circ \Phi_{\mathcal{K}}\right)-\nabla_{\lambda, 0}\left(\partial_{\lambda}\right) \\
& \quad=G^{-1} \circ\left(\left(\Phi_{\mathcal{K}}^{0}\right)^{*} \nabla_{\lambda}\left(\partial_{\lambda}\right)-\nabla_{\lambda, 0}\left(\partial_{\lambda}\right)\right) \circ G+G^{-1} \nabla_{\lambda, 0}\left(\partial_{\lambda}\right) G .
\end{aligned}
$$

By Lemma 7.13, $\left(\Phi_{\mathcal{K}}^{0}\right)^{*} \nabla_{\lambda}\left(\lambda^{2} \partial_{\lambda}\right)-\nabla_{\lambda, 0}\left(\lambda^{2} \partial_{\lambda}\right)=O\left(\Lambda_{0}(\epsilon)\right)$. We also have

$$
\nabla_{\lambda, 0}\left(\lambda^{2} \partial_{\lambda}\right) G=\sum \lambda^{2} \frac{\partial a_{p}}{\partial \lambda}\left(G^{p}-\mathrm{id}\right)=O\left(\Lambda_{0}(\epsilon)\right)
$$

Thus, we obtain the lemma.

Let $\Phi_{\sigma(\mathcal{K})}^{\dagger q}\left(q=0,1, \ldots, m^{\prime}\right)$ be as in Lemma 7.15, and let $b_{q}$ be non-negative $C^{\infty}$-functions on $\sigma(\mathcal{K})$ such that $\sum b_{q}=1$. Put $\Phi_{\sigma(\mathcal{K})}^{\dagger}:=\sum b_{q} \Phi_{\sigma(S)}^{\dagger q}$. 
Lemma 7.18. Set $H:=\mathcal{S}\left(\Phi_{\mathcal{K}} \otimes \sigma^{*}\left(\Phi_{\sigma(\mathcal{K})}^{\dagger}\right)\right)-\mathcal{S}_{0}$. Then we have the following estimates on $\mathcal{K} \times Z(C)$ with respect to $h_{0}$ for some $\epsilon>0$ :

$$
H=O\left(\Lambda_{0}(\epsilon)\right), \quad \bar{\partial}_{\mathcal{E}_{0}^{\triangle}, \mathbb{P}^{1}} H=O\left(\Lambda_{0}(\epsilon)\right) .
$$

Proof. This follows from Lemma 7.16 .

Construction of an approximating map. We take $0<R_{1}<R_{2}<1$. We set $\mathcal{K}_{1}:=\left\{\lambda|| \lambda \mid \leq R_{2}\right\}$ and $\mathcal{K}_{2}:=\left\{\lambda\left|R_{1} \leq\right| \lambda \mid \leq R_{1}^{-1}\right\}$. We take a partition of unity $\left(\chi_{\mathcal{K}_{1}}, \chi_{\mathcal{K}_{2}}, \chi_{\sigma\left(\mathcal{K}_{1}\right)}\right)$ on $\mathbb{P}^{1}$ subordinated to $\left\{\mathcal{K}_{1}, \mathcal{K}_{2}, \sigma\left(\mathcal{K}_{1}\right)\right\}$.

We take a holomorphic isomorphism $\Phi_{\mathcal{K}_{1}}: \mathcal{Q}_{0} \mathcal{E}_{0 \mid \mathcal{K}_{1} \times X} \rightarrow \mathcal{Q}_{0} \mathcal{E}_{\mid \mathcal{K}_{1} \times X}$ as in Lemma 7.12. Similarly, we take a holomorphic isomorphism $\Phi_{\sigma\left(\mathcal{K}_{1}\right)}^{\dagger}: \mathcal{Q}_{<\delta} \mathcal{E}_{0 \mid \sigma(\mathcal{K}) \times X^{\dagger}}^{\dagger}$ $\rightarrow \mathcal{Q}_{<\delta} \mathcal{E}_{\mid \sigma(\mathcal{K}) \times X^{\dagger}}^{\dagger}$ as in Lemma 7.15 .

We can take a flat isomorphism

$$
\Phi_{\mathcal{K}_{2}}:\left(\mathcal{E}_{0}, \widetilde{\mathbb{D}}_{0}^{f}\right)_{\mid \mathcal{K}_{2} \times(X-D)} \rightarrow\left(\mathcal{E}, \widetilde{\mathbb{D}}^{f}\right)_{\mid \mathcal{K}_{2} \times(X-D)} .
$$

We may assume that $\Phi_{\mathcal{K}_{2}}$ is extended to isomorphisms $\mathcal{Q}_{0} \mathcal{E}_{0 \mid \mathcal{K}_{2} \times X} \simeq \mathcal{Q}_{0} \mathcal{E}_{\mid \mathcal{K}_{2} \times X}$ and $\mathcal{Q}_{<\delta} \mathcal{E}_{0 \mid \mathcal{K}_{2} \times X^{\dagger}}^{\dagger} \simeq \mathcal{Q}_{<\delta} \mathcal{E}_{\mid \mathcal{K}_{2} \times X^{\dagger}}^{\dagger}$ with the property in Lemmas 7.12 and 7.15 . We set

$$
\Phi:=\chi_{\mathcal{K}_{1}} \Phi_{\mathcal{K}_{1}}+\chi_{\mathcal{K}_{2}} \Phi_{\mathcal{K}_{2}}+\chi_{\sigma\left(\mathcal{K}_{1}\right)} \Phi_{\sigma\left(\mathcal{K}_{1}\right)}^{\dagger}
$$

By using Lemmas 7.17 and 7.18 , we can check that $\Phi$ satisfies the estimates in 7.21. Thus, the proof of Theorem 7.3 is finished.

\subsubsection{Proof of Theorem 7.4}

Decomposition. We have a decomposition

$$
\mathcal{Q}_{0} \mathcal{E}_{\mathcal{X}}=\bigoplus_{\boldsymbol{a} \in \mathcal{P} a r\left(\mathcal{P}_{0} \mathcal{E}^{0}, \underline{\ell}\right)} U_{\boldsymbol{a}}
$$

with the following property:

- For any subset $I \subset \underline{\ell}$ and $\boldsymbol{b} \in \mathcal{P} a r\left(\mathcal{P}_{0} \mathcal{E}^{0}, I\right)$, put ${ }^{I} U_{\boldsymbol{b}}=\bigoplus_{\boldsymbol{a} \in q_{I}^{-1}(\boldsymbol{b})} U_{\boldsymbol{a}}$. Then, for any $c \in \mathbb{R}^{I}$,

$$
\bigoplus_{\boldsymbol{b} \leq \boldsymbol{c}}^{I} U_{\boldsymbol{b} \mid \mathcal{D}_{I}}=\bigcap_{i \in I}{ }^{i} F_{c_{i}}\left(\mathcal{Q}_{0} \mathcal{E}_{\mid \mathcal{D}_{I}}\right)
$$

Weak norm estimate. We take a $C^{\infty}$-frame $h_{\boldsymbol{a}}^{\prime}$ of $U_{\boldsymbol{a}}$ in 7.39 . We set $h_{\boldsymbol{a}}^{(2)}:=$ $h_{\boldsymbol{a}}^{\prime} \prod_{j=1}^{\ell}\left|z_{j}\right|^{-2 a_{j}}$. We obtain a $C^{\infty}$-hermitian metric $h^{(2)}=\bigoplus h_{\boldsymbol{a}}^{(2)}$ on $\mathcal{Q} \mathcal{E}_{\mid \mathcal{X}-\mathcal{D}}$. Proposition 8.70 of [22] implies the following lemma. 
Lemma 7.19. $h$ and $h^{(2)}$ are mutually bounded up to log order, namely,

$$
h^{(2)} \cdot C^{-1}\left(\sum_{i=1}^{\ell}-\log \left|z_{i}\right|\right)^{-N} \leq h \leq h^{(2)} \cdot C\left(\sum_{i=1}^{\ell}-\log \left|z_{i}\right|\right)^{N}
$$

for some $C>0$ and $N>0$.

\section{An estimate}

Lemma 7.20. Let $f$ be a holomorphic endomorphism of $\mathcal{Q}_{0} \mathcal{E}_{0}$ satisfying the following conditions:

- It preserves the filtrations ${ }^{i} F(i=1, \ldots, \ell)$.

- For each $\boldsymbol{a} \in \mathbb{R}^{\ell}$, the induced endomorphism $\underline{\ell}_{\mathrm{Gr}_{\boldsymbol{a}}^{F}}^{F}(f)$ of $\mathcal{Q} \mathcal{E}_{\boldsymbol{a} \mid \mathcal{D}_{\underline{\ell}}}$ is 0 .

Then $|f|_{h_{0}}=O(\Lambda(M, \epsilon))$ for some $M>0$ and $\epsilon>0$.

Proof. We take a decomposition of $\mathcal{Q}_{0} \mathcal{E}_{0}$ like 7.39 . Applying the weak norm estimate to $\left(E_{\boldsymbol{a}}, \bar{\partial}_{\boldsymbol{a}}, \theta_{\boldsymbol{a}}, h_{\boldsymbol{a}}\right)$ with the decomposition $(7.26)$, we choose a $C^{\infty}$-hermitian metric $h_{\boldsymbol{a}}^{(2)}$ on $\mathcal{Q} \mathcal{E}_{\boldsymbol{a} \mid \mathcal{X}-\mathcal{D}}$, and $h_{0}^{(2)}=\bigoplus h_{\boldsymbol{a}}^{(2)}$ on $\mathcal{Q} \mathcal{E}_{0 \mid \mathcal{X}-\mathcal{D}}$. We have the decomposition

$$
f=\sum f_{\boldsymbol{a}, \boldsymbol{a}^{\prime}}, \quad f_{\boldsymbol{a}, \boldsymbol{a}^{\prime}} \in \operatorname{Hom}\left(U_{0, \boldsymbol{a}^{\prime}}, U_{0, \boldsymbol{a}}\right) .
$$

We have only to show $\left|f_{\boldsymbol{a}, \boldsymbol{a}^{\prime}}\right|_{h_{0}^{(2)}}=O(\Lambda(M, \epsilon))$ for any $\boldsymbol{a}$ and $\boldsymbol{a}^{\prime}$. Assume $\boldsymbol{a} \neq \boldsymbol{a}^{\prime}$. We define

$$
I_{+}:=\left\{i \mid a_{i}>a_{i}^{\prime}\right\}, \quad I_{-}:=\left\{i \mid a_{i}<a_{i}^{\prime}\right\}, \quad I_{0}:=\left\{i \mid a_{i}=a_{i}^{\prime}\right\} .
$$

Since the parabolic filtrations are preserved, we have $f_{\boldsymbol{a}, \boldsymbol{a}^{\prime} \mid \mathcal{D}_{i}}=0$ for any $i \in I_{+}$. Hence, there exists a holomorphic section $f_{\boldsymbol{a}, \boldsymbol{a}^{\prime}}^{\prime}$ such that $f_{\boldsymbol{a}, \boldsymbol{a}^{\prime}}=f_{\boldsymbol{a}, \boldsymbol{a}^{\prime}}^{\prime} \prod_{i \in I_{+}} z_{i}$. We have the inequality as in 7.30. Thus, we obtain the desired estimate for $f_{\boldsymbol{a}, \boldsymbol{a}^{\prime}}$ in the case $\boldsymbol{a} \neq \boldsymbol{a}^{\prime}$.

If $\boldsymbol{a}=\boldsymbol{a}^{\prime}$, then $f_{\boldsymbol{a}, \boldsymbol{a} \mid \mathcal{D}_{\underline{\ell}}}=0$. Hence, there are holomorphic sections $f_{t, \boldsymbol{a}}$ of $\operatorname{Hom}\left(\mathcal{Q}_{0} \mathcal{E}_{\boldsymbol{a}}, \mathcal{Q}_{0} \mathcal{E}_{\boldsymbol{a}}\right)$ such that $f_{\boldsymbol{a}, \boldsymbol{a}}=\sum z_{t} f_{t, \boldsymbol{a}}$. Because

$$
\left|f_{t, \boldsymbol{a}}\right|_{h_{0}}=O\left(\sum_{i=1}^{\ell}\left(-\log \left|z_{i}\right|\right)^{N}\right)
$$

we obtain the desired estimate.

Local isomorphism with a nice property. We can show the following lemma by the argument in the proof of Lemma 7.12 .

Lemma 7.21. There exists a holomorphic isomorphism

$$
\Phi_{\mathcal{K}}: \mathcal{Q} \mathcal{E}_{0 \mid \mathcal{X}} \rightarrow \mathcal{Q} \mathcal{E}_{\mid \mathcal{X}}
$$


such that (i) it preserves the filtrations ${ }^{i} F(i=1, \ldots, \ell)$, (ii) for each $\boldsymbol{a} \in \mathbb{R}^{\ell}$, the induced map $\mathcal{Q E}_{\boldsymbol{a} \mid \mathcal{D}_{\underline{\underline{\ell}}}} \rightarrow \stackrel{\ell}{\mathrm{Gr}_{\boldsymbol{a}}^{F}}\left(\mathcal{Q E}_{\mid \mathcal{D}_{\underline{\underline{\ell}}}}\right)$ is equal to $\nu_{\boldsymbol{a}}$.

Similarly, we can find a holomorphic isomorphism $\Phi_{\sigma(\mathcal{K})}^{\dagger}: \mathcal{Q}_{<\delta} \mathcal{E}_{0 \mid \sigma(\mathcal{X})}^{\dagger} \rightarrow$ $\mathcal{Q}_{<\delta} \mathcal{E}_{\mid \sigma(\mathcal{X})}^{\dagger}$ such that (i) it preserves the filtrations ${ }^{i} F(i=1, \ldots, \ell)$, (iii) the induced morphism on $\underline{\mathrm{Gr}}_{-\boldsymbol{a}}^{F}$ is equal to the given one.

By the weak norm estimate, $\Phi_{\mathcal{K}}$ and $\Phi_{\mathcal{K}}^{-1}$ are bounded up to log order. We can show the following lemma by using Lemma 7.20.

Lemma 7.22. We have $\Phi_{\mathcal{K}}^{*} \nabla_{\lambda}-\nabla_{\lambda, 0}=O(\Lambda(M, \epsilon))$ for some $\epsilon>0$ and $M>0$ with respect to $h_{0}$ and $g_{\mathbb{P}^{1}}$.

Let $\Phi_{\mathcal{K}}$ and $\Phi_{\mathcal{K}}^{\prime}$ be morphisms as in Lemma 7.21. We set $G:=\Phi_{\mathcal{K}}^{-1} \circ \Phi_{\mathcal{K}}^{\prime}$.

Lemma 7.23. We have the following estimates for some positive $\epsilon$ and $M$ :

$$
|G-\mathrm{id}|_{h_{0}}=O(\Lambda(M, \epsilon)), \quad\left|\nabla_{\lambda, 0}\left(\lambda^{2} \partial_{\lambda}\right) G\right|_{h_{0}}=O(\Lambda(M, \epsilon)) .
$$

Proof. This follows from Lemma 7.20 .

Lemma 7.24. Let $\Phi_{\mathcal{K}}$ and $\Phi_{\sigma(\mathcal{K})}^{\dagger}$ satisfy the above conditions. Set

$$
H:=\mathcal{S}_{0}-\mathcal{S}\left(\Phi_{\mathcal{K}} \otimes \sigma^{*} \Phi_{\sigma(\mathcal{K})}^{\dagger}\right): \mathcal{Q}_{0} \mathcal{E}_{0 \mid \mathcal{X}} \otimes \sigma^{*}\left(\mathcal{Q}_{<\delta} \mathcal{E}_{0 \mid \sigma(\mathcal{X})}^{\dagger}\right) \rightarrow \mathcal{O}_{\mathcal{X}} .
$$

Then $H=O(\Lambda(M, \epsilon))$ with respect to $h_{0}$ for some $\epsilon>0$ and $M>0$.

Proof. This can be shown by the argument in the proof of Lemma 7.16.

Local $C^{\infty}$-isomorphisms. Let $\Phi_{\mathcal{K}}^{p}(p=0, \ldots, m)$ be as in Lemma 7.21, and let $a_{p}(p=0, \ldots, m)$ be non-negative $C^{\infty}$-functions on $\mathcal{K}$ such that $\sum a_{p}=1$. We set $\Phi_{\mathcal{K}}:=\sum_{p=0}^{m} a_{p} \Phi_{\mathcal{K}}^{p}, G:=\left(\Phi_{\mathcal{K}}^{0}\right)^{-1} \circ \Phi_{\mathcal{K}}$ and $G^{p}:=\left(\Phi_{\mathcal{K}}^{0}\right)^{-1} \circ \Phi_{\mathcal{K}}^{p}$. By Lemma $7.23 .\left|G^{p}-\mathrm{id}\right|_{h_{0}}=O\left(\Lambda_{0}(\epsilon)\right)$, and hence $|G-\mathrm{id}|_{h_{0}}=O\left(\Lambda_{0}(\epsilon)\right)$ for some $\epsilon>0$ and $M>0$.

We can show the following estimate by using an argument in the proof of Lemma 7.17 together with Lemma 7.22 .

$$
\Phi_{\mathcal{K}}^{-1} \circ \nabla_{\lambda}\left(\lambda^{2} \partial_{\lambda}\right) \circ \Phi_{\mathcal{K}}-\nabla_{\lambda, 0}\left(\lambda^{2} \partial_{\lambda}\right)=O(\Lambda(M, \epsilon)) .
$$

Let $\Phi_{\sigma(\mathcal{K})}^{\dagger q}\left(q=0,1, \ldots, m^{\prime}\right)$ be as in Lemma 7.15, and let $b_{q}$ be non-negative $C^{\infty}$-functions on $\sigma(\mathcal{K})$ such that $\sum b_{q}=1$. We set $\Phi_{\sigma(\mathcal{K})}^{\dagger}:=\sum b_{q} \Phi_{\sigma(S)}^{\dagger q}$ and $H:=\mathcal{S}\left(\Phi_{\mathcal{K}} \otimes \sigma^{*}\left(\Phi_{\sigma(\mathcal{K})}^{\dagger}\right)\right)-\mathcal{S}_{0}$. Then we can show the following estimates with respect to $h_{0}$ and $g_{\mathbb{P}^{1}}$ for some positive $\epsilon$ and $M$, by using Lemma 7.24 .

$$
H=O(\Lambda(M, \epsilon)), \quad \bar{\partial}_{\mathcal{E}_{0}^{\triangle}, \mathbb{P}^{1}} H=O(\Lambda(M, \epsilon)) .
$$


Construction. We take $0<R_{1}<R_{2}<1$. We set $\mathcal{K}_{1}:=\left\{\lambda|| \lambda \mid \leq R_{2}\right\}$ and $\mathcal{K}_{2}:=\left\{\lambda\left|R_{1} \leq\right| \lambda \mid \leq R_{1}^{-1}\right\}$. We take a partition of unity $\left(\chi_{\mathcal{K}_{1}}, \chi_{\mathcal{K}_{2}}, \chi_{\sigma\left(\mathcal{K}_{1}\right)}\right)$ on $\mathbb{P}^{1}$ subordinated to $\left\{\mathcal{K}_{1}, \mathcal{K}_{2}, \sigma\left(\mathcal{K}_{1}\right)\right\}$.

We take holomorphic isomorphisms

$$
\Phi_{\mathcal{K}_{1}}: \mathcal{Q E}_{0 \mid \mathcal{K}_{1} \times X} \rightarrow \mathcal{Q E}_{\mid \mathcal{K}_{1} \times X}, \quad \Phi_{\sigma\left(\mathcal{K}_{1}\right)}^{\dagger}: \mathcal{Q}_{<\delta} \mathcal{E}_{0 \mid \sigma\left(\mathcal{K}_{1}\right) \times X^{\dagger}}^{\dagger} \rightarrow \mathcal{Q}_{<\delta} \mathcal{E}_{\mid \sigma\left(\mathcal{K}_{1}\right) \times X^{\dagger}}^{\dagger}
$$

as in Lemma 7.21. We can take a flat isomorphism

$$
\Phi_{\mathcal{K}_{2}}:\left(\mathcal{E}_{0}, \widetilde{\mathbb{D}}_{0}^{f}\right)_{\mid \mathcal{K}_{2} \times(X-D)} \rightarrow\left(\mathcal{E}, \widetilde{\mathbb{D}}^{f}\right)_{\mid \mathcal{K}_{2} \times(X-D)} .
$$

We set $\Phi:=\chi_{\mathcal{K}_{1}} \Phi_{\mathcal{K}_{1}}+\chi_{\mathcal{K}_{2}} \Phi_{\mathcal{K}_{2}}+\chi_{\sigma\left(\mathcal{K}_{1}\right)} \Phi_{\sigma\left(\mathcal{K}_{1}\right)}^{\dagger}$. By using 7.41) and 7.42 , we can check that $\Phi$ satisfies the estimates in 7.23$)$. Thus, the proof of Theorem 7.4 is finished.

\section{§8. An application to HS-orbits}

\section{§8.1. Preliminaries}

8.1.1. Compatibility of real structure and Stokes structure. Let $X$ be a complex manifold. We set $\mathcal{X}:=\mathbb{C}_{\lambda} \times X$ and $\mathcal{X}^{0}:=\{0\} \times X$. Let $\left(H, H_{\mathbb{R}}^{\prime}, \nabla\right)$ be a TER structure on $\mathcal{X}$. (TER structure means TERP structure minus a pairing.) We say that $H$ is unramifiedly pseudo-good if the following holds:

- We are given a good set of irregular values $\operatorname{Irr}(\nabla) \subset M\left(\mathcal{X}, \mathcal{X}^{0}\right) / H(\mathcal{X})$ on the level -1 . Namely, (i) every element $\mathfrak{a}$ of $\operatorname{Irr}(\nabla)$ is of the form $\mathfrak{a}=\lambda^{-1} \mathfrak{a}^{\prime}$ for some holomorphic function $\mathfrak{a}^{\prime}$ on $X$, (ii) $\mathfrak{a}^{\prime}-\mathfrak{b}^{\prime}$ is nowhere vanishing for distinct $\lambda^{-1} \mathfrak{a}^{\prime}, \lambda^{-1} \mathfrak{b}^{\prime} \in \operatorname{Irr}(\nabla)$.

- $H$ has the formal decomposition

$$
(H, \nabla)_{\mid \widehat{\mathcal{X}}^{0}}=\bigoplus_{\mathfrak{a} \in \operatorname{Irr}(\nabla)}\left(\widehat{H}_{\mathfrak{a}}, \widehat{\nabla}_{\mathfrak{a}}\right)
$$

such that $\widehat{\nabla}_{\mathfrak{a}}-d \mathfrak{a}$ is regular. Note that they are not assumed to be logarithmic.

(See also Subsection 5.1.3) If $X$ is a point, this means that $H$ requires no ramification in the sense of [12.

By a classical theory (see also Subsection 5.1.3), we have the Stokes filtration $\mathcal{F}^{S}$ indexed by $\left(\operatorname{Irr}(\nabla), \leq_{S}\right)$ for each small sector $S$ of $\mathcal{X}-\mathcal{X}^{0}$. We say that the real structure and the Stokes structure are compatible if the Stokes filtration on any small sector $S$ comes from a flat filtration of $H_{\mathbb{R} \mid S}^{\prime}$. (See [18].)

By taking Gr of $(H, \nabla)$ with respect to the Stokes filtrations, we obtain a $T E$-structure $\operatorname{Gr}_{\mathfrak{a}}(H, \nabla)$ for $\mathfrak{a} \in \operatorname{Irr}(\nabla)$. As observed in [12], if the real structure 
and the Stokes structure are compatible, $\operatorname{Gr}_{\mathfrak{a}}(H, \nabla)$ is enriched to a TER structure denoted by $\operatorname{Gr}_{\mathfrak{a}}\left(H, H_{\mathbb{R}}^{\prime}, \nabla\right)$. If $\left(H, H_{\mathbb{R}}^{\prime}, \nabla\right)$ is enriched to a TERP structure $\left(H, H_{\mathbb{R}}^{\prime}, \nabla, P, w\right), \operatorname{Gr}_{\mathfrak{a}}\left(H, H_{\mathbb{R}}^{\prime}, \nabla\right)$ is also naturally enriched to a TERP structure denoted by $\operatorname{Gr}_{\mathfrak{a}}\left(H, H_{\mathbb{R}}^{\prime}, \nabla, P, w\right)$.

Another formulation. In [12, a compatibility of real structure and Stokes structure is formulated in a slightly different way. Let us check that it is equivalent to the above. For simplicity, we consider the case in which $X$ is a point.

Let $H$ be a vector bundle on $\mathbb{C}_{\lambda}$ with a meromorphic flat connection $\nabla: H \rightarrow$ $H \otimes \Omega_{\mathbb{C}_{\lambda}}^{1}(* 0)$ such that $H$ requires no ramification with the good set of irregular values $\operatorname{Irr}(\nabla) \subset \lambda^{-1} \mathbb{C}$. Take $\theta_{0} \in \mathbb{R}$ such that $\operatorname{Re}(\mathfrak{a}-\mathfrak{b})\left(r e^{\sqrt{-1} \theta_{0}}\right) \neq 0$ for any distinct $\mathfrak{a}, \mathfrak{b} \in \operatorname{Irr}(\nabla)$. Take a sufficiently small $\epsilon>0$, and consider the sector

$$
\mathcal{S}:=\left\{r e^{\sqrt{-1} \theta} \mid \theta_{0}-\epsilon \leq \theta \leq \theta_{0}+\pi+\epsilon\right\} .
$$

Let $\overline{\mathcal{S}}$ denote the closure of $\mathcal{S}$ in the real blow up $\widetilde{\mathbb{C}}_{\lambda}(0) \rightarrow \mathbb{C}_{\lambda}$ along 0 . Let $\mathcal{Z}:=\overline{\mathcal{S}} \cap \pi^{-1}(0)$. As a version of the Hukuhara-Turrittin theorem, it is well known that we have a unique flat decomposition

$$
(H, \nabla)_{\mid \overline{\mathcal{S}}}=\bigoplus_{\mathfrak{a} \in \operatorname{Irr}(\nabla)}\left(H_{\mathfrak{a}, \mathcal{S}}, \nabla_{\mathfrak{a}, \mathcal{S}}\right)
$$

such that the restriction of 8.1 to $\widehat{\mathcal{Z}}$ is the same as the pull back of the irregular decomposition of $H_{\mid \widehat{0}}$.

Assume that the flat bundle $(H, \nabla)_{\mid \mathbb{C}_{\lambda}^{*}}$ is equipped with a real structure, i.e., a $\mathbb{C}$-anti-linear flat involution $\kappa: H \rightarrow H$. In other words, $(H, \nabla, \kappa)$ is a TER structure. In Section 8 of 12 , the real structure and the Stokes structure are defined to be compatible if $\kappa\left(H_{\mathfrak{a}, \mathcal{S}}\right)=H_{\mathfrak{a}, \mathcal{S}}$ for any $\mathfrak{a} \in \operatorname{Irr}(\nabla)$ and any $\mathcal{S}$ as above.

If a small sector $S$ is contained in $\mathcal{S}$, the restriction of 8.1 to $S$ gives a splitting of $\mathcal{F}^{S}$. Hence, if $H_{\mathfrak{a}, \mathcal{S}}$ are preserved by $\kappa$ for any a, the filtration $\mathcal{F}^{S}$ is also preserved by $\kappa$. Let $S_{1}$ and $S_{2}$ be small sectors containing the rays $\left\{r e^{\sqrt{-1} \theta_{0}}\right.$ | $r>0\}$ and $\left\{-r e^{\sqrt{-1} \theta_{0}} \mid r>0\right\}$, respectively. Then $\mathfrak{a} \leq_{S_{1}} \mathfrak{b}$ if and only if $\mathfrak{a} \geq_{S_{2}} \mathfrak{b}$. By the parallel transport on $\mathcal{S}$, the flat bundle $H_{\mid \mathcal{S}}$ is trivialized, and we can observe that $H_{\mathfrak{a}, \mathcal{S}}=\mathcal{F}_{\mathfrak{a}}^{S_{1}} \cap \mathcal{F}_{\mathfrak{a}}^{S_{2}}$. Hence, if $\mathcal{F}_{\mathfrak{a}}^{S_{i}}(i=1,2)$ are preserved by $\kappa, H_{\mathfrak{a}, \mathcal{S}}$ is also preserved by $\kappa$. The equivalence of the two notions of compatibility follows from these considerations.

8.1.2. Two Stokes filtrations of integrable twistor structures. Let $\left(V, \widetilde{\mathbb{D}}^{\triangle}\right)$ be a variation of integrable twistor structure over $\mathbb{P}^{1} \times X$. It is obtained as the gluing of a $T E$-structure $\left(V_{0}, \widetilde{\mathbb{D}}_{0}^{f}\right)$ on $\mathcal{X}:=\mathbb{C}_{\lambda} \times X$ and a $\widetilde{T} E$-structure $\left(V_{\infty}, \widetilde{\mathbb{D}}_{\infty}^{\dagger}\right)$ on $\mathcal{X}^{\dagger}:=\mathbb{C}_{\mu} \times X^{\dagger}$. We set $\mathcal{X}^{0}:=\{0\} \times X \subset \mathcal{X}$ and $\mathcal{X}^{\dagger 0}:=\{0\} \times X^{\dagger} \subset \mathbb{C}_{\mu} \times X^{\dagger}$. 
Definition 8.1. We say that $\left(V, \widetilde{\mathbb{D}}^{\Delta}\right)$ is unramifiedly pseudo-good if both $\left(V_{0}, \widetilde{\mathbb{D}}_{0}^{f}\right)$ and $\left(V_{\infty}, \widetilde{\mathbb{D}}_{\infty}^{\dagger f}\right)$ are unramifiedly pseudo-good. In that case, let $\operatorname{Irr}\left(\widetilde{\mathbb{D}}_{0}^{f}\right)$ and $\operatorname{Irr}\left(\widetilde{\mathbb{D}}_{\infty}^{\dagger f}\right)$ denote the sets of irregular values of $\widetilde{\mathbb{D}}_{0}^{f}$ and $\widetilde{\mathbb{D}}_{\infty}^{\dagger f}$, respectively.

If $X$ is a point, we also say that $\left(V, \widetilde{\mathbb{D}}^{\Delta}\right)$ requires no ramification.

Definition 8.2. Assume $\left(V, \widetilde{\mathbb{D}}^{\triangle}\right)$ is unramifiedly pseudo-good.

- We say that the sets of irregular values of $\left(V, \widetilde{\mathbb{D}}^{\triangle}\right)$ are compatible if $\operatorname{Irr}\left(\widetilde{\mathbb{D}}_{0}^{f}\right)$ and $\operatorname{Irr}\left(\widetilde{\mathbb{D}}_{\infty}^{f}\right)$ bijectively correspond by $\mathfrak{a} \leftrightarrow \overline{\gamma^{*} \mathfrak{a}}$.

- We say that $\left(V, \widetilde{\mathbb{D}}^{\triangle}\right)$ has compatible Stokes structures if the following holds:

- The sets of irregular values of $\left(V, \widetilde{\mathbb{D}}^{\triangle}\right)$ are compatible.

- For a small sector $S$ of $\mathcal{X}-\mathcal{X}^{0}$, we have the Stokes filtration $\mathcal{F}^{S}$ of $\left(V_{0}, \widetilde{\mathbb{D}}_{0}^{f}\right)$. We also have the Stokes filtration $\mathcal{F}^{\gamma(S)}$ of $\left(V_{\infty}, \widetilde{\mathbb{D}}_{\infty}^{f}\right)$, where we regard $\gamma(S)$ as a small sector of $\mathcal{X}^{\dagger}-\mathcal{X}^{\dagger 0}$. Then $\mathcal{F}^{S}$ and $\mathcal{F}^{\gamma(S)}$ are the same under the parallel transport along any rays connecting $S$ and $\gamma(S)$.

Remark 28. In the above definition, a ray means a line

$$
\left\{\left(t e^{\sqrt{-1} \varphi}, P\right) \mid 0<t<\infty\right\}
$$

in $\mathbb{C}_{\lambda}^{*} \times\{P\} \subset \mathbb{C}_{\lambda}^{*} \times X$. We say that it connects $S$ and $\gamma(S)$ if (i) $\left(t e^{\sqrt{-1} \varphi}, P\right)$ is contained in $S$ for any sufficiently small $t$, (ii) $\left(t e^{\sqrt{-1} \varphi}, P\right)$ is contained in $\gamma(S)$ for any sufficiently large $t$.

Lemma 8.1. If $\left(V, \widetilde{\mathbb{D}}^{\Delta}\right)$ is equipped with either a real structure $\kappa$ or a perfect pairing $\mathcal{S}$ of weight $w$, then the irregular values of $\widetilde{\mathbb{D}}_{0}^{f}$ and $\widetilde{\mathbb{D}}_{\infty}^{f}$ are compatible.

Proof. We have $\operatorname{Irr}\left(\gamma^{*} \widetilde{\mathbb{D}}_{\infty}^{f}\right)=\left\{\overline{\gamma^{*} \mathfrak{a}} \mid \mathfrak{a} \in \operatorname{Irr}\left(\widetilde{\mathbb{D}}_{\infty}^{f}\right)\right\}$. If $\left(V, \widetilde{\mathbb{D}}^{\Delta}\right)$ is equipped with a real structure, $\gamma^{*}\left(V_{\infty}, \widetilde{\mathbb{D}}_{\infty}^{f}\right) \simeq\left(V_{0}, \widetilde{\mathbb{D}}_{0}^{f}\right)$. Hence, the irregular values of $\widetilde{\mathbb{D}}_{0}^{f}$ and $\widetilde{\mathbb{D}}_{\infty}^{f}$ are compatible.

We have $\operatorname{Irr}\left(\sigma^{*} \widetilde{\mathbb{D}}_{\infty}^{f}\right)=\left\{\overline{\sigma^{*} \mathfrak{a}} \mid \mathfrak{a} \in \operatorname{Irr}\left(\widetilde{\mathbb{D}}_{\infty}^{f}\right)\right\}$. Note that every $\mathfrak{a} \in \operatorname{Irr}\left(\widetilde{\mathbb{D}}_{\infty}^{f}\right)$ is of the form $\mu^{-1} \mathfrak{a}^{\prime}$, where $\mathfrak{a}^{\prime}$ is a holomorphic function on $X^{\dagger}$. Hence, $\overline{\sigma^{*} \mathfrak{a}}=-\overline{\gamma^{*} \mathfrak{a}}$. If $\left(V, \widetilde{\mathbb{D}}_{f}^{\Delta}\right)$ is equipped with a perfect pairing, $\left(V_{0}, \widetilde{\mathbb{D}}_{0}^{f}\right)$ is isomorphic to the dual of $\sigma^{*}\left(V_{\infty}, \widetilde{\mathbb{D}}_{\infty}^{f}\right)$. Therefore, the irregular values of $\widetilde{\mathbb{D}}_{0}^{f}$ and $\widetilde{\mathbb{D}}_{\infty}^{f}$ are compatible.

If $\left(V, \widetilde{\mathbb{D}}^{\Delta}\right)$ is unramifiedly pseudo-good, by taking $\mathrm{Gr}$ with respect to the Stokes filtrations, we obtain a $T E$-structure $\operatorname{Gr}_{\mathfrak{a}}\left(V_{0}, \widetilde{\mathbb{D}}_{0}^{f}\right)$ on $\mathcal{X}$ for $\mathfrak{a} \in \operatorname{Irr}\left(\widetilde{\mathbb{D}}_{0}^{f}\right)$, and a $\widetilde{T} E$-structure $\operatorname{Gr}_{\mathfrak{b}}\left(V_{\infty}, \widetilde{\mathbb{D}}_{\infty}^{f}\right)$ on $\mathcal{X}^{\dagger}$ for $\mathfrak{b} \in \operatorname{Irr}\left(\widetilde{\mathbb{D}}_{\infty}^{f}\right)$. If $\left(V, \widetilde{\mathbb{D}}^{\Delta}\right)$ has compatible Stokes structures, we have the naturally induced isomorphism

$$
\operatorname{Gr}_{\mathfrak{a}}\left(V_{0}, \widetilde{\mathbb{D}}_{0}^{f}\right)_{\mid \mathcal{X}-\mathcal{X}^{0}} \simeq \operatorname{Gr}_{\bar{\gamma}^{*} \mathfrak{a}}\left(V_{\infty}, \widetilde{\mathbb{D}}_{\infty}^{f}\right)_{\mid \mathcal{X}^{\dagger}-\mathcal{X}^{\dagger 0}} .
$$


Hence, we obtain a variation of integrable twistor structure $\operatorname{Gr}_{\mathfrak{a}}\left(V, \widetilde{\mathbb{D}}^{\triangle}\right)$ for each $\mathfrak{a} \in \operatorname{Irr}\left(\widetilde{\mathbb{D}}_{0}^{f}\right)$ as their gluing. We have the following functoriality (Lemma 5.7).

Lemma 8.2. Let $\left(V^{(a)}, \widetilde{\mathbb{D}}^{(a) \triangle}\right)$ be unramifiedly pseudo-good. Assume that (i) $\left(V^{(a)}, \widetilde{\mathbb{D}}^{(a)} \triangle\right)(a=1,2)$ have compatible Stokes filtrations, (ii) the union $\mathcal{I}:=$ $\operatorname{Irr}\left(\widetilde{\mathbb{D}}_{0}^{(1) f}\right) \cup \operatorname{Irr}\left(\widetilde{\mathbb{D}}_{0}^{(2) f}\right)$ is good. Then a morphism $\left(V^{(1)}, \widetilde{\mathbb{D}}^{(1)} \triangle\right) \rightarrow\left(V^{(2)}, \widetilde{\mathbb{D}}^{(2)} \triangle\right)$ induces $\operatorname{Gr}_{\mathfrak{a}}\left(V^{(1)}, \widetilde{\mathbb{D}}^{(1)} \triangle\right) \rightarrow \operatorname{Gr}_{\mathfrak{a}}\left(V^{(2)}, \widetilde{\mathbb{D}}^{(2) \triangle}\right)$ for each $\mathfrak{a} \in \mathcal{I}$.

We have the natural isomorphisms

$$
\gamma^{*} \operatorname{Gr}_{\mathfrak{a}}\left(V, \widetilde{\mathbb{D}}^{\triangle}\right) \simeq \operatorname{Gr}_{\mathfrak{a}}\left(\gamma^{*}\left(V, \widetilde{\mathbb{D}}^{\triangle}\right)\right), \quad \sigma^{*} \operatorname{Gr}_{\mathfrak{a}}\left(V, \widetilde{\mathbb{D}}^{\triangle}\right) \simeq \operatorname{Gr}_{-\mathfrak{a}}\left(\sigma^{*}\left(V, \widetilde{\mathbb{D}}^{\triangle}\right)\right) .
$$

The following lemma follows from functoriality.

Lemma 8.3. Assume that $\left(V, \widetilde{\mathbb{D}}^{\triangle}\right)$ has compatible Stokes structures. If $\left(V, \widetilde{\mathbb{D}}^{\triangle}\right)$ is equipped with a real structure (resp. a perfect pairing of weight w), each $\operatorname{Gr}_{\mathfrak{a}}\left(V, \widetilde{\mathbb{D}}^{\triangle}\right.$ ) is also equipped with an induced real structure (resp. an induced perfect pairing of weight $w)$.

Lemma 8.4. Let $\left(H, H_{\mathbb{R}}^{\prime}, \nabla, P^{\prime}, w\right)$ be a variation of TERP structure, and let $\left(V, \widetilde{\mathbb{D}}^{\triangle}, \mathcal{S}, \kappa, w\right)$ be the corresponding variation of twistor-TERP structure. (See Subsection 2.1.8.) Assume that $\left(H, H_{\mathbb{R}}^{\prime}, \nabla, P^{\prime}, w\right)$ is unramifiedly pseudo-good, or equivalently, $\left(V, \widetilde{\mathbb{D}}^{\triangle}, \mathcal{S}, \kappa, w\right)$ is unramifiedly pseudo-good.

- The real structure and the Stokes structure of $(H, \nabla)$ are compatible if and only if $\left(V, \widetilde{\mathbb{D}}^{\triangle}\right)$ has compatible Stokes structures.

- Assume that the real structures and the Stokes structures are compatible. Then $\operatorname{Gr}_{\mathfrak{a}}\left(V, \widetilde{\mathbb{D}}{ }^{\triangle}, \mathcal{S}, \kappa, w\right)$ is the variation of twistor-TERP structure corresponding to $\operatorname{Gr}_{\mathfrak{a}}\left(H, H_{\mathbb{R}}^{\prime}, \nabla, P^{\prime}, w\right)$.

Proof. Note that the Stokes filtration of $\gamma^{*}(H, \nabla)$ on $\gamma^{*}(S)$ is given by the composite of the conjugate with respect to $H_{\mathbb{R}}^{\prime}$ and the parallel transport along the rays connecting $S$ and $\gamma(S)$, with the change of the index sets from $\operatorname{Irr}(\nabla)$ to $\left\{\overline{\gamma^{*} \mathfrak{a}} \mid \mathfrak{a} \in \operatorname{Irr}(\nabla)\right\}$. Hence, the first claim follows.

Let us consider the second claim. We may assume that $\left(H, H_{\mathbb{R}}^{\prime}, \nabla, P^{\prime}, w\right)$ is obtained from $(V, \nabla, \mathcal{S}, \kappa, w)$ by the procedure explained in Subsection 2.1.8. By construction, we have $\operatorname{Gr}_{\mathfrak{a}}(H, \nabla)=\operatorname{Gr}_{\mathfrak{a}}\left(V_{0}, \nabla_{0}\right)$. For comparison of induced real structures and pairings, we have only to consider the case in which $X$ is a point.

Let us compare the induced real structures. The flat real structure of $H^{\prime}$ is obtained as the composite

$$
\bar{H}_{\mid \lambda} \stackrel{\text { parallel transport }}{\longrightarrow} \bar{H}_{\mid \bar{\lambda}^{-1}} \stackrel{\kappa_{\mid \lambda}}{\longrightarrow} H_{\mid \lambda} .
$$


Hence, we have the following factorization of the real structure on $\operatorname{Gr}_{\mathfrak{a}}(H)_{\mid \lambda}$ obtained as Gr of the Stokes filtration:

$$
{\overline{\mathrm{Gr}_{\mathfrak{a}}(H)}}_{\mid \lambda} \stackrel{\text { parallel transport }}{\longrightarrow}{\overline{\mathrm{Gr}_{\mathfrak{a}}(H)}}_{\mid \bar{\lambda}^{-1}} \stackrel{\mathrm{Gr}_{\mathfrak{a}}(\kappa)_{\mid \lambda}}{\longrightarrow} \operatorname{Gr}_{\mathfrak{a}}(H)_{\mid \lambda} .
$$

It is the same as the real structure induced by $\operatorname{Gr}_{\mathfrak{a}}(\kappa)$ on $\operatorname{Gr}_{\mathfrak{a}}(V, \nabla)$.

Let $P: H \otimes j^{*} H \rightarrow \mathcal{O}_{\mathbb{C}_{\lambda}}$ be the pairing induced by $\kappa$ and $\mathcal{S}$ as in (2.5), whose restriction to $H^{\prime}$ is $P^{\prime}$. Let $S$ be a small sector in $\mathbb{C}_{\lambda}^{*}$. We have the following factorization of $(\sqrt{-1})^{-w} P_{\mid S}$ :

$$
\begin{aligned}
\mathcal{F}_{\mathfrak{a}}^{S}(H) \otimes j^{*} \mathcal{F}_{\mathfrak{b}}^{j(S)}(H)=\mathcal{F}_{\mathfrak{a}}^{S}\left(V_{0}\right) \otimes \sigma^{*} \gamma^{*} \mathcal{F}_{\mathfrak{b}}^{j(S)}\left(V_{0}\right) \\
\stackrel{1 \otimes \sigma^{*} \kappa}{\longrightarrow} \mathcal{F}_{\mathfrak{a}}^{S}\left(V_{0}\right) \otimes \sigma^{*} \mathcal{F}_{\frac{\sigma(S)}{\gamma^{*}(\mathfrak{b})}}\left(V_{\infty}\right) \stackrel{\mathcal{S}}{\longrightarrow} \mathcal{O}_{S}
\end{aligned}
$$

The restriction to $\mathcal{F}_{\mathfrak{a}}^{S}(H) \otimes j^{*} \mathcal{F}_{\mathfrak{b}}^{j(S)}(H)$ is 0 unless $\mathfrak{a}-\mathfrak{b} \geq_{S} 0$. The induced pairing $(\sqrt{-1})^{-w} P_{\mathfrak{a}}$ for $\operatorname{Gr}_{\mathfrak{a}}\left(V_{0}\right)$ is factorized as follows:

$\mathrm{Gr}_{\mathfrak{a}}\left(V_{0}\right)_{\mid S} \otimes j^{*} \mathrm{Gr}_{\mathfrak{a}}\left(V_{0}\right)_{\mid j(S)} \stackrel{1 \otimes \sigma^{*} \mathrm{Gr}_{\mathfrak{a}} \kappa}{\longrightarrow} \operatorname{Gr}_{\mathfrak{a}}\left(V_{0}\right)_{\mid S} \otimes \sigma^{*} \operatorname{Gr}_{\gamma^{*}(\mathfrak{a})}\left(V_{\infty}\right)_{\mid \sigma(S)} \stackrel{\mathrm{Gr}_{\mathfrak{a}} \mathcal{S}}{\longrightarrow} \mathcal{O}_{S}$.

Hence, it is the same as the pairing induced by $\operatorname{Gr}_{\mathfrak{a}}(V, \nabla, \mathcal{S}, \kappa)$. Thus, the proof of Lemma 8.4 is finished.

8.1.3. Preliminaries on pull back. We set $X:=\mathbb{C}_{z}, D=\{0\}, \mathcal{X}:=\mathbb{C}_{\lambda} \times X$, $\mathcal{D}:=\mathbb{C}_{\lambda} \times D$ and $W:=\mathcal{D} \cup(\{0\} \times X)$. Let $\pi: \widetilde{\mathcal{X}}(W) \rightarrow \mathcal{X}$ be a real blow up of $\mathcal{X}$ along $W$. Let $\pi_{1}: \widetilde{\mathbb{C}}_{\lambda}(0) \rightarrow \mathbb{C}_{\lambda}$ be the real blow up of $\mathbb{C}_{\lambda}$ along $\{0\}$. Let $\phi_{0}: \mathcal{X} \rightarrow \mathbb{C}_{\lambda}$ be given by $\phi_{0}(\lambda, z)=\lambda z$. It induces a map $\widetilde{\phi}_{0}: \widetilde{\mathcal{X}}(W) \rightarrow \widetilde{\mathbb{C}}_{\lambda}(0)$.

Let $H$ be a vector bundle on $\mathbb{C}_{\lambda}$ with a meromorphic flat connection $\nabla$ : $H \rightarrow H \otimes \Omega_{\mathbb{C}_{\lambda}}^{1}(* 0)$ such that $(H, \nabla)$ requires no ramification with the good set of irregular values $\mathcal{I} \subset \lambda^{-1} \mathbb{C}$. Let $\mathfrak{V}$ denote the flat bundle on $\widetilde{\mathbb{C}}_{\lambda}(0)$ associated to $H_{\mid \mathbb{C}_{\lambda}^{*}}$. For each $Q \in \pi_{1}^{-1}(0)$, we have the Stokes filtration $\mathcal{F}^{Q}$ of $\mathfrak{V}_{\mid Q}$ for the meromorphic prolongment $H$. (See Subsection 5.1.5.) We can naturally regard $\widetilde{\phi}_{0}^{*} \mathfrak{V}$ as the flat bundle on $\widetilde{\mathcal{X}}(W)$ associated to $\left(\phi_{0}^{*} H\right)_{\mid \mathcal{X}}-W$.

\section{Lemma 8.5.}

- $\phi_{0}^{*}(H, \nabla)$ is unramifiedly pseudo-good on the level $\boldsymbol{m}=(-1,-1)$. (See Subsection 5.1.3.) The set of irregular values is $\phi_{0}^{*} \mathcal{I}:=\left\{\phi_{0}^{*} \mathfrak{a} \mid \mathfrak{a} \in \mathcal{I}\right\}$.

- For each $P \in \pi^{-1}(W)$, the Stokes filtration $\mathcal{F}^{P}$ of $\widetilde{\phi}_{0}^{*}(\mathfrak{V})_{\mid P}$ for $\phi_{0}^{*} H$ is the pull back of the Stokes filtration of $\mathfrak{V}_{\mid \widetilde{\phi}_{0}(P)}$.

- We have the natural isomorphism $\phi_{0}^{*} \operatorname{Gr}_{\mathfrak{a}}(H) \simeq \operatorname{Gr}_{\phi_{0}^{*} \mathfrak{a}}\left(\phi_{0}^{*} H\right)$.

Proof. We have the decomposition $(H, \nabla)_{\mid \widehat{0}}=\bigoplus_{\mathfrak{a} \in \mathcal{I}}\left(H_{\mathfrak{a}}, \widehat{\nabla}_{\mathfrak{a}}\right)$, where $\widehat{\nabla}_{\mathfrak{a}}-d \mathfrak{a}$ are regular. It induces the decomposition of $\phi_{0}^{*}(H, \nabla)_{\mid} \widehat{W}$. Hence, the first claim is clear. 
We set $Q:=\widetilde{\phi}_{0}(P)$. Note that the orders $\leq_{Q}$ and $\leq_{P}$ are the same under the identification $\mathcal{I} \simeq \phi_{0}^{*} \mathcal{I}$. Let $H_{1} \supset H$ be an unramifiedly good lattice. Thus, $\phi_{0}^{*} H_{1}$ is an unramifiedly good lattice. We take a small sector $S_{Q} \in \mathcal{M S}\left(Q, \mathbb{C}_{\lambda}^{*}, \mathcal{I}\right)$ such that there exists the Stokes filtration $\mathcal{F}^{S_{Q}}$ of $H_{1 \mid \bar{S}_{Q}}$. We take a small multisector $S_{P} \in \mathcal{M S}\left(P, \mathcal{X}-W, \phi_{0}^{*} \mathcal{I}\right)$ such that $\phi_{0}\left(S_{P}\right) \subset S_{Q}$. Thus, we obtain the filtration $\widetilde{\phi}_{0}^{*} \mathcal{F}^{S_{Q}}$ of $\phi_{0}^{*}\left(H_{1}\right)_{\bar{S}_{P}}$ indexed by $\left(\phi_{0}^{*} \mathcal{I}, \leq_{P}\right)$. It gives the Stokes filtration of $\phi_{0}^{*}\left(H_{1}\right)_{\mid \bar{S}_{P}}$, which follows from the characterization in Proposition 5.1. Since the filtration of $\widetilde{\phi}_{0}^{*}(\mathfrak{V})_{\mid P}$ induced by $\widetilde{\phi}_{0}^{*} \mathcal{F}^{S_{Q}}$ is the same as the pull back of $\mathcal{F}^{Q}$ on $\mathfrak{V}_{\mid Q}$, we obtain the second claim. Note also that the Stokes filtration of $\phi_{0}^{*}(H)_{\mid \bar{S}_{P}}$ is given by the pull back of the Stokes filtration of $H_{\mid \bar{S}_{Q}}$.

Let $S_{P}$ be a small multi-sector as above. By the above compatibility of the Stokes filtrations and Lemma 5.9 , we obtain the natural isomorphisms

$$
\phi_{0}^{*}\left(\operatorname{Gr}_{\mathfrak{a}}(H)\right)_{\mid \bar{S}_{P}} \simeq \operatorname{Gr}_{\phi_{0}^{*} \mathfrak{a}}\left(\phi_{0}^{*} H\right)_{\mid \bar{S}_{P}} .
$$

By varying $S_{P}$ and gluing them, we obtain

$$
\phi_{0}^{*}\left(\operatorname{Gr}_{\mathfrak{a}}(H)\right)_{\mid \widetilde{\mathcal{U}}(W)} \simeq \operatorname{Gr}_{\phi_{0}^{*} \mathfrak{a}}\left(\phi_{0}^{*} H\right)_{\mid \widetilde{\mathcal{U}}(W)},
$$

where $\mathcal{U}$ is a neighbourhood of $W$, and $\widetilde{\mathcal{U}}(W)$ denotes the real blow up of $\mathcal{U}$ along $W$. By using flatness, this isomorphism extends to $\phi_{0}^{*}\left(\operatorname{Gr}_{\mathfrak{a}}(H)\right)_{\mid \widetilde{\mathcal{X}}(W)} \simeq$ $\operatorname{Gr}_{\phi_{0}^{*} \mathfrak{a}}\left(\phi_{0}^{*} H\right)_{\mid \widetilde{\mathcal{X}}(W)}$. Hence, we obtain an isomorphism on $\mathcal{X}$.

8.1.4. Rescaling and HS-orbits. We recall a rescaling construction from 10 and [12. See also [26]. We set $X:=\mathbb{C}_{z}, D=\{0\}$ and $X^{*}:=X-D$. For $R>0$, we set $X(R):=\{z \in X|| z \mid<R\}$ and $X^{*}(R):=X(R) \cap X^{*}$. We set $\mathcal{X}:=\mathbb{C}_{\lambda} \times X$. We use the symbols $\mathcal{X}^{*}, \mathcal{D}, \mathcal{X}(R)$ and $\mathcal{X}^{*}(R)$ in similar meanings. Let $\phi_{0}: \mathcal{X} \rightarrow \mathbb{C}_{\lambda}$ be given by $\phi_{0}(\lambda, z)=\lambda z$. The restriction to $\mathcal{X}^{*}$ is denoted by $\psi_{0}$.

TERP structure. We consider only TERP structures of weight 0 . Hence, we do not specify weights. Let $\left(H, H_{\mathbb{R}}^{\prime}, \nabla, P\right)$ be a TERP structure. Hertling and Sevenheck studied the variation of TERP structure $\psi_{0}^{*}\left(H, H_{\mathbb{R}}^{\prime}, \nabla, P\right)$ on $X^{*}$. If there exists an $R>0$ such that $\psi_{0}^{*}\left(H, H_{\mathbb{R}}^{\prime}, \nabla, P\right)_{\mid X^{*}(R)}$ is pure and polarized, the variation is called an $H S$-orbit (Hertling-Sevenheck orbit), and we say in this paper that $\left(H, H_{\mathbb{R}}^{\prime}, \nabla, P\right)$ induces an $H S$-orbit.

Remark 29. An HS-orbit is called a "nilpotent orbit" in 12. We use "HS-orbit" to distinguish it from twistor nilpotent orbit. It corresponds to their term "Sabbahorbit".

Lemma 8.6. Assume (i) $(H, \nabla)$ requires no ramification, (ii) the Stokes structure and the real structure of $\left(H, H_{\mathbb{R}}^{\prime}, \nabla\right)$ are compatible. Then: 
- $\psi_{0}^{*}(H, \nabla)$ is unramifiedly pseudo-good. The set of irregular values is given by $\left\{\psi_{0}^{*} \mathfrak{a} \mid \mathfrak{a} \in \operatorname{Irr}(\nabla)\right\}$.

- The real structure and the Stokes structure of $\psi_{0}^{*}(H, \nabla)$ are compatible.

- We have a natural isomorphism

$$
\psi_{0}^{*} \operatorname{Gr}_{\mathfrak{a}}\left(H, H_{\mathbb{R}}^{\prime}, \nabla, P\right) \simeq \operatorname{Gr}_{\psi_{0}^{*} \mathfrak{a}} \psi_{0}^{*}\left(H, H_{\mathbb{R}}^{\prime}, \nabla, P\right) .
$$

Proof. The first two claims follow from Lemma 8.5. To show the third claim, we have only to compare the induced flat pairings. This can be done directly, or by considering the restriction to $\mathbb{C}_{\lambda} \times\{1\}$.

The lemma means in particular that an HS-orbit is wild. Note that there is a non-wild variation of pure polarized TERP structure on $X^{*}(R)$. For example, consider the example in Subsection 2.2.1 with a transcendental holomorphic function $\mathfrak{a}$ on $X^{*}(R)$.

Integrable twistor structure. We set $\mathcal{X}^{\dagger}:=\mathbb{C}_{\mu} \times X^{\dagger}, \mathcal{D}^{\dagger}:=\mathbb{C}_{\mu} \times D^{\dagger}, \mathcal{X}^{* \dagger}:=$ $\mathcal{X}^{\dagger}-\mathcal{D}^{\dagger}$ and $W^{\dagger}:=\mathcal{D}^{\dagger} \cup\left(\{0\} \times X^{\dagger}\right)$. Let $\phi_{\infty}: \mathcal{X}^{\dagger} \rightarrow \mathbb{C}_{\mu}$ be given by $\phi_{\infty}(\mu, z)=\mu \bar{z}$. The restriction to $\mathcal{X}^{* \dagger}$ is denoted by $\psi_{\infty}$.

Let $(V, \nabla)$ be an integrable twistor structure on $\mathbb{P}^{1}$ which requires no ramification. It is obtained as the gluing of $\left(V_{0}, \nabla_{0}\right)$ and $\left(V_{\infty}, \nabla_{\infty}\right)$. The gluing is denoted by $g: V_{0 \mid \mathbb{C}_{\lambda}^{*}} \simeq V_{\infty \mid \mathbb{C}_{\mu}^{*}}$, which is flat with respect to $\nabla$.

We set $\operatorname{HS}(V)_{0}:=\psi_{0}^{*}\left(V_{0}\right)$ and $\operatorname{HS}(V)_{\infty}:=\psi_{\infty}^{*}\left(V_{\infty}\right)$. They are naturally equipped with a $T E$-structure $\operatorname{HS}(\nabla)_{0}$ and a $\widetilde{T} E$-structure $\operatorname{HS}(\nabla)_{\infty}$. Note that $\mathrm{HS}(V, \nabla)_{0}$ and $\mathrm{HS}(V, \nabla)_{\infty}$ are unramifiedly pseudo-good. Let us construct a

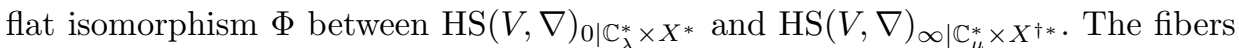
$\operatorname{HS}(V)_{0 \mid(\lambda, z)}$ and $\operatorname{HS}(V)_{\infty \mid(\mu, z)}$ are naturally identified with $V_{0 \mid \lambda z}$ and $V_{\infty \mid \mu \bar{z}}$, respectively. If $\lambda=\mu^{-1}$, we have $(\lambda z)^{-1}=\mu \bar{z}|z|^{-2}$. Hence, we have an isomorphism $\Phi_{(\lambda, z)}: \operatorname{HS}(V)_{0 \mid(\lambda, z)} \simeq \operatorname{HS}(V)_{\infty \mid\left(\lambda^{-1}, z\right)}$ induced by the gluing $g$ with the parallel transport along the segments connecting $\lambda^{-1} \bar{z}$ and $\lambda^{-1} \bar{z}|z|^{-2}$. Thus, we obtain the isomorphism $\Phi$ as desired.

Let $\operatorname{HS}(V, \nabla)$ denote the variation of integrable twistor structure obtained as the gluing of $\operatorname{HS}(V, \nabla)_{0}$ and $\operatorname{HS}(V, \nabla)_{\infty}$. The following lemma is clear from the construction and the functoriality (Lemma 5.7).

\section{Lemma 8.7.}

- Let $F:\left(V^{(1)}, \nabla^{(1)}\right) \rightarrow\left(V^{(2)}, \nabla^{(2)}\right)$ be a morphism of integrable pure twistor structures. Then we have the induced morphism

$$
\operatorname{HS}(F): \operatorname{HS}\left(V^{(1)}, \nabla^{(1)}\right) \rightarrow \operatorname{HS}\left(V^{(2)}, \nabla^{(2)}\right)
$$


- Let $f$ be $\gamma$ or $\sigma$. Then $\mathrm{HS} \circ f^{*}(V, \nabla)$ is naturally isomorphic to the pull back $f^{*} \operatorname{HS}(V, \nabla)$.

By the above lemma, a real structure $\kappa$ of $(V, \nabla)$ induces a real structure $\operatorname{HS}(\kappa)$ of $\operatorname{HS}(V, \nabla)$. Since we have the natural isomorphism $\operatorname{HS}(\mathbb{T}(0)) \simeq \mathbb{T}(0)_{X^{*}}$, a paring $\mathcal{S}$ of $(V, \nabla)$ with weight 0 induces a pairing $\operatorname{HS}(\mathcal{S})$ of $\operatorname{HS}(V, \nabla)$ with weight 0 . Hence, an integrable twistor structure with a pairing $(V, \nabla, \mathcal{S})$ induces $\operatorname{HS}(V, \nabla, \mathcal{S})$ on $\mathbb{P}^{1} \times X^{*}$, and if $(V, \nabla, \mathcal{S})$ is equipped with a real structure, $\operatorname{HS}(V, \nabla, \mathcal{S})$ is also equipped with a naturally induced real structure.

Lemma 8.8. If $(V, \nabla)$ has compatible Stokes structures, $\operatorname{HS}(V, \nabla)$ also has compatible Stokes structures, and we have the natural isomorphism

$$
\operatorname{HSGr}_{\mathfrak{a}}(V, \nabla) \simeq \operatorname{Gr}_{\psi_{0}^{*} \mathfrak{a}} \operatorname{HS}(V, \nabla) .
$$

If $(V, \nabla)$ is equipped with a pairing of weight 0 (resp. a real structure), (8.3) preserves the induced pairings (resp. real structures).

Proof. This follows from Lemma 8.5 .

Lemma 8.9. Let $\left(H, H_{\mathbb{R}}^{\prime}, \nabla, P^{\prime}\right)$ be a TERP structure, and $(V, \nabla, \mathcal{S}, \kappa)$ be the corresponding twistor-TERP structure. Then $\operatorname{HS}(V, \nabla, \mathcal{S}, \kappa)$ is the variation of twistor-TERP structure corresponding to $\psi_{0}^{*}\left(H, H_{\mathbb{R}}^{\prime}, \nabla, P^{\prime}\right)$.

Proof. By construction, we have the natural isomorphism $\operatorname{HS}(V, \nabla)_{0} \simeq(H, \nabla)$. We have only to compare the induced real structures and pairings on them. Since they are flat, we have only to compare them on the fiber over $z=1$. Then the claim is clear.

If there exists an $R>0$ such that $\operatorname{HS}(V, \nabla, \mathcal{S})_{\mid \mathbb{P}^{1} \times X^{*}(R)}$ is pure and polarized, it is called a twistor $H S$-orbit, and we say that $(V, \nabla, \mathcal{S})$ induces a twistor HS-orbit.

\section{$\S 8.2$. Reduction of wild HS-orbits}

8.2.1. Statement. We use the notation in Subsection 8.1.4. Let $(V, \nabla)$ be an integrable twistor structure with a perfect pairing $\mathcal{S}$ of weight 0 , which requires no ramification. Assume that $(V, \nabla, \mathcal{S})$ induces a twistor HS-orbit on $\mathbb{P}^{1} \times X^{*}(R)$ for some $R>0$. We obtain the underlying unramifiedly good wild harmonic bundle $\left(E, \bar{\partial}_{E}, \theta, h\right)$ on $X^{*}(R)$ of $\operatorname{HS}(V, \nabla, \mathcal{S})_{\mid \mathbb{P}^{1} \times X^{*}(R)}$, which is unramifiedly good. Let $\mathcal{I}$ denote the set of irregular values of $(V, \nabla)$ at 0 . It is easy to see that

$$
\operatorname{Irr}(\theta)=\left\{(\lambda \cdot \mathfrak{a}(\lambda z))_{\mid \lambda=0} \mid \mathfrak{a}(\lambda) \in \mathcal{I}\right\}=\{\mathfrak{a}(z) \mid \mathfrak{a}(\lambda) \in \mathcal{I}\} \simeq \mathcal{I}
$$

Note that any $\mathfrak{a} \in \mathcal{I}$ is of the form $\alpha / \lambda$ for some $\alpha \in \mathbb{C}$. We will not distinguish them in the following. 
Let $\left(\mathcal{E}^{\triangle}, \mathbb{D}^{\triangle}, \mathcal{S}_{E}\right)$ denote the variation of polarized pure twistor structure associated to $\left(E, \bar{\partial}_{E}, \theta, h\right)$. It is enriched to an integrable one $\left(\mathcal{E}^{\triangle}, \widetilde{\mathbb{D}}^{\triangle}, \mathcal{S}_{E}\right)$. Although it is naturally isomorphic to $\operatorname{HS}(V, \nabla, \mathcal{S})$, it is non-trivial that the natural meromorphic extensions $\mathcal{Q E}_{0}$ and $\phi_{0}^{*}\left(V_{0}\right) \otimes \mathcal{O}_{\mathcal{X}}(* \mathcal{D})$ are isomorphic. Hence, we use the symbol $\left(\mathcal{E}^{\triangle}, \widetilde{\mathbb{D}}^{\triangle}, \mathcal{S}_{E}\right)$ for distinction. By applying the construction in Subsection 7.3.1 to $\left(\mathcal{E}^{\triangle}, \widetilde{\mathbb{D}}^{\triangle}, \mathcal{S}_{E}\right)$, we obtain a wild variation of pure polarized integrable twistor structure $\operatorname{Gr}_{\mathfrak{a}}\left(\mathcal{E}^{\triangle}, \widetilde{\mathbb{D}}^{\triangle}, \mathcal{S}_{E}\right)$ for each $\mathfrak{a} \in \mathcal{I}$. We will prove the following theorem in Subsection 8.2 .2 .

\section{Theorem 8.1.}

- $(V, \nabla)$ has compatible Stokes structures.

- $\operatorname{HS~}_{\mathrm{Gr}_{\mathfrak{a}}}(V, \nabla, \mathcal{S})$ is naturally isomorphic to $\mathrm{Gr}_{\mathfrak{a}}\left(\mathcal{E}^{\triangle}, \widetilde{\mathbb{D}}^{\triangle}, \mathcal{S}_{E}\right)$ for each $\mathfrak{a} \in \mathcal{I}$. In particular, $\operatorname{Gr}_{\mathfrak{a}}(V, \nabla, \mathcal{S})$ induces a twistor $H S$-orbit.

Before going into the proof, we give a consequence.

Corollary 8.1. Let $\left(H, H_{\mathbb{R}}^{\prime}, \nabla, P, 0\right)$ be a TERP structure which requires no ramification. If $\left(H, H_{\mathbb{R}}^{\prime}, \nabla, P, 0\right)$ induces an $H S$-orbit, it is a mixed-TERP structure in the sense of Definition 9.1 of [12].

Proof. Thanks to Theorem 9.3 of $\left[12,\left(H, H_{\mathbb{R}}^{\prime}, \nabla, P, 0\right)\right.$ is a mixed-TERP structure if and only if (i) the real structure and the Stokes structure of $\left(H, H_{\mathbb{R}}^{\prime}, \nabla\right)$ are compatible, (ii) $\operatorname{Gr}_{\mathfrak{a}}\left(H, H_{\mathbb{R}}^{\prime}, \nabla, P, 0\right)$ induces an HS-orbit for each $\mathfrak{a} \in \operatorname{Irr}(\nabla)$. Hence, this corollary follows from Theorem 8.1. Lemma 8.4 and Lemma 8.9 .

The claim of the corollary was established by Hertling and Sevenheck [12] in the case that $(H, \nabla)$ has regular singularity. They also showed the converse of the claim in general.

Remark 30. In their study of the case that $(H, \nabla)$ has regular singularity, Hertling and Sevenheck closely investigated the limiting object. In particular, they showed that the limiting TERP structure is generated by elementary sections, for which the eigenvalues of the new supersymmetric index can be described in terms of the Hodge filtrations of the corresponding mixed Hodge structure.

Even in the irregular case, the limiting object can be obtained from the reduced regular one. Hence, the limit of the eigenvalues of the new supersymmetric index of $\phi_{0}^{*}\left(H, H_{\mathbb{R}}^{\prime}, \nabla, P\right)$ can be described in terms of their mixed Hodge structures.

8.2.2. Proof of Theorem 8.1, We have the natural identifications

$$
\operatorname{HS}(V, \nabla)_{0} \simeq\left(\mathcal{E}, \widetilde{\mathbb{D}}^{f}\right), \quad \operatorname{HS}(V, \nabla)_{\infty} \simeq\left(\mathcal{E}^{\dagger}, \widetilde{\mathbb{D}}^{\dagger} f\right)
$$


We have the locally free $\mathcal{O}_{\mathcal{X}}(* \mathcal{D})$-module

$$
\overline{\mathrm{HS}}(V)_{0}:=\phi_{0}^{*}\left(V_{0}\right) \otimes \mathcal{O}_{\mathcal{X}}(* \mathcal{D}) .
$$

We also have the locally free $\mathcal{O}_{\mathcal{X}^{\dagger}}\left(* \mathcal{D}^{\dagger}\right)$-module

$$
\overline{\operatorname{HS}}(V)_{\infty}:=\phi_{\infty}^{*}\left(V_{\infty}\right) \otimes \mathcal{O}_{\mathcal{X}^{\dagger}}\left(* \mathcal{D}^{\dagger}\right) .
$$

Comparison of $\mathcal{Q E}$ and $\overline{\mathrm{HS}}(V)_{0}$. We would like to show that $\mathcal{Q E}$ and $\overline{\mathrm{HS}}(V)_{0}$ are naturally isomorphic. We set $W:=\mathcal{D} \cup(\{0\} \times X)$. Let $\pi: \widetilde{\mathcal{X}}(W) \rightarrow \mathcal{X}$ be the real blow up of $\mathcal{X}$ along $W$. Let $\mathfrak{V}$ be the flat bundle on $\widetilde{\mathcal{X}}(W)$ associated to $\left(\mathcal{E}, \widetilde{\mathbb{D}}^{f}\right)_{\mid \mathcal{X}-W}$. We set $\phi_{0}^{*} \mathcal{I}:=\left\{\phi_{0}^{*} \mathfrak{a} \mid \mathfrak{a} \in \mathcal{I}\right\}$.

As remarked in Lemma 8.5 $\phi_{0}^{*} H$ is an unramifiedly pseudo-good lattice of $\overline{\mathrm{HS}}(V)_{0} \otimes \mathcal{O}_{\mathcal{X}}(* W)$ on the level $\boldsymbol{m}=(-1,-1)$.

Lemma 8.10. $\mathcal{Q}_{0} \mathcal{E} \subset \mathcal{Q E} \otimes \mathcal{O}_{\mathcal{X}}(* W)$ is an unramifiedly pseudo-good lattice on the level $\boldsymbol{m}=(-1,-1)$ around $(\lambda, z)=(0,0)$.

Proof. We have the meromorphic flat bundle $\operatorname{Gr}_{\mathfrak{a}}(\mathcal{Q E})(* W)$ with the induced connection $\widetilde{\mathbb{D}}_{\mathfrak{a}}^{f}$ on $(\mathcal{X}, W)$ for each $\mathfrak{a} \in \operatorname{Irr}(\theta)$. Put $\nabla_{\mathfrak{a}}^{0}:=\widetilde{\mathbb{D}}_{\mathfrak{a}}^{f}-d(\mathfrak{a}(z) / \lambda)$. The restriction of $\left(\mathrm{Gr}_{\mathfrak{a}}(\mathcal{Q E})(* W), \nabla_{\mathfrak{a}}^{0}\right)$ to $\mathcal{X}-(\{0\} \times X)$ is regular singular by construction. The restriction to $\mathcal{X}-\mathcal{D}$ is also regular singular by Lemma 7.4. Hence, $\left(\operatorname{Gr}_{\mathfrak{a}}(\mathcal{Q E})(* W), \nabla_{\mathfrak{a}}^{0}\right)$ is regular singular.

We have the decomposition

$$
\left(\mathcal{Q}_{0} \mathcal{E}, \mathbb{D}\right)_{\mid \widehat{W}}=\bigoplus_{\mathfrak{a} \in \mathcal{I}}\left(\mathcal{Q}_{0} \widehat{\mathcal{E}}_{\mathfrak{a}}, \widehat{\mathbb{D}}_{\mathfrak{a}}\right)
$$

such that $\widehat{\mathbb{D}}_{\mathfrak{a}}-d_{X} \mathfrak{a}$ are logarithmic. Because we have a natural isomorphism $\mathcal{Q}_{0} \widehat{\mathcal{E}}_{\mathfrak{a}}(* W) \simeq \operatorname{Gr}_{\mathfrak{a}}(\mathcal{Q E})(* W)$, the claim of the lemma follows.

Let $P \in \pi^{-1}(0,0)$. We have the Stokes filtration $\mathcal{F}_{1}^{P}$ of $\mathfrak{V}_{\mid P}$ corresponding to the meromorphic prolongment $\mathcal{Q E} \otimes \mathcal{O}_{\mathcal{X}}(* W)$, and the Stokes filtration $\mathcal{F}_{2}^{P}$ of $\mathfrak{V}_{\mid P}$ corresponding to the meromorphic prolongment $\overline{\mathrm{HS}}(V)_{0} \otimes \mathcal{O}_{\mathcal{X}}(* W)$. (See Subsection 5.1.5 for such filtrations in the pseudo-good case.)

Lemma 8.11. $\mathcal{F}_{1}^{P}=\mathcal{F}_{2}^{P}$.

Proof. Let $S_{P} \in \mathcal{M S}\left(P, \mathcal{X}-W, \phi_{0}^{*} \mathcal{I}\right)$ be a small sector such that the Stokes filtrations $\mathcal{F}_{1}^{S_{P}}$ of $\mathcal{Q E}_{\mid \bar{S}_{P}}$ and $\mathcal{F}_{2}^{S_{P}}$ of $\phi_{0}^{*}(H)_{\mid \bar{S}_{P}}$ both exist. We can take $Q \in$ $\bar{S}_{P} \cap \pi^{-1}(W \backslash \mathcal{D})$ such that the orders $\leq_{Q}$ and $\leq_{P}$ on $\mathcal{I}$ are the same. We have the filtrations $\mathcal{F}_{i}^{Q}(i=1,2)$ of $\mathfrak{V}_{\mid Q}$ corresponding to the meromorphic prolongments $\mathcal{Q E}(* W)$ and $\overline{\mathrm{HS}}(V)_{0}(* W)$. Because $\operatorname{HS}(V, \nabla)_{0} \simeq\left(\mathcal{E}, \widetilde{\mathbb{D}}^{f}\right)$, we have $\mathcal{F}_{1}^{Q}=\mathcal{F}_{2}^{Q}$. 
Let us show that $\mathcal{F}_{i}^{P}$ is obtained as the parallel transport of $\mathcal{F}_{i}^{Q}$, which implies $\mathcal{F}_{1}^{P}=\mathcal{F}_{2}^{P}$. We take $S_{Q} \in \mathcal{M S}\left(Q, \mathcal{X}-W, \phi_{0}^{*} \mathcal{I}\right)$ such that the Stokes filtrations $\mathcal{F}_{1}^{S_{Q}}$ of $\mathcal{Q} \mathcal{E}_{\mid \bar{S}_{Q}}$ and $\mathcal{F}_{2}^{S_{Q}}$ of $\phi_{0}^{*}(H)_{\mid \bar{S}_{Q}}$ exist. By using the characterization in Proposition 5.6. we obtain $\left(\mathcal{F}_{i}^{S_{P}}\right)_{\mid \bar{S}_{Q}}=\mathcal{F}_{i}^{S_{Q}}$. Hence, we can conclude that $\mathcal{F}_{i}^{P}$ are obtained as the parallel transport of $\mathcal{F}_{i}^{Q}$.

Lemma 8.12. The isomorphism $\mathcal{E} \simeq \operatorname{HS}(V)_{0}$ on $\mathcal{X}-\mathcal{D}$ extends to an isomorphism $\mathcal{Q} \mathcal{E} \overline{\mathrm{HS}}(V)_{0}$ on $\mathcal{X}$.

Proof. Let $P \in \pi^{-1}(0,0)$. We take a small multi-sector $S_{P} \in \mathcal{M S}(P, \mathcal{X}-W, \mathcal{I})$ such that we have the Stokes filtrations $\mathcal{F}^{S_{P}}$ for $\mathcal{Q}_{0} \mathcal{E}_{\mid \bar{S}_{P}}$ and $\phi_{0}^{*}(H)_{\mid \bar{S}_{P}}$. By Lemma 8.11 their restrictions to $S_{P}$ are the same. We take a flat splitting $\mathcal{E}_{\mid S_{P}}=\bigoplus_{\mathfrak{a} \in \mathcal{I}} \mathcal{E}_{\mathfrak{a}, S_{P}}$, which extends to the decompositions

$$
\mathcal{Q} \mathcal{E}_{\mid \bar{S}_{P}}=\bigoplus_{\mathfrak{a} \in \mathcal{I}} \mathcal{Q} \mathcal{E}_{\mathfrak{a}, S_{P}}, \quad \phi_{0}^{*}(H)_{\mid \bar{S}_{P}}=\bigoplus_{\mathfrak{a} \in \mathcal{I}} \phi_{0}^{*}(H)_{\mathfrak{a}, S_{P}}
$$

Let $\widetilde{\mathcal{L}}(-\mathfrak{a})$ be a line bundle $\mathcal{O}_{\mathcal{X}}(* W) \cdot e$ with $\nabla e=e \cdot\left(-d\left(\lambda^{-1} \mathfrak{a}(z)\right)\right)$. Because $\operatorname{Gr}_{\mathfrak{a}}\left(\phi_{0}^{*} H\right) \otimes \widetilde{\mathcal{L}}(-\mathfrak{a})$ and $\operatorname{Gr}_{\mathfrak{a}}(\mathcal{Q} \mathcal{E}) \otimes \widetilde{\mathcal{L}}(-\mathfrak{a})$ have regular singularity along $W$, the isomorphism on $\mathcal{X}-W$ naturally extends to an isomorphism $\operatorname{Gr}_{\mathfrak{a}}\left(\phi_{0}^{*} H\right) \otimes \widetilde{\mathcal{L}}(-\mathfrak{a}) \simeq$ $\operatorname{Gr}_{\mathfrak{a}}(\mathcal{Q E}) \otimes \widetilde{\mathcal{L}}(-\mathfrak{a})$. Since the restrictions of $\operatorname{Gr}_{\mathfrak{a}}\left(\phi_{0}^{*} H\right) \otimes \mathcal{O}(* \mathcal{D})$ and $\operatorname{Gr}_{\mathfrak{a}}(\mathcal{Q E})$ to $\mathcal{X}-\mathcal{D}$ are naturally isomorphic, we obtain an isomorphism $\operatorname{Gr}_{\mathfrak{a}}\left(\phi_{0}^{*} H\right) \otimes \mathcal{O}(* \mathcal{D}) \simeq$ $\operatorname{Gr}_{\mathfrak{a}}(\mathcal{Q E})$.

Let $\boldsymbol{w}_{\mathfrak{a}}$ and $\boldsymbol{v}_{\mathfrak{a}}$ be frames of $\mathrm{Gr}_{\mathfrak{a}}\left(\phi_{0}^{*} H\right) \otimes \mathcal{O}(* \mathcal{D})$ and $\operatorname{Gr}_{\mathfrak{a}}(\mathcal{Q E})$, respectively. We have the relation $\boldsymbol{w}_{\mathfrak{a}}=\boldsymbol{v}_{\mathfrak{a}} \cdot A_{\mathfrak{a}}$, where $A_{\mathfrak{a}}$ are meromorphic along $\mathcal{D}$. We take lifts $\boldsymbol{w}_{\mathfrak{a}, S}$ and $\boldsymbol{v}_{\mathfrak{a}, S}$ to $\mathcal{E}_{\mathfrak{a}, S}$ by using the above splittings for any small sector in $\mathcal{X}-W$. We have the relation $\boldsymbol{w}_{\mathfrak{a}, S}=\boldsymbol{v}_{\mathfrak{a}, S} \cdot A_{\mathfrak{a}}$. Then the isomorphism $\mathcal{E} \simeq \operatorname{HS}(V)_{0}$ extends to $\mathcal{Q} \mathcal{E}_{\mid \mathcal{U}} \simeq \overline{\mathrm{HS}}(V)_{0 \mid \mathcal{U}}$ on some small neighbourhood $\mathcal{U}$ of $(0,0)$, which follows from Proposition 5.5. (We may apply Lemma 5.6. But, since $\phi_{0}^{*} V_{0}$ and $\mathcal{Q}_{0} \mathcal{E}$ may not be good lattices, we replace them with an unramifiedly good lattice, or we use a variant of Lemma 5.6 for a pseudo-good lattice.) Then it is easy to observe that the isomorphism extends to $\mathcal{Q} \mathcal{E} \simeq \overline{\mathrm{HS}}(V)_{0}$ by using the Hartogs theorem. (Sabbah also independently obtained an argument to extend such isomorphisms in this kind of situation.)

Similarly, $\mathcal{E}^{\dagger} \simeq \operatorname{HS}(V)_{\infty}$ on $\mathcal{X}^{\dagger}-\mathcal{D}^{\dagger}$ extends to an isomorphism $\mathcal{Q} \mathcal{E}^{\dagger} \simeq$ $\overline{\mathrm{HS}}(V)_{\infty}$ on $\mathcal{X}^{\dagger}$.

Proof of the first claim. Let $\widetilde{\mathcal{X}}^{\triangle}$ denote the real blow up of $\mathbb{P}^{1} \times X$ along $\left(\mathbb{P}^{1} \times D\right) \cup(\{0\} \times X) \cup(\{\infty\} \times X)$. Let $\pi^{\dagger}: \widetilde{\mathcal{X}}^{\dagger}\left(W^{\dagger}\right) \rightarrow \mathcal{X}^{\dagger}$ denote the real blow 
up of $\mathbb{C}_{\mu} \times X^{\dagger}$ along $W^{\dagger}=\mathcal{D}^{\dagger} \cup\left(\{0\} \times X^{\dagger}\right)$. We have

$$
\widetilde{\mathcal{X}}^{\triangle}=\widetilde{\mathcal{X}}(W) \cup \widetilde{\mathcal{X}}^{\dagger}\left(W^{\dagger}\right) .
$$

Let $\mathfrak{V}^{\triangle}$ denote the flat bundle on $\tilde{\mathcal{X}}^{\triangleleft}$ associated to $\left(\mathcal{E}, \widetilde{\mathbb{D}}^{f}\right)_{\mid \mathbb{C}_{\lambda}^{*} \times(X-D)}$.

We have the $C^{\infty}$-map $\mathcal{X}-W \rightarrow\left(\mathbb{R}_{\geq 0} \times S^{1}\right)^{2}$ given by

$$
(\lambda, z) \mapsto((|\lambda|, \lambda /|\lambda|),(|z|, z /|z|))
$$

It induces the natural identification $\widetilde{\mathcal{X}}(W) \simeq\left(\mathbb{R}_{\geq 0} \times S^{1}\right)^{2}$. We set

$$
P_{0}=((0, \exp (\sqrt{-1} \varphi)),(1,1)) \in \pi^{-1}((0,1)) \subset \widetilde{\mathcal{X}}(W) .
$$

Similarly, we identify $\widetilde{\mathcal{X}}^{\dagger}\left(W^{\dagger}\right)$ with $\left(\mathbb{R}_{\geq 0} \times S^{1}\right)^{2}$ via the map induced by

$$
(\mu, z) \mapsto((|\mu|, \mu /|\mu|),(|z|, z /|z|)) .
$$

We set $Q_{0}:=((0, \exp (-\sqrt{-1} \varphi)),(1,1)) \in\left(\pi^{\dagger}\right)^{-1}((0,1)) \subset \widetilde{\mathcal{X}}^{\dagger}\left(W^{\dagger}\right)$. Note that we can identify $(V, \nabla)$ with $\operatorname{HS}(V, \nabla)_{\mid \mathbb{P}^{1} \times\{1\}}$. Hence, we have only to compare the Stokes filtrations $\mathcal{F}^{P_{0}}\left(\mathfrak{V}_{\mid P_{0}}^{\triangleleft}\right)$ and $\mathcal{F}^{Q_{0}}\left(\mathfrak{V}_{\mid Q_{0}}^{\triangleleft}\right)$ under the parallel transport along the ray $((s, \exp (\sqrt{-1} \varphi)),(1,1))\left(s \in \mathbb{R}_{\geq 0} \cup\{+\infty\}\right)$ connecting $P_{0}$ and $Q_{0}$. (Note that the signature of the arguments are reversed by the coordinate change $\lambda^{-1}=\mu$.)

Let us consider the map $G:[0,1] \times[0,1] \rightarrow \widetilde{\mathcal{X}}(W)$ given by

$$
G(s, t)=((s, \exp (\sqrt{-1} \varphi)),(t, 1)) .
$$

Note $G(0,1)=P_{0}$. We set $P_{1}:=G(1,0)$ and $P_{2}:=G(1,1)$. The image of $\Gamma_{0}:=$ $([0,1] \times\{0\}) \cup(\{0\} \times[0,1])$ is contained in $\pi^{-1}(W)$. The orders $\leq_{P}$ are independent of $P \in G\left(\Gamma_{0}\right)$. Hence, the Stokes filtrations are unchanged along $G\left(\Gamma_{0}\right)$.

Similarly, let us consider the map $G^{\dagger}:[0,1] \times[0,1] \rightarrow \widetilde{\mathcal{X}}^{\dagger}\left(W^{\dagger}\right)$ given by

$$
G^{\dagger}(s, t)=((s, \exp (-\sqrt{-1} \varphi)),(t, 1)) .
$$

Note $G^{\dagger}(0,1)=Q_{0}$. We set $Q_{1}:=G^{\dagger}(1,0)$ and $Q_{2}:=G^{\dagger}(1,1)$. The image of $\Gamma_{\infty}:=([0,1] \times\{0\}) \cup(\{0\} \times[0,1])$ is contained in $\left(\pi^{\dagger}\right)^{-1}\left(W^{\dagger}\right)$. The orders $\leq_{Q}$ are independent of the choice of $Q \in G^{\dagger}\left(\Gamma_{\infty}\right)$. Hence, the Stokes filtrations are unchanged along $G^{\dagger}\left(\Gamma_{\infty}\right)$.

Under the identification $\mathcal{X}-W=\mathcal{X}^{\dagger}-W^{\dagger}$, we have $P_{2}=Q_{2}$, and the union of the paths $G([0,1] \times\{1\})$ and $G^{\dagger}([0,1] \times\{1\})$ is the ray connecting $P_{0}$ and $Q_{0}$. Hence, for the comparison of $\mathcal{F}^{P_{0}}$ and $\mathcal{F}^{Q_{0}}$, we have only to show that $\mathcal{F}^{P_{1}}\left(\mathfrak{V}_{0 \mid P_{1}}\right)$ of $\mathfrak{V}_{\mid P_{1}}$ and $\mathcal{F}^{Q_{1}}\left(\mathfrak{V}_{\infty \mid Q_{1}}\right)$ of $\mathfrak{V}_{\mid Q_{1}}$ are the same. This follows from the characterization of the Stokes filtrations of $(\mathcal{Q E}, \mathbb{D})$ and $\left(\mathcal{Q E}^{\dagger}, \mathbb{D}^{\dagger}\right)$ by growth order of the norms of flat sections with respect to the metric $h$. (See Subsection 6.3) Thus, we obtain the first claim of Theorem 8.1. 
Proof of the second claim. By using Corollary 5.1 and Lemma 8.8, we obtain the isomorphisms on $\mathbb{P}^{1} \times X^{*}(R)$ for some $R>0$ :

$$
\operatorname{Gr}_{\mathfrak{a}}\left(\mathcal{E}^{\triangle}, \widetilde{\mathbb{D}}^{\triangle}, \mathcal{S}_{E}\right) \simeq \operatorname{Gr}_{\psi_{0}^{*} \mathfrak{a}} \operatorname{HS}(V, \nabla, \mathcal{S}) \simeq \operatorname{HSGr}_{\mathfrak{a}}(V, \nabla, \mathcal{S})
$$

Thus, the second claim is also proved.

\section{Acknowledgements}

This paper grew out of my effort to understand the work due to Claus Hertling, Claude Sabbah and Christian Sevenheck on TERP structures and Integrable twistor structures. I am grateful to them for attracting my attention to this subject. I also thank them for their comments on the earlier versions of this paper. In particular, Hertling kindly sent a surprisingly detailed and careful letter [11, which was quite helpful for improving this paper and correcting some errors in earlier versions.

I wish to express my thanks to Yoshifumi Tsuchimoto and Akira Ishii for their constant encouragement.

I gave talks on the sequence (1.1) at the conferences "From tQFT to $\mathrm{tt}^{\text {* }}$ and Integrability" in Augsburg and "New Developments in Algebraic Geometry, Integrable Systems and Mirror Symmetry" in Kyoto. This paper is an enhancement of the talks. It is my great pleasure to express my gratitude to the organizers of the conferences, Ron Donagi, Katrin Wendland, Masa-Hiko Saito, and Kōta Yoshioka, on this occasion.

I heartily thank the referee for his/her valuable comments.

I am grateful to the partial financial support by Ministry of Education, Culture, Sports, Science and Technology.

\section{References}

[1] C. Banica, Le complété formel d'un espace analytique le long d'un sous-espace: Un théoréme de comparaison, Manuscripta Math. 6 (1972), 207-244. Zbl 0231.32004 MR 0301231

[2] J. Bingener, Über formale komplexe Räume, Manuscripta Math. 24 (1978), 253-293. Zbl 0381.32015 MR 0492367

[3] O. Biquard and P. Boalch, Wild non-abelian Hodge theory on curves, Compos. Math. 140 (2004), 179-204. Zbl 1051.53019 MR 2004129

[4] N. Borne, Fibrés paraboliques et champ des racines, Int. Math. Res. Notices 2007, no. 16, 38 pp. Zbl 1197.14035 MR 2353089

[5] _ Sur les représentations du groupe fondamental d'une variété privée d'un diviseur à croisements normaux simples, Indiana Univ. Math. J. 58 (2009), 137-180. Zbl 1186.14016 MR 2504408 
[6] E. Cattani and A. Kaplan, Polarized mixed Hodge structures and the local monodromy of variation of Hodge structure, Invent. Math. 67 (1982), 101-115. Zbl 0516.14005 MR 0664326

[7] E. Cattani, A. Kaplan and W. Schmid, Degeneration of Hodge structures, Ann. of Math. 123 (1986), 457-535. Zbl 0617.14005 MR 0840721

[8],$L^{2}$ and intersection cohomologies for a polarized variation of Hodge structure, Invent. Math. 87 (1987), 217-252. Zbl 0611.14006 MR 0870728

[9] P. Deligne, Équations différentielles à points singuliers réguliers, Lecture Notes in Math. 163, Springer, 1970. Zbl 0244.14004 MR 0417174

[10] C. Hertling, $t t^{*}$ geometry, Frobenius manifolds, their connections, and the construction for singularities, J. Reine Angew. Math. 555 (2003), 77-161. Zbl 1040.53095 MR 1956595

[11] , letter to the author, 30 Sep. 2008.

[12] C. Hertling and C. Sevenheck, Nilpotent orbits of a generalization of Hodge structures, J. Reine Angew. Math. 609 (2007), 23-80. Zbl 1136.32011 MR 2350780

[13] C. Hertling and C. Sevenheck, Limits of families of Brieskorn lattices and compactified classifying spaces, Adv. Math. 223 (2010), 1155-1224. Zbl 1190.14011|| MR 2581368

[14] C. Hertling and C. Sevenheck, Twistor structures, tt*-geometry and singularity theory, in From Hodge theory to integrability and TQFT tt*-geometry, Proc. Sympos. Pure Math. 78, Amer. Math. Soc., Providence, RI, 2008, 49-73. Zbl 1151.14304 MR 2483748

[15] J. Iyer and C. Simpson, A relation between the parabolic Chern characters of the de Rham bundles, Math. Ann. 338 (2007), 347-383. Zbl 1132.14006 MR 2302066

[16] M. Kashiwara, The asymptotic behavior of a variation of polarized Hodge structure, Publ. RIMS Kyoto Univ. 21 (1985), 853-875. Zbl 0594.14012 MR 0817170

[17] M. Kashiwara and T. Kawai, The Poincaré lemma for variations of polarized Hodge structure, Publ. RIMS Kyoto Univ. 23 (1987), 345-407. Zbl 0629.14005 MR 0890924

[18] L. Katzarkov, M. Kontsevich and T. Pantev, Hodge theoretic aspects of mirror symmetry, in From Hodge theory to integrability and TQFT tt ${ }^{*}$-geometry, Proc. Sympos. Pure Math. 78, Amer. Math. Soc., Providence, RI, 2008, 87-174. Zbl 1206.14009 MR 2483750

[19] B. A. Krasnov, Formal modifications. Existence theorems for modifications of complex manifolds, Math. USSR Izv. 7 (1973), 847-881. Zbl 0291.32018 MR 0330504

[20] H. Majima, Asymptotic analysis for integrable connections with irregular singular points, Lecture Notes in Math. 1075, Springer, Berlin, 1984. Zbl 0546.58003 MR 0757897

[21] T. Mochizuki, Asymptotic behaviour of tame nilpotent harmonic bundles with trivial parabolic structure, J. Differential Geom. 62 (2002), 351-559. Zbl 1069.32010 MR 2005295

[22] - Asymptotic behaviour of tame harmonic bundles and an application to pure twistor D-modules, I, II, Mem. Amer. Math. Soc. 185 (2007), nos. 869, 870. Zbl pre05119702 (I), Zbl pre05119703 (II) MR 2281877(I), MR 2283665 (II)

[23] Wild harmonic bundles and wild pure twistor $D$-modules, Astérisque, to appear; arXiv:0803.1344, ver. 3.

[24] C. Sabbah, Équations différentielles à points singuliers irréguliers et phénomène de Stokes en dimension 2, Astérisque 263 (2000). Zbl 0947.32005 MR 1741802

[25] _ Polarizable twistor D-modules, Astérisque 300 (2005). Zbl 1085.32014 MR 2156523

[26] - Fourier-Laplace transform of a variation of polarized complex Hodge structure, II, in New developments in algebraic geometry, integrable systems and mirror symmetry (Kyoto, 2008), Adv. Stud. Pure Math. 59, Math. Soc. Japan, Tokyo, 2010, 289-347. MR 2683213 
[27] K. Saito and A. Takahashi, From primitive forms to Frobenius manifolds, in From Hodge theory to integrability and TQFT tt* -geometry, Proc. Sympos. Pure Math. 78, Amer. Math. Soc., Providence, RI, 2008, 31-48. Zbl 1161.32013 MR 2483747

[28] W. Schmid, Variation of Hodge structure: the singularities of the period mapping, Invent. Math. 22 (1973), 211-319. Zbl 0278.14003 MR 0382272

[29] Y. Sibuya, Perturbation of linear ordinary differential equations at irregular singular points, Funkcial. Ekvac. 11 (1969), 235-246. Zbl 0228.34036 MR 0243171

[30] C. Simpson, Harmonic bundles on non-compact curves, J. Amer. Math. Soc. 3 (1990), 713770. Zbl 0713.58012 MR 1040197

[31] _ Higgs bundles and local systems, Publ. I.H.E.S. 75 (1992), 5-95. Zbl 0814.32003 MR 1179076

[32] , Mixed twistor structures, arXiv:alg-geom/9705006.

[33] - The Hodge filtration on nonabelian cohomology, in Algebraic geometry-Santa Cruz 1995, Proc. Sympos. Pure Math. 62, Part 2, Amer. Math. Soc., Providence, RI, 1997, 217-281. Zbl 0914.14003 MR 1492538

[34] W. Wasow, Asymptotic expansions for ordinary differential equations, reprint of 1976 ed., Dover Publ., New York, 1987. Zbl 0644.34003 MR 0919406 